\title{
Experimental and Numerical Investigation of Centrifugal Compressors for use with Alternative Working Fluids
}

\author{
by \\ Steven Elia \\ B.Eng. (Aerospace) \\ A thesis submitted to \\ the Faculty of Graduate Studies and Research \\ in partial fulfillment of the requirements \\ for the degree of

\section{Master of Applied Science} \\ in \\ Aerospace Engineering \\ Ottawa-Carleton Institute for Mechanical \\ and Aerospace Engineering \\ Department Of Mechanical and Aerospace Engineering \\ Carleton University \\ Ottawa, Ontario, Canada \\ March 10th, 2008 \\ (C) 2008 \\ Steven Elia
}




$\begin{array}{ll}\begin{array}{l}\text { Library and } \\ \text { Archives Canada }\end{array} & \begin{array}{l}\text { Bibliothèque et } \\ \text { Archives Canada }\end{array} \\ \begin{array}{l}\text { Published Heritage } \\ \text { Branch }\end{array} & \begin{array}{l}\text { Direction du } \\ \text { Patrimoine de l'édition }\end{array} \\ \begin{array}{l}\text { 395 Wellington Street } \\ \text { Ottawa ON K1A 0N4 } \\ \text { Canada }\end{array} & \begin{array}{l}\text { 395, rue Wellington } \\ \text { Ottawa ON K1A 0N4 } \\ \text { Canada }\end{array}\end{array}$

Your file Votre référence ISBN: 978-0-494-40637-3

Ourfile Notre référence

ISBN: 978-0-494-40637-3

NOTICE:

The author has granted a nonexclusive license allowing Library and Archives Canada to reproduce, publish, archive, preserve, conserve, communicate to the public by telecommunication or on the Internet, loan, distribute and sell theses worldwide, for commercial or noncommercial purposes, in microform, paper, electronic and/or any other formats.

The author retains copyright ownership and moral rights in this thesis. Neither the thesis nor substantial extracts from it may be printed or otherwise reproduced without the author's permission.
AVIS:

L'auteur a accordé une licence non exclusive permettant à la Bibliothèque et Archives Canada de reproduire, publier, archiver, sauvegarder, conserver, transmettre au public par télécommunication ou par l'Internet, prêter, distribuer et vendre des thèses partout dans le monde, à des fins commerciales ou autres, sur support microforme, papier, électronique et/ou autres formats.

L'auteur conserve la propriété du droit d'auteur et des droits moraux qui protège cette thèse. $\mathrm{Ni}$ la thèse ni des extraits substantiels de celle-ci ne doivent être imprimés ou autrement reproduits sans son autorisation.
In compliance with the Canadian Privacy Act some supporting forms may have been removed from this thesis.

While these forms may be included in the document page count, their removal does not represent any loss of content from the thesis.
Conformément à la loi canadienne sur la protection de la vie privée, quelques formulaires secondaires ont été enlevés de cette thèse.

Bien que ces formulaires aient inclus dans la pagination, il n'y aura aucun contenu manquant.

\section{Canada}




\section{ABSTRACT}

Investigations into a zero-emission gas turbine engine having carbon dioxide as the working fluid have raised a number of questions regarding the effects of working fluid properties on the aerodynamics of turbomachinery. The present research investigated the aerodynamic performance of centrifugal compressors for use in alternative working fluids. The research focused on the re-design and analysis of a new centrifugal compressor which uses carbon dioxide as the working fluid. It was intended to provide both experimental data and computational predictions for the compressor performance. However, due to time constraints only detailed aerodynamic predictions could be made. Instead, experimental data were collected for a commercial centrifugal compressor from an automotive turbocharger. The experimental data from the turbocharger compressor were used to validate the computational methods for the same geometry. In addition, the experimental testing was used to develop new measurement techniques which provide measurements of the impeller outlet flow.

The re-designed compressor was also used to investigate the influence of working fluid on the compressor design geometry. To do so, a process using simple geometric modifications was developed to counteract the effect of the isentropic exponent on the impeller performance. It was found that the change in performance resulting from the change in working fluid could be partially counteracted.

Comparisons between the computations and the measurements made just downstream of the turbocharger compressor rotor demonstrated the challenges involved in obtaining impeller-only performance measurements. These challenges were the result of time varying and highly non-uniform flow exiting the rotor. The detailed aerodynamic predictions of the re-designed centrifugal compressor showed significant spanwise and circumferential variations in the impeller flow. 


\section{ACKNOWLEDGEMENTS}

I would like to thank my supervisor, Professor S. Sjolander for his guidance and support to make this thesis work possible. I am also greatly appreciative of the knowledge and expertise in the gas turbine industry that he has shared with me over the past few years. I would also like to thank the staff of the Department of Mechanical and Aerospace Engineering. In particular, a special thanks to Alex Proctor, Kevin Sangster, Jim Sliwka and Steve Truttmann for their help and expertise in the machine shop and other technical matters. Also, thanks to Fred Barrett and Neil McFadyen for their help with the electronics and computers needed to perform this study. Finally, I would like to thank my colleagues, friends and family for their support and patience during the course of my work. 


\section{TABLE OF CONTENTS}

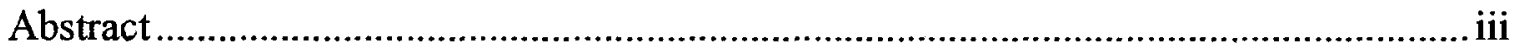

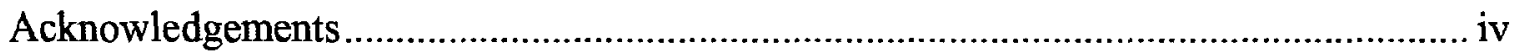

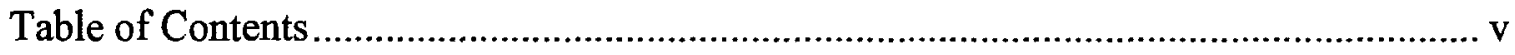

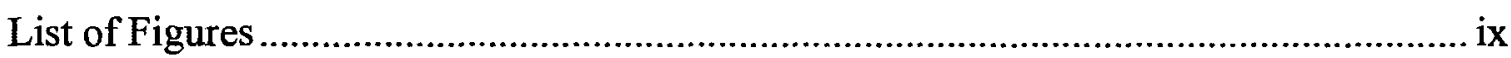

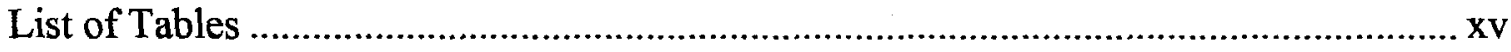

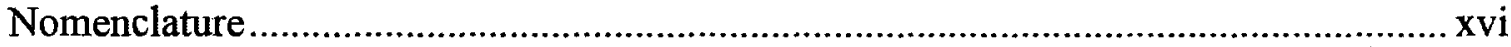

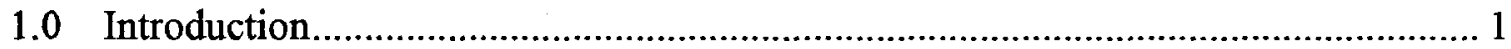

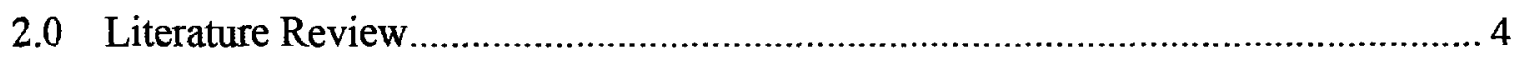

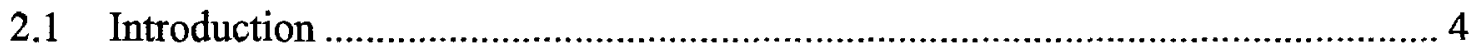

2.2 Centrifugal Compressor Design ................................................................. 4

2.3 Alternative Working Fluids For Gas Turbine Cycles........................................ 16

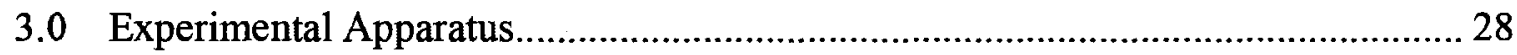

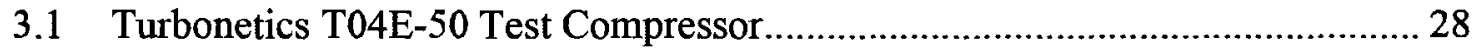

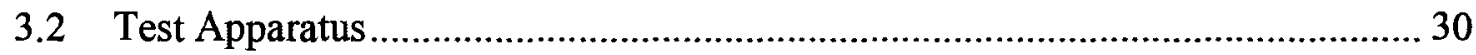

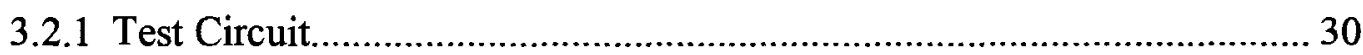

3.2.2 High Speed Wind Tunnel Facility and Control System............................ 32

4.0 Methods of Measurement and Data Reduction....................................................... 35

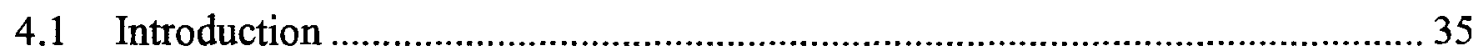

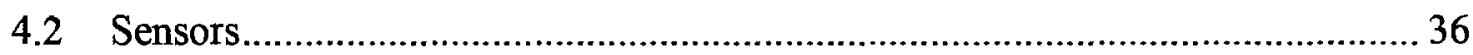

4.2.1 Differential Pressure Transducer ............................................................ 36

4.2.2 Absolute Pressure Transducer and Scanivalve $($....................................... 37

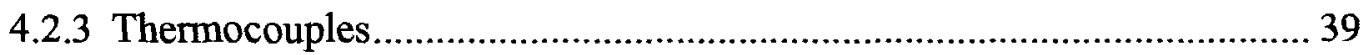

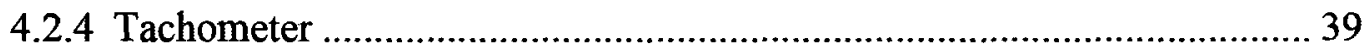

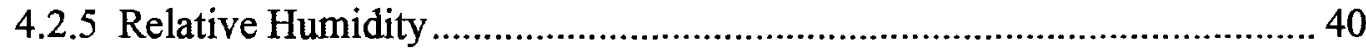

4.3 Probes Previously Installed by Roberts ........................................................ 40

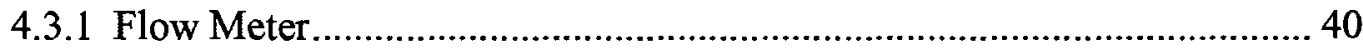

4.3.2 Inlet Pressure Probes................................................................................ 41

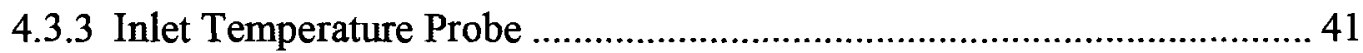


4.3.4 Discharge Temperature/Pressure Probe ...................................................... 42

4.4 Probes Added During Test Rig Modifications .................................................... 43

4.4.1 Impeller Outlet Total Pressure Probes ........................................................ 43

4.4.2 Shroud Surface Static Pressure Taps ....................................................... 45

4.4.3 Volute Static Pressure Taps .................................................................. 47

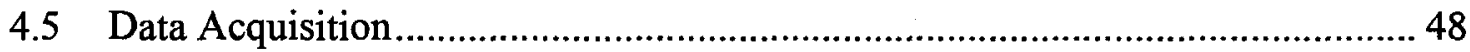

4.5.1 Summary of the Data Acquisition System................................................. 48

4.5.2 Pressure and Temperature Measurements ............................................... 49

4.5.3 Shaft Speed Measurement...................................................................... 51

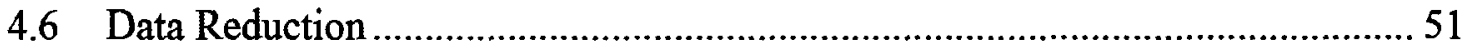

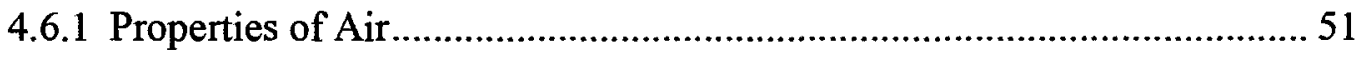

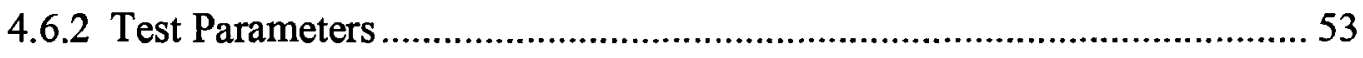

4.6.3 Corrections to Measured Data ............................................................... 59

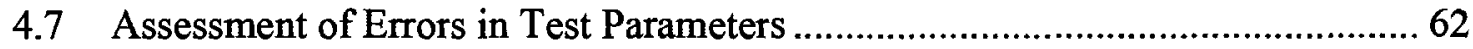

4.7.1 Uncertainties in Measured Test Quantities ............................................... 62

4.7.2 Uncertainties in Calculated Test Parameters ............................................. 66

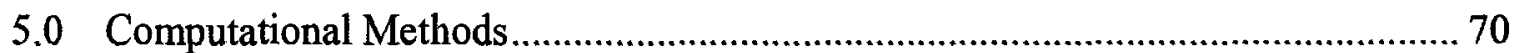

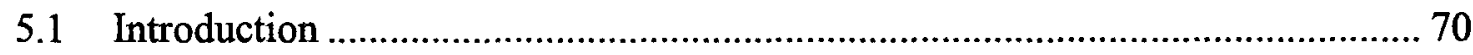

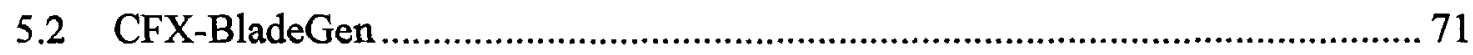

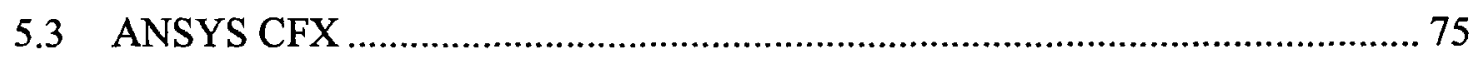

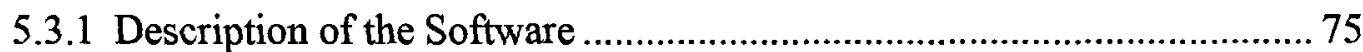

5.3.2 Modelling of the Turbonetics T04E-50 Impeller..................................... 76

5.3.3 Modelling of the Test Rig Compressor Impeller ....................................... 82

5.3.4 Modelling of the Test Rig Compressor Stage............................................. 83

6.0 Influence of Working Fluid on Compressor Design Geometry ............................... 86

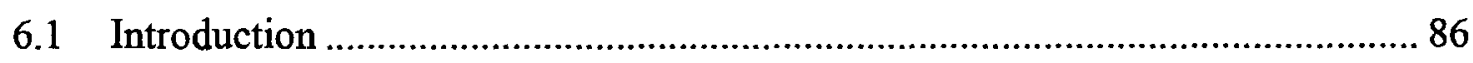

6.2 Design of the Rotating Test Rig Impeller...................................................... 87

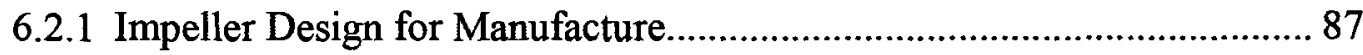

6.2.2 Detailed Aerodynamic Performance of Test Rig Impeller ...................... 100 
6.3 Effect of Fluid Properties on Impeller Outlet Geometry .................................. 107

7.0 Turbonetics T04E-50 Compressor Results and Discussion................................... 123

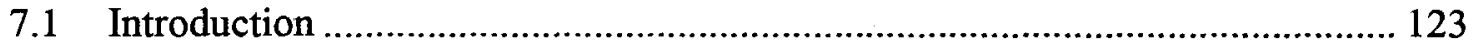

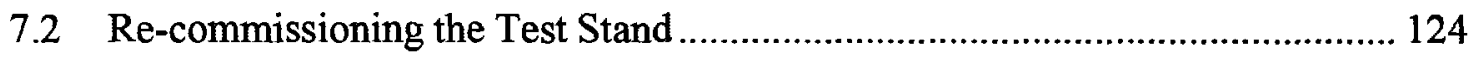

7.3 Experimental Performance Results ................................................................. 129

7.3.1 Effects of Rig Modifications on Stage Performance Characteristics...... 129

7.3.2 Impeller versus Stage Performance Characteristics.............................. 132

7.3.3 Impeller Circumferential Variations Measured by Rotatable Total Pressure

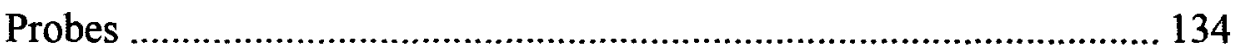

7.3.4 Static Pressure Variation on Shroud Surface ......................................... 137

7.3.5 Volute Static Pressure Variation........................................................... 139

7.4 T04E-50 Impeller Performance Predictions Using ANSYS CFX .................... 141

7.4.1 Impeller Performance Characteristics ..................................................... 141

7.4.2 Exit Flow Angle Predictions for Varying Mass Flow Rates.................... 146

7.4.3 Downstream Total Pressure and Flow Angle Distributions ................... 149

7.4.4 Shroud Surface Static Pressures............................................................. 152

8.0 Test Rig Compressor Performance Predictions Using ANSYS CFX ................... 155

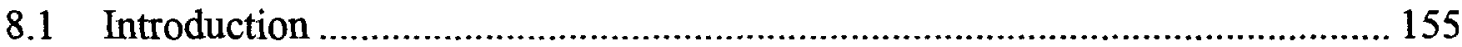

8.2 Test Rig Impeller Performance Predictions ................................................ 156

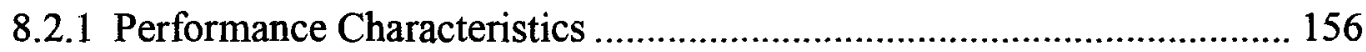

8.2.2 Detailed Aerodynamic Performance...................................................... 158

8.2.3 Effect of Tip Clearance on Impeller Performance ................................. 163

8.3 Stage Performance Predictions ...................................................................... 166

8.3.1 Stage Performance Characteristics ...................................................... 166

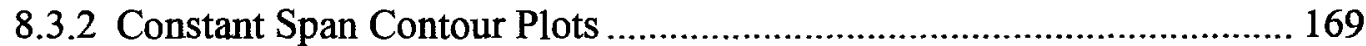

8.3.3 Exit Plane Total Pressure and Flow Angle Distributions ……................ 176

8.3.4 Blade Loadings ........................................................................... 180

8.4 Volute Performance and Its Effect on the Impeller.......................................... 182

8.4.1 Performance Characteristics With and Without Volute........................... 182 
8.4.2 Mach Number and Total Pressure Contours......................................... 185

9.0 Conclusions and Recommendations for Future Work …................................... 190

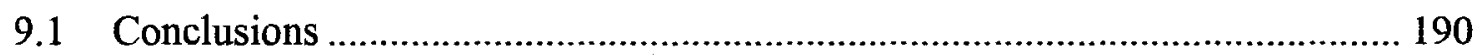

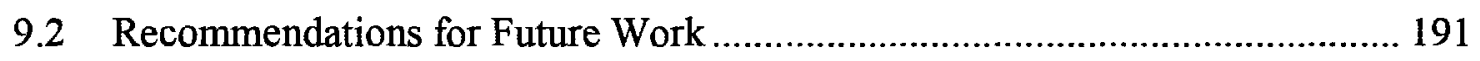

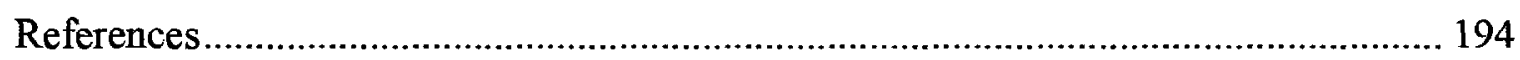

Appendix: Curve Fit Equations of Fluid Properties ……............................................ 199

viii 


\section{LIST OF FIGURES}

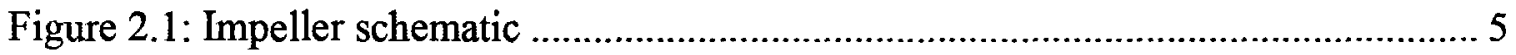

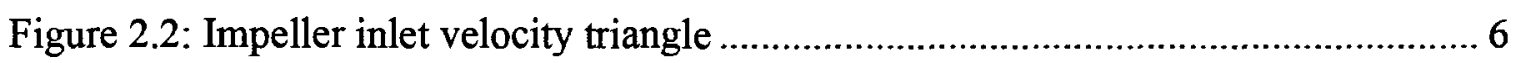

Figure 2.3: Impeller outlet velocity triangle .............................................................. 10

Figure 2.4: Effect of exit vane height on outlet velocity triangle ................................... 10

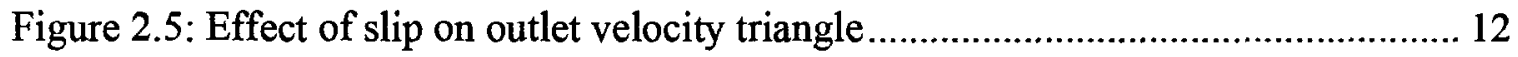

Figure 2.6: Schematic representation of a closed-cycle gas turbine for power generation

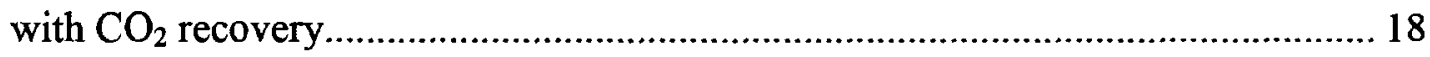

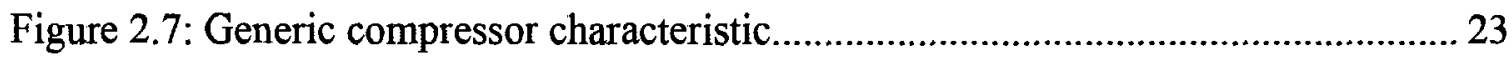

Figure 3.1: Schematic of the T04E-50 impeller (Axial cross-section) ........................... 29

Figure 3.2: Rotating test rig circuit schematic ........................................................... 31

Figure 3.3: Fully assembled test rig in closed-cycle configurations (Roberts, 2001)...... 32

Figure 3.4: High Speed Wind Tunnel Facility (Jeffries, 1994) ..................................... 33

Figure 3.5: Control system flow chart ..................................................................... 34

Figure 4.1: Differential pressure transducer calibration curve …….............................. 37

Figure 4.2: Absolute pressure transducer calibration ..................................................... 38

Figure 4.3: Inlet total temperature probe tip ............................................................ 41

Figure 4.4: Discharge total temperature/total pressure probe tip.................................... 42

Figure 4.5: Schematic of an assembled impeller outlet total pressure probe.................... 43

Figure 4.6: Impeller outlet total pressure probe geometry............................................. 44

Figure 4.7: Schematic of the impeller outlet total pressure probe locations..................... 45

Figure 4.8: Schematic showing the shroud surface static tap locations........................... 46

Figure 4.9: Schematic showing the volute static pressure tap locations .......................... 47

Figure 4.10: Signal flow chart of the instrumentation and data acquisition system ......... 48

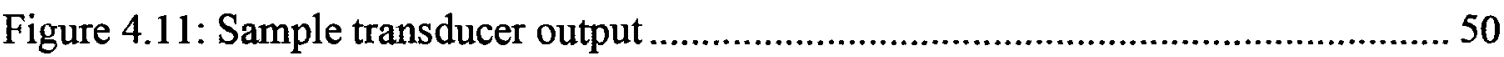

Figure 4.12: Sample measurements and curve fits obtained from the rotatable total

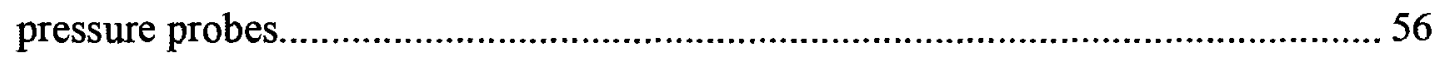

Figure 4.13: Averaged total pressure estimates from the sample measurements of the

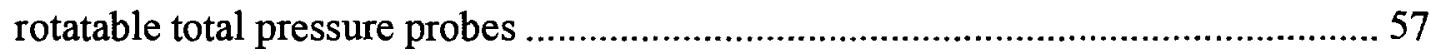


Figure 5.1: Charest (2003) impeller grid sensitivity in CFX-BladeGen+

Figure 5.2: Rotating test rig impeller grid sensitivity in CFX-BladeGen+ 74

Figure 5.3: Computational domains for the Turbonetics T04E-50 compressor impeller. 77

Figure 5.4: Slice plane of Turbonetics T04E-50 grid at constant axial location ............. 78

Figure 5.5: Turbonetics T04E50 grid sensitivity in ANSYS CFX ................................. 81

Figure 5.6: Computational domains of the test rig compressor stage in ANSYS CFX.... 84

Figure 6.1: Influence of blade count on impeller pressure ratio ...................................... 90

Figure 6.2: Influence of blade count on impeller slip factor......................................... 91

Figure 6.3: Influence of blade count on impeller efficiency......................................... 92

Figure 6.4: Influence of blade thickness on impeller pressure ratio ................................. 94

Figure 6.5: Influence of blade thickness on impeller efficiency.................................... 95

Figure 6.6: Influence of wrap angle on impeller efficiency........................................... 96

Figure 6.7: Rotating test rig impeller geometry and velocity triangles ........................... 98

Figure 6.8: Total pressure ratio characteristic of the test rig impeller predicted by CFX-

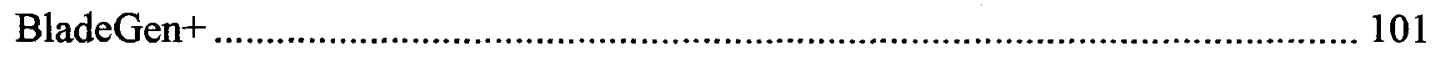

Figure 6.9: Isentropic efficiency characteristic of the test rig impeller predicted by CFX-

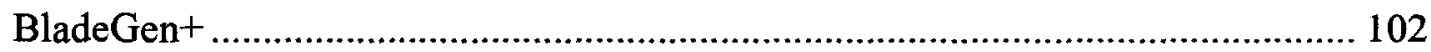

Figure 6.10: Contours of relative Mach number for the test rig impeller predicted by

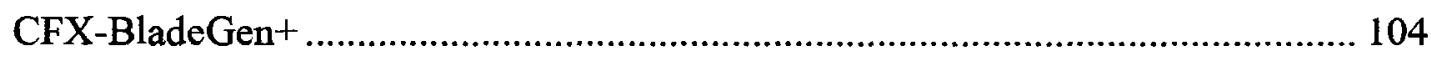

Figure 6.11: Contours of relative total pressure for the test rig impeller predicted by CFX-

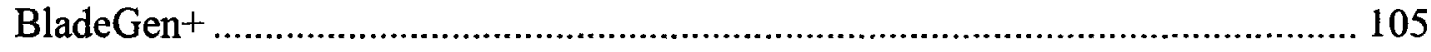

Figure 6.12: Contours of absolute total pressure for the test rig impeller predicted by

CFX-BladeGent 106

Figure 6.13: Blade loading distributions at mid-span for the test rig impeller predicted by

CFX-BladeGen+ 107

Figure 6.14: Total pressure ratio characteristic for air and $\mathrm{CO}_{2}$ predicted by CFXBladeGen + and Roberts (2001) correlations for the test rig impeller..................... 110

Figure 6.15: Efficiency characteristic for air and $\mathrm{CO}_{2}$ predicted by CFX-BladeGen+ and Roberts (2001) correlations for the test rig impeller 
Figure 6.16: Effect of exit vane height on rotor outlet velocity triangle

Figure 6.17: Effect of backsweep angle on rotor outlet velocity triangle.

Figure 6.18: Total pressure ratio characteristic of the modified impeller geometry

Figure 6.19: Efficiency characteristic of the modified impeller geometry.

Figure 7.1: Total pressure ratio characteristic of the T04E-50 compressor originally measured by Roberts (2001) 125

Figure 7.2: Efficiency characteristic of the T04E-50 compressor originally measured by Roberts (2001) 126

Figure 7.3: Measured total pressure ratio characteristic of the T04E-50 compressor compared to manufacturer's data. 127

Figure 7.4: Measured efficiency characteristic of the T04E-50 compressor compared to manufacturer's data. 128

Figure 7.5: Stage total pressure ratio characteristic of the T04E-50 compressor with and without new total pressure probes. 130

Figure 7.6: Effect of total pressure probes on the stage efficiency characteristic of the T04E-50 compressor

Figure 7.7: Measured impeller and stage total pressure ratio characteristic of the T04E-50 compressor.

Figure 7.8: Measured impeller and stage efficiency characteristic of the T04E-50 compressor. 134

Figure 7.9: Circumferential variation of total pressure measured at the T04E-50 impeller outlet 135

Figure 7.10: Circumferential variation of flow angle measured at the T04E-50 impeller outlet 136

Figure 7.11: Schematic of the shroud surface static tap locations 137

Figure 7.12: Static pressure variation measured on the shroud surface of the T04E-50 compressor 138

Figure 7.13: Schematic of the volute static tap locations 139 
Figure 7.14: Circumferenctial variation of static pressure variation measured on the volute of the T04E-50 compressor. 140

Figure 7.15: Total pressure ratio predicted by ANSYS CFX using outlet total pressure values mass-averaged over a thin ring, compared to measured data for the T04E-50 impeller 142

Figure 7.16: Efficiency predicted by ANSYS CFX using outlet total pressure values mass-averaged over a thin ring, compared to measured data for the T04E-50 impeller

Figure 7.17: Total pressure ratio predicted by ANSYS CFX using outlet total pressure values mass-averaged across the full flow area, compared to measured data for the T04E-50 impeller

Figure 7.18: Efficiency predicted by ANSYS CFX using outlet total pressure values mass-averaged across the full flow area, compared to measured data for the T04E-50 impeller 146

Figure 7.19: Exit flow angle predicted using ANSYS CFX mass-averaged across the thin ring area, compared to measured data for the T04E-50 impeller 147

Figure 7.20: Exit flow angle predicted using ANSYS CFX mass-averaged across the full flow area, compared to measured data for the T04E-50 impeller. 149

Figure 7.21: Contours of total pressure downstream of the T04E-50 impeller outlet .... 150

Figure 7.22: Contours of flow angle downstream of the T04E-50 impeller outlet......... 151

Figure 7.23: Shroud surface static pressure variation predicted by ANSYS CFX compared to measured data for the T04E-50 impeller.

Figure 7.24: Contours of the static pressure coefficient, $C_{P s}$ at multiple span locations for the T04E-50 impeller 154

Figure 8.1: Total pressure characteristic of the test rig impeller predicted by ANSYS CFX 157

Figure 8.2: Efficiency characteristic of the test rig impeller predicted by ANSYS CFX158 Figure 8.3: Contours of relative Mach number at multiple spans of the test rig impeller predicted by ANSYS CFX 159 
Figure 8.4: Contours of relative total pressure at multiple spans of the test rig impeller predicted by ANSYS CFX 161

Figure 8.5: Blade aerodynamic loading at mid span of the test rig impeller predicted by ANSYS CFX

Figure 8.6: Blade aerodynamic loading at multiple spans of the test rig impeller main blade predicted by ANSYS CFX 163

Figure 8.7: Effect of tip clearance on the predicted pressure rise characteristic of the test rig impeller. 165

Figure 8.8: Effect of tip clearance on the predicted efficiency characteristic of the test rig impeller 166

Figure 8.9: Total pressure ratio characteristic of the test rig impeller and stage predicted by ANSYS CFX. 168

Figure 8.10: Efficiency characteristic of the test rig impeller and stage predicted by ANSYS CFX

Figure 8.11: Contours of relative Mach number at multiple spans in the test rig impeller predicted by ANSYS CFX for the complete stage ...... 101

Figure 8.12: Contours of relative total pressure at multiple spans in the test rig impeller predicted by ANSYS CFX for the complete stage 101

Figure 8.13: Contours of static pressure at multiple spans in the test rig impeller predicted by ANSYS CFX for the complete stage 174

Figure 8.14: Contours of absolute total pressure at mid-span of the test rig impeller predicted by ANSYS CFX for the complete stage 176

Figure 8.15: Variation of total pressure between $0^{\circ}$ and $36^{\circ}$ circumference at the exit plane of the test rig impeller predicted in the complete stage. 177

Figure 8.16: Variation of total pressure between $180^{\circ}$ and $216^{\circ}$ circumference at the exit plane of the test rig impeller predicted in the complete stage. 178

Figure 8.17: Variation of flow angle between $0^{\circ}$ and $36^{\circ}$ circumference at the exit plane of the test rig impeller predicted in the complete stage 179 
Figure 8.18 Variation of flow angle between $180^{\circ}$ and $216^{\circ}$ circumference at the exit plane of the test rig impeller predicted in the complete stage. 180

Figure 8.19: Main blade aerodynamic loading distribution from the test rig compressor stage 167

Figure 8.20: Splitter blade aerodynamic loading distribution from the test rig compressor stage 167

Figure 8.21: Total pressure ratio characteristic of the test rig impeller with and without the volute predicted by ANSYS CFX 183

Figure 8.22: Efficiency characteristic of the test rig impeller with and without the volute predicted by ANSYS CFX 184

Figure 8.23: Contours of Mach number within the volute domain 186

Figure 8.24: Mass flow rate distribution at the volute inlet.......................................... 187

Figure 8.25: Mach number distribution near the volute exit ....................................... 188

Figure 8.26: Contours of total pressure within the volute domain ................................. 189

Figure 8.27: Total pressure distribution near the volute exit...................................... 189 


\section{LIST OF TABLES}

Table 2.1: Suggested de Haller number limits (from Sjolander, 2008) ........................... 11

Table 3.1: Summary of T04E-50 impeller geometry .................................................. 29

Table 4.1: Summary of the shroud surface static tap coordinates ................................... 46

Table 4.2: Typical scatter in single test point measurements ............................................6 63

Table 4.3: Estimated uncertainty in calculated test parameters..................................... 68

Table 5.1: Grid properties and run time for various grid refinement factors in CFX-

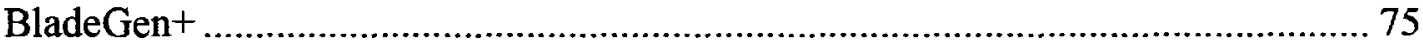

Table 6.1: Summary of performance parameters for the preliminary impeller designed by

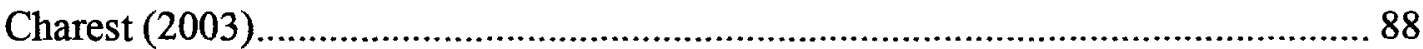

Table 6.2: Summary of original (Charest, 2003) and rotating test rig impeller geometries 99

Table 6.3: Summary of original (Charest, 2003) and rotating test rig impeller performance parameters 100

Table 6.4: Summary of performance parameters for air and $\mathrm{CO}_{2}$ as predicted by BladeGen + at the reference operating points of the test rig impeller 113

Table 6.5: Expected effect of geometric parameters on impeller performance 117

Table 6.6: CFX-BladeGen+ performance predictions for various geometric modifications 119

Table 6.7: Geometric modifications required to counteract the change in performance due to the change in working fluid 120 


\section{NOMENCLATURE}

\section{Symbols:}

Units

A

$a$

B

b

C

$C_{D}$

$C_{p s}$

$c_{p}$

$\mathcal{c}_{v}$

D

$D_{H}$

$e$

$L$

$L_{B}$

$h$

$M$

$m$

$\dot{m}$

$M w$

$N$

$n$

$P$

PR
Area

Speed of sound

Flow nozzle diameter ratio

Impeller blade height

Absolute velocity

Flow nozzle discharge coefficient (Eqn. 4.2)

Static pressure coefficient $=\frac{P_{s}-P_{s 1}}{P_{01}-P_{s 1}}$

Specific heat at constant pressure

Specific heat at constant volume

Diameter

Hydraulic diameter

Surface roughness

Impeller axial length

Vane length along mean camber line

Specific enthalpy

Mach number

Mass fraction

Mass flow rate

Molecular weight

Rotational speed

Reynolds number correction exponent

Pressure

Total pressure ratio $\mathrm{m}^{2}$

$\mathrm{m} / \mathrm{s}$

(none)

m

$\mathrm{m} / \mathrm{s}$

(none)

(none)

$\mathrm{kJ} / \mathrm{kgK}$

$\mathrm{kJ} / \mathrm{kgK}$

m

m

$\mathrm{m}$

m

m

$\mathrm{kJ} / \mathrm{kg}$

(none)

(none)

$\mathrm{kg} / \mathrm{s}$

$\mathrm{kg} / \mathrm{kmol}$

RPM

(none)

$\mathrm{Pa}$, psi

(none) 


$\begin{array}{lll}\Delta P & \text { Flow nozzle differential pressure } & \mathrm{Pa}, \mathrm{psi} \\ Q & \text { Volume flow rate } & \mathrm{m} 3 / \mathrm{s} \\ R & \text { Gas constant } & \mathrm{kJ} / \mathrm{kgK} \\ R F & \text { CFX-BladeGen+ refinement factor } & \text { (none) } \\ r & \text { Radius dimension } & \mathrm{m} \\ R e_{b 2} & \text { Reynolds number }=\frac{\rho_{01} U_{2} b_{2}}{\mu} & \text { (none) } \\ R_{U} & \text { Universal gas constant }(8.314 \mathrm{~kJ} / \mathrm{kgK}) & \mathrm{kJ} / \mathrm{kgK} \\ R H & \text { Relative humidity } & \% \\ T & \text { Temperature } & \mathrm{K},{ }^{\circ} \mathrm{C} \\ T & \text { Power input } & \mathrm{W} \\ t & \text { Impeller blade thickness } & \mathrm{m} \\ U & \text { Impeller blade speed } & \mathrm{m} / \mathrm{s} \\ V & \text { Velocity } & \mathrm{m} / \mathrm{s} \\ W & \text { Relative velocity } & \mathrm{m} / \mathrm{s} \\ \Delta W & \text { Maximum relative velocity difference across the vane } & \mathrm{m} / \mathrm{s} \\ y+ & \text { y-plus; non-dimensional measure of the boundary layer } & \\ & \text { resolution } & (\mathrm{none}) \\ \Delta y & \text { Distance of the first node to the wall } & \mathrm{m} \\ Z & \text { Number of impeller blades } & (\mathrm{none})\end{array}$

\section{Greek Symbols:}

$\begin{array}{lll}\alpha & \text { Absolute flow angle } & \operatorname{deg} \\ \beta & \text { Relative flow angle } & \operatorname{deg} \\ \gamma & \text { Isentropic exponent } & \text { (none) } \\ \eta & \text { Isentropic efficiency } & \% \\ \theta & \text { Circumferential dimension } & \operatorname{deg} \\ \lambda & \text { Colebrook friction factor } & \text { (none) }\end{array}$


$\Pi_{m} \quad$ Non-dimensional mass flow parameter

$$
\Pi_{m}=\frac{\dot{m} \sqrt{T_{01}}}{P_{01}}\left(\frac{1}{D^{2}} \sqrt{\frac{R}{\gamma}}\right)
$$

$\Pi_{N} \quad$ Non-dimensional speed parameter

$$
\Pi_{N}=\frac{N}{\sqrt{T_{01}}}\left(\frac{D}{\sqrt{\gamma R}}\right)
$$

$\rho$

$\sigma$

$\tau$

\section{Subscripts:}

Density

Slip factor $=\frac{C_{\theta 2}}{C_{\theta 2}^{\prime}}$

Tip clearance height

Wall shear stress

Work coefficient $=\Delta h_{0} / U_{2}^{2}$

Specific humidity $\mathrm{kg} / \mathrm{m}^{3}$

(none)

m

$\mathrm{N} / \mathrm{m}^{2}$

(none)

(none)

Stagnation condition

Inlet condition for compressor, impeller or volute

Outlet condition for compressor, impeller or volute

Ambient condition

Critical value

Condition at impeller hub

Radial component (of velocity)

Relative flow condition

Condition at impeller shroud, static conditions

Saturation value 
$\mathrm{t} \quad$ Test condition

TE Condition at impeller trailing edge

X Axial component (of velocity)

$\theta \quad$ Tangential or circumferential component (of velocity)

\section{Superscripts:}

Ideal condition or blade metal angle
$* \quad$ Condition at choking




\subsection{INTRODUCTION}

The technology advance of gas turbine design has led to the use of gas turbine engines in many sectors. Typically gas turbine engines used for power generation are large, simple cycle engines that are relatively clean burning. Within the power generating sector their has been a recent initiative to reduce carbon dioxide $\left(\mathrm{CO}_{2}\right)$ emissions from all fossil fuel burning power plants including those powered by gas turbine engines. Due to this initiative gas turbine designers have begun to develop technologies to reduce $\mathrm{CO}_{2}$ emissions from fossil fuel fired gas turbine engines.

The recent desire to reduce $\mathrm{CO}_{2}$ emissions from fossil fuel burning power plants has led to several projects at Carleton University focused on developing the technology for gas turbines using alternative working fluids. One such project is the Raven ZeroEmissions Gas Turbine (RZEGT) project. The aim of the RZEGT project is to design a closed-cycle gas turbine engine for power generation using $\mathrm{CO}_{2}$ as the working fluid. By using $\mathrm{CO}_{2}$ as the working fluid the excess $\mathrm{CO}_{2}$ produced during combustion can easily be bled from the system without the need for additional high energy devices. However, the closed-cycle gas turbine using $\mathrm{CO}_{2}$ as the working fluid requires additional hardware which increases the cost and complexity of the engine. The use of an alternative working fluid also affects the aerodynamic performance of the turbomachinery. To date not all effects are fully understood and it was selected to further investigate the effect of fluid properties on the compressor. The investigation was carried out both experimentally and computationally using two centrifugal compressors.

The experimental investigations of this study utilized an existing rotating test rig located in the High Speed Wind Tunnel facility at Carleton University. The test rig is capable of providing overall stage performance data for a centrifugal compressor and has the ability to run in closed-cycle configuration such that the compressor can be tested in alternative working fluids. It was originally desired to obtain experimental data and 
detailed aerodynamic performance predictions for the RZEGT compressor. To meet specific manufacturing constraints and to be able to be installed in the existing rotating test rig the compressor of the RZEGT project was re-designed as part of the present study. Throughout this work the re-designed compressor is referred to as the test rig compressor in reference to its intended use and to differentiate it from the original RZEGT compressor. During the design stage the test rig impeller was also used to investigate the influence of working fluid on the impeller design geometry. With manufacturing complete, the test rig impeller was ready for experimental testing and the results could be used to validate corresponding CFD predictions for the same geometry.

Detailed aerodynamic predictions of the test rig compressor were performed and are presented herein. However, due to time constraints the experimental testing of the test rig compressor could not be performed. Instead experimental data were collected and are presented for an existing Turbonetics T04E-50 compressor. The T04E-50 compressor was selected because it had previously been installed in the rotating test rig. In addition, a fully three-dimensional scan of the co-ordinates of the T04E-50 impeller was conducted and an accurate solids model created. The T04E-50 impeller could therefore be modelled within commercially available CFD software and the results could be compared to the experimental data. The comparison of experimental data to CFD predictions for the same geometry could therefore be used to validate the computational methods used. In preparation for future experimental testing of the test rig compressor modifications were made to the rotating test rig. These modifications included the addition of rotatable total pressure probes near the impeller outlet. The new probes made it possible to obtain estimates of the total pressure and flow angle near the impeller exit and thus the impeller alone performance could be estimated.

The chapters of this thesis are arranged as follows. Chapter 2 presents an overview of previous literature which is pertinent to the present work. This is followed by Chapters 3 and 4 which describe the experimental apparatus and the methods of 
measurement and data reduction respectively. Chapter 5 describes the computational methods used. This includes an overview of the CFD software and how each computational case was defined. The design process of the test rig compressor and the investigation of the effects of fluid properties on the impeller design geometry are both discussed in Chapter 6. Chapter 7 discusses the experimental and computational performance estimates of the Turbonetics T04E-50. The results obtained from the new instrumentation added during the test rig modifications are also presented here. The detailed aerodynamic predictions of the test rig compressor are discussed in Chapter 8 . The performance of the test rig impeller was predicted using commercially available CFD software for the complete stage and the impeller alone. Lastly, Chapter 9 presents the conclusions and recommendations for future work. 


\subsection{LITERATURE REVIEW}

\subsection{INTRODUCTION}

As mentioned in Chapter 1 , the present study involved the partial aerodynamic redesign of a centrifugal compressor impeller that had previously been designed for use in a small zero-emission gas turbine engine. In addition, in Chapter 6 the influence of varying some of the key geometric parameters on the rotor aerodynamics, including the effect of changing the working fluid, is investigated computationally. Therefore, as background for both the re-design and parametric studies, Section 2.2 summarizes the theoretical and empirical basis for the preliminary aerodynamic design of centrifugal compressors. The focus is primarily on the impeller design.

The compressor for the zero-emission gas turbine engine mentioned previously is designed using $\mathrm{CO}_{2}$ as the working fluid. Additionally, the influence of changing the working fluid on the impeller design geometry is investigated in Chapter 6. Therefore, Section 2.3 presents an overview of previous studies which investigated zero-emission gas turbine cycles. Although a brief description of other methods is given, the focus is on closed-cycle gas turbines which use alternative working fluids. Because of their design the turbomachinery of these cycles will be operating in an alternative working fluid. Therefore, Section 2.3 also reviews previous studies which investigated the influence of working fluid on the aerodynamic performance of the turbomachinery.

\subsection{Centrifugal Compressor Design}

The design of a centrifugal compressor begins from the selection of achievable design goals. These would include performance targets such as total pressure rise, efficiency, flow rate and operating range. Depending on the intended application the design goals may also include the preliminary cost, manufacturability and wear resistance 
to name a few. The design goals are potentially numerous and trade-offs must be made between desired goals early in the design stage. Once the design goals have been selected the preliminary sizing can commence. Although the focus of this review is on impeller design, it is noted that adequate compressor design systems should produce preliminary designs for the complete stage by optimizing all components and matching them to one another (Aungier, 2000). Thus the impeller design is not independent and should progress incrementally with all other compressor components.

Traditionally the design of a centrifugal compressor impeller is divided into three separate segments. The three segments are representative of three zones within the impeller: the inlet, the vane passage and the outlet. Figure 2.1 shows these regions and several important geometric properties on a generic impeller schematic.

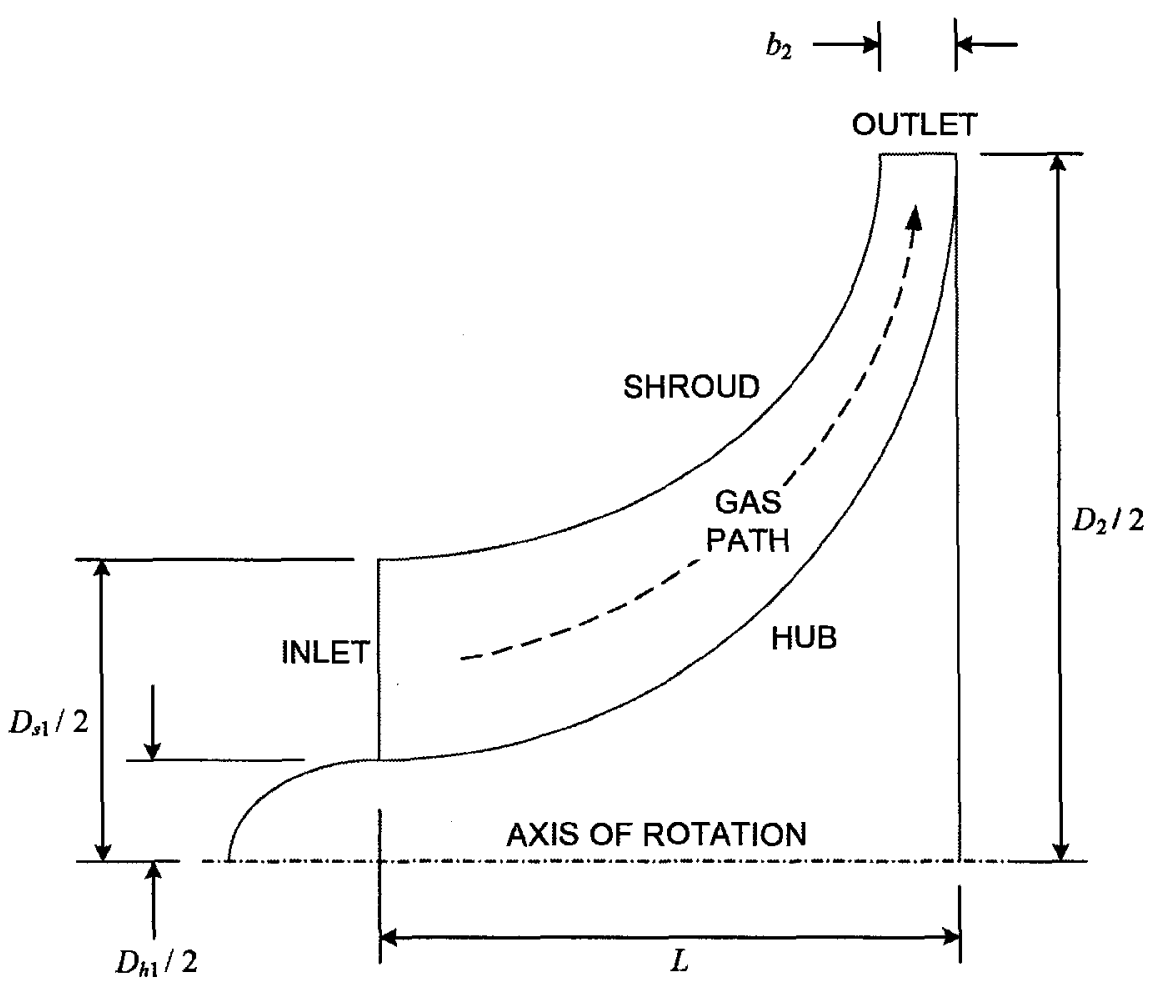

Figure 2.1: Impeller schematic 
The impeller inlet is generally designed to minimize the relative velocities at the leading edge. Authors such as Shepherd (1956), Japikse and Baines (1997), Dixon (1998), Wilson and Korakianitis (1998) and Saravanamuttoo et al. (2001) explain why better efficiency will be achieved if the inlet relative Mach number is minimized. The inlet velocity triangle of an impeller is shown in Figure 2.2. The inlet flow is assumed to have no swirl to simplify the analysis. Although an inlet swirl is sometimes used in compressors it requires the use of pre-swirl vanes (Japikse and Baines, 1997). The preswirl vanes increase the complexity of the machine and are implemented when Mach number limits cannot be met with axial inflow (Saravanamuttoo et al., 2001).

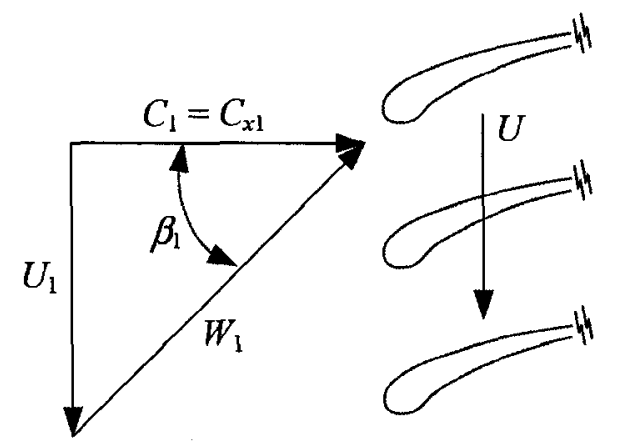

Figure 2.2: Impeller inlet velocity triangle

From Figure 2.2 the magnitude of the relative velocity $W=\sqrt{C^{2}+U^{2}}$, where $C$ is the absolute velocity and the blade speed $U=\pi D N / 60$ with $N$ in RPM. It is clear that the minimum relative velocity is therefore dependent on both the blade speed and axial velocity $\left(C_{x I}\right)$. If a compressor is operating with well designed upstream ducting the absolute flow velocity at the inlet should be constant. Since highest blade speeds occur at the largest diameter the maximum Mach numbers will be located at the shroud. The relative velocity at the shroud is:

$$
W_{s 1}=\sqrt{C_{x 1}^{2}+\left(\frac{\pi D N}{60}\right)^{2}}
$$


From continuity the axial velocity can be expressed as a function of the volume flow rate and the inlet diameters as follows:

$$
\begin{gathered}
\qquad=A_{1} C_{x 1} \\
\text { where } \quad A_{1}=\pi\left(\frac{D_{s 1}^{2}-D_{h 1}^{2}}{4}\right) \\
\text { therefore } \quad C_{x 1}=\frac{4 Q}{\pi\left(D_{s 1}^{2}-D_{h 1}^{2}\right)}
\end{gathered}
$$

Substituting Eqn. 2.2 into 2.1 gives the relative velocity expressed as a function of the flow rate, rotational speed and the inlet diameters.

$$
W_{s 1}=\left[\left(\frac{4 Q}{\pi\left(D_{s 1}^{2}-D_{h 1}^{2}\right)}\right)^{2}+\left(\frac{\pi D_{s 1} N}{60}\right)^{2}\right]^{\frac{1}{2}}
$$

Generally the hub diameter $\left(D_{h l}\right)$ is as small as possible with the limiting factor being the shaft size. In cases where the shaft size is not a factor, the hub radius should be large enough to prevent the leading edge of the vane from being highly twisted. For open-cycle gas turbine compressor the inlet density will be a function of the atmospheric conditions and will depend on the desired application. For closed-cycle gas turbine compressors the inlet pressure and therefore inlet density can be a wide variety of values. However, the inlet conditions would typically be stated in the design goals and depend on the application. Additionally, the flow rate $(Q)$ and rotational speed $(N)$ are usually specified in the design goals. A minimum inlet relative velocity is therefore achieved by the appropriate selection of the shroud diameter $\left(D_{s 1}\right)$. A large shroud diameter implies a large inlet area and thus a small axial component of velocity but a large blade speed. A small shroud diameter or small inlet area results in a large axial component of velocity 
and small blade speed. The selection of a shroud diameter at either extreme will result in larger values of relative velocity. Thus to obtain the minimum relative velocity the appropriate shroud size lies somewhere in the middle.

In nearly all compressor designs, except for those with the lowest pressure ratios, compressibility effects will be present. A similar procedure can be performed for compressible flow and is shown in Dixon (1998). The resulting expression relates the inlet relative Mach number at the shroud $\left(M_{r e l, s 1}\right)$ to the inlet gas properties, mass flow rate $(\dot{m})$, rotational speed $(N)$ and relative flow angle at the shroud $\left(\beta_{s 1}\right)$ as follows:

$$
\begin{aligned}
& \frac{\dot{m} N^{2}}{\pi k \mathcal{P}_{01} \sqrt{\gamma R T_{01}}}=\frac{M_{r e l, s 1}^{3} \sin ^{2}\left(\beta_{s 1}\right) \cos \left(\beta_{s 1}\right)}{\left[1+\frac{1}{2}(\gamma-1) M_{r e l, s 1}^{2} \cos ^{2}\left(\beta_{s 1}\right)\right]^{\frac{1}{\gamma-1}+1.5}} \\
& \text { where } \quad k=1-\left(\frac{D_{h 1}}{D_{s 1}}\right)^{2}
\end{aligned}
$$

Again the flow rate, rotational speed and inlet properties are likely specified in the design goals. To make use of Eqn. 2.4 a design decision for a desirable Mach number limit is required. An early guideline provided by Shepherd (1956) notes that it is desirable to limit the Mach number to 0.7. In applications such as superchargers and aircraft engines size and weight are valued more than efficiency, up to a certain point. In these applications Mach numbers of 0.85 are common and adequate efficiency levels can still be achieved (Shepherd, 1956). Since the work of Shepherd (1956) inlet Mach numbers have increased. However, if one wants to prevent shock waves at the inlet the Mach number should be limited to approximately Mach 1.0. More recent work by Rodgers (2005) has shown industrial machines with high pressure ratios (approximately 8.0) have inlet Mach numbers greater than 1.0. Although the study was performed to highlight the limited flow range of such compressors it still showed that operating point 
isentropic efficiency of approximately $80 \%$ can be achieved with such high inlet Mach numbers. In compressors of high pressure ratio the diffuser flow is typically supersonic and the diffuser losses affect the efficiency more than the inlet losses. Since shock losses are typically small at Mach numbers less than 1.15 it may be desirable to increase inlet Mach numbers slightly above Mach 1.0 to reduce more significant losses in the diffuser. However, shock losses increase significantly at Mach numbers greater than 1.15 and more detailed design tools would be required to investigate the trade-off between inlet and diffuser losses for these high Mach number compressors.

The outlet geometry is a function of the required work input or pressure rise of the impeller. The Euler turbomachinery equation states

$$
\Delta h_{o}=U_{2} C_{\theta 2}-U_{1} C_{\theta 1}
$$

where $\Delta h_{o}$ is the specific work input, which is related to the pressure rise, and $C_{\theta 2}$ is the outlet tangential velocity. Again the assumption of axial flow at the inlet gives $C_{\theta I}=0$ and thus $\Delta h_{o}=U_{2} C_{\theta 2}$. The selection of the blade speed and the outlet tangential velocity is therefore based on the specified pressure rise. Figure 2.3 shows the ideal outlet velocity triangle of an impeller. In ideal conditions the relative flow angle $\beta_{2}$ is assumed equal to the outlet blade angle $\beta_{2}^{\prime}$. It is clear from the outlet velocity triangle that the ideal tangential velocity is a function of the blade angle and the radial component of velocity $C_{r 2}$. From continuity, assuming uniform outlet flow from the rotor

$$
\dot{m}=\rho A V=\rho_{2} C_{r 2} A_{2}=\rho_{2} C_{r 2}\left(2 \pi r_{2} b_{2}\right)
$$

and thus for a fixed mass flow rate the outlet radial velocity is determined from the selection of the exit vane height $b_{2}$. 


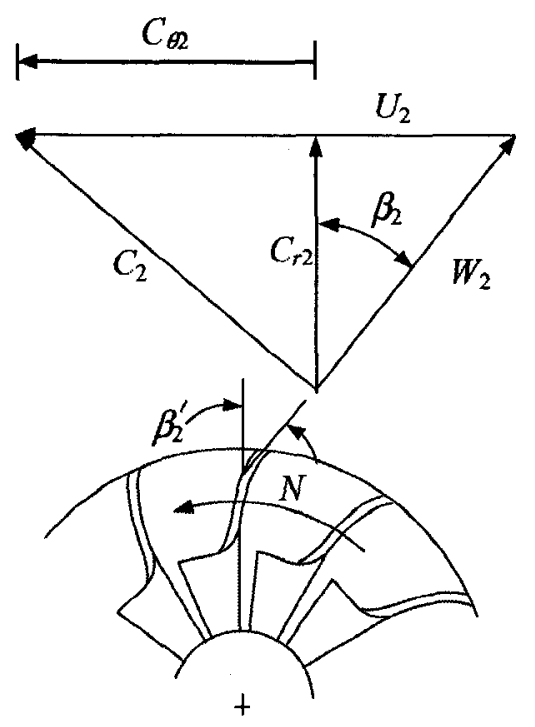

Figure 2.3: Impeller outlet velocity triangle

Figure 2.4 shows the ideal outlet velocity triangle for various exit vane heights at a constant blade speed. The outlet metal angle is adjusted to maintain a constant $C_{\theta 2}$ and thus constant pressure rise.

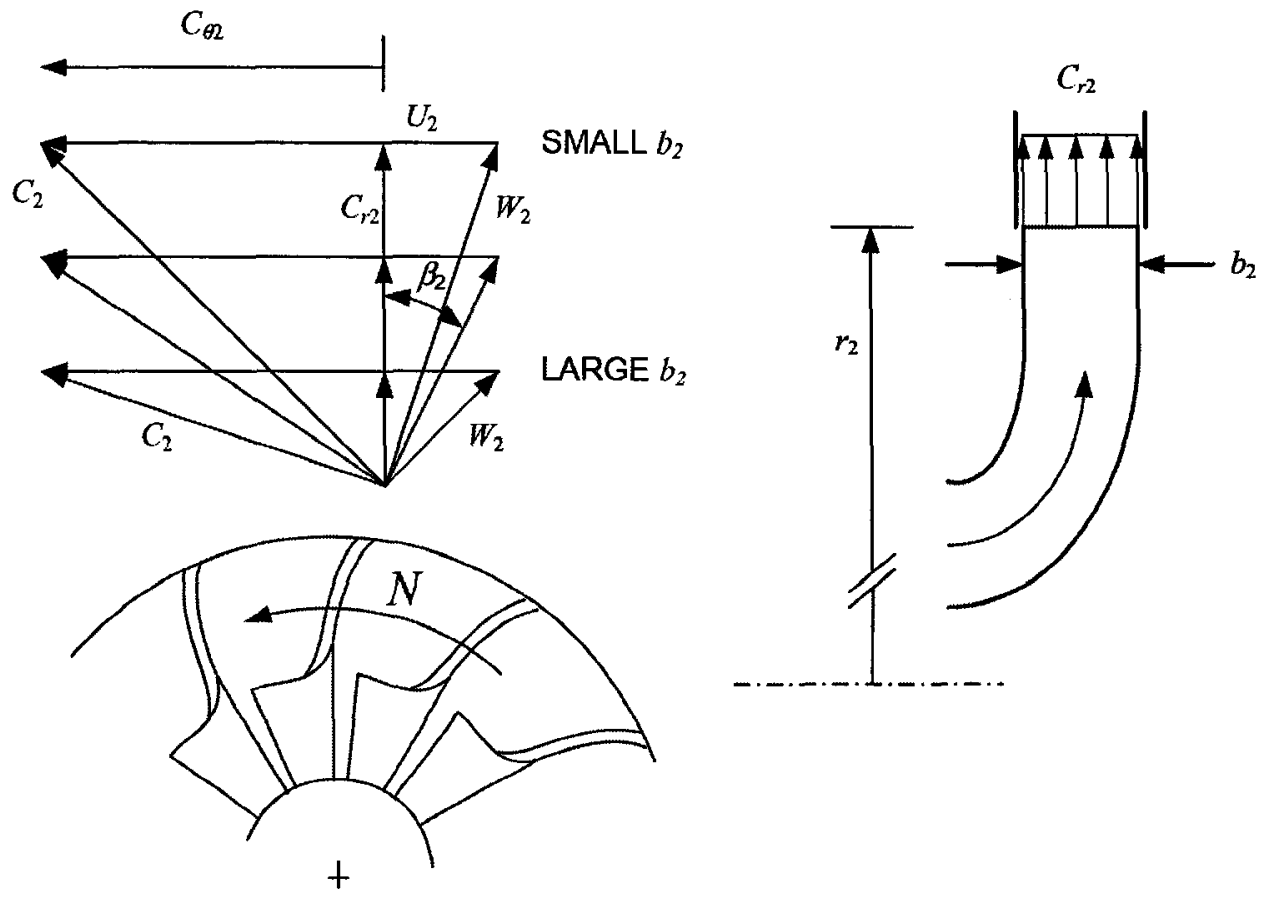

Figure 2.4: Effect of exit vane height on outlet velocity triangle 
As seen in Figure 2.4 a reduced exit vane height produces a larger absolute and relative outlet velocity. A large relative outlet velocity implies lower diffusion within the rotor passage but a large absolute outlet velocity requires more diffusion in the downstream components. The selection of the vane height would thus be chosen as a trade-off between diffusion in the rotor passage and diffusion in the downstream diffuser. Various authors provided guidelines for selecting the diffusion level in the rotor passage by quoting a minimum de Haller number. The de Haller number is defined as $W_{2} / W_{1}$ where $W_{1}$ is the relative velocity at the inlet mean radius. Table 2.1 summarizes the recommendations from several authors.

\begin{tabular}{|l|l|l|}
\hline Aungier (2000) & $\begin{array}{l}\text { Recommended } \\
\text { Lower limit }\end{array}$ & $\begin{array}{l}W_{2} / W_{1}>0.75 \\
W_{2} / W_{1}<0.65\end{array}$ \\
\hline Wilson \& Korakianitis (1998) & Recommended & $W_{2} / W_{1}>0.80$ \\
\hline Rodgers (1978) & Recommended & $W_{2} / W_{1}>0.71$ \\
\hline Yoshinaga (PWC document, 1982) & $\begin{array}{l}\text { Low PR compressors and fans } \\
\text { High PR compressors (up to 8.0) }\end{array}$ & $\begin{array}{l}W_{2} / W_{1}>0.80 \\
W_{2} / W_{1}>0.60\end{array}$ \\
\hline
\end{tabular}

Table 2.1: Suggested de Haller number limits (from Sjolander, 2008)

From the ideal velocity triangles in Figure 2.4 it is clear that the required outlet flow angle $\beta_{2}$ and corresponding metal angle $\beta_{2}^{\prime}$ is related to the selection of the outlet vane height. However, in compressors the actual outlet flow angle deviates from the metal angle. In centrifugal compressors this is due to slip. Figure 2.5 shows the effect of slip on the outlet velocity triangle. Recall that in the ideal case $\beta_{2}$ equals $\beta_{2}^{\prime}$. As seen in Figure 2.5, slip prevents the rotor from imparting the ideal swirl $C_{\theta 2}^{\prime}$ to the outlet velocity. From the Euler equation (Eqn. 2.5 ) it is clear that $\Delta h_{0}$ and therefore the pressure rise is dependent on $C_{\theta 2}$. Thus to obtain the required pressure ratio an accurate estimate of the slip is needed. 


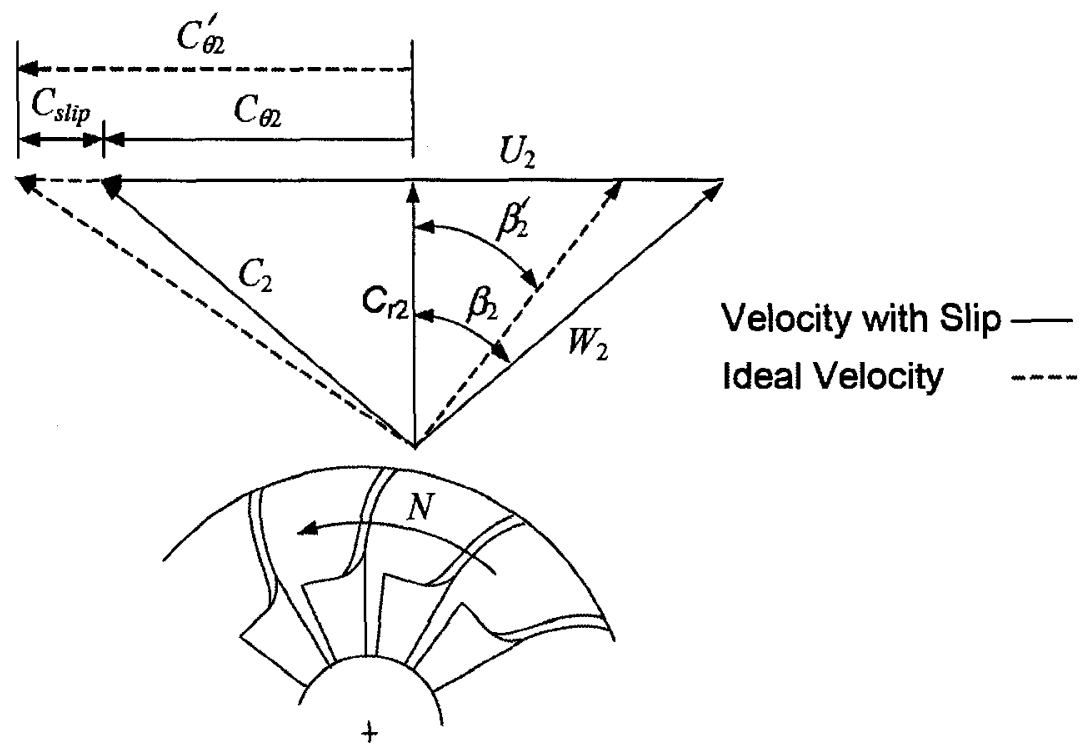

Figure 2.5: Effect of slip on outlet velocity triangle

The slip is quantified by the slip factor $(\sigma)$ defined as

$$
\sigma=1-\left(C_{s i p} / C_{\theta 2}^{\prime}\right)
$$

or equivalently

$$
\sigma=C_{\theta 2} / C_{\theta 2}^{\prime}
$$

Several authors have generated empirical formulations based on the approximate analysis of the secondary flows within the rotor passage. Some well known formulations are due to Stodola (1927), Busemann (1928) and Stanitz (1952). Each formulation has proved its capability of predicting the slip factor accurately and their definitions are still referenced in modern turbomachinery texts such as Saravanamuttoo, et al. (2001), Japikse and Baines (1997), Dixon (1998) and Wilson and Korakianitis (1998). A widely accepted correlation for predicting the slip factor of a compressor impeller with long vanes is shown in Eqn. 2.9 and is provided by Wiesner (1967). 


$$
\sigma=1-\frac{\sqrt{\cos \beta_{2}^{\prime}}}{Z^{0.7}}
$$

where $Z$ is the number of blades. Wiesner (1967) also provides a correction factor to Eqn. 2.9 for use with shorter vanes and is based on the radius ratio $\varepsilon=r_{1} / r_{2}$.

A broad guideline for selecting the number of blades as a function of the outlet metal angle is given in Wilson and Korakianitis (1998). Since the authors present only a graphical representation of their recommendation it has not been reproduced here.

Aungier (2000) provides a guideline for selecting the number of vanes based on a recommended vane loading limit, or

$$
\frac{2 \Delta W}{W_{2}+W_{1}} \leq 0.9
$$

where $\Delta W$ is the maximum relative velocity difference across the vane and can be estimated from

$$
\Delta W=\frac{2 \pi D_{2} U_{2} \psi}{2 L_{B}}
$$

where the work coefficient, $\psi=\Delta h_{0} / U_{2}^{2}$ and $L_{B}$ is the vane length along the mean camber line. An initial estimate of $L_{B}$ is found from

$$
L_{B}=\left(L-\frac{b_{2}}{2}\right)+\frac{1}{2}\left(\frac{D_{2}-D_{1}}{\cos \beta_{2}^{\prime}}\right)
$$


The application of Equations 2.1 to 2.12 provides a broad guideline for a preliminary design of the inlet and outlet geometry based on the specified design goals. The final stage in the preliminary design is defining the vane passage through the impeller. The vane passage is defined by the geometry of the surfaces that bound the passages through which the fluid flows. In an impeller the bounding surfaces are the hub, shroud and blades. For un-shrouded impellers the shroud surface is defined on the compressor casing. The vane tips will follow a similar curve with a specified clearance from the shroud to allow rotation. The passage flow of a centrifugal compressor is threedimensional and thus very complex. Wilson and Korakianitis (1998) suggest that the science of centrifugal machine design is complemented by an art that is contributed to by a team of designers with years of experience. Nevertheless, the analysis of the impeller passage flow has yielded several design methods which are suitable guides for vane passage designs. Saravanamuttoo et al. (2001) refers to an early method by Came (1978) which makes use of the Marsh 'matrix throughflow' aerodynamic analysis procedure to determine the shape of the impeller channels. Aungier (2000) provides several more recent methods for defining the surface geometries based on different curve forms. Each curve form method that is reviewed also includes a discussion of its advantages and disadvantages.

A need for high pressure ratio single-stage transonic centrifugal compressors has been created by the recent demand for increased thermal efficiency and power density in the aviation and energy industries (Rodgers, 2005). The high Mach numbers and potential for shock waves adds to the already complex flow within the impeller passages. Thus the design performance of a transonic centrifugal compressor cannot be guaranteed from the traditional design method discussed previously. Until recently the typically small vane passages of a transonic centrifugal compressor have prevented the detailed experimental analysis of the inter passage flow. With advances in computational fluid dynamics (CFD) more detail of the rotor passage flow has become available. Kang (2005), Marconcini et al. (2006) and Krain et al. (2007) all conducted numerical 
investigations of the passage flow in high speed centrifugal compressors. In all three studies validation of the computations was obtained by comparing the efficiency and pressure ratio predicted by CFD to experimental data. The work by Kang (2005) provides insight into the complex shock wave system inside the blade passage and its interaction with vortices and boundary layers. It concludes that the three-dimensional viscous flow structure, energy loss production and velocity patterns are greatly affected by the shock waves in the inlet region while the remaining regions have similar flow structures. Marconcini et al. (2006) investigated the capabilities of current computational methods. They focused primarily on how the tip gap and turbulence modeling affects the predicted inter passage flow and compressor performance. Krain et al. (2007) investigated a new design method and details of the passage flow obtained experimentally were used to validate a new high pressure ratio compressor design. For this purpose Krain et al. (2007) conducted laser Doppler velocity measurements near the impeller exit to analyze the flow pattern.

In addition to technology advances in high pressure ratio transonic machines, there is still ongoing work improving the traditional design methods and analysis of centrifugal compressors. Del Greco et al. (2007) presented a new slip factor correlation for centrifugal impellers for a wide range of flow coefficients and blade Mach numbers. The improvement over classical formulations such as Wiesner (1967) is evident. However, the correlation of Del Greco et al. (2007) is limited to the flow coefficient and blade Mach number ranges investigated. Compressor stages beyond the scope of the study, in particular at very high blade Mach numbers, still represent a challenge for the correlation. An improved slip factor model was also presented by Qiu et al. (2007). In their study Qiu et al. attempted to unify the deviation models of axial machines and slip factor correlations of centrifugal impellers into a single model. 


\subsection{Alternative WORKIng FLUids For Gas TuRbine CyCLES}

With ever increasing environmental pressures new technologies are being developed in the gas turbine power generation sector to reduce the emission of greenhouse gases, and in particular carbon dioxide. Early methods developed focused on capturing $\mathrm{CO}_{2}$ from the exhaust of traditional fossil fuel combustion processes that use air as the oxidant. However, the increased capital cost and energy usage required for the separation of $\mathrm{CO}_{2}$ from the exhaust gasses has led to the general consensus that $\mathrm{CO}_{2}$ recovery is not feasible in current power plants (Wang et al., 2004). Instead more promising methods that do not rely on post combustion clean-up have started to be investigated.

Although many new methods of $\mathrm{CO}_{2}$ recovery have been proposed, essentially all can be categorized into two types. In one type the hydrocarbon fuel, either solid or liquid, is pre-processed to remove the carbon. This process known as gasification results in a hydrogen rich fuel known as syngas and concentrated levels of $\mathrm{CO}_{2}$ which can be relatively easily removed. The syngas can then be used in the combustion chamber of a gas turbine resulting in very little $\mathrm{CO}_{2}$ in the exhaust. This process is regarded as being the most promising way to generate power from coal when integrated into a combined cycle (Corradetti and Desideri, 2004). In the other $\mathrm{CO}_{2}$ recovery method combustion occurs at near stoichiometric conditions with pure oxygen $\left(\mathrm{O}_{2}\right)$ as the oxidizer such that the products of combustion are only $\mathrm{CO}_{2}$ and water. These are often known as oxy-fuel cycles. If the cycle working fluid is $\mathrm{CO}_{2}$, as proposed by De Ruyck (1992) and Mathieu et al. (2004), the excess $\mathrm{CO}_{2}$ produced from combustion can be captured and sequestered. The use of $\mathrm{CO}_{2}$ as the working fluid would require closed-cycle operation of the gas turbine. The water also produced by combustion is condensed and removed and the $\mathrm{CO}_{2}$ working fluid is cooled and recycled through the engine. The use of a closed-cycle gas turbine with $\mathrm{CO}_{2}$ as the working fluid raises the question of the effect of the working 
fluid on the aerodynamic design and performance of the turbomachinery. This question is the focus of the present research.

The cycle schematic of the closed-cycle gas turbine for power generation using $\mathrm{CO}_{2}$ as the working fluid with $\mathrm{CO}_{2}$ recovery, as proposed by De Ruyck (1992) and Mathieu et al. (2004), is shown in Figure 2.6. As mentioned, oxygen and a clean burning fuel such as methane $\left(\mathrm{CH}_{4}\right)$ enter the combustor at near stoichiometric conditions such that the products of combustion are essentially water and $\mathrm{CO}_{2}$. The excess $\mathrm{CO}_{2}$ can then be bled from the system requiring little or no additional energy depending on whether additional compression is required for storage of the $\mathrm{CO}_{2}$. However, additional energy is needed to operate the air separation unit (ASU) to produce the oxygen required for combustion. Losses generated within the additional components (condenser and heat exchanger) also lower the net thermal efficiency of the closed-cycle compared with the traditional open cycle using air. Regardless, it is believed that the overall efficiency of a closed-cycle can be at best as good as that of air cycle plants with $\mathrm{CO}_{2}$ recovery and the concept may be economically viable (Ulizar and Pilidis, 1999). In addition, the closedcycle plant has the advantage of allowing variation of the circuit pressure. By reducing the circuit pressure there is a corresponding decrease in density. This results in a reduction in mass flow rate which does not alter the velocity triangles of the turbomachinery. Since the power output of an engine is a function of the mass flow rate, it is therefore possible to reduce the power output during off-peak times without altering the non-dimensional operating point of the turbomachinery. Thus, the closed-cycle plant may be able to operate at peak efficiency at all times and this contributes to its economic attractiveness. However, at reduced power output the ASU would have to operate at offdesign conditions which would likely reduce the overall thermal efficiency of the engine. 


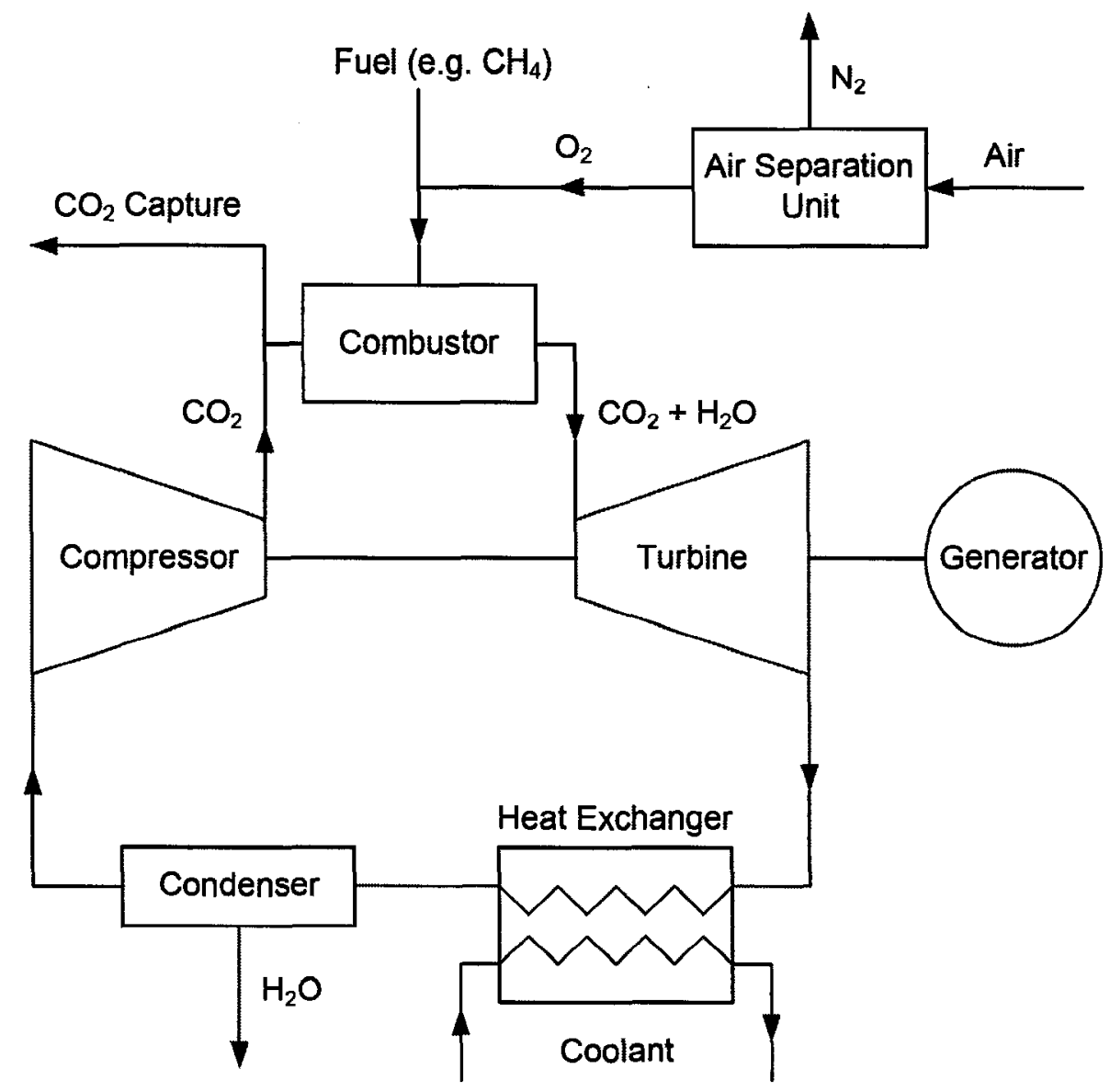

Figure 2.6: Schematic representation of a closed-cycle gas turbine for power generation with $\mathrm{CO}_{2}$ recovery

Like open cycle machines, the closed-cycle configuration also lends itself to a variety of gas turbine power plant configurations. Mathieu and De Ruyck (1993) and Jackson et al. (2000) discuss the advantages of a bottoming steam cycle integrated with the heat exchanger. The resulting combined cycle plant will have an increased thermal efficiency over the simple closed-cycle. Mathieu and De Ruyck (1993) also propose a combined $\mathrm{CO}_{2} /$ steam gas turbine cycle and steam injected gas turbine cycle (STIG) as potential solutions to counteract the power requirements of the ASU. A promising configuration based on a combined $\mathrm{CO}_{2}$ /steam cycle is being developed at the Graz University of Technology. The Graz cycle originally proposed in Jericha et al. (2002) has been continuously investigated and improved. The most current performance and 
economic analysis of the Graz cycle are discussed in Jericha et al. (2006). The authors believe that the $\mathrm{CO}_{2}$ recovery costs of the current cycle configuration are economically viable based on future $\mathrm{CO}_{2}$ emissions trading.

The use of an alternative working fluid to air is not an idea unique to reducing $\mathrm{CO}_{2}$ emissions. Harman (1981) and Saravanamuttoo et al. (2001) discuss the advantage of alternative working fluids in closed-cycle gas turbines. The authors note that there are potential benefits to using gasses with lower molecular weights. The high specific heat of such gasses would yield a gas turbine cycle with a high work output per unit mass flow. Regardless of the potential advantages of alternative working fluids there is a change in the performance of a gas turbine expected when changing working fluids. Before further discussion of the influence of alternative working fluids on gas turbine performance, the non-dimensional parameters used to express turbomachinery performance will be reviewed.

Buckingham's $\mathrm{Pi}$ theorem allows engineers and researchers to express dimensional variables of a problem in a more compact non-dimensional form. Thus independent variables are reduced to a set of independent non-dimensional parameters, or similarity criteria. All dependent non-dimensional parameters of the problem are then functions of the independent non-dimensional parameters. If all similarity criteria are matched, the dependent parameters are also equal. Buckinghams's Pi theorem gives the same number of non-dimensional parameters for a given problem as a function of the number of independent dimensional variables. However, there can be more than one possible set of the independent non-dimensional parameters depending on the variables that are chosen from the list of independent variables. Introductory fluid mechanics textbooks, such as White (1994), provide a more detailed discussion of Buckingham's Pi theorem and dimensional analysis. 
The common independent non-dimensional parameters used in turbomachinery when the working fluid is a perfect gas and compressible are the mass flow parameter $\left(\Pi_{m}\right)$, the speed parameter $\left(\Pi_{N}\right)$, the Reynolds number $\left(\operatorname{Re}_{b 2}\right)$ and the specific heat ratio $(\gamma)$ all defined here:

\section{Mass Flow Parameter:}

$$
\Pi_{m}=\frac{\dot{m} \sqrt{T_{01}}}{P_{01}}\left(\frac{1}{D^{2}} \sqrt{\frac{R}{\gamma}}\right)
$$

where $P_{01}$ and $T_{01}$ are the total pressure and total temperature at the compressor inlet. The mass flow parameter can be interpreted as the Mach number based on the axial velocity at the inlet for a compressor.

\section{Speed Parameter:}

$$
\Pi_{N}=\frac{N}{\sqrt{T_{01}}}\left(\frac{D}{\sqrt{भ R}}\right)
$$

The speed parameter can be interpreted as the Mach number based on the blade speed at the rotor tip.

Reynolds Number:

$$
R e_{b 2}=\frac{\rho_{01} U_{2} b_{2}}{\mu}
$$

As indicated, for centrifugal compressors the blade speed $\left(U_{2}\right)$ and vane height $\left(b_{2}\right)$ at the rotor tip are often used as the velocity and length scales respectively. Alternative forms of the Reynolds number are also possible. 
Specific Heat Ratio (Isentropic Exponent):

$$
\gamma=\frac{c_{p}}{c_{v}}
$$

The specific heat ratio relates changes in pressure to changes in temperature for an isentropic process of a perfect gas, or:

$$
\frac{T_{2}}{T_{1}}=\left(\frac{P_{2}}{P_{1}}\right)^{\frac{\gamma-1}{\gamma}}
$$

Since Eqn. 2.17 is for an isentropic process, $\gamma$ is often referred to as the isentropic exponent.

The dependent non-dimensional parameters are then functions of the above nondimensional independent parameters. For turbomachinery performance the dependent performance parameters most widely used are the total pressure ratio and isentropic efficiency.

\section{Total Pressure Ratio:}

The total pressure ratio is used to non-dimensionally express the change in total pressure. For a compressor it is defined as the ratio of the total or stagnation pressure at the compressor discharge to the total pressure at the inlet.

$$
P R=\frac{P_{02}}{P_{01}}
$$




\section{Isentropic Efficiency:}

The isentropic efficiency of a compressor is defined as the ratio of the ideal work input to the actual work input for the same pressure rise. The ideal work input is the amount of work required to perform an isentropic compression. For a perfect gas, with no heat transfer and constant fluid properties the efficiency can be expressed as:

$$
\eta=\frac{\left(\frac{P_{02}}{P_{01}}\right)^{\frac{\gamma-1}{\gamma}}-1}{\frac{T_{02}}{T_{01}}-1}
$$

Compressor performance is often presented in the form of compressor maps or characteristics. A generic compressor characteristic is shown in Figure 2.7. As seen a typical characteristic would plot the total pressure ratio against the mass flow rate for constant values of the rotational speed. As is the case in Figure 2.7, lines of constant efficiency are often shown; otherwise, a separate characteristic which plots efficiency against the mass flow rate for constant speeds can be used. The surge line indicates the mass flow rate at which the compressor will surge for a given speed. Surge is a system instability which is typically avoided during compressor operation and will be discussed more thoroughly in Chapter 6. If the mass flow is increased such that the velocity at the minimum throat area reaches Mach 1.0 across the entire flow passage the compressor is said to be choked and no further increase in mass flow rate is possible. This is indicated on the compressor characteristic by vertical lines of constant speed as is shown with $\mathrm{N}_{3}$ and $\mathrm{N}_{4}$. Typically the actually mass flow rate and rotational speed are not quoted on a compressor map. Instead corrected or non-dimensional parameters are used. 


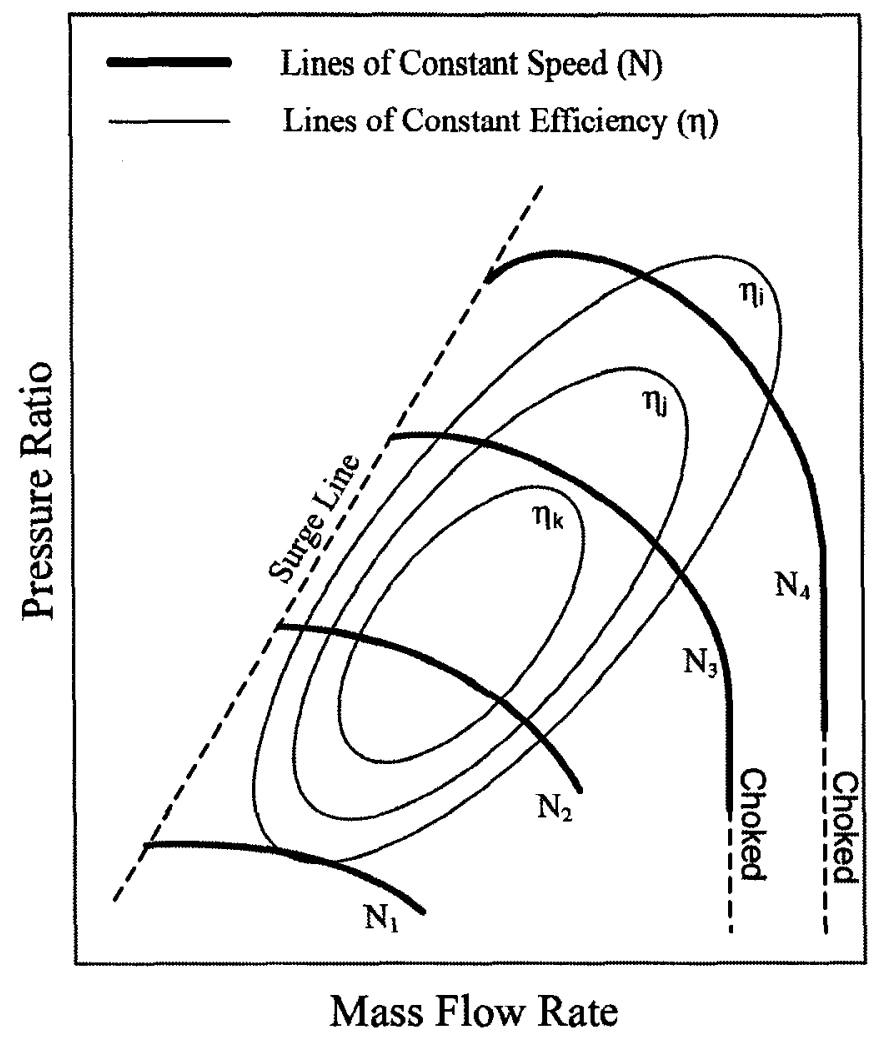

Figure 2.7: Generic compressor characteristic

The use of non-dimensional parameters on a compressor characteristic implies that the performance characteristics are only valid for matching Reynolds number and isentropic exponent. The effect of Reynolds number on the compressor performance is generally small and is quite well understood. In instances where the effect of Reynolds number needs to be accounted for there are several empirical correlations available which correct the efficiency for variations in the test and operating machine Reynolds number. One such correlation for centrifugal compressors was developed by Strub et al. (1987). The authors also provide Reynolds number corrections for the pressure ratio and flow parameter. When testing compressors with the same working fluid as used in operation the isentropic exponent is typically not included as a similarity criterion. Unlike with the Reynolds number, until recently there was little information available in the literature which discusses the influence of the isentropic exponent $\gamma$ on the compressor performance. 
When investigating the use of alternative working fluids in gas turbines there is often a change in $\gamma$ between different gasses. For example at room temperature the value of $\gamma$ for $\mathrm{CO}_{2}$ is about 1.29 compared with 1.4 for air. Since $\gamma$ is a similarity criterion there should in principle be differences in the turbomachinery performance if $\gamma$ is varied. However, many researchers assume that the isentropic exponent is not an important criterion of similarity and thus has little influence on the compressor performance. Saravanamuttoo et al. (2001) discusses the advantages of alternative working fluids by performing a theoretical analysis which compares the performance of a closed-cycle gas turbine using air and helium as working fluids. The differences in expected engine performance are noted but the change in working fluid is assumed to have no effect on the performance of the turbomachinery. Jackson et al. (2000) compare the performance of a gas turbine using air and $\mathrm{CO}_{2}$ as the working fluid in closed-cycle operation and noted the change in performance of both the engine and the turbomachinery. The authors recognized the importance of $\gamma$ in the mass flow and speed parameters and used the full non-dimensional forms shown previously. However, the authors missed the influence of $\gamma$ as a separate non-dimensional criterion.

Until the work of Roberts (2001), there appeared to be no information available in the literature which quantified the effect of the isentropic exponent on turbomachinery performance. Roberts (2001) investigated the performance of a centrifugal compressor in alternative working fluids using experimental data, computational and theoretical analysis. Roberts (2001) concluded that differences in the isentropic exponent do significantly influence the compressor performance. He also derived correlations to predict the performance of a turbomachine in an alternative working fluid based on the known performance in another fluid. The correlations developed by Roberts (2001) predict the effect of variation in the isentropic exponent on the non-dimensional mass flow parameter at choking $\left(\Pi_{m}^{*}\right)$, the total pressure ratio $\left(P_{02} / P_{01}\right)$ and isentropic 
efficiency $(\eta)$ for gas $B$ from the same performance parameters in gas $A$ for given values of the non-dimensional speed and mass flow parameter as follows:

\section{Choking Mass Flow Parameter:}

$$
\frac{\Pi_{m B}^{*}}{\Pi_{m A}^{*}}=\frac{\left(\frac{2}{\gamma_{B}+1}\right)^{\frac{1+\gamma_{B}}{2\left(\gamma_{B}-1\right)}}}{\left(\frac{2}{\gamma_{A}+1}\right)^{\frac{1+\gamma_{A}}{2\left(\gamma_{A}-1\right)}}}
$$

where $\Pi_{m}$ is the mass flow parameter and the asterisk superscript indicates the value at choking.

Isentropic Efficiency:

$$
\frac{1-\eta_{A}}{1-\eta_{B}} \cong \frac{\gamma_{A}}{\gamma_{B}}
$$

Eqn. 2.21 was derived using a number of simplifying assumptions. It was found from experimental data and computations that better results are obtained when Eqn. 2.21 takes the form:

$$
\frac{1-\eta_{A}}{1-\eta_{B}} \cong\left(\frac{\gamma_{A}}{\gamma_{B}}\right)^{n}
$$

where a value of $n$ of 0.8 appeared to work well for the limited available data (Roberts, 2001). 
Total Pressure Ratio:

$$
\frac{P_{o 2 B}}{P_{o 1 B}}=\left(1+\left(\frac{\gamma_{B}-1}{\gamma_{A}-1}\right)\left(\left(\frac{P_{o 2 A}}{P_{o 1 A}}\right)^{\frac{\gamma_{A}-1}{\gamma_{A}}}-1\right)\right)^{\frac{\gamma_{B}}{\gamma_{B}-1}}
$$

The use of Eqn. 2.23 predicts an operating point in gas $B$ with a different pressure ratio to gas $A$ but equal mass flow parameter. However, Eqn. 2.23 does not account for changes in the choking flow parameter between different gases. It was found that better results were obtained if the mass flow parameter $\Pi_{m}$ is normalized by the value of the flow parameter at choking, $\Pi_{m}^{*}$. Thus Eqn. 2.23 should predict the pressure ratio of gas $B$ from the pressure ratio of gas $A$ at a different value of $\Pi_{m}$. The value of the mass flow parameter for gas $B\left(\Pi_{m B}\right)$ is a function of the ratio of the choking mass flow parameters of the two gases, or:

$$
\frac{\Pi_{m B}}{\Pi_{m B}^{*}}=\frac{\Pi_{m A}}{\Pi_{m A}^{*}}
$$

By using Eqn. 2.24 the predicted pressure ratio of gas $B$ is unchanged. However, the predicted pressure ratio in gas $B$ is for a different value of the mass flow parameter than gas $A$.

The use of Eqn. 2.23 also neglects the effect of efficiency on the pressure ratio due to the change in working fluid. Roberts (2001) suggested that the pressure ratio of gas $B$ predicted from the pressure ratio of gas $A$ when accounting for the change in efficiency between gas $A$ and $B$ takes the form of Eqn. 2.25. 


$$
\frac{P_{o 2 B}}{P_{o 1 B}}=\left(1+\frac{\eta_{B}}{\eta_{A}}\left(\frac{\gamma_{B}-1}{\gamma_{A}-1}\right)\left(\left(\frac{P_{o 2 A}}{P_{o 1 A}}\right)^{\frac{\gamma_{A}-1}{\gamma_{A}}}-1\right)\right)^{\frac{\gamma_{B}}{\gamma_{B}-1}}
$$

where the change in efficiency is first predicted from Eqn. 2.21 or 2.22. Again, Eqn. 2.25 should be used to predict the pressure ratio of gas $B$ at a corrected mass flow parameter using Eqn. 2.24.

Roberts (2001) originally compared his correlations to experimental data and computational predictions of a centrifugal compressor from a commercially available automotive turbocharger. Within the present work the correlations by Roberts (2001) are compared to computational predictions of a newly designed centrifugal compressor. By using a different compressor geometry than originally used by Roberts the correlations should be validated further. 


\subsection{EXPERIMENTAL APPARATUS}

\subsection{TURBONETICS T04E-50 TEST COMPRESSOR}

As mentioned in Chapter 1, there are three centrifugal compressors involved in the investigations presented in this thesis:

i. The compressor designed by Charest (2003) for the zero-emission gas turbine.

ii. A re-designed version of compressor (i), which is designated the test rig compressor in this thesis.

iii. A commercial centrifugal compressor from an automotive turbocharger, a Turbonetics Model T04E-50

The original intention was to obtain experimental data for the test rig compressor. However, for reasons outlined later, this proved unfeasible. Instead, all experimental testing was conducted for the Turbonetics T04E-50 compressor, which is described in the present section. This compressor was selected because it had previously been used in the turbocharger based test rig designed by Roberts (2001). Also manufacturer's data and the experimental data previously collected by Roberts provided benchmarks for the compressor performance and the capabilities of the test rig. The geometry of the Turbonetics compressor was not specified by the manufacturer, Garret Turbocharger Systems. However, a full three-dimensional scan was performed to measure the coordinates of the impeller. Thus it was also possible to perform detailed CFD predictions for comparison with the measurements. The T04E-50 impeller geometry is shown schematically in Figure 3.1 and summarized in Table 3.1. 


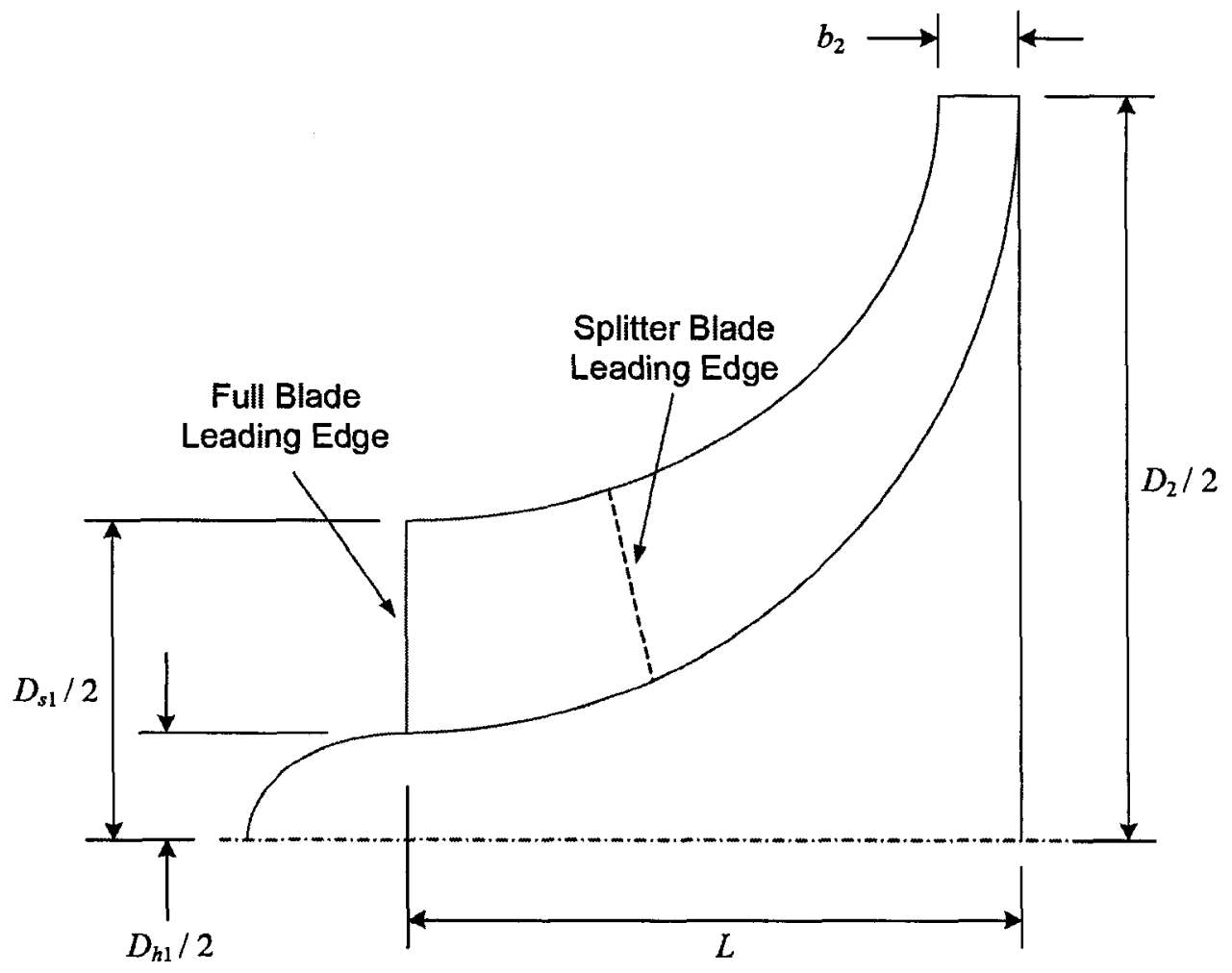

Figure 3.1: Schematic of the T04E-50 impeller (Axial cross-section)

\begin{tabular}{|c|l|c|}
\hline Symbol & Description & Dimension \\
\hline$Z$ & Number of Full Blades & 6 \\
\hline$Z_{s}$ & Number of Splitter Blades & 6 \\
\hline$D_{2}$ & Impeller Tip Diameter & $76.2 \mathrm{~mm}$ \\
\hline$b_{2}$ & Impeller Tip Vane Height & $5.1 \mathrm{~mm}$ \\
\hline$D_{s 1}$ & Inlet Shroud Diameter & $53.9 \mathrm{~mm}$ \\
\hline$D_{h 1}$ & Inlet Hub Diameter & $18.3 \mathrm{~mm}$ \\
\hline$L$ & Axial Length & $24.5 \mathrm{~mm}$ \\
\hline$\beta_{1}$ & Inlet Meanline Blade Angle & $52^{\circ}$ \\
\hline$\beta_{2}$ & Outlet Blade Angle (Backsweep) & $35^{\circ}$ \\
\hline
\end{tabular}

Table 3.1: Summary of T04E-50 impeller geometry 


\subsection{TEST APPARATUS}

\subsubsection{Test Circuit}

As mentioned, the test rig was originally designed by Roberts (2001) and a more complete description can be found there. With the exception of some modifications to the instrumentation all aspects of the original test rig have been preserved. The changes made to the instrumentation are outlined in the discussion of the measurement methods in Chapter 4.

The rotating test rig is located in the High Speed Wind Tunnel (HSWT) facility at Carleton University. The test rig is based on a turbocharger and allows overall stage performance data to be collected on the compressor of the turbocharger. The test rig utilizes high pressure air, supplied from the HSWT storage tanks, to drive the turbocharger turbine and thus the test compressor. The complete circuit of the test rig is shown schematically in Figure 3.2 for the open-circuit configuration used when the working fluid is air.

When in open-cycle configuration ambient air is collected through a bellmouth inlet and subsequently discharged to the room. All piping between instrumented stations and the compressor were insulated to minimize heat loss and the locations of the instrumentation are in accordance with the guidelines of ASME PTC 10. The compressor rotational speed is controlled by setting the blowing pressure into the turbine of the turbocharger. The flow rate and pressure ratio are controlled by one or both of the two throttle valves installed in the inlet and discharge sections of the test circuit. Throttling the inlet flow reduced the inlet density and meant less power was required for a given pressure ratio. With the duration of a run limited by the capacity of the HSWT storage tanks a reduction in power yielded an increase in run time. However, throttling the inlet also resulted in significant variations in the test Reynolds number. Although 
performance parameters were corrected for Reynolds number it was decided that the discharge valve would be used to control the pressure ratio for all test points.

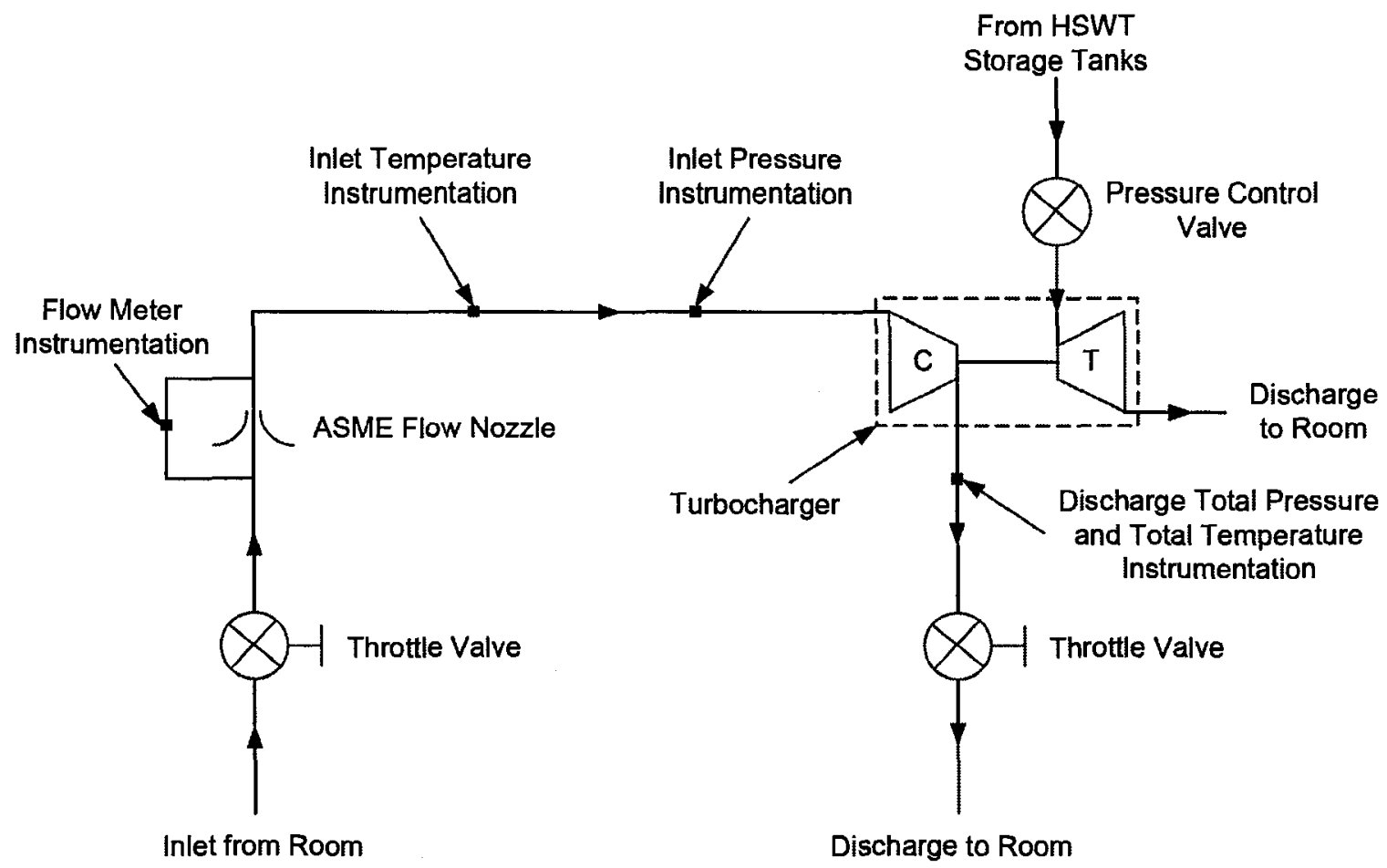

Figure 3.2: Rotating test rig circuit schematic

The test rig is also capable of running in closed-cycle configuration to measure the compressor performance with alternative working fluids. However, all the present tests were performed using air and were therefore run in open-cycle configuration. The fully assembled test rig in closed-cycle configuration is shown in Figure 3.3 (taken from Roberts, 2001). 


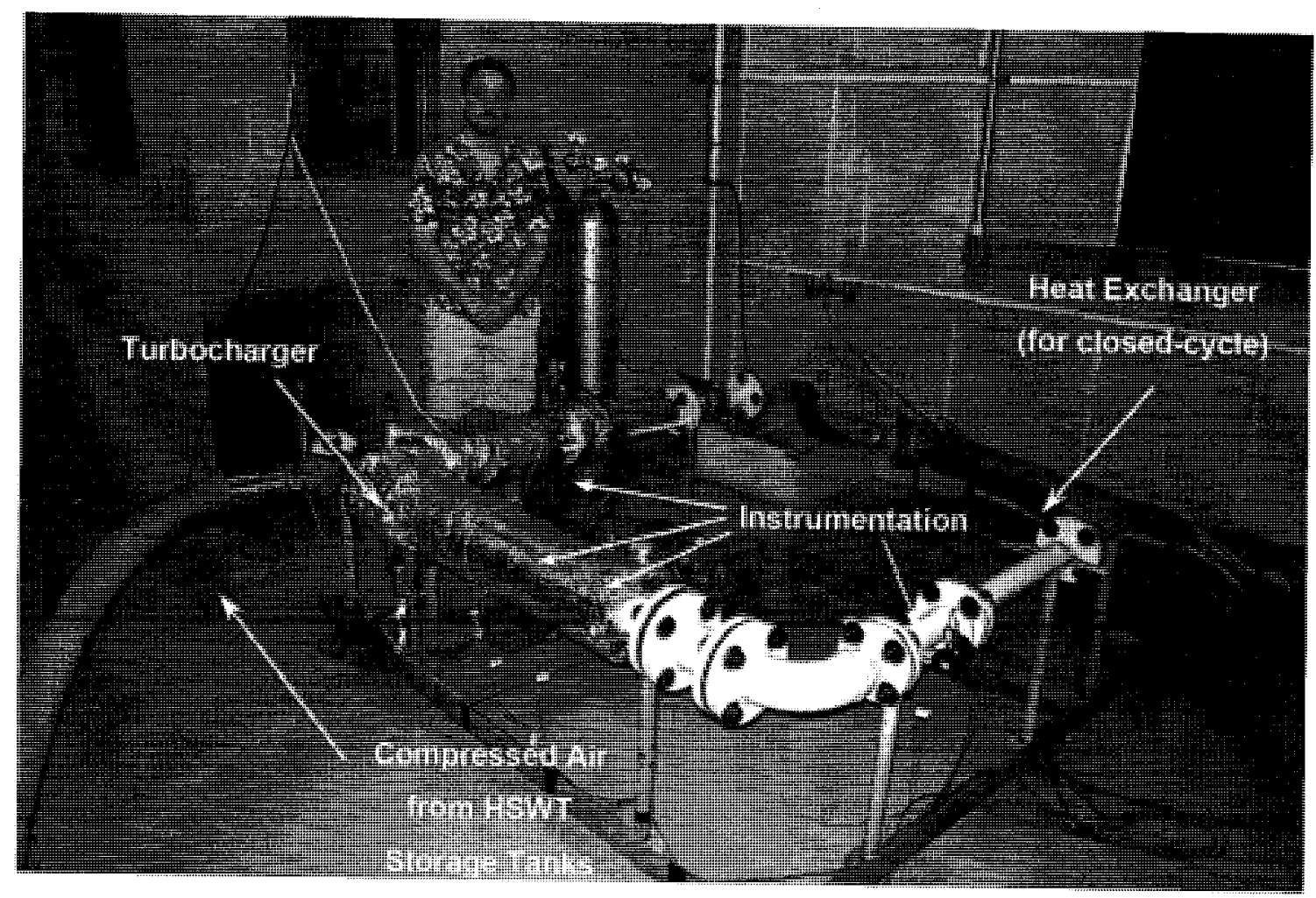

Figure 3.3: Fully assembled test rig in closed-cycle configurations (Roberts, 2001)

\subsubsection{High Speed Wind Tunnel Facility and Control System}

The HSWT facility, shown in Figure 3.4, was used to supply the high pressure air required to drive the turbocharger. Originally designed by Jeffries (1994), as a blowdown type wind tunnel for high speed testing of turbine cascades, the storage tanks are also used to supply the pressurized air for the turbocharger-based test rig.

The HSWT storage tanks are filled by a large reciprocating compressor in the basement of the building. Together, the tanks and compressor are capable of providing enough mass flow of air for continuous operation of the test compressor at low rotational speeds. At higher speeds enough pressurized air is available to maintain a constant blowing pressure on the turbocharger to provide steady-state operation of the compressor 
for the fifteen minutes or more specified in the performance test code on compressors (ASME PTC 10).

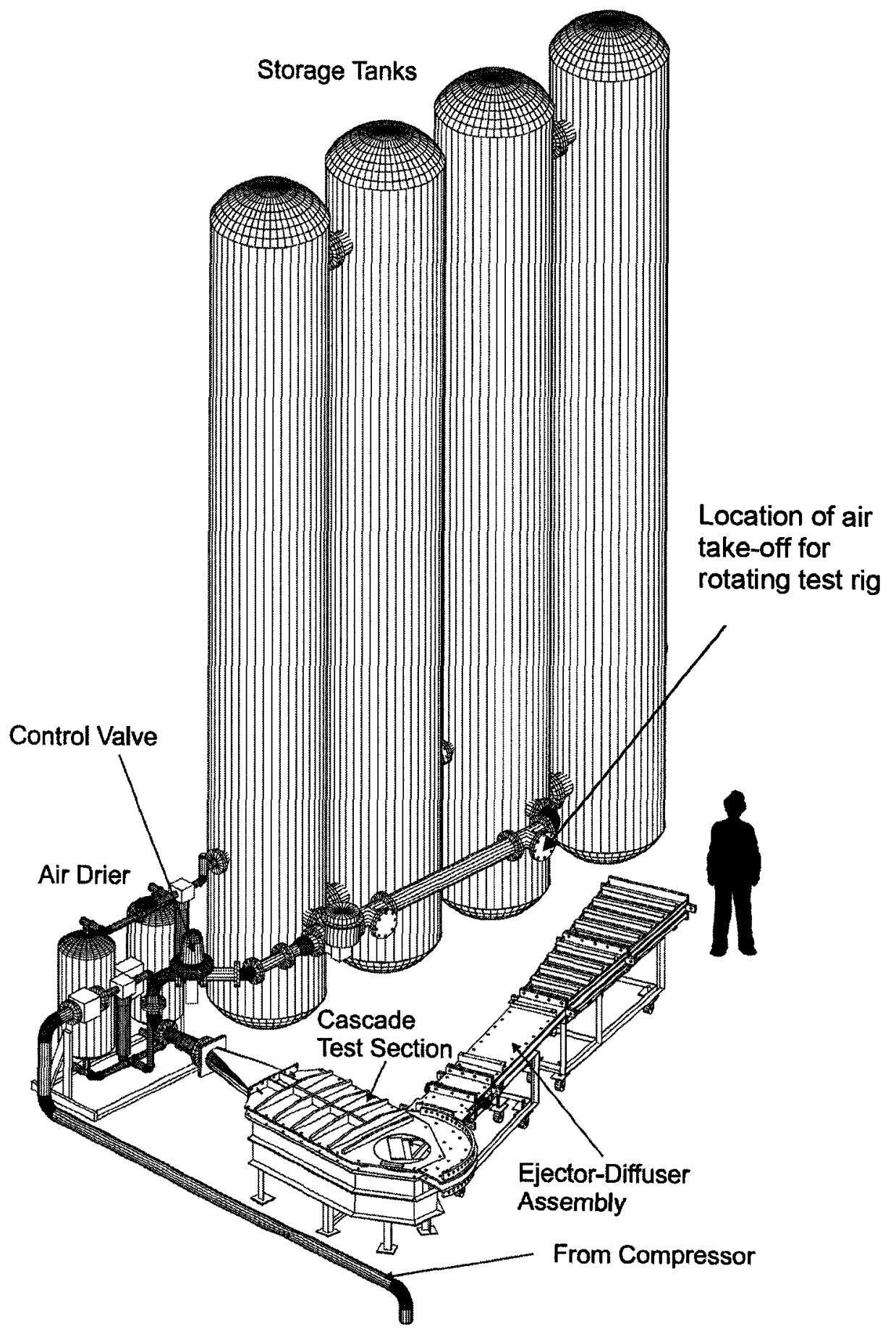

Figure 3.4: High Speed Wind Tunnel Facility (Jeffries, 1994) 
The control system used to control the speed of the turbocharger is shown schematically in Figure 3.5. The blowing pressure from the tanks is measured at the turbine inlet and is controlled by an electro-pneumatic valve which receives its signal from the HSWT control computer. A PID module in the control software allows the blowing pressure and therefore the speed of the compressor to be maintained to within 500 RPM at all running speeds, which are typically from 45,000 to 83,000 RPM. The control software permits the manual adjustment of the blowing pressure during a test run to allow the desired speed to be obtained. For more information on the original HSWT facility and control system see Jeffries (1994). For information regarding the modifications of the control system for use with the rotating test rig see Roberts (2001).

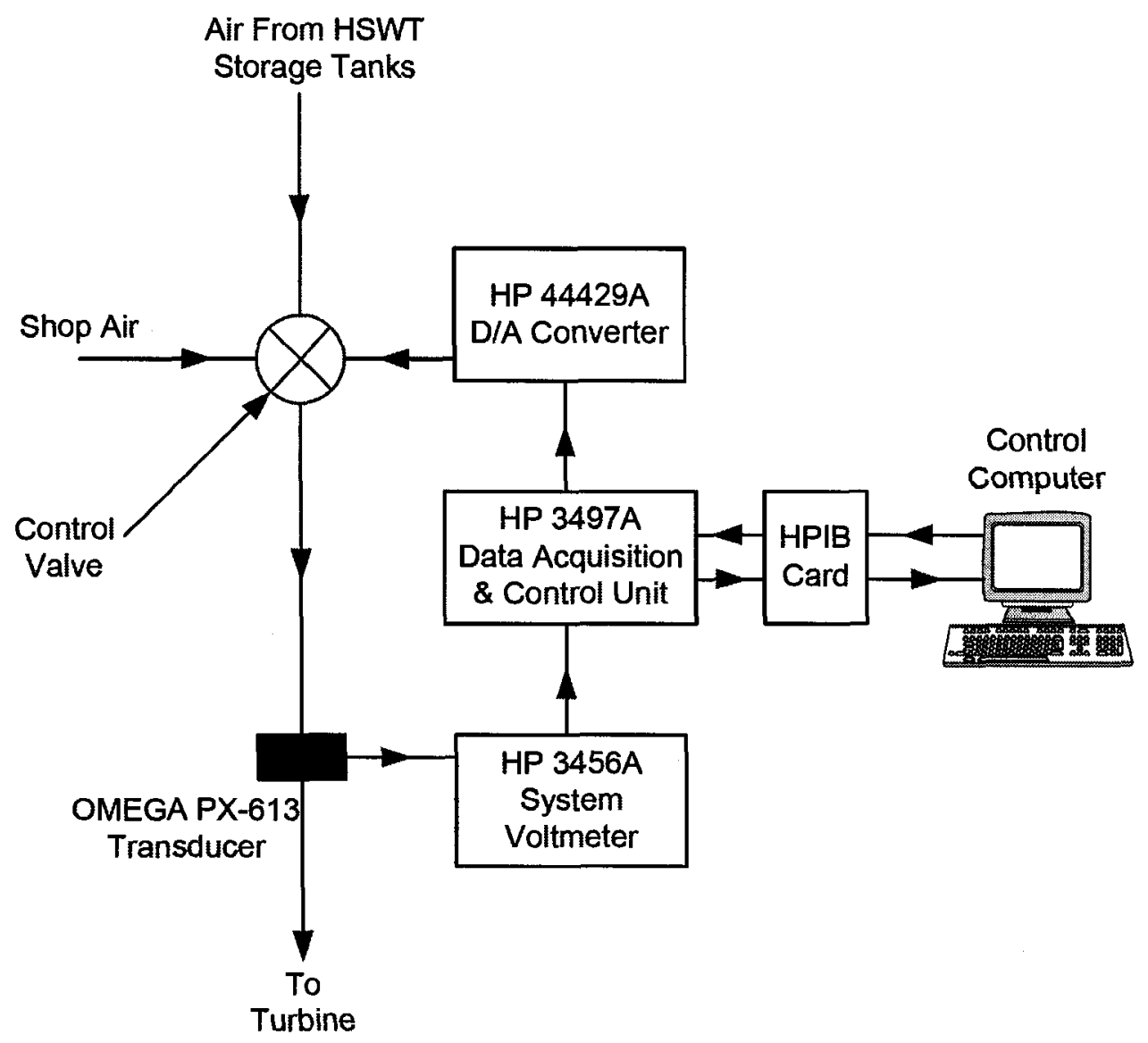

Figure 3.5: Control system flow chart 


\subsection{METHODS OF MEASUREMENT AND DATA REDUCTION}

\subsection{INTRODUCTION}

This chapter describes all instrumentation and data acquisition equipment used in the experimental testing. In addition, the data reduction methods used to determine the compressor performance, are reviewed. This includes an analysis of the uncertainties in the measured flow properties and in the calculated performance parameters. These uncertainties will be discussed in Section 4.7.

The test apparatus was originally instrumented by Roberts such that the overall stage performance parameters of the test compressor could be determined. These parameters are the machine Reynolds number, mass flow parameter, speed parameter, total pressure ratio and isentropic efficiency. The measurements required to determine the overall stage performance are the inlet total temperature and pressure, discharge total temperature and pressure, the flow rate and the shaft speed. The locations of the pressure and temperature measurements, flow meters and the test compressor are in accordance with Section 4.3 of ASME PTC 10. Fully developed and circumferentially uniform flow is assured by having sufficient pipe lengths upstream and downstream of all instrumentation and thus the required pressures and temperatures can be obtained by single point measurements. The instrumentation used for obtaining the overall stage performance data as originally installed by Roberts is described in Section 4.3.

During the course of the present work it was decided to improve upon the capabilities of the rotating test rig. To do so, new instrumentation was added to measure the impeller outlet total pressure and flow angle. In addition, static pressure taps were added which measured the static pressures on the volute and shroud surfaces. The new instrumentation is detailed in Section 4.4. 


\subsection{SENSORS}

\subsubsection{Differential Pressure Transducer}

A Druck PDCR-22 differential pressure transducer was used to measure the pressure difference across the flow meter. It has a nominal $\pm 34.5 \mathrm{kPa}( \pm 5 \mathrm{psi}$ ) range and a $50 \mathrm{mV}$ full scale output at $12 \mathrm{~V}$ supplied power. It has a nominal zero output of $0 \mathrm{mV}$.

The differential pressure transducer was calibrated using a Kulite brand absolute pressure transducer. Until abandoned for safety reasons, a mercury manometer was used in the past as the pressure standard in the HSWT facility. With the unavailability of the mercury manometer, the Kulite transducer was selected because it had demonstrated its linearity and stability for more than ten years in the HSWT facility. Its calibration and reliable operation is well documented in the HSWT in Master's or doctoral theses produced by Jeffries (1994 \& 2000), Islam (1999), Jouini (2000), Corriveau (2005) and Yuan (2006). To verify its continued stable operation the Kulite transducer is taken annually to the laboratories of the National Research Council of Canada for calibration. More recently, a secondary pressure standard has been obtained for the HSWT and will be used for all transducer calibrations in the future.

The calibration curve of the differential pressure transducer is shown in Figure 4.1. The calibration curve is linear and stable, with approximately a $0.4 \mathrm{mV}$ shift of the nominal zero reading over a period of about sixteen months. To remove the corresponding error a zero reading was taken prior to and following each run. The averaged zero reading was then used to correct for the zero shift of the transducer in the data reduction procedure. 


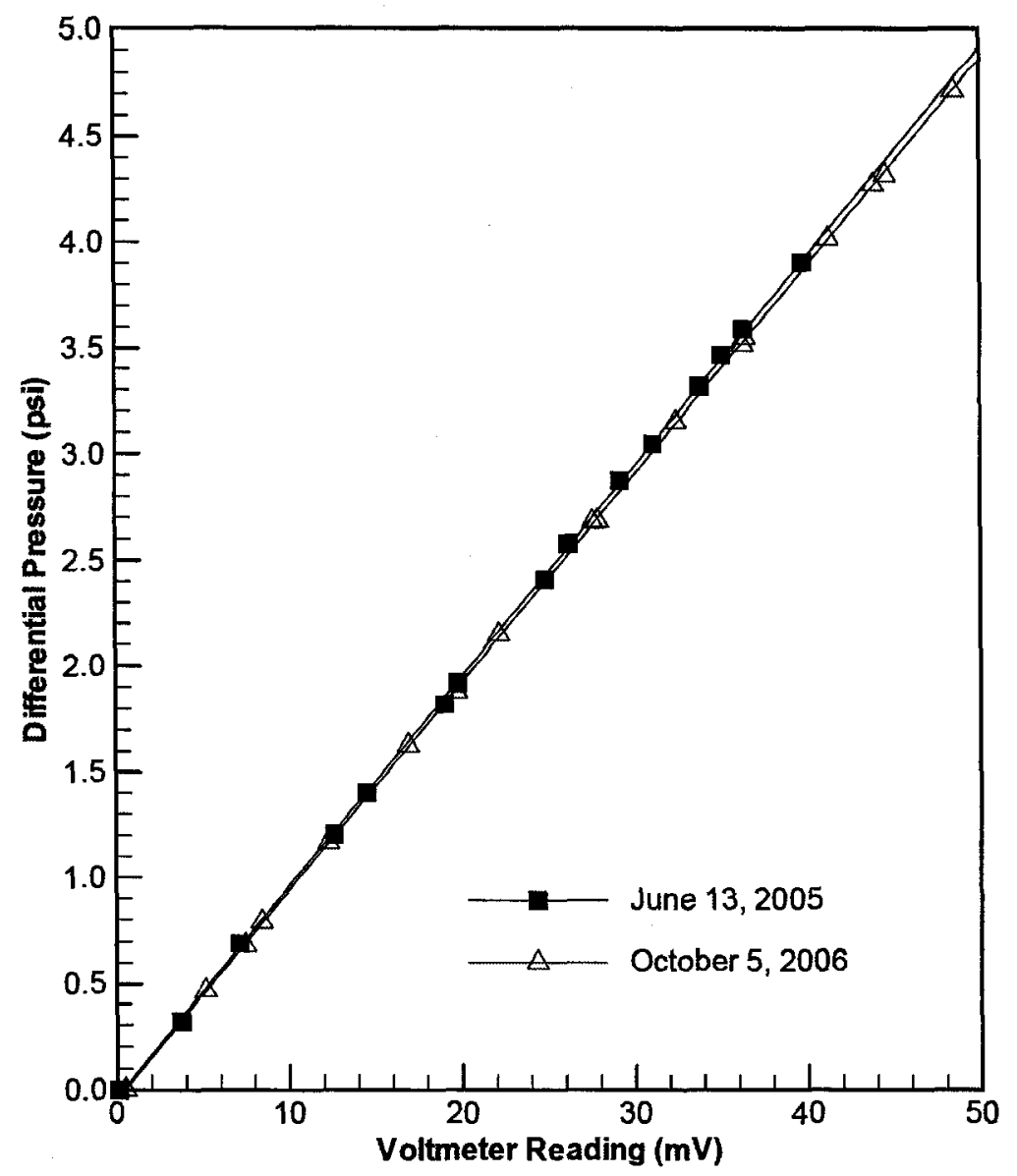

Figure 4.1: Differential pressure transducer calibration curve

\subsubsection{Absolute Pressure Transducer and Scanivalve $\mathbb{B}$}

A Druck PDCR-22 absolute pressure transducer was used to measure all additional pressures by means of a Scanivalve® model SSS-48C. The transducer has a nominal $345 \mathrm{kPa}(50 \mathrm{psi})$ range and a $75 \mathrm{mV}$ full scale output at $12 \mathrm{~V}$ supplied power. This corresponds to a nominal output of approximately $22 \mathrm{mV}$ at atmospheric pressure.

The absolute pressure transducer was also calibrated using the Kulite reference absolute transducer. The calibration was limited by the range of the Kulite transducer and was completed for absolute pressures of $34.5-172 \mathrm{kPa}(5-25 \mathrm{psi})$. The range used 
for calibration was adequate since measured pressures during testing were typically less than $172 \mathrm{kPa}(25 \mathrm{psi})$ and never exceeded $207 \mathrm{kPa}(30 \mathrm{psi})$. The calibration curve is shown in Figure 4.2 and was found to be linear and stable.

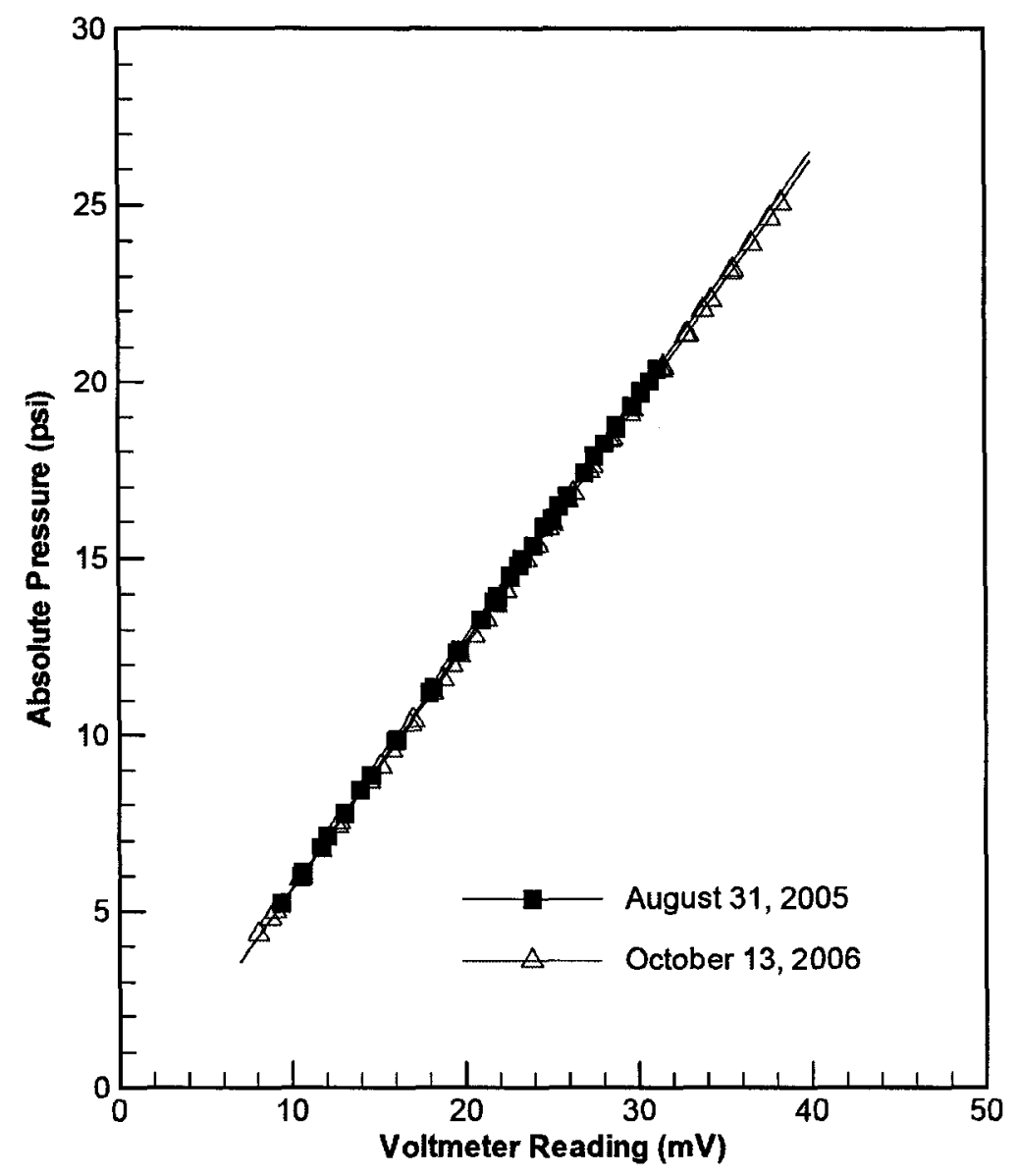

Figure 4.2: Absolute pressure transducer calibration

The absolute pressure transducer was used to measure upwards of 30 separate pressure ports. The Scanivalve $\circledast$ model SSS-48C is capable of sampling 48 pressure ports at scan rates up to 24 ports per second. Since the duration of each test run did not demand such speeds, the scanning rate was configured for approximately 10 ports per second allowing for 200 samples at a frequency of $2000 \mathrm{~Hz}$. In addition, there is a $0.05 \mathrm{~s}$ delay after switching ports on the Scanivalve $\AA$ before the data are collected to allow the 
pressure to settle. The time delay was determined by examining the pneumatic response

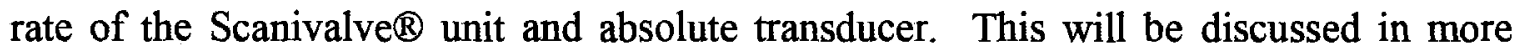
detail in Section 4.5.

\subsubsection{Thermocouples}

Type E thermocouples were selected by Roberts (2001) to measure both the inlet and discharge total temperatures because they provide the highest millivolt output per degree temperature change for the temperatures observed in the experiments. The precision of Type $E$ thermocouples provides highly accurate temperature, and subsequently highly accurate efficiency estimations. The signals from the thermocouples are acquired using a multiplexing thermocouple compensated voltmeter which contains an internal temperature reference junction and outputs the temperature directly in ${ }^{\circ} \mathrm{C}$ using software compensation. The accuracy of the thermocouples was tested by Roberts (2001) using the boiling and freezing point of water. It was found that both probes were accurate to within $0.1^{\circ} \mathrm{C}$.

\subsubsection{Tachometer}

The shaft speed was measured using a Monarch Instruments IRS-5W infrared tachometer installed in the turbine discharge. Using a photodiode and phototransistor the tachometer is able to detect contrasting light and dark surfaces. To maximize contrast a single turbine blade was painted white and all other blades were painted black. When the white blade passes the tachometer, the signal from the photodiode is reflected back to the phototransistor and a $5 \mathrm{~V}$ TTL pulse is sent to the data acquisition system. The tachometer was calibrated by measuring the output frequency when exposed to a fluorescent light. As mentioned previously, the uncertainty of the shaft speed measurement is discussed later in Section 4.7. 


\subsubsection{Relative Humidity}

To obtain accurate values of the gas constant $(R)$ and isentropic exponent $(\gamma)$ of air, the relative humidity was measured using a Mannix PTH-8708 temperature and humidity pen. The humidity measurements are accurate to $\pm 5 \%$. Knowing the ambient pressure and temperature it was possible to determine the mass fraction of water vapour in the air. The fluid properties were then calculated assuming an ideal mixture of dry air and water vapour.

\subsection{Probes PreViously INSTALled by RoBerts}

\subsubsection{Flow Meter}

The inlet flow velocity was measured using an ISA short radius flow nozzle with a diameter ratio (B) of 0.7552 and $39.65 \mathrm{~mm}$ bore. The differential pressure $(\Delta P)$ was measured across the flow meter using static pressure taps located at the specified one pipe diameter upstream of the nozzle and at the nozzle throat. The inlet pipe velocity is calculated from:

$$
V=C_{D} \sqrt{\frac{2 \Delta P}{\rho\left(1-\mathrm{B}^{4}\right)}}
$$

The discharge coefficient $\left(C_{D}\right)$ is calculated for each operating test point using the ISO correlations for ISA short radius nozzles (Urner, 1997).

$$
C_{D}=\frac{\left(1-\mathrm{B}^{4}\left(\frac{P_{2}}{P_{1}}\right)^{\frac{2}{\gamma}}\left(\frac{\gamma}{\gamma-1}\right)\left(1-\left(\frac{P_{2}}{P_{1}}\right)^{\frac{\gamma-1}{\gamma}}\right)\right.}{\left(1-\mathrm{B}^{4}\left(\frac{P_{2}}{P_{1}}\right)^{\frac{2}{\gamma}}\right)\left(1-\frac{P_{2}}{P_{1}}\right)}
$$


The flow meter was manufactured by K-Flo Fluid Meters, Inc. of Montreal to tolerances of $0.0254 \mathrm{~mm}(0.001 \mathrm{in}$.). The uncertainty in the mass flow rate is discussed later.

\subsubsection{Inlet Pressure Probes}

The inlet pressure was measured using the average of four static pressure taps located at $90^{\circ}$ intervals and $61 \mathrm{~cm}(24$ in.) or 8 pipe diameters upstream of the compressor inlet. The $0.457 \mathrm{~mm}(0.018 \mathrm{in}$.) pressure taps are drilled through $3.18 \mathrm{~mm}$ (1/8 in.) MPT brass pipe plugs which are inserted into the pipe. The pipe plugs were ground and polished such that they are flush with the inside pipe surface. The inlet total pressure is required for determining the compressor performance and is calculated from the static pressure, total temperature and average flow velocity in the inlet pipe as determined from the flow meter.

\subsubsection{Inlet Temperature Probe}

The inlet total temperature is measured using a United Sensor T-8-E-12-C Type E thermocouple probe located $30.5 \mathrm{~cm}$ (12 in.) or 4 pipe diameters upstream of the compressor inlet. The tip of the thermocouple probe is shown schematically in Figure 4.3 and consists of a grounded thermocouple junction mounted in a small vented cavity. The probe tip is positioned in the centre of the inlet pipe and aligned with the flow direction.

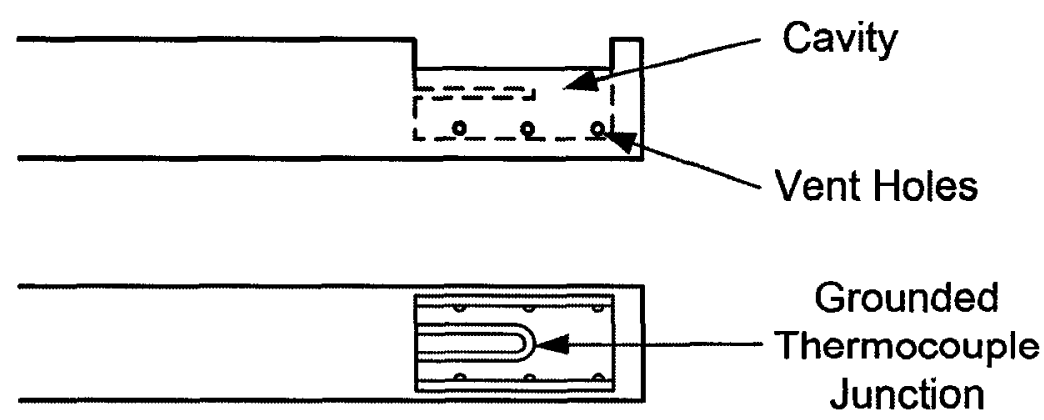

Figure 4.3: Inlet total temperature probe tip 


\subsubsection{Discharge Temperature/Pressure Probe}

The compressor discharge flow conditions are measured using a United Sensor KT-8-E-12-E Type E combination total temperature/total pressure probe. The probe is similar in design to the inlet total temperature probe with the addition of a Kiel probe used for the total pressure measurement. A Kiel probe is insensitive to flow angularity up to about $30^{\circ}$ and should reduce measurement errors due to swirling flow. When originally installed by Roberts (2001) the probe was traversed across the discharge flow and it was found that the temperature and pressure at the pipe centre represented the bulk flow values. The probe position was fixed by Roberts in order to seal the clearance between the probe and the discharge pipe and reduce leakage from the test rig. All discharge measurements were therefore made at the pipe centreline. It is expected that a single large vortex would be generated in the overhung volute of the T04E-50 compressor. Thus the flow angularity near the centreline of the discharge pipe should be within the $30^{\circ}$ range of a Kiel probe. The discharge temperature/pressure probe is shown schematically in Figure 4.4.

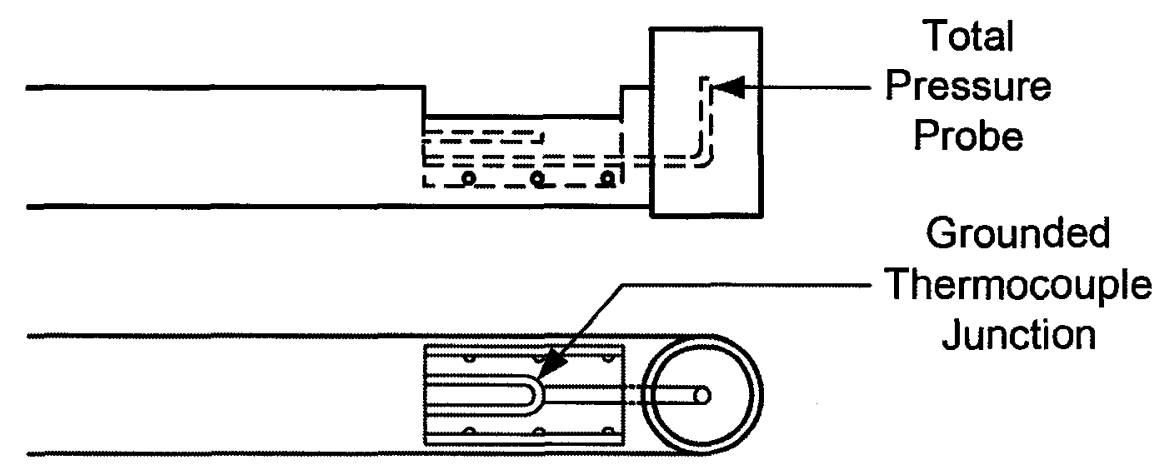

Figure 4.4: Discharge total temperature/total pressure probe tip 


\subsection{Probes Added DURIng Test Rig Modifications}

\subsubsection{Impeller Outlet Total Pressure Probes}

The flow conditions at the impeller outlet were measured using a set of eight total pressure probes manufactured specifically for use in this study. In addition to the probes, collars were also manufactured to fix the probes to the compressor casing. The configuration of the probes and collars as they are installed on the test compressor is shown schematically in Figure 4.5. The geometry of the total pressure probes and collars is shown in Figure 4.6.

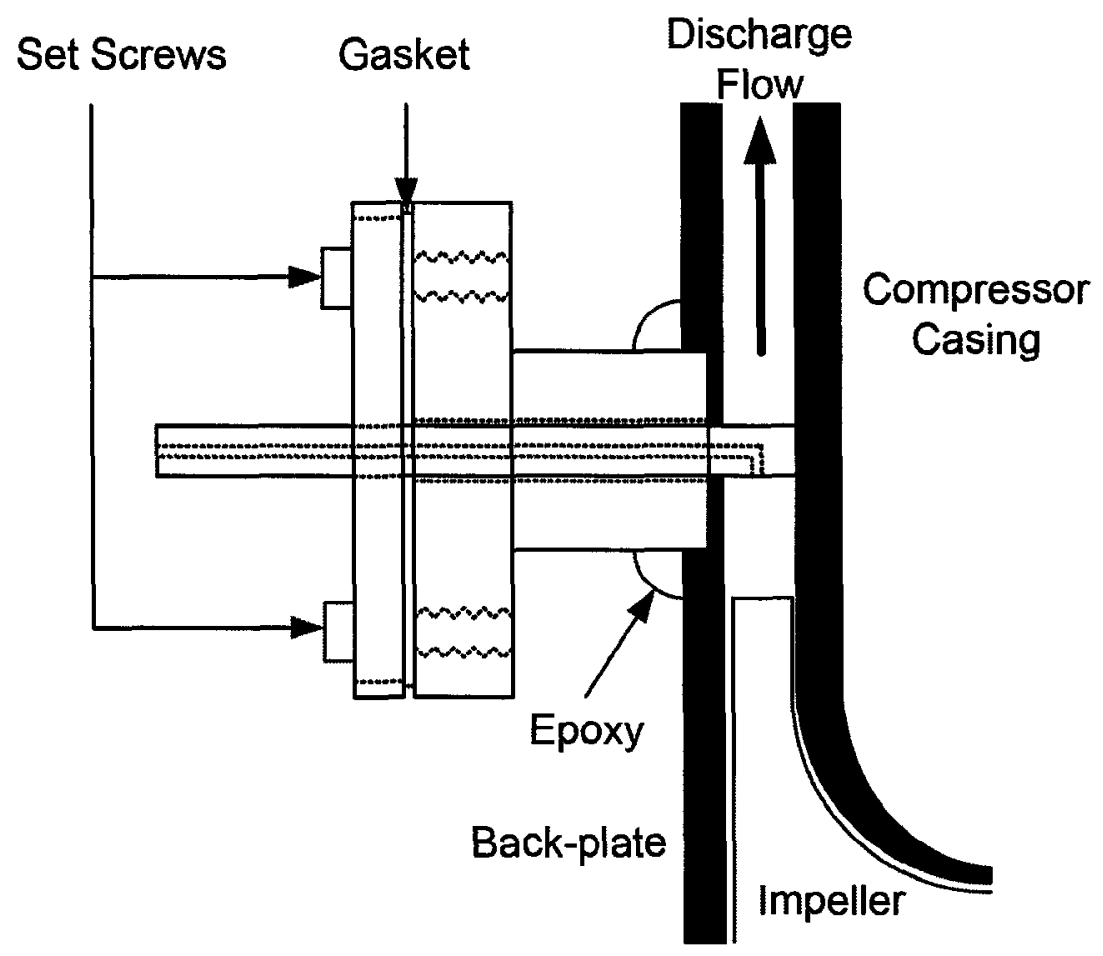

Figure 4.5: Schematic of an assembled impeller outlet total pressure probe 


\section{Total Pressure Probe}

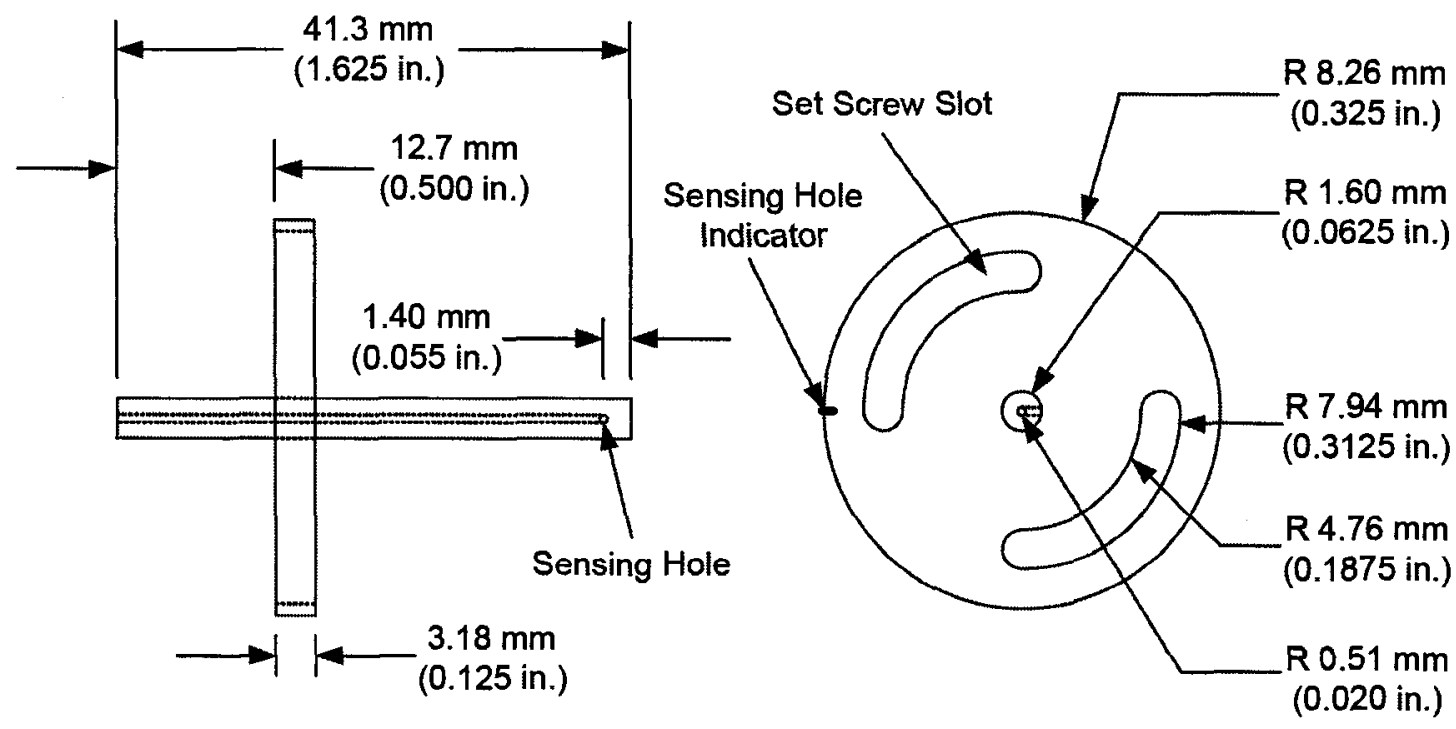

\section{Probe Collar}

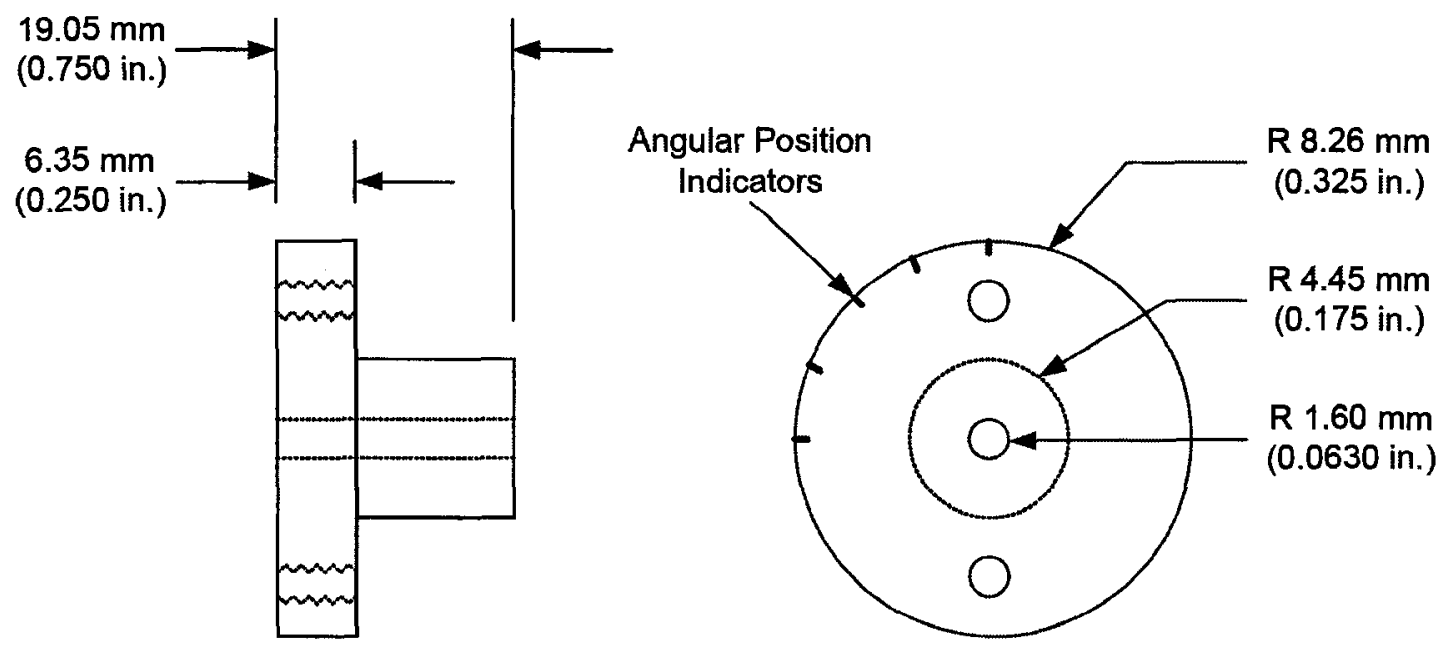

Figure 4.6: Impeller outlet total pressure probe geometry

The probes are installed on the back-plate of the compressor casing using the specifically-designed collars shown. The collar is fixed to the back-plate of the compressor casing using epoxy to reduce leakage. The angular indicating marks have been etched into the larger cylindrical surface of the collar at known angles relative to the 
radial direction of the compressor. A similar etching has been made at $180^{\circ}$ to the sensing hole on the outer cylinder of the total pressure probe. These indicators allow the position of the probe sensing hole to be read when the probe is installed in its collar and through the casing. The probes can then be rotated and the sensing hole aligned at five angles between $0^{\circ}$ and $90^{\circ}$ relative to the radial direction, at $22.5^{\circ}$ increments. For a given test point the probe is held in place by two set screws and a gasket is fitted between the probe and collar to prevent leakage. In total, eight probes are installed circumferentially around the back-plate of the compressor casing at a constant radius of $52 \mathrm{~mm}$. Their locations are shown schematically in Figure 4.7 .

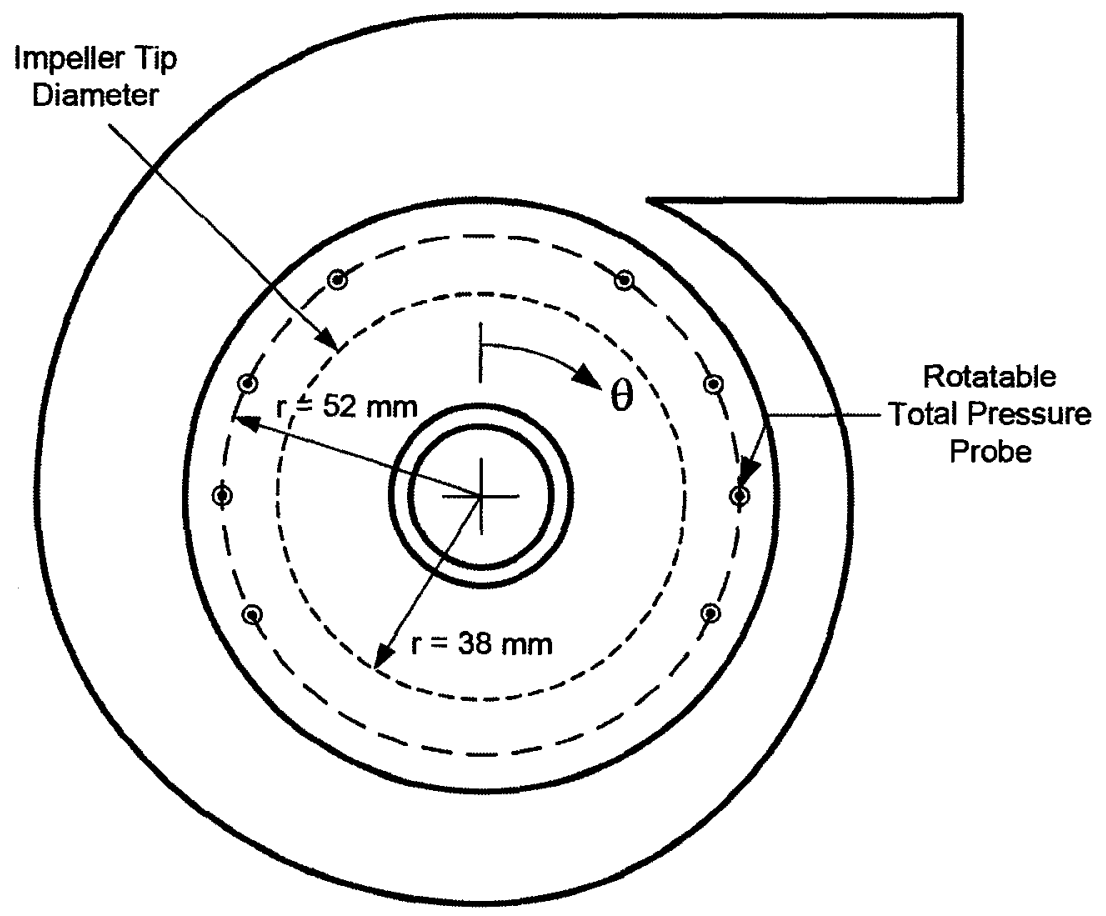

Figure 4.7: Schematic of the impeller outlet total pressure probe locations

\subsubsection{Shroud Surface Static Pressure Taps}

The static pressure variation on the shroud surface was measured using two series of static pressure taps. The locations of the pressure taps are summarized in Table 4.1 and Figure 4.8. The sensing hole of each tap has a diameter of $0.5 \mathrm{~mm}(0.020 \mathrm{in}$.) on the 
inside surface of the shroud. The tap is larger in diameter near the outer surface of the compressor casing such that a $1 \mathrm{~mm}$ (standard $0.040 \mathrm{in}$.) stainless steel tubulation can be inserted and held in place with epoxy.

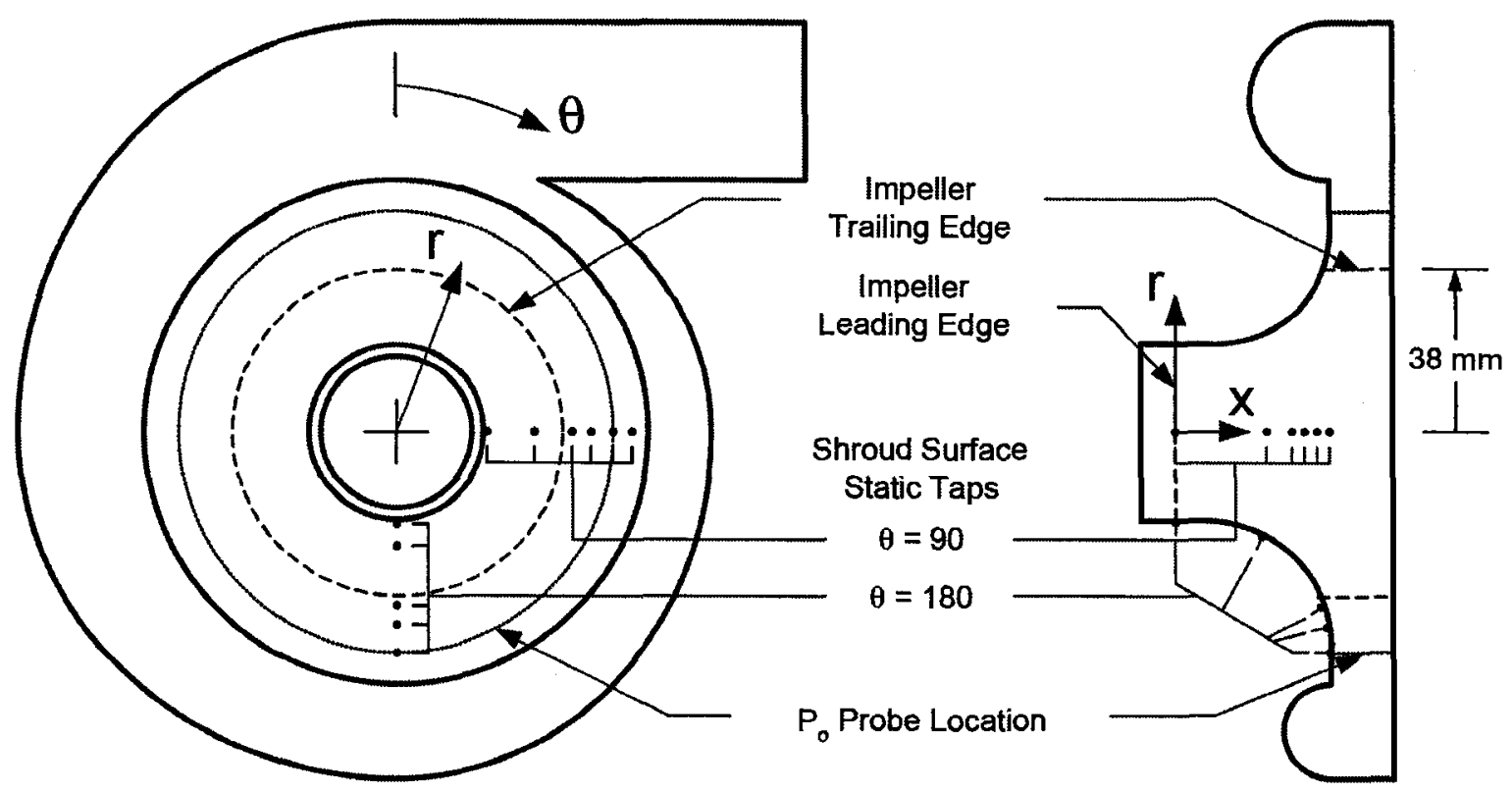

Figure 4.8: Schematic showing the shroud surface static tap locations

\begin{tabular}{|c|c|c|}
\hline & Radial Position, $\mathrm{r}(\mathrm{mm})$ & Axial Position, $\mathrm{x}(\mathrm{mm})$ \\
\hline \multirow{4}{*}{$\begin{array}{c}\text { Circumferential } \\
\text { Position } \\
\theta=90^{\circ}\end{array}$} & 27.2 & 0.0 \\
\cline { 2 - 3 } & 34.3 & 14.7 \\
\cline { 2 - 3 } & 42.3 & 24.5 \\
\cline { 2 - 3 } & 46.6 & 24.5 \\
\cline { 2 - 3 } & 52.1 & 24.5 \\
\hline \multirow{4}{*}{$\begin{array}{c}\text { Circumferential } \\
\text { Position } \\
\theta=180^{\circ}\end{array}$} & 55.9 & 24.5 \\
\cline { 2 - 3 } & 27.2 & 0.0 \\
\cline { 2 - 3 } & 29.5 & 8.4 \\
\cline { 2 - 3 } & 42.3 & 24.5 \\
\cline { 2 - 3 } & 46.6 & 24.5 \\
\hline
\end{tabular}

Table 4.1: Summary of the shroud surface static tap coordinates 


\subsubsection{Volute Static Pressure Taps}

The static pressure variation around the volute was also measured, using two series of static pressure taps. The two series were located at $90^{\circ}$ intervals around the circumference and were aligned in the axial and radial directions. The taps were created similar to the shroud surface taps and their locations are shown schematically in Figure 4.9 .

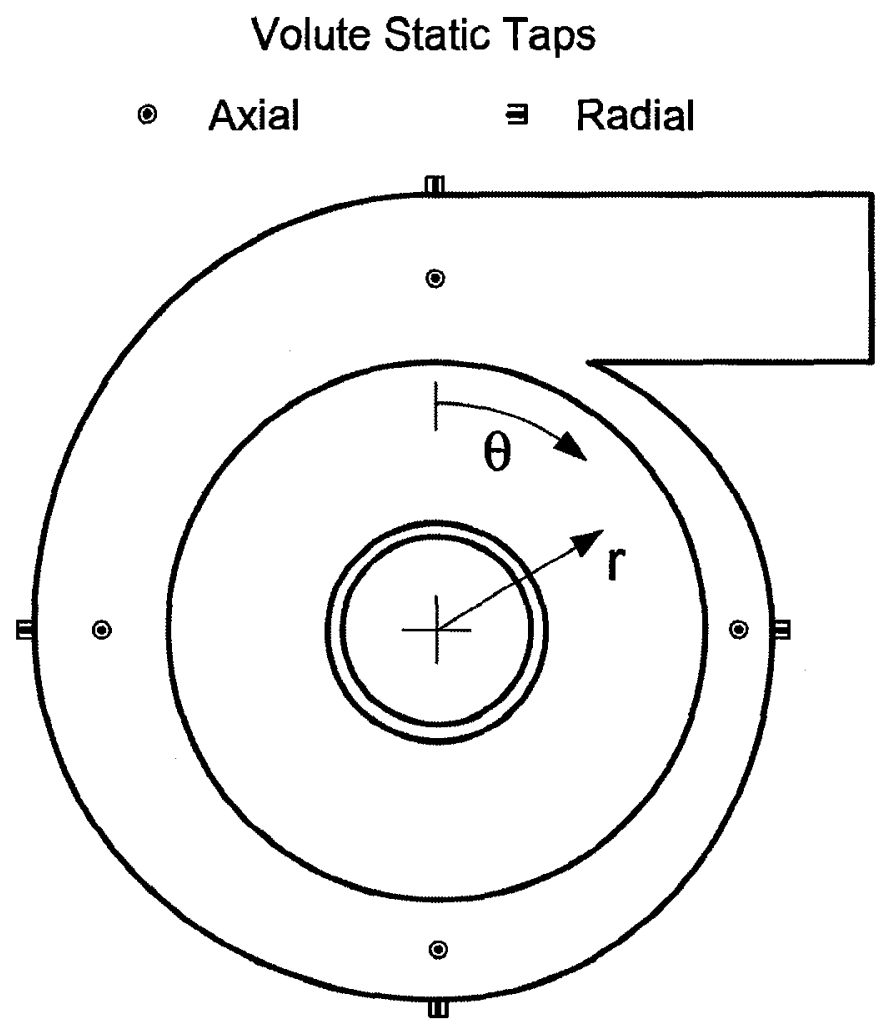

Figure 4.9: Schematic showing the volute static pressure tap locations 


\subsection{DATA ACQUISITION}

\subsubsection{Summary of the Data Acquisition System}

As originally designed by Roberts (2001), data acquisition was performed using an HP 3852A data acquisition and control unit that was also used with the main HSWT facility. A flow diagram of the instrumentation and data acquisition system as configured fro the rotating test rig is shown in Figure 4.10.

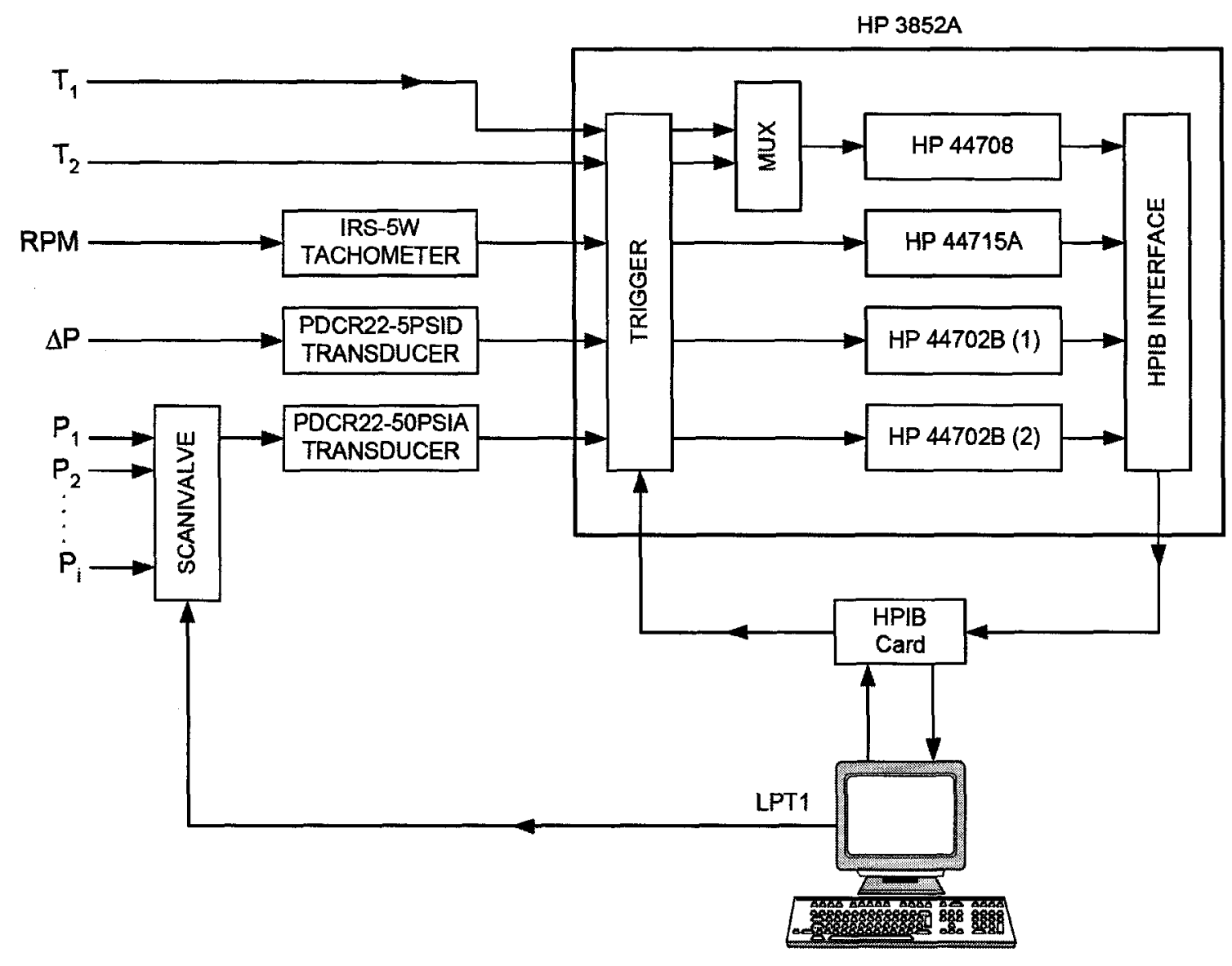

Figure 4.10: Signal flow chart of the instrumentation and data acquisition system 
The data acquisition software was originally designed for the HSWT by Jeffries (1994). For a more detailed review of the original data acquisition system and software the reader is referred to the work of Jeffries (1994). To accommodate the additional pressure, temperature and shaft speed measurements of the rotating test rig, minor additions were made to the original software. The additions were made by Roberts (2001) and are described in his thesis. The current study required a larger number of pressure measurements to be taken than originally obtained in Roberts (2001). Since the Scanivalve@ unit is capable of handling additional pressure measurements modifications to the data acquisition software were not required. Nevertheless, the data acquisition software was reviewed and slightly modified to generate more appropriate output files for the current measurements.

\subsubsection{Pressure and Temperature Measurements}

As mentioned previously, the rotating test rig utilizes two pressure transducers for all pressure measurements made. A PDCR- $22 \pm 34.5 \mathrm{kPa}( \pm 5 \mathrm{psi}$ ) differential transducer is used to measure the differential pressure across the flow meter and a PDCR-22 345 $\mathrm{kPa}(50 \mathrm{psi})$ absolute transducer is used for all other pressure measurements. The voltage signal from each transducer is sent to one of the two HP 44702B 13-Bit high speed voltmeter cards installed in the HP 3852 data acquisition mainframe. The range of the voltmeter used with the absolute pressure transducer was set to $320 \mathrm{mV}$. This corresponds to a resolution of $78.13 \mu \mathrm{V}$ or 0.05 psia. The voltmeter range for the differential pressure transducer was set to $40 \mathrm{mV}$ which corresponds to a resolution of $9.766 \mu \mathrm{V}$ or $0.001 \mathrm{psid}$. To obtain a pressure measurement, 200 samples are taken at $2000 \mathrm{~Hz}$. The 200 samples are then averaged to give the mean value for the measured

pressure. The number of samples and sample frequency were originally selected by Roberts (2001) to match those used in the HSWT and it has been found that these values produce repeatable mean values for the quantities measured in both the rotating test rig and HSWT. Sample outputs of the absolute transducer are shown in Figure 4.11. Included in the figure is the running average for the same data sets. The running average 
is the average of the samples up to the point at which a sample is taken. The samples within the $0.05 \mathrm{~s}$ delay are shown to illustrate the pneumatic response of the system after switching Scanivalve $\otimes$ ports and are not actually collected during testing. As seen the running average of the collected data is stable after 200 samples.

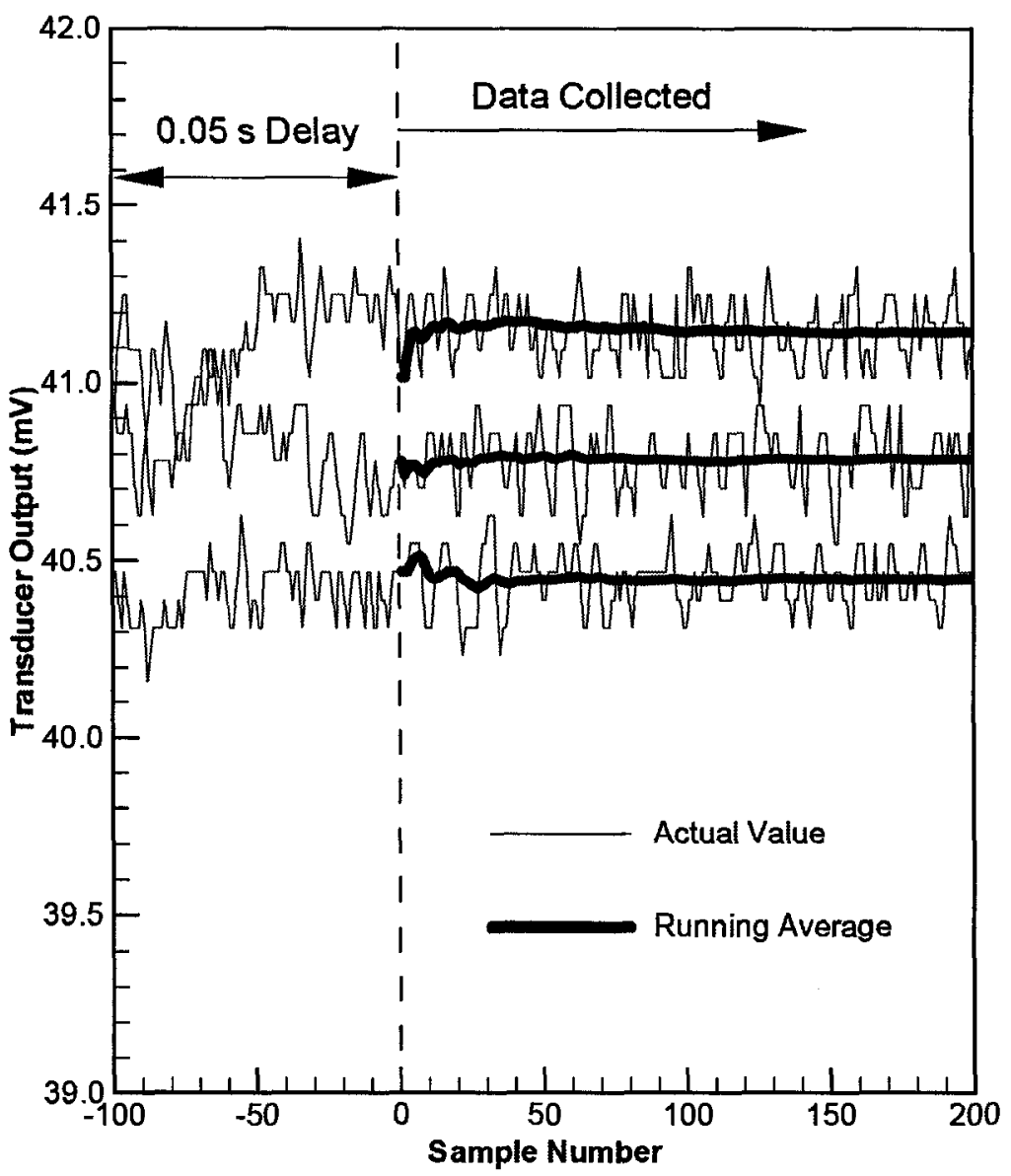

Figure 4.11: Sample transducer output

The temperature measurements are made using an HP 44708 20-Channel Multiplexing Thermocouple Compensated Voltmeter. The voltmeter has 13-bit resolution and outputs the temperature directly with a resolution of $0.1{ }^{\circ} \mathrm{C}$. Similar to the pressure measurements, a temperature measurement is the average of 200 samples at $2000 \mathrm{~Hz}$. 


\subsubsection{Shaft Speed Measurement}

The frequency of the TTL pulse from the tachometer is measured using an HP $44715200 \mathrm{kHz} 5$-Channel Counter/Totalizer. The shaft speed is determined by summing the number of tachometer pulses in a sampling time of $1 \mathrm{~s}$. The resolution of the frequency counter is therefore $1 \mathrm{~Hz}$ or $60 \mathrm{RPM}$. The shaft speed for a given test point is determined by averaging five speed measurements of $1 \mathrm{~s}$ duration. The variation in these five measurements was used to estimate the uncertainty in the measured values of shaft speed and is discussed later.

\subsection{DAta REDUCTION}

\subsubsection{Properties of Air}

The maximum temperature variation measured between all test points was less than $100^{\circ} \mathrm{C}$. The relative humidity varied depending on the current atmospheric conditions of the test day. It was therefore necessary to account for the variations in relative humidity due to its influence on the mass flow parameter and the efficiency. The humidity affects the mass flow parameter by means of the density and affects the efficiency by means of the isentropic exponent.

The properties of moist air were modelled as an ideal mixture of dry air and water vapour. Curve fit equations from Irvine (1984) were used to determine the fluid properties of each constituent. The fluid properties of the mixture were then determined from the mass-averaged properties for dry air and water vapour at the average temperature between the inlet and discharge of the compressor. 
First the specific humidity $(\omega)$ is found from the relative humidity $(R H)$.

$$
\omega=0.62188 R H \frac{P_{\text {sat }}}{P_{\text {amb }}-0.37812 R H^{*} P_{\text {sat }}}
$$

where $P_{\text {sat }}$ is the saturation pressure of water vapour at the ambient temperature and $P_{a m b}$ is the ambient pressure. The saturation pressure of water vapour $\left(P_{\text {sat }}\right)$ expressed in $\mathrm{kPa}$ is found from Irvine (1984) when the temperature $(T)$ is specified in Kelvin.

$$
P_{\text {sat }}=e^{\left(\sum_{n=0}^{n=9} a_{n} T^{n}\right)}-\left(\frac{b}{T-c}\right)
$$

The specific heats at constant pressure of dry air and water vapour are also found from Irvine (1984).

$$
\begin{gathered}
c_{P a i r}=\sum_{n=0}^{n=4} a_{n} T^{n} \\
c_{P_{w}}=237.6 \times 10^{-9} T^{2}-97.9 \times 10^{-6} T+1.847
\end{gathered}
$$

The specific heat is given in $\mathrm{kJ} / \mathrm{kgK}$ when $T$ is in Kelvin. The coefficients for Equations 4.4 and 4.5 can be found in Appendix A. The mixture properties are then determined from the mass-averaged properties of dry air and water vapour as follows:

$$
\begin{aligned}
& c_{P}=m_{\text {air }} c_{P a i r}+m_{w} c_{P w}=(1-\omega) c_{P a i r}+\omega c_{P_{w}} \\
& R=m_{\text {air }} R_{\text {air }}+m_{w} R_{w}=R_{U}\left[\frac{(1-\omega)}{M w_{\text {air }}}+\frac{\omega}{M w_{w}}\right]
\end{aligned}
$$


$R_{U}=8.314 \mathrm{~kJ} / \mathrm{kgK}$ is the universal gas constant and $m$ and $M w$ are the mass fraction and the molecular weight of each constituent respectively. The isentropic exponent $(\gamma)$ is:

$$
\gamma=\frac{c_{P}}{c_{V}}=\frac{c_{P}}{c_{P}-R}
$$

\subsubsection{Test Parameters}

\section{Mass Flow Parameter:}

In order to determine the mass flow parameter the mass flow rate through the compressor must first be known. The mass flow rate $\dot{m}$ is obtained using the flow velocity $V$, area $A$ and density $\rho$, all at the throat of the flow nozzle.

$$
\dot{m}=\rho A V
$$

The flow velocity at the nozzle throat was calculated using Equations 4.1 and 4.2 from Section 4.3.1.

$$
\begin{gathered}
V=C_{D} \sqrt{\frac{2 \Delta P}{\rho\left(1-\mathrm{B}^{4}\right)}} \\
C_{D}=\frac{\left(1-\mathrm{B}^{4}\left(\frac{P_{2}}{P_{1}}\right)^{\frac{2}{\gamma}}\left(\frac{\gamma}{\gamma-1}\right)\left(1-\left(\frac{P_{2}}{P_{1}}\right)^{\frac{\gamma-1}{\gamma}}\right)\right.}{\left(1-\mathrm{B}^{4}\left(\frac{P_{2}}{P_{1}}\right)^{\frac{2}{\gamma}}\right)\left(1-\frac{P_{2}}{P_{1}}\right)}
\end{gathered}
$$


The density at the nozzle throat was calculated from the ideal gas law.

$$
\rho=\frac{P}{R T}
$$

Because the static density is required in the flow velocity calculation, the static pressure at the nozzle throat and the inlet static temperature were needed. The nozzle throat static pressure was measured directly by the absolute transducer; however, the total temperature was measured at the inlet. The static temperature $(T)$ can be found from the total temperature $\left(T_{0}\right)$ using the isentropic relationship.

$$
T=T_{0}-\frac{V^{2}}{2 c_{P}}
$$

Since the static temperature is also a function of the flow velocity an iterative process was required. The mass flow parameter was then calculated from Equation 2.13.

$$
\Pi_{m}=\frac{\dot{m} \sqrt{T_{01}}}{P_{01}}\left(\frac{1}{D^{2}} \sqrt{\frac{R}{\gamma}}\right)
$$

\section{Speed Parameter:}

The speed parameter was calculated directly from Eqn. 2.14 using the fluid properties and total temperature at the compressor inlet. As is common practice, the rotational speed $(N)$ is specified in RPM and therefore the speed parameter is not truly non-dimensional.

$$
\Pi_{N}=\frac{N}{\sqrt{T_{01}}}\left(\frac{D}{\sqrt{\mathscr{R}}}\right)
$$




\section{Machine Revnolds Number:}

The machine Reynolds number $\left(R e_{b 2}\right)$ is based on the exit vane height $\left(b_{2}\right)$ and was calculated from Eqn. 2.15.

$$
R e_{b 2}=\frac{\rho_{01} U_{2} b_{2}}{\mu}
$$

The stagnation density $\left(\rho_{01}\right)$ is determined from the measured inlet total temperature and total pressure using the ideal gas law (Equation 4.10). The blade tip speed $\left(U_{2}\right)$ is determined from the rotational speed ( $N$ in RPM) and impeller tip diameter $\left(D_{2}\right)$ from:

$$
U_{2}=\frac{\pi N D_{2}}{60}
$$

The viscosity $(\mu)$ was taken to be equal to $1.846 \times 10^{-5} \mathrm{Ns} / \mathrm{m}^{2}$, the value for air at $300 \mathrm{~K}$.

\section{Impeller Exit Total Pressure and Flow Angle:}

The new total pressure probes used to measure the flow conditions at the impeller outlet are detailed in Section 4.4.1. In total eight new probes were installed at various circumferential positions around the impeller outlet. The sensing hole of the new probes is located on the cylindrical surface of the probe stem. Since the probes can be rotated, the sensing hole can be aligned at multiple angles relative to the flow. For a given operating point, the probes are fixed to a known angle relative to the radial axis of the impeller. Measurements are repeated for five probe angles for the same operating condition. A second-order polynomial is fitted to the data and the peak of the curve is used to estimate the total pressure. The flow angle is then taken as the probe angle at which the curve is a maximum.

Figure 4.12 shows a sample of the measured pressures for various probe angles for a particular operating point. As seen the measured pressure varies with probe angle 
for each probe. In addition, the curve fits yield differing maximum pressures for different probes. Thus there appears to be circumferential variations in the flow at the impeller exit. The results obtained using the probe measurements are discussed in Section 7.3.3.

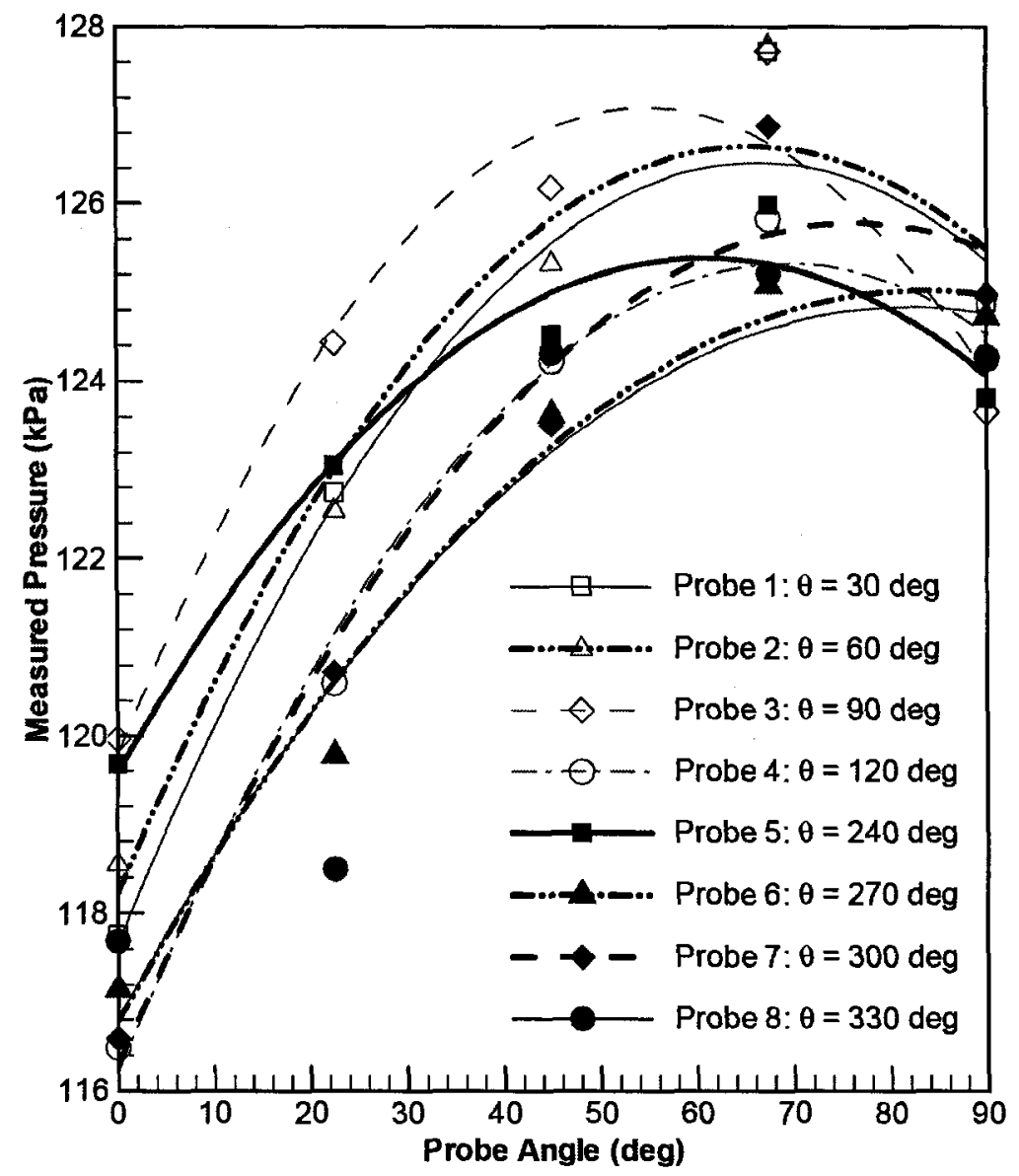

Figure 4.12: Sample measurements and curve fits obtained from the rotatable total pressure probes

It was desired to obtain averaged total pressures and flow angles for the impeller outlet flow. The eight individual pressure measurements were therefore averaged at each probe angle. Since the probes are not evenly spaced around the circumference of the impeller outlet, a trapezoidal area-averaging technique was used to average the data. A 
second-order polynomial was then fitted to the averaged data and the total pressure at the impeller exit was estimated as the peak of this curve fit.

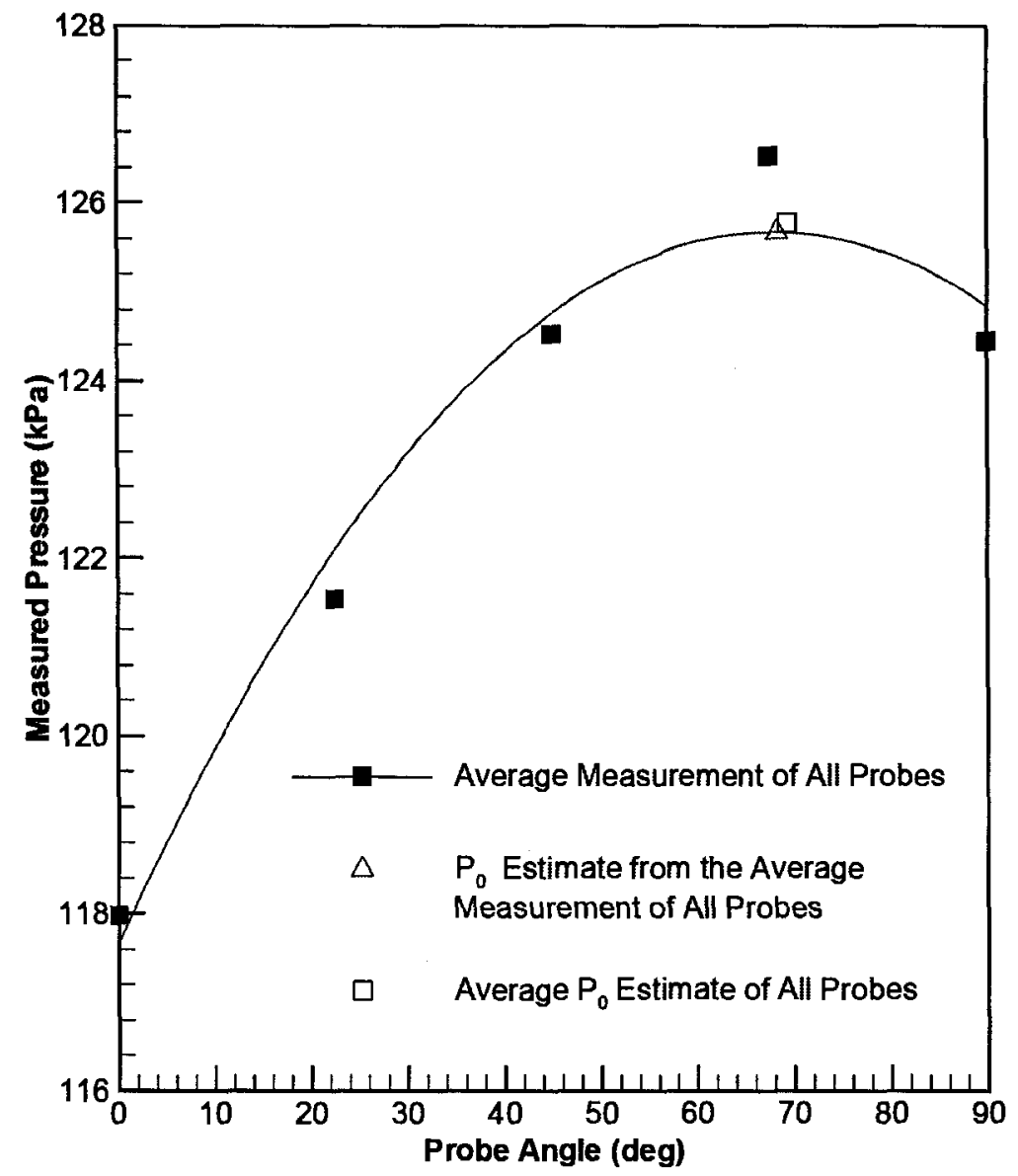

Figure 4.13: Averaged total pressure estimates from the sample measurements of the rotatable total pressure probes

Figure 4.13 shows the averaged pressures measured at various probe angles and the corresponding curve fit. The total pressure and flow angle estimate using the curve fit of the averaged data is shown. An alternative would be to determine the total pressure and flow angle for each probe and then area-average these values circumferentially. The corresponding values are shown on Figure 4.13 using open symbols. It would have been more appropriate to average the components of velocity at each probe location to 
determine the averaged flow angle. However, insufficient information was available at the impeller exit to determine the velocities and thus the averaged flow angle could only be estimated using the flow angle at each probe.

As seen in Figure 4.13, the two estimates of total pressure and flow angle are nearly identical. The experimental performance characteristics of the impeller, presented in Section 7.3.2, were therefore obtained by area-averaging the measured pressures before generating the curve fit and estimating the total pressure and flow angle. The results shown in Figure 4.12 and 4.13 are for a representative operating point and are typical of all operating points for the data set.

\section{Total Pressure Ratio:}

The stage total pressure ratio was calculated from the absolute pressures measured in the inlet and discharge pipes.

$$
P R=\frac{P_{02}}{P_{01}}
$$

The impeller total pressure ratio was calculated using the average total pressure estimate from the rotatable total pressure probes as the outlet condition $\left(P_{02}\right)$.

\section{Isentropic Efficiency:}

The isentropic efficiency of the stage and the impeller were calculated from Equation 2.19 using their respective pressure ratios, and the total temperature measurements made in the inlet and discharge pipes.

$$
\eta=\frac{\left(\frac{P_{02}}{P_{01}}\right)^{\frac{\gamma-1}{\gamma}}-1}{\frac{T_{02}}{T_{01}}-1}
$$




\subsubsection{Corrections to Measured Data}

\subsubsection{Corrections Due to Deviations in Test Speed Parameter}

By manually adjusting the blowing pressure it was possible to match the shaft speed from run to run to within about 500 RPM of the desired speed. This variation corresponds to approximately $1 \%$ of the desired speed at the lowest rotational speed of 46,000 RPM. At higher rotational speeds it was found that the desired speed could be more closely matched and typical variations of the test speed were less than $0.5 \%$ of the specified speed. Nevertheless, minor corrections are made to the pressure ratio to account for differences in the specified and actual shaft speeds and the formulation is taken from Roberts (2001).

The pressure ratio is corrected by assuming that the pressure rise in the compressor is proportional to the square of the rotational speed, then

$$
\left[\frac{1}{N^{2}}\left(\frac{P_{02}}{P_{01}}-1\right)\right]_{t}=\left[\frac{1}{N^{2}}\left(\frac{P_{02}}{P_{01}}-1\right)\right]_{s p}
$$

where the subscripts $t$ and $s p$ represent the conditions at the actual test point and at the desired speed respectively. The corrected pressure ratio at the specified rotational speed is therefore:

$$
\left(\frac{P_{02}}{P_{01}}\right)_{s p}=1+\left[\left(\frac{P_{02}}{P_{01}}\right)_{t}-1\right]\left(\frac{N_{s p}}{N_{t}}\right)^{2}
$$

Equation 4.13 can only be applied to correct for small differences in the shaft speed and should not be used to predict the pressure ratio for a different speed line. 


\subsubsection{Reynolds Number Correction}

The measured performance was corrected for differences in the Reynolds number, such that the experimental data could be compared to the data collected by Roberts (2001) and the data provided by the manufacturer. As in Roberts (2001), the Reynolds number correction was performed using the method of Strub et al. (1987). This method was selected because it has been validated over a wide range of Reynolds numbers, including the particularly low values $\left(R e_{b 2}<55,000\right)$ seen in the experiments. The method of Strub et al. provides corrections for the efficiency, pressure ratio and mass flow parameter.

\section{Efficiency Correction:}

The Reynolds number correction for efficiency is based on an estimated friction factor $\lambda$ in the impeller and takes the form:

$$
\frac{1-\eta_{s p}}{1-\eta_{t}}=\frac{0.3+0.7 \frac{\lambda_{s p}}{\lambda_{c r}}}{0.3+0.7 \frac{\lambda_{t}}{\lambda_{c r}}}
$$

The friction factor at the critical Reynolds number $\lambda_{\text {cr }}$ is calculated from the surface roughness $e$ and the exit vane height of the impeller $b_{2}$ using

$$
\lambda_{c r}=1.74-2 \log _{10}\left(2 \frac{e}{b_{2}}\right)
$$

Strub et al. recommend using the equations from Colebrook (1939) for fully developed circular pipe flow to estimate the friction factor at the test and specified conditions. However, applying the Colebrook equation requires iteration and instead an explicit formulation from Haaland (1983) was used: 


$$
\frac{1}{\sqrt{\lambda}}=-\frac{1.8}{n} \log _{10}\left[\left(\frac{6.9}{R e_{D_{H}}}\right)^{n}+\left(\frac{e}{3.75 D_{H}}\right)^{1.11 n}\right]
$$

As stated by Strub et al., an appropriate mean hydraulic diameter $D_{H}$ for an impeller is $2^{*} b_{2}$ and the mean flow in a compressor stage is approximately $U_{2} / 2$. The Reynolds number based on the hydraulic diameter $R e_{D_{H}}$ is therefore equal to the machine Reynolds number $R e_{b 2}$. In the range of Reynolds numbers seen experimentally, a value of $n=2.75$ provided reasonable agreement with the Colebrook equations.

\section{Pressure Ratio Correction:}

Strub et al. argue that near the best efficiency point approximately half of the increase in efficiency due to an increase in Reynolds number will contribute to an increase in the pressure ratio. The pressure ratio correction is then:

$$
\frac{P R_{s p}-1}{P R_{t}-1}=0.5+0.5 \frac{\eta_{s p}}{\eta_{t}}
$$

\section{Mass Flow Parameter Correction:}

Strub et al. note that the pressure ratio versus mass flow characteristic at a given point is approximately parabolic. In addition, the authors note that the change in pressure rise and mass flow with increasing Reynolds number resembles the change due to a small increase in speed. The correction of the mass flow parameter is therefore of the following form:

$$
\frac{\Pi_{m, s p}}{\Pi_{m, t}}=\sqrt{\frac{P R_{s p}}{P R_{t}}}
$$


where

$$
\Pi_{m}=\frac{\dot{m} \sqrt{T_{01}}}{P_{01}}\left(\frac{1}{D^{2}} \sqrt{\frac{R}{\gamma}}\right)
$$

The variation of machine Reynolds number at a given shaft speed never exceeded $10 \%$ and in general was less than $5 \%$. The resulting efficiency correction for most test points was therefore less than $0.5 \%$. The corrections to the pressure ratio and mass flow parameter were typically less than $0.5 \%$ and $0.2 \%$ of their respectively measured value.

\subsection{Assessment of ERRoRs in Test PaRAmeters}

\subsubsection{Uncertainties in Measured Test Quantities}

The experimental methods used here are nearly identical to those of Roberts (2001), with the exception of the new instrumentation added during the test rig modifications. Therefore the measurement uncertainties for the existing instrumentation are the same as those determined by Roberts (2001). Roberts divided the contributions to the uncertainties of measured quantities into five categories. The uncertainties have been reviewed and re-grouped into four categories as follows:

1. Single Sample Error: This represents the accuracy and resolution of the sensor in taking a single sample, and also includes the uncertainty in the sensor calibration.

2. Measurement Scatter: This represents the scatter in a test measurement, which consists of 200 samples.

3. Fluctuations of measured test quantities during a test: During a given run, small fluctuations in the operating point were caused by fluctuations of the turbine blowing pressure. 
4. Uncertainties in the Apparatus: This represents any systematic errors resulting from the imperfections of the test apparatus. These may include errors due to probe misalignment, pressure tap imperfections, leakage from the test circuit and heat transfer through the insulated components.

The typical scatter of a given test measurement for each sensor, as noted by Roberts (2001), is shown in Table 4.2. The values quoted represented the majority of test cases $(>80 \%)$.

\begin{tabular}{|c|c|c|c|c|}
\hline Measurement & Instrument & $\begin{array}{l}\text { Sample } \\
\text { Error }\end{array}$ & $\begin{array}{c}\text { Sample } \\
\text { Resolution }\end{array}$ & $\begin{array}{c}\text { Typical Scatter } \\
\text { of Single } \\
\text { Measurement }\end{array}$ \\
\hline $\begin{array}{l}\text { Flow meter } \\
\text { Differential } \\
\text { Pressure }\end{array}$ & $\begin{array}{l}\text { Druck PDCR22 } \\
\text { Differential } \\
\text { Pressure } \\
\text { Transducer }\end{array}$ & $0.02 \% \mathrm{FS}$ & $\begin{array}{c}9.766 \mu \mathrm{V} \\
0.00674 \mathrm{kPa} \\
0.000977 \mathrm{psi}\end{array}$ & $\begin{array}{c}0.1 \% \text { of Measured } \\
\text { Differential Pressure }\end{array}$ \\
\hline $\begin{array}{l}\text { Inlet and } \\
\text { Discharge } \\
\text { Absolute } \\
\text { Pressure }\end{array}$ & $\begin{array}{l}\text { Druck PDCR22 } \\
\text { Absolute Pressure } \\
\text { Transducer }\end{array}$ & $0.1 \% \mathrm{FS}$ & $\begin{array}{c}78.13 \mu \mathrm{V} \\
0.359 \mathrm{kPa} \\
0.0521 \mathrm{psi}\end{array}$ & $\begin{array}{l}0.5 \% \text { of Measured } \\
\text { Absolute Pressure }\end{array}$ \\
\hline $\begin{array}{l}\text { Inlet and } \\
\text { Discharge } \\
\text { Temperature }\end{array}$ & $\begin{array}{l}\text { Type-E Grounded } \\
\text { Thermocouple } \\
\text { Junction }\end{array}$ & $0.1^{\circ} \mathrm{C}$ & $0.01^{\circ} \mathrm{C}$ & $\begin{array}{c}0.25 \% \text { of Measured } \\
\text { Absolute } \\
\text { Temperature }\end{array}$ \\
\hline Shaft Speed & $\begin{array}{l}\text { United Sensor } \\
\text { IRS-5W Infrared } \\
\text { Tachometer }\end{array}$ & $1 \mathrm{~Hz}$ & $\begin{array}{c}1 \mathrm{~Hz} \\
60 \mathrm{RPM}\end{array}$ & $\begin{array}{c}0.5 \% \text { of Measured } \\
\text { RPM }\end{array}$ \\
\hline
\end{tabular}

Table 4.2: Typical scatter in single test point measurements 
Before the data were collected the compressor was run at a constant speed until steady-state conditions were reached. This typically took less than 5 minutes and achievement of steady-state was identified by monitoring the outlet total temperature. Once steady-state was achieved three measurement points were taken over a period of fifteen minutes, as specified by ASME PTC 10. A measurement point consists of all averaged quantities obtained from the specified 200 samples of each quantity. Each operating point indicated on the compressor performance characteristics shown in Chapter 7 was generated by taking the average of the three measurement points taken over the fifteen minutes of steady-state operation. During a given fifteen minute run it was common to see slight variations ( $<0.5 \%$ of the averaged measured quantity) in the compressor performance due to variations in the turbine blowing pressure and the resulting variation in the compressor rotational speed. Section 3.3 of PTC 10 gives limits to the maximum variations allowed in a given test measurement. Test points with variations larger than those specified by PTC 10 were rejected. The nozzle differential pressure measurements have an allowable $2 \%$ variation in the measured quantity as specified by PTC 10. However, at low flow rates, nozzle differential pressure measurements with up to $4 \%$ variation were accepted. These measurements were accepted because of the poor resolution on the flow velocity due to the square root relationship of Equation 4.1.

Roberts estimated the total measurement uncertainty of the existing instrumentation by summing the uncertainty of a single sample, single measurement (200 samples) and the typical variations during the fifteen minute test. The inlet and discharge total pressure measurements were estimated to be accurate to within $\pm 1 \%$ of the measured absolute pressure, the inlet and discharge temperatures are accurate to within $\pm 0.25 \%$ of the measured absolute temperature and the shaft speed is accurate to within $\pm 1 \%$ of the measured shaft speed. The uncertainty of the flow meter differential pressure increases significantly at lower flow rates. As a result, Roberts estimated the flow meter differential pressure to be accurate to within $\pm 1 \%$ of the measured differential pressure in 
most cases and accurate to within $\pm 3 \%$ of the measured differential pressure for tests at low flow rates $\left(\Pi_{m}<0.02\right)$.

Data acquisition of the new rotatable total pressure probes and static pressure taps, added as part of the test rig modifications, was performed using the same methodology and pressure transducer as the inlet and discharge pressure measurements. The new total pressure probes and static taps are located within the compressor casing upstream of the volute. It is therefore possible that measurements made by the new instrumentation are influenced by the impeller rotation. Depending on the shaft speed, approximately 690 to 840 blade passages will pass each rotatable total pressure probe and static pressure tap during the $0.1 \mathrm{~s}$ sampling time ( $200 \mathrm{samples).} \mathrm{This} \mathrm{corresponds} \mathrm{to} \mathrm{one}$ sample for every 3 or 4 blade passings. The measurements obtained are therefore a time average of a quantity which is varying with high frequency due to the turbulence in the flow and periodically at the blade passing frequency. Unfortunately it is difficult to estimate the uncertainty in this time averaging since it is affected by the pneumatic response of the instrumentation. Since the measurements from the new instrumentation were obtained in the same manner as the inlet and discharge total pressure, the measurement uncertainty of the new instrumentations is at best equal to that of the inlet and discharge pressure measurements (that is, $\pm 1 \%$ of the measured absolute pressure, as quoted earlier). However, the same level of accuracy is unlikely in the new instrumentation due to the higher frequency conditions and it is expected that the uncertainty is significantly higher than the values quoted for the inlet and discharge pressure.

There is additional uncertainty in the measurements of the rotatable total pressure probes due to the uncertainty of the spanwise position of the probe sensing hole and its angle relative to the radial direction of the rotor. The location of the probe sensing hole is estimated to be accurate to about $\pm 1 \mathrm{~mm}( \pm 0.04 \mathrm{in}$.). Each probe and collar pair was designed such that the probe is located spanwise by being butted up against the shroud 
wall of the compressor casing (the wall opposite the hole). This ensured that the probe sensing hole would always be in the same spanwise location, although the location is not precisely known because of possible unevenness of the wall surface. The probe sensing hole spanwise position is therefore estimated very approximately to be accurate to within $\pm 20 \%$ span.

The probe angle is set using indicating marks which have been etched into the larger cylindrical surface of the probe and probe collar. The probe collars are fixed to the compressor casing and the etched marks are aligned at a known angle relative to the radial axis. The uncertainty of the probe angle relative to the flow is therefore dependent not only on the manufacturing tolerances but also the probe collar installation and precision to which the probe sensing hole can be aligned relative to the probe collar. It was found that aligning the etched indicating mark of the probe to the indicating marks of the probe collar was significantly less accurate than either the manufacturing tolerances or the probe collar alignment relative to the radial axis. It is therefore believed that the uncertainty in the probe angle is primarily a function of the uncertainty in aligning the indicating marks on the probe to those on the probe collar. Each indicating mark spans approximately $2 \mathrm{~mm}$ of the $52 \mathrm{~mm}$ probe and probe collar circumference. The alignment of the indicating marks is therefore estimated to be accurate to within $\pm 1 \mathrm{~mm}$ which corresponds to approximately $\pm 7^{\circ}$ uncertainty in the specified probe angle.

\subsubsection{Uncertainties in Calculated Test Parameters}

The uncertainties in the calculated test parameters were estimated using the uncertainty analysis method of Moffat (1985). The author expresses the uncertainty in the calculated result $(R)$ as a function of the uncertainty in the measured test quantities $\left(x_{i}\right)$, by the following relationship: 


$$
\delta R=\sqrt{\left(\frac{\delta R}{\delta x_{i}} \delta x_{i}\right)^{2}}
$$

Results that are the products of measured quantities raised to some power can be expressed by:

$$
R=x_{1}^{a} x_{2}^{b} \ldots x_{n}^{m}
$$

The relative uncertainty $(\delta R / R)$ for calculated test parameters that take the form of Equation 4.20 can be found from:

$$
\frac{\delta R}{R}=\sqrt{\left(a \frac{\delta x_{1}}{x_{1}}\right)^{2}+\left(b \frac{\delta x_{2}}{x_{2}}\right)^{2}+\ldots+\left(m \frac{\delta x_{n}}{x_{n}}\right)^{2}}
$$

Table 4.3 summarizes the results of the uncertainty analysis when applied to the mass flow parameter, speed parameter, pressure ratio and efficiency. Estimates of the uncertainty in the mass flow parameter are given for both low and high flow rates since the relative uncertainty in the flow meter differential pressure increases at low flow rates. The error in the efficiency calculation is influenced by the pressure ratio and was therefore presented for representative values for each speed line. With the exception of the uncertainty in the efficiency calculation, all values have been taken from Roberts (2001). The estimated uncertainty in the efficiency is a function of the isentropic exponent and Roberts performed the uncertainty analysis for the efficiency calculation using argon, which had the highest isentropic exponent in his work. The uncertainty analysis for the efficiency has therefore been re-estimated using the isentropic exponent of air since all tests conducted within this study used air as the working fluid. 


\begin{tabular}{|c|c|}
\hline Test Parameter & Estimated Uncertainty \\
\hline Mass Flow Parameter & \\
$\Pi_{m}<0.02$ & $\pm 0.004( \pm 2.5 \% \mathrm{FS})$ \\
$\Pi_{m}>0.02$ & $\pm 0.0008( \pm 0.5 \% \mathrm{FS})$ \\
\hline Speed Parameter & $\pm 0.15( \pm 0.7 \% \mathrm{FS})$ \\
\hline Pressure Ratio & $\pm 0.03( \pm 1.3 \% \mathrm{FS})$ \\
\hline Efficiency: & \\
$\Pi_{N}=10.06$ & $\pm 3.3( \pm 4.4 \% \mathrm{FS})$ \\
$\Pi_{N}=15.23$ & $\pm 1.5( \pm 2.0 \% \mathrm{FS})$ \\
$\Pi_{N}=18.35$ & $\pm 1.1( \pm 1.5 \% \mathrm{FS})$ \\
\hline
\end{tabular}

Table 4.3: Estimated uncertainty in calculated test parameters

The method of Moffat (1985) is adequate for estimating the uncertainty in the calculated test parameters shown in Table 4.3. However, the same method cannot be applied to estimate the uncertainty in the impeller exit total pressure and flow angle.

The uncertainty in the impeller exit total pressure and flow angle is a function of the uncertainty in the individual measured values and the error in the curve fit used. As mentioned previously, the uncertainty in the measured pressures is greater than $\pm 1 \%$ and the uncertainty in the specified flow angle is about $\pm 7^{\circ}$. Typical R-squared values of the curve fit are in the range of 0.85 to 0.95 . This would suggest that the impeller outlet total pressure and flow angle estimates are accurate to within $\pm 1.5 \%$ and $\pm 10^{\circ}$ respectively. However, the uncertainty in the impeller exit total pressure and flow angle is likely much 
larger than the uncertainties in the individual total pressure and flow angle values due to the circumferential averaging process. It is therefore difficult to assign a value to the uncertainty in the impeller outlet total pressure and flow angle estimates. 


\subsection{COMPUTATIONAL METHODS}

\subsection{INTRODUCTION}

Aerodynamic performance predictions for two centrifugal compressors were obtained as part of the current study. The two compressors are the Turbonetics T04E-50 turbocharger compressor and the rotating test rig compressor designed in the present work. The performance predictions were obtained using the commercial CFD codes CFX-BladeGent and ANSYS CFX. The flows were predicted for several configurations of the test rig compressor including the impeller alone and the complete stage. This chapter details the computational methods used for the performance predictions.

The rotating test rig compressor was designed for use in an existing rotating test rig that is based on a turbocharger. The design process, detailed in Section 6.2, was performed using CFX-BladeGen. The capabilities of CFX-BladeGen and the computational methods used are discussed more thoroughly in Section 5.2. The test rig compressor was originally intended to validate the compressor aerodynamic performance predictions of the CFD software, ANSYS CFX. This would have been achieved by comparing the computational predictions to experimental data for the same compressor. Due to time constraints it was not possible to obtain the experimental performance data for the new test rig compressor. It was therefore decided to obtain the desired comparison using data from an existing machine. From the work of Roberts (2001) there existed a Turbonetics T04E-50 compressor ready for experimental testing. In addition, a three-dimensional scan of the rotor geometry provided an accurate solids-model representation for use with the computational software. It was therefore possible to use the T04E-50 impeller to obtain the measured data and use it to validate the compressor aerodynamic performance predictions of ANSYS CFX. The detailed aerodynamic performance of both the T04E-50 impeller and the rotating test rig compressor were 
predicted using ANSYS CFX. The computational methods used and a more detailed description of ANSYS CFX is given in Section 5.3.

\subsection{CFX-BLADEGEN}

CFX-BladeGen is commercially available software (since discontinued) with the distinct advantage of being both a geometry design (CAD) program as well as a fully three-dimensional fluids solver. The CAD module referred to as BladeGen is customized for the creation of turbomachinery models. Because of its ease of use, BladeGen is capable of generating small geometric changes to potential rotor geometries quickly. The CFD component known as BladeGen+ is customized for the rapid testing of potential turbomachinery designs and is based on the CFX-5 Navier-Stokes code. To ensure ease of use and rapid solving, several of the code's features are disabled or given default values. It is for this reason that the code is useful for preliminary design but insufficient for detailed aerodynamic performance predictions.

CFX-BladeGen was selected for the design of the rotating test rig compressor impeller discussed in Section 6.2. The goal was to determine an appropriate geometry that met specific manufacturing constraints while still maintaining as much of the original geometry and performance as possible. Since a previous preliminary rotor design was completed by Charest (2003), CFX-BladeGen was used for the rapid creation and testing of new geometries. Similarly when studying the effects of working fluid on the design geometry, rapid results of several geometric variants were required. Therefore CFXBladeGen was also selected for generating the results of Section 6.3.

The computational domain modelled in BladeGen+ consisted of a complete blade set with an extension of the flow passage at both the inlet and outlet. A complete blade set is comprised of a main and a splitter blade. Thus the geometry modelled is a one tenth circumferential section of the rotor since there is a total of ten blade sets in the 
complete rotor. The performance of the entire rotor is then based on the assumption of periodicity between blade sets. BladeGent does not have the capability to model tip clearances and thus none are present in the domain. The inability to model tip clearance and the resulting leakage flow is one of the major limitations of the software that prevent it from providing accurate aerodynamic predictions.

For simplicity BladeGen+ only allows the selection of either laminar or turbulent flow. Turbulent flow was selected and the code then makes use of a zero-equation turbulence model. The particular model used is not specified but has proved to be fast, robust and reasonably accurate for a wide range of problems (CFX-BladeGen+ User's Manual, 2000). Regardless, it is common practice to use turbulence models consisting of two equations or more to generate more detailed aerodynamic predictions of turbomachinery flows.

The mesh is composed of a structured prismatic mesh, known as inflation layers, next to the solid surfaces and an unstructured 'tet-mesh' made of tetrahedral elements elsewhere. When generated properly the inflation layers serve to capture the fluid effects in the boundary layer with fewer elements than a corresponding tet-mesh would require. Even with the use of two element types BladeGen+ is still limited in its ability to sufficiently mesh the computational domain because of the meshing options available. Again in an attempt to simplify case definition the meshing options only allow the selection of an overall grid refinement factor and number of inflations layers desired on the solid walls. The maximum number of cells or nodes is also limited in order to give rapid computations. Since BladeGen+ is tailored specifically to turbomachines the grid is significantly finer near the leading and trailing edges, however no other mesh control is accessible. As with any CFD computations it is important to obtain grid independent results. This would ensure that the design process demonstrates only the changes in performance due to changes in geometry. To achieve this, a grid dependence study was performed on the rotor detailed in Charest (2003). Since the geometry is very similar in 
the new design it is assumed that the grid refinement factor selected based on the design of Charest (2003) will be acceptable for both designs. To validate this assumption a grid dependence study was performed on the final design selected. The re-design process was conducted by performing parametric studies with specific geometric parameters and investigating their effect on the compressor performance characteristics. It was decided to use the effect of grid density on a constant speed operating line to determine grid sensitivity. Figures 5.1 and 5.2 show the total pressure ratio characteristics for the original impeller designed by Charest (2003) and the current test rig impeller for varying grid densities.

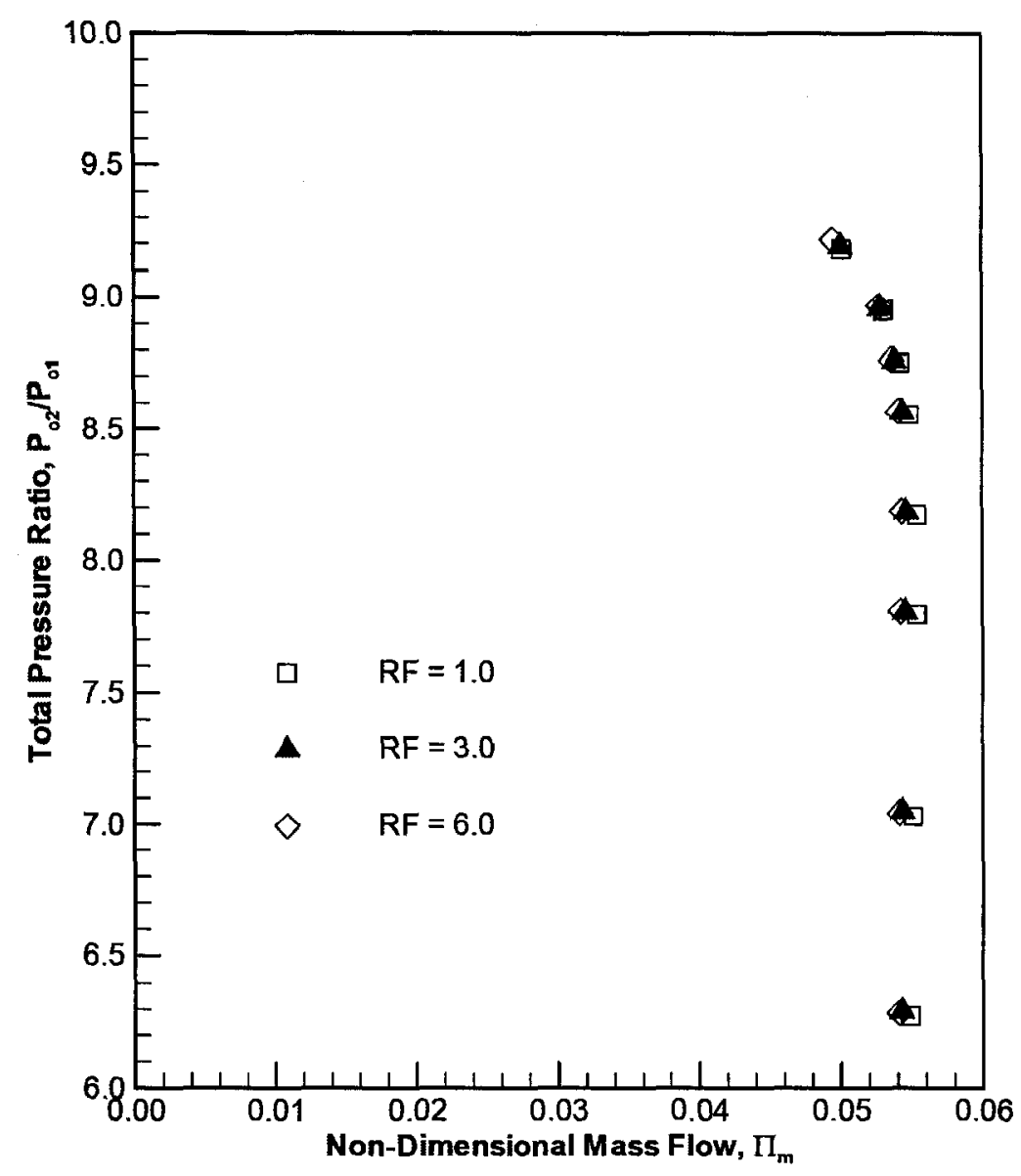

Figure 5.1: Charest (2003) impeller grid sensitivity in CFX-BladeGen+ 


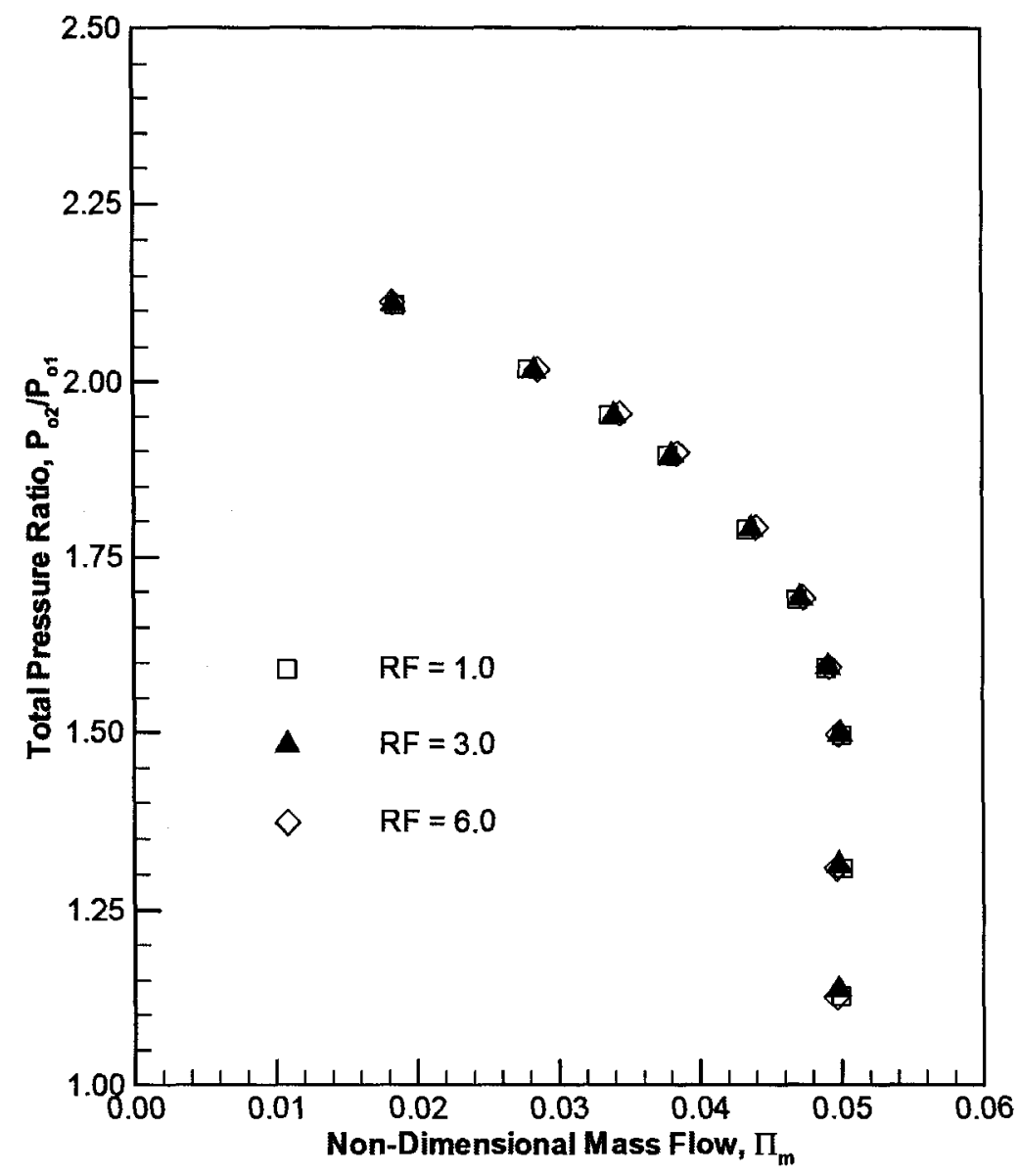

Figure 5.2: Rotating test rig impeller grid sensitivity in CFX-BladeGen+

It should be noted that the constant speed lines seen in Figure 5.1 and 5.2 do not correspond to the same rotational speed and therefore cannot be directly compared. Each operating point is specified by the rotational speed, inlet total temperature and total pressure and the outlet static pressure. As seen in both Figures 5.1 and 5.2 there is variation between the constant speed lines predicted with the three refinement factors and it is believed that BladeGen+ is not capable of generating a grid with sufficient density to achieve grid independent solutions. Nevertheless, BladeGen + should be capable of generating the trend accurate results required for the design process with either of the grid refinement factors shown. A grid refinement factor of 3.0 was selected to perform all 
subsequent BladeGen+ calculations because of the ability to obtain solutions for a complete constant speed line within 24 hours using a dual processor machine. Table 5.1 summarizes the grid properties and run time for various grid refinement factors. The run time has been estimated on a single processor for 750 iterations since typical solutions reached the desired convergence criteria by this point.

\begin{tabular}{|c|c|c|c|}
\hline $\begin{array}{c}\text { Refinement Factor } \\
\text { (RF) }\end{array}$ & $\begin{array}{c}\text { Node } \\
\text { Count }\end{array}$ & Elements & $\begin{array}{c}\text { Run Time } \\
\text { (750 Iterations) }\end{array}$ \\
\hline 1.0 & 23145 & 45868 & $2 \mathrm{hr} 25 \mathrm{~min}$ \\
\hline 3.0 & 47010 & 117757 & $4 \mathrm{hr} 40 \mathrm{~min}$ \\
\hline 6.0 & 75621 & 213292 & $7 \mathrm{hr} 15 \mathrm{~min}$ \\
\hline
\end{tabular}

Table 5.1: Grid properties and run time for various grid refinement factors in CFX-BladeGent

\subsection{ANSYS CFX}

\subsubsection{Description of the Software}

ANSYS CFX is a commercially available general purpose CFD code. It consists of five individual software modules that allow a complete CFD analysis from grid generation to post processing. The grid generation available in ANSYS CFX is more advanced than CFX-BladeGen+ and provides several user options to adequately mesh complex geometries. If desired the software is also capable of working with other standard mesh files and allows the use of multiple domains and mesh types in a single computational case. In addition, the physics definitions available in ANSYS CFX are also more detailed than BladeGent. ANSYS CFX provides a full range of boundary conditions, domain interfaces and turbulence models to analyze a wide variety of flow fields. The solver used is fully three-dimensional and is based on the Navier-Stokes differential equations which describe fluid flow. ANSYS CFX is therefore an 
appropriate tool for detailed aerodynamic performance predictions of the Turbonetics T04E-50 impeller and the test rig compressor.

\subsubsection{Modelling of the Turbonetics T04E-50 Impeller}

The computational domains of the T04E-50 impeller as modelled in ANSYS CFX are shown in Figure 5.3. To aid in visualization the surface meshes of the domains have also been shown. However, the details of the mesh will be discussed later. The complete computational case of the T04E-50 impeller consists of both a rotating impeller (blue and green) and a stationery diffuser (red). Although it is intended to investigate the performance of just the impeller, the diffuser domain is added to extend the boundaries of the simulation beyond the radial location of the total pressure probes installed on the compressor casing of the experimental apparatus. These rotatable total pressure probes, detailed in Section 4.4.1, function collectively to mimic the capabilities of a multi-hole probe and provide both total pressure and flow angle data. The addition of the diffuser domain therefore allows direct comparison of the total pressure probe data to the computational predictions.

Both domains are generated independently and are mated using a so-called Frozen Rotor interface. This type of interface changes the frame of reference while maintaining the relative position of the components and the inlet of the diffuser is therefore exposed to the rotor discharge flow at a fixed rotor position. The interface allows the wakes generated by the impeller blades to propagate into the diffuser but at a fixed circumferential position resulting in a steady-state solution in both reference frames. As a result the unsteady effects of the passing wakes are not modelled when using a Frozen Rotor interface. The Frozen Rotor interface was selected here because it is computationally the simplest of three options available in ANSYS CFX and the unsteady effects are believed to be small. 


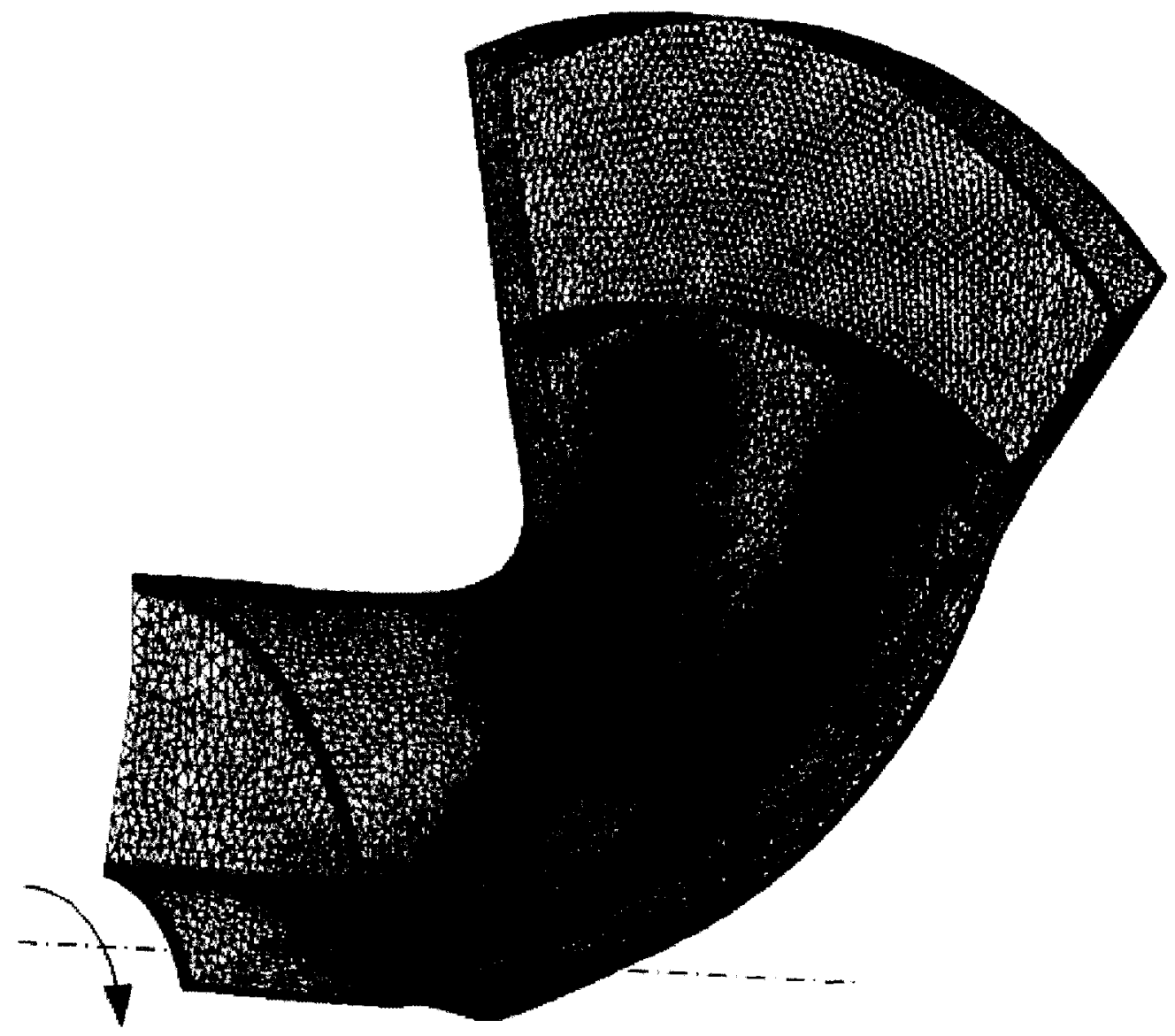

Figure 5.3: Computational domains for the Turbonetics T04E-50 compressor impeller

Conformal grids would give a one-to-one match between nodes across the impeller-diffuser interface and would produce the most accurate predictions. However, the use of conformal grids was not feasible based on the complex geometries used. Instead the General Grid Interface (GGI) connection provided by CFX was used. This connection allows differences in node locations as well as element types by enforcing conservation for all fluxes across the interface by appropriate interpolation techniques.

For the T04E-50 impeller, both domains modelled are a one sixth circumferential section of their respective components. This represents a complete blade set of the impeller and a $60^{\circ}$ annular section of the diffuser. A blade set is comprised of a main and 
splitter blade (illustrated with green in Figure 5.3) and the corresponding vane space (blue). The model also includes the clearance between the blade tips and shroud surface and was estimated as a constant $0.635 \mathrm{~mm}$. The impeller domain is also extended upstream of the inlet plane and downstream of the exit plane to ensure that the defined inlet boundary condition and domain interface are sufficiently far from the blade leading and trailing edges. This is recommended because these regions contain complex flow structures and their interaction with boundary conditions and domain interfaces may hamper convergence.

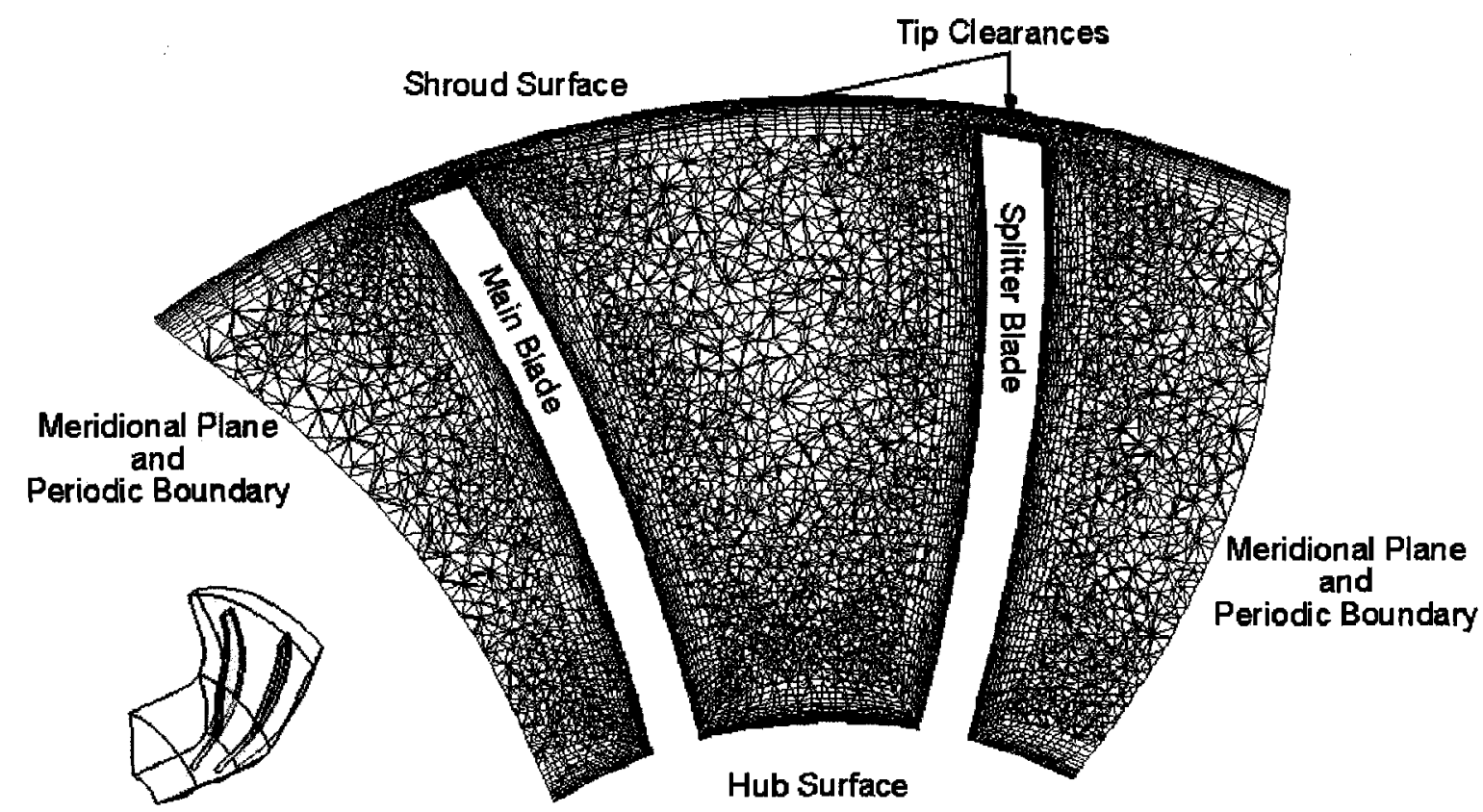

Figure 5.4: Slice plane of Turbonetics T04E-50 grid at constant axial location

Figure 5.4 shows the major features of the mesh on a slice plane at an arbitrary streamwise location. The mesh is composed of inflation layers next to the solid surfaces and an unstructured 'tet-mesh' elsewhere. Fifteen inflation layers are grown outward from a specified first node height off the wall, expansion ratio and maximum thickness on all surfaces such that they blend into the unstructured tet-mesh of the interior of the 
flow. The inflation layers generated resolve the boundary layer with the recommended minimum 10 nodes (ANSYS CFX User's Manual, 2005). This ensures the greatest accuracy for the k- $\omega$ based, Shear Stress Transport (SST) turbulence model used. The length scale of all elements reduces near the solid surfaces in order to accurately resolve the curvature of the complex geometry. There is also a greater concentration of nodes at both the blade leading and trailing edges. This is common practice for CFD predictions of turbomachinery because the aerodynamic performance is sensitive to the flow in these regions and care must be taken to properly resolve the geometry and flow.

An assumption of periodicity around the rotor is made and a periodic interface is defined at the two meridional planes. Unfortunately due to the overall length scale of the domain compared to the height of the first node off the wall it was not possible to incorporate a one-to-one node match across the periodic interface while still achieving appropriate boundary layer resolution. Instead, a GGI connection was also used at the periodic boundary.

Turbulence models based on the $k-\omega$ equations have a distinct advantage in predicting the onset and extent of boundary layer separation under adverse pressure gradients more accurately than other two equation models such as those based on the k- $\varepsilon$ equations. Unfortunately traditional $\mathrm{k}-\omega$ models lack the accuracy in free shear flows that the k- $\varepsilon$ models can provide (ANSYS CFX User's Manual, 2005). Therefore the SST model was chosen because it uses the $k-\omega$ model in wall regions and the $k-\varepsilon$ model away from the walls. This is achieved with blending functions in the near wall region that are functions of wall distance and local flow variables. The SST model requires that the values of $y+$ be less than about 2 at the first node away from the wall. $y+$ is based on the distance of the first node to the wall $(\Delta y)$ and the wall shear stress $\left(\tau_{w}\right)$ and is defined as:

$$
y+=\frac{\rho \Delta y U_{\tau}}{\mu} \quad \text { and } \quad U_{\tau}=\sqrt{\frac{\tau_{w}}{\rho}}
$$


$y+$ provides information on the boundary layer resolution and its value being less than 2 ensures that the viscous sublayer has been resolved. This method is known as a LowReynolds Number $(R e)$ method because of the extremely small mesh length scales normal to the wall (i.e. very thin inflation layers) needed. Although the SST model is suitable for a low-Re method it was not possible to generate a grid which met these requirements on all surfaces. However, ANSYS CFX uses an automatic near-wall treatment method for $\mathrm{k}-\omega$ based turbulence models. This treatment allows for a shift from the low-Re form to a wall function method which is claimed to be without loss of accuracy (ANSYS CFX User's Manual, 2005). The wall function method does not rely on complete resolution of the boundary layer because it uses an empirical formulation known as the "law of the wall' to determine appropriate flow conditions near the wall.

To fully define the flow problem the inlet was specified using a total pressure and total temperature boundary condition while the exit plane boundary condition was specified as an opening using an averaged static pressure. An opening differs from an outlet in that it allows fluid to both exit and enter the domain. This is required because the presence of reversed flowing fluid at lower mass flow operating points would prevent the solver from converging to a solution if an outlet boundary was specified. Multiple operating points were investigated by varying the exit static pressure for a given inlet condition and thus altering the mass flow through the impeller. A solution was deemed converged when the RMS error residuals of the momentum and mass equations were less than $10^{-6}$. This resulted in the maximum residuals being less than $10^{-5}$ for the majority of cases studied. In two cases the RMS residuals were approximately $10^{-4}$. This occurred at operating points near choking and the higher residuals were limited to a very small region. It is therefore believed to be a localized phenomenon that is preventing complete convergence and the characteristics of the overall performance parameters, seen in Section 7.4.1, appear to be unaffected. All solutions were obtained using a blended second order advection scheme known as High Resolution available in CFX. The High Resolution advection scheme attempts to use second order accuracy everywhere. In 
regions where second order accuracy may lead to convergence problems (i.e. regions with high gradients) this scheme automatically switches to a blend between first and second order accuracy. The accuracy is reduced towards first order only as much as needed to allow convergence.

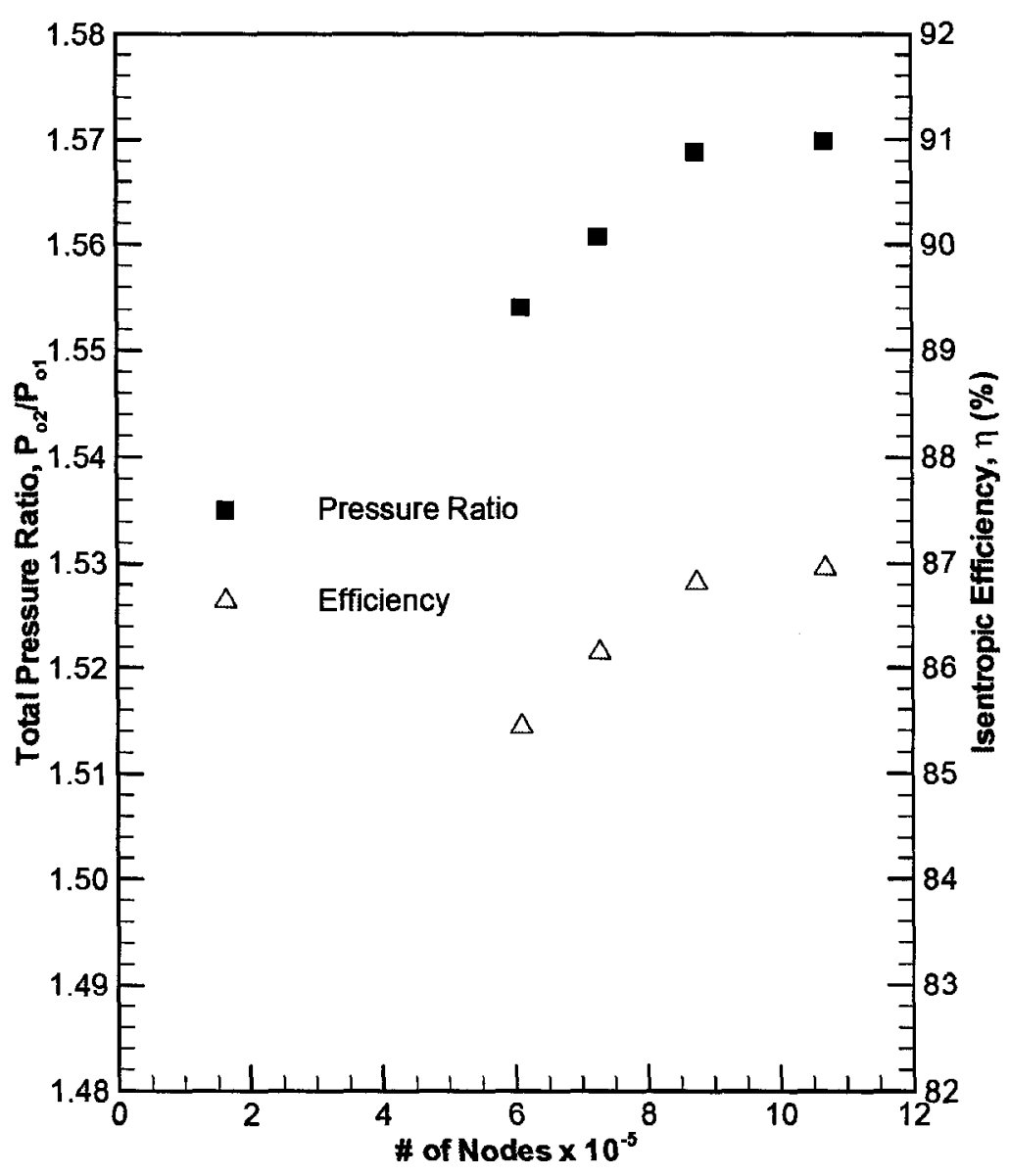

Figure 5.5: Turbonetics T04E50 grid sensitivity in ANSYS CFX

To ensure solutions were obtained that were grid independent a grid sensitivity study was conducted. Figure 5.5 shows the variation of pressure ratio and efficiency with node count. It was found that the overall stage performance parameters became insensitive to grid density with approximately 900,000 nodes. This applies to the 
pressure ratio and efficiency as seen in Figure 5.5 in addition to the mass flow rate which has not been plotted. These parameters were selected because they are the key aerodynamic parameters that characterize the overall performance of the impeller.

\subsubsection{Modelling of the Test Rig Compressor Impeller}

The computational methods used for the test rig compressor impeller were nearly identical to those for the Turbonetics T04E-50 impeller detailed in Section 5.3.2. This is beneficial because it is the CFD analysis of the T04E-50 impeller that will be validated by experimental data. In order to utilize the relationship between experimental data and computational predictions obtained from the T04E-50 impeller it is useful to maintain as much similarity between the computational methods for the two impellers.

The domain used consists of a complete blade set which extends outward from both the leading and trailing edges of the blade passage. Unlike the computational case of the T04E-50 impeller a separate diffuser domain was not modelled and therefore similar downstream lengths have not been modelled. As mentioned the diffuser domain was used in the T04E-50 computations to allow direct comparison to the measured data. Because experimental data were not collected for the test rig impeller a similar downstream length was not needed. Again a GGI interface was used at the periodic boundaries. The SST turbulence model and the High Resolution advection scheme were used as before. A constant $1 \mathrm{~mm}$ tip clearance between the blades and shroud surface was modelled to represent the clearance between the impeller and its casing. A separate computational case for the test rig impeller was generated with zero clearance between the blade tips and shroud surface in an attempt to understand the effect of tip leakage on the performance of the impeller.

A grid sensitivity study was conducted similar to that for the T04E-50 case using the variation of total pressure, efficiency and mass flow rate to judge the grid dependence. It was found that both test rig impeller cases (with and without tip 
clearance) became grid independent with approximately 450,000 nodes. This is approximately half the nodes required for the T04E-50 domain. It is reasonable that the test rig impeller requires about half the number of nodes considering the larger exit blade height to blade passage length and additional diffuser used for the T04E-50 impeller computations. For both test rig impeller cases over half of the solutions would reach convergence with RMS residuals less than $10^{-6}$. However, the solutions for operating points at or near the choking mass flow rate would only reach residual values between $10^{-3}$ and $10^{-5}$. This was a result of high residuals in small regions of the domain. Nevertheless, an attempt to lower the residuals was performed by solving the affected operating points using time accurate calculations available in ANSYS CFX. It was found that complete convergence could be achieved. It is therefore believed that the regions of high residuals are associated with flow structures that change with time. On the other hand, it was found that the steady-state solutions matched all major performance parameters of the time accurate solutions with less than $0.5 \%$ difference. This is likely because the regions of high residuals represented a small portion of the domain. It was therefore decided that steady-state solutions would be adequate since the accuracy was satisfactory and the convergence time was significantly shorter.

\subsubsection{Modelling of the Test Rig Compressor Stage}

The CFD analysis of the rotating test rig compressor was also applied to the full stage. In preparation for future experimental testing of the impeller, a volute and casing had also been designed and manufactured. It was therefore possible to model and solve the entire stage in ANSYS CFX. This was done using the existing domain from the impeller case with tip clearance modelled. The domain was then repeated about the rotational axis to create the complete impeller. The outlet of the impeller was mated to the inlet of the diffuser/volute section and an outlet was defined at the volute exit plane. Figure 5.6 shows the complete stage modelled in ANSYS CFX. The regions shown in green represent high residuals and the significance of this will be discussed later. 


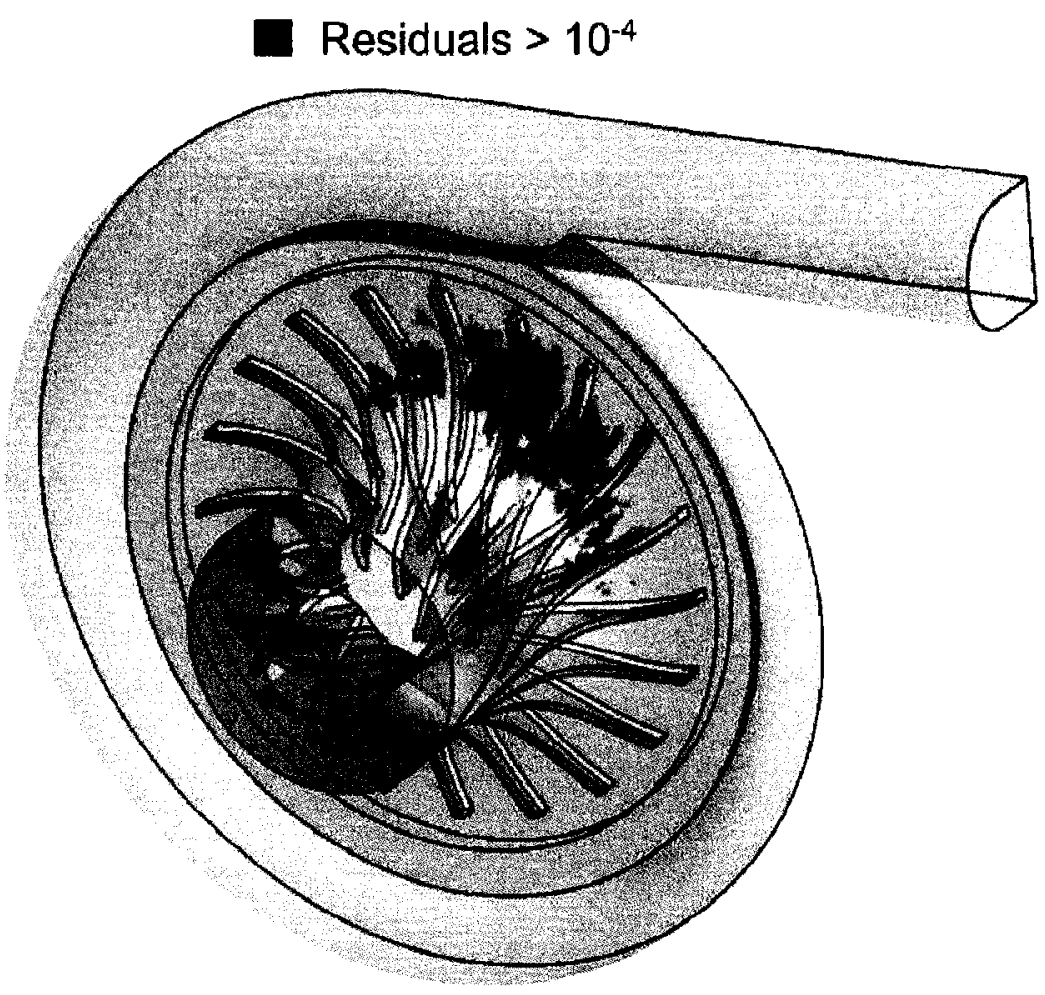

Figure 5.6: Computational domains of the test rig compressor stage in ANSYS CFX

Again the SST turbulence model and High Resolution advection scheme were used. It was found that the diffuser and volute section forced flow structures that were not periodic around the impeller. This lack of circumferential periodicity could only be detected by modelling the full impeller and the diffuser and volute. It demonstrates that some caution must be used when taking advantage of geometric periodicity, although this is often done to take advantage of the reduced node count and resulting shorter run time. These results and other aerodynamic predictions are discussed further in Section 8.3. With the addition of the volute domain the node count for the entire stage was approximately 5 million nodes. Due to hardware limitations it was impractical to solve the case using time accurate calculations. Thus, all solutions presented later were steadystate and therefore also used the Frozen Rotor reference change at the interface between the impeller and volute. 
Since unsteady effects were present, the solutions could only be obtained with RMS residuals of approximately $10^{-4}$ when assuming steady-state conditions. This averaged value is the result of localized regions of high residuals and does not accurately represent the majority of the domain. Figure 5.6 shows the elements with residuals that were greater than $10^{-4}$. This represents a typical solution and as seen only a small number of elements have error residuals greater than $10^{-4}$. It is therefore assumed that these unresolved unsteady effects do not prevent the solution from providing reasonably accurate overall stage performance data which is the most important goal of the computational analysis. 


\subsection{INFLUENCE OF WORKING FLUID ON COMPRESSOR DESIGN GEOMETRY}

\subsection{INTRODUCTION}

The preliminary design of the Raven Zero-Emission Gas Turbine (RZEGT) compressor impeller was performed by Charest (2003). The impeller was designed to meet the target performance parameters set out by the Raven Zero-Emission Gas Turbine performance specifications. Due to manufacturing difficulties the preliminary geometry had to be modified by the present author before manufacturing could occur. In addition, the modified geometry was designed such that it could be installed in an existing rotating test rig. To facilitate the design process it was desired to produce a geometry that allowed manufacturing within the given constraints while still maintaining as much of the original performance as possible. This would provide an impeller, ready for experimental testing, to validate corresponding CFD predictions for the same geometry.

The current chapter begins by detailing the design process used to modify the existing preliminary design to obtain the current impeller geometry. With the design for manufacturing complete the results of the preliminary CFD performance predictions of the current impeller geometry are presented and discussed. As described in Chapter 5.0 the original intent was to provide both experimental and computational results for the current geometry but time constraints prevented the experimental performance data from being collected. Nevertheless, it is appropriate to name the current impeller geometry as the rotating test rig impeller, in reference to its intended use. Since the experimental results could not be provided for the current geometry, experimental data were instead obtained for an existing Turbonetics T04E-50 centrifugal compressor impeller. In addition, a number of improvements were made to the test rig in preparation for future testing of the test rig impeller. These test rig modifications are discussed in Section 4.4 
and the subsequent experimental measurements are presented in Section 7.3. The measured data are also compared with detailed CFD predictions in Section 7.4. Since the preliminary design was intended for use with $\mathrm{CO}_{2}$ it also provided an opportunity to investigate the effect of working fluid on the compressor design geometry. This was done by designing an alternative geometry which preserved all non-dimensional parameters as the $\mathrm{CO}_{2}$ compressor but was designed to compress air. The new geometry shows that the change in performance due to the change in working fluid can be counteracted with simple geometric modifications. These results are given in Section 6.3.

All of the CFD predictions documented in this chapter were obtained using CFXBladeGen+. In addition, the re-design process of Section 6.2.1 and the geometric modifications presented in Section 6.3 also involved the use of the CAD component of CFX-BladeGen. This software was selected for its ability to rapidly modify turbomachinery geometries as well as quickly and easily perform approximate CFD predictions. The CFD component of CFX-BladeGen is easy to use but is not capable of producing highly accurate CFD predictions but can give quick preliminary results that are expected to be trend accurate. It is therefore useful for the comparison of multiple geometries in the re-design process as well as investigating the effect of an altemative working fluid on the design geometry. A more detailed description of the computational software and methodology is given in Section 5.2.

\subsection{Design Of the ROtATING TEST Rig IMPELLER}

\subsubsection{Impeller Design for Manufacture}

As described by Hunt (2004), the impeller designed by Charest (2003) needed to have its blades thickened while further increasing the overall vane spacing by about $25 \%$ to allow the rotor to be manufactured. The only means of meeting this requirement was to reduce the overall number of vanes. Since the redesign began from an existing geometry it was decided to conduct several parametric studies on the effect on the 
aerodynamic performance of changing specific geometric parameters. These studies were used to investigate the influence of blade count, blade thickness and wrap angle on the overall performance characteristics of the impeller. Since the reduction in blade count would likely lower the performance of the impeller it was hoped that these investigations would yield an acceptable geometry that maintained as much of the original performance as possible. The main performance parameters for the preliminary design by Charest (2003) are summarized in Table 6.1 along with the original design targets specified by the Raven Zero-Emission Gas Turbine project.

\begin{tabular}{|c|l|c|c|}
\hline Symbol & Parameter & $\begin{array}{c}\text { Design } \\
\text { Requirement }\end{array}$ & $\begin{array}{c}\text { Preliminary } \\
\text { Design (Charest) }\end{array}$ \\
\hline$\dot{m}$ & Mass Flow Rate $\mathrm{CO}_{2}$ & $0.444 \mathrm{~kg} / \mathrm{s}$ & $0.447 \mathrm{~kg} / \mathrm{s}$ \\
\hline$P R$ & Total Pressure Ratio & 8.0 & 9.03 \\
\hline$\eta$ & Isentropic Efficiency & $86.3 \%$ & $87.5 \%$ \\
\hline$T$ & Power Input & $73.7 \mathrm{~kW}$ & $81.1 \mathrm{~kW}$ \\
\hline$N$ & Rotational Speed & $110,000 \mathrm{RPM}$ & $110,000 \mathrm{RPM}$ \\
\hline
\end{tabular}

Table 6.1: Summary of performance parameters for the preliminary impeller designed by Charest (2003)

To begin the re-design process it was first necessary to examine the effect of the number of blades on the impeller aerodynamic performance. Figures 6.1 to 6.3 depict the effect of blade count on the total pressure rise, slip factor and efficiency of the rotor as predicted by CFX-BladeGen + . All calculations were performed at a constant rotational speed of 110,000 RPM. BladeGen+ outputs the total pressure ratio, efficiency and mass flow rate directly in a summary table of performance parameters. The slip factor, defined below, is calculated separately from mass-averaged velocity values at the trailing edge.

As can be seen, the predominant effect of a reduced blade count is to increase the choking mass flow rate with a reduction in blades. This is the result of the reduced flow 
blockage and thus larger throat area present at the impeller inlet with fewer blades of a given thickness. The design mass flow rate is also apparently closer to surge (i.e. the surge margin is reduced) as the blade count is reduced while preserving all other geometric parameters. One should note that surge is a system instability and therefore impossible to predict accurately with only the impeller characteristics shown. It is known from experience that BladeGen+ can only provide converged solutions above some minimum mass flow rate. It is believed that lack of convergence may indicate the onset of blade stall. Surge would only follow if all blades stalled and a sufficient backpressure was present to completely reverse the flow direction through the compressor. These conditions, and therefore surge, are impossible to predict with the current computational case. But since the onset of stall is a prerequisite for surge, any trends in blade stall seen in the characteristics can be interpreted as trends in surge onset.

Although a particular surge margin was not specified as a design requirement it is nonetheless an important factor to consider for impeller designs. The off-design performance is directly affected by the surge margin and is therefore important to many compressor applications. By decreasing the inlet vane height it would be possible to reduce the choking mass flow rate and therefore recover the original operating range. However this modification was not investigated because subsequent geometric changes also have an effect on the operating range of the impeller. In addition, the test rig impeller will only be used for future experimental testing and the off-design performance is not of great concern. In fact, the occurrence of surge during the experimental testing could provide useful data. 


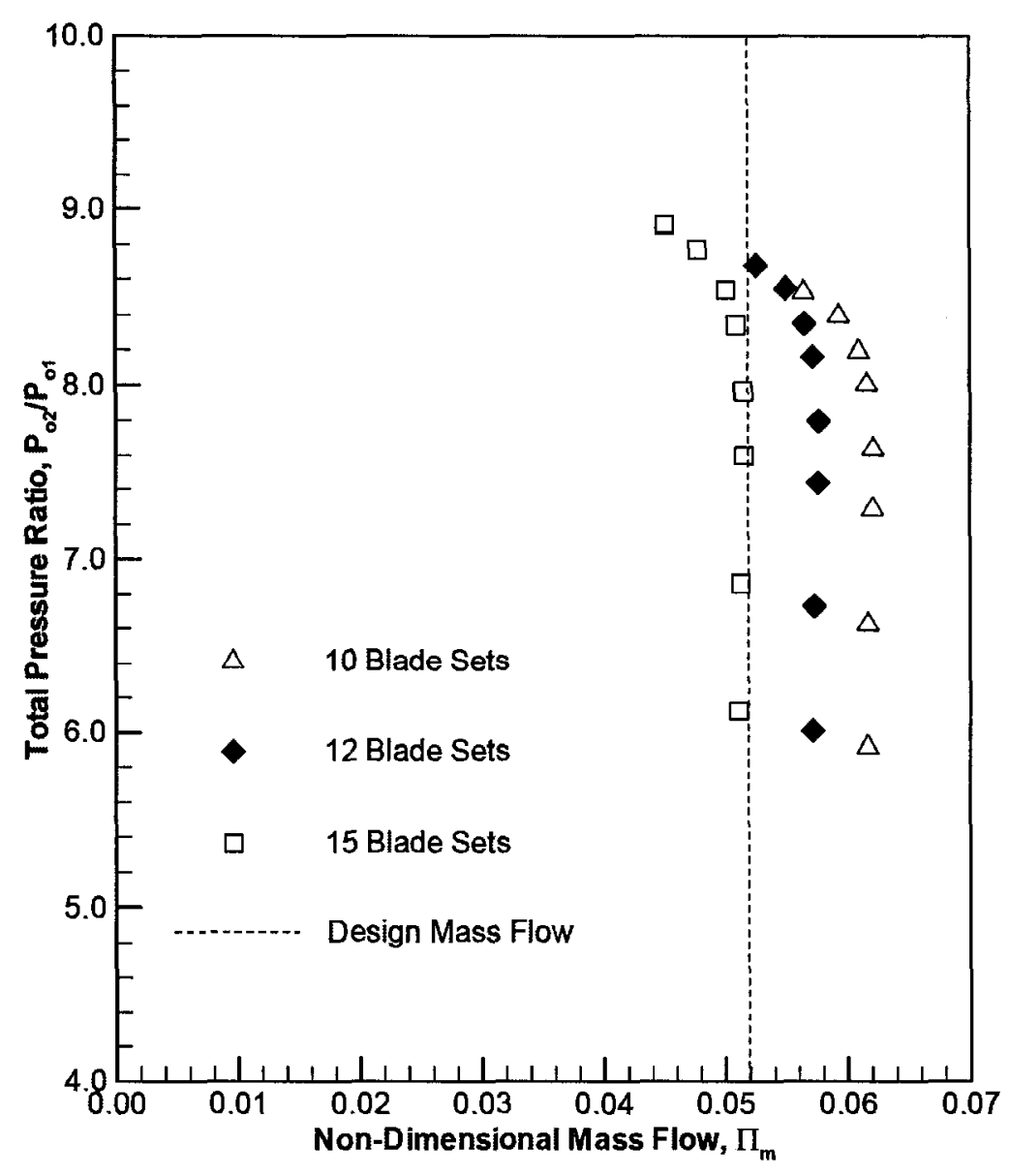

Figure 6.1: Influence of blade count on impeller pressure ratio

As seen from Figure 6.1, the maximum total pressure rise is decreased with the reduction in the number of blade sets, where a blade set consists of a full blade and a splitter blade. It is possible that the reduced pressure rise is partly a consequence of the increased blade loading, which has the potential to increase trailing edge separation. However, one would also expect a reduced pressure rise due to the observed decrease in the slip factor, $\sigma$ (Figure 6.2). The slip factor is defined as the ratio of the actual to the ideal tangential velocity.

$$
\sigma=\frac{C_{2 \theta}}{C_{2 \theta}^{\prime}}=\frac{C_{2 \theta}}{U_{2}-C_{2 r} \tan \left(\beta_{2}\right)}
$$


With the denominator being constant for a given rotational speed $U_{2}$, mass flow rate and outlet blade angle $\beta_{2}$, it is apparent that the decreased slip factor corresponds to a decreased outlet tangential velocity. From the Euler equation this results in less ideal work being delivered to the fluid and therefore a smaller pressure ratio:

$$
\Delta h_{o}=U_{2} C_{\theta 2}-U_{1} C_{\theta 1}=U_{2} C_{\theta 2}
$$

There is no inlet swirl $\left(C_{\theta 1}=0\right)$.

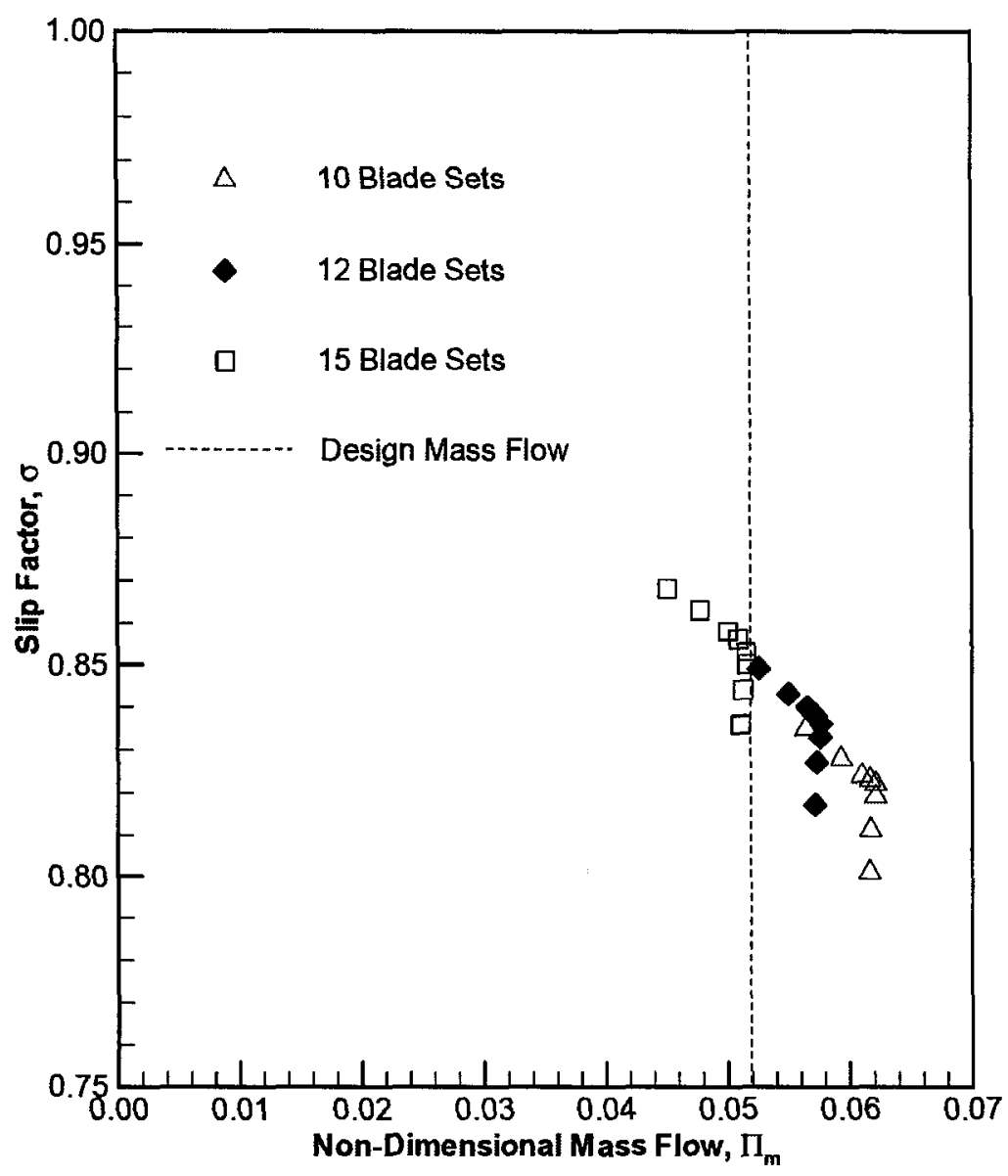

Figure 6.2: Influence of blade count on impeller slip factor 
It is known that the slip factor decreases with increasing blade loading without necessarily being associated with increased separation. It is therefore possible that the reduced pressure ratio with reduced blade count is the result of both reduced slip factor and increased flow separation. Since total pressure losses vary with the velocity squared it is to be expected that an impeller with a lower slip factor has slightly lower losses while separation tends to increase losses.

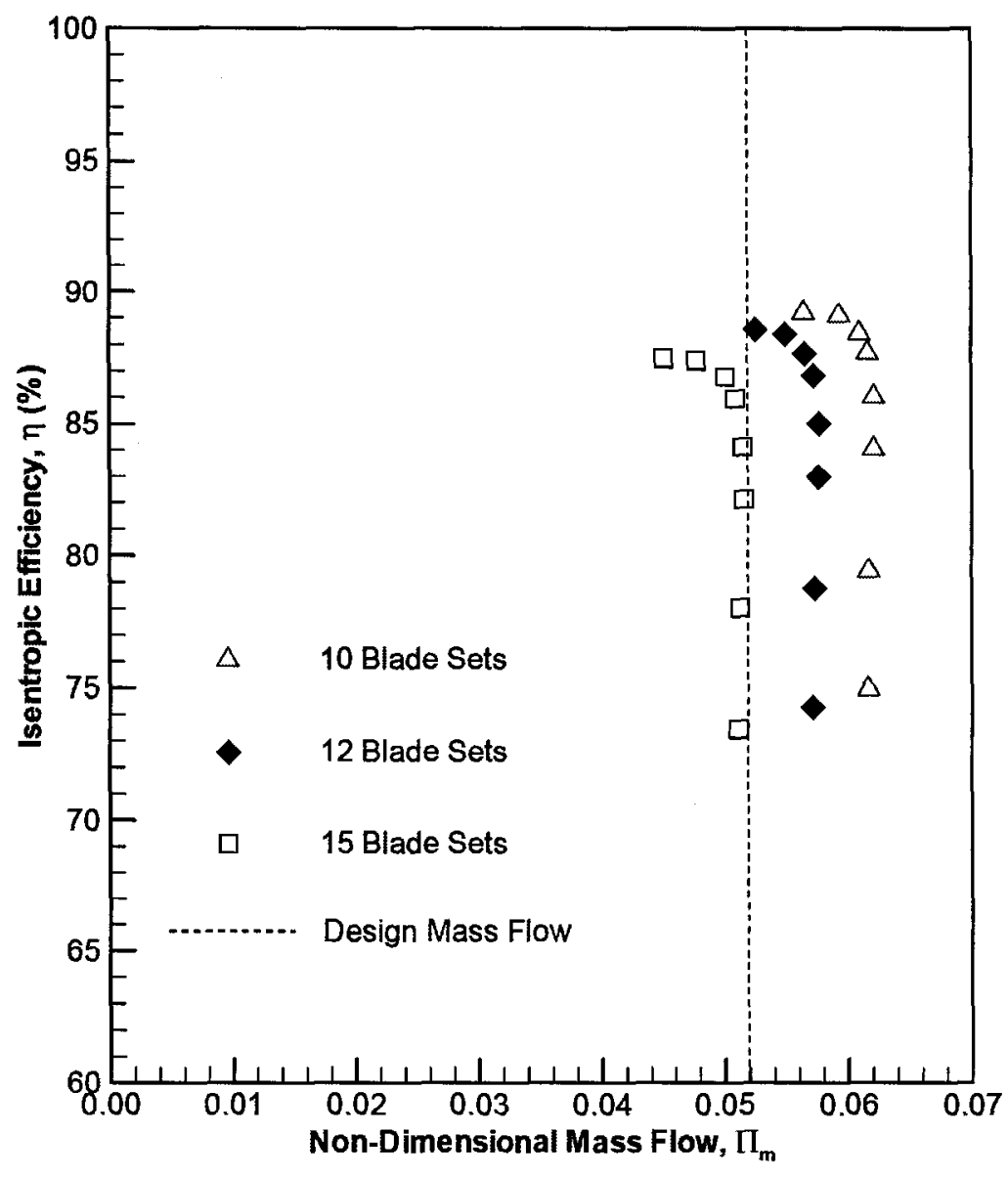

Figure 6.3: Influence of blade count on impeller efficiency

Figure 6.3 shows that the predicted efficiency increased with the reduced blade count, which suggests that the reduced pressure rise is primarily due to the reduced flow turning in this case. However, the reduced blade count has an effect on other geometric 
properties which makes it difficult to be certain of the predominant effect. For example, the reduced blade count also results in a decreased surface area which tends to lower the skin friction pressure losses. With the current software it is not possible to separate the effects and it is therefore difficult to determine the precise reason for the decreased total pressure rise. It is however evident that to achieve a maximum pressure rise the slip factor should be maintained at as high a value as feasible.

Due to convergence problems with CFX-BladeGent it is difficult to predict operating points at lower mass flow rates than those illustrated. As the operating mass flow rate is reduced fluid attempts to re-enter the domain at the outlet plane. Since only an outlet boundary (as opposed to inflow/outflow boundaries, which are allowed in the full ANSYS CFX) can be specified in BladeGen+, the solver attempts to correct for this by generating artificial walls along the outlet plane. Such cases would reach similar convergence criterion as other operating points however the validity of such a solution is questionable. It is therefore expected that the constant speed lines of all the rotor geometries may extend to lower values of mass flow rate. Based on the results given here together with subsequent investigations it was decided to use 10 blade sets since this yields a sufficient increase in vane spacing and is close to meeting the desired performance at the design mass flow rate.

With the vane spacing set to an acceptable value, the influence of blade thickness was then examined. Another requirement from the manufacturer was to at least double the thickness of the originally $0.5 \mathrm{~mm}$ thick blades to add strength to the pre-cast moulds. By doing so the flow blockage is increased and the choking mass flow rate is reduced as indicated by Figures 6.4 and 6.5 which show the total pressure ratio and efficiency characteristics with varying blade thickness. Increasing blade thickness simultaneously decreases the mass flow rate at stall, and perhaps at surge, and shifts the operating range. The maximum total pressure ratio is reduced as the blade thickness is increased. This is most likely a result of increased pressure losses due to a larger trailing edge thickness. 
This effect is also evident in Figure 6.5, which shows a loss in efficiency as the overall blade thickness is increased. As expected the larger sudden expansion in area at the trailing edge has increased the losses. It was decided to use a blade thickness of $1.25 \mathrm{~mm}$ as it did not significantly decrease the total pressure rise or efficiency while providing an acceptable blade thickness for manufacturing purposes.

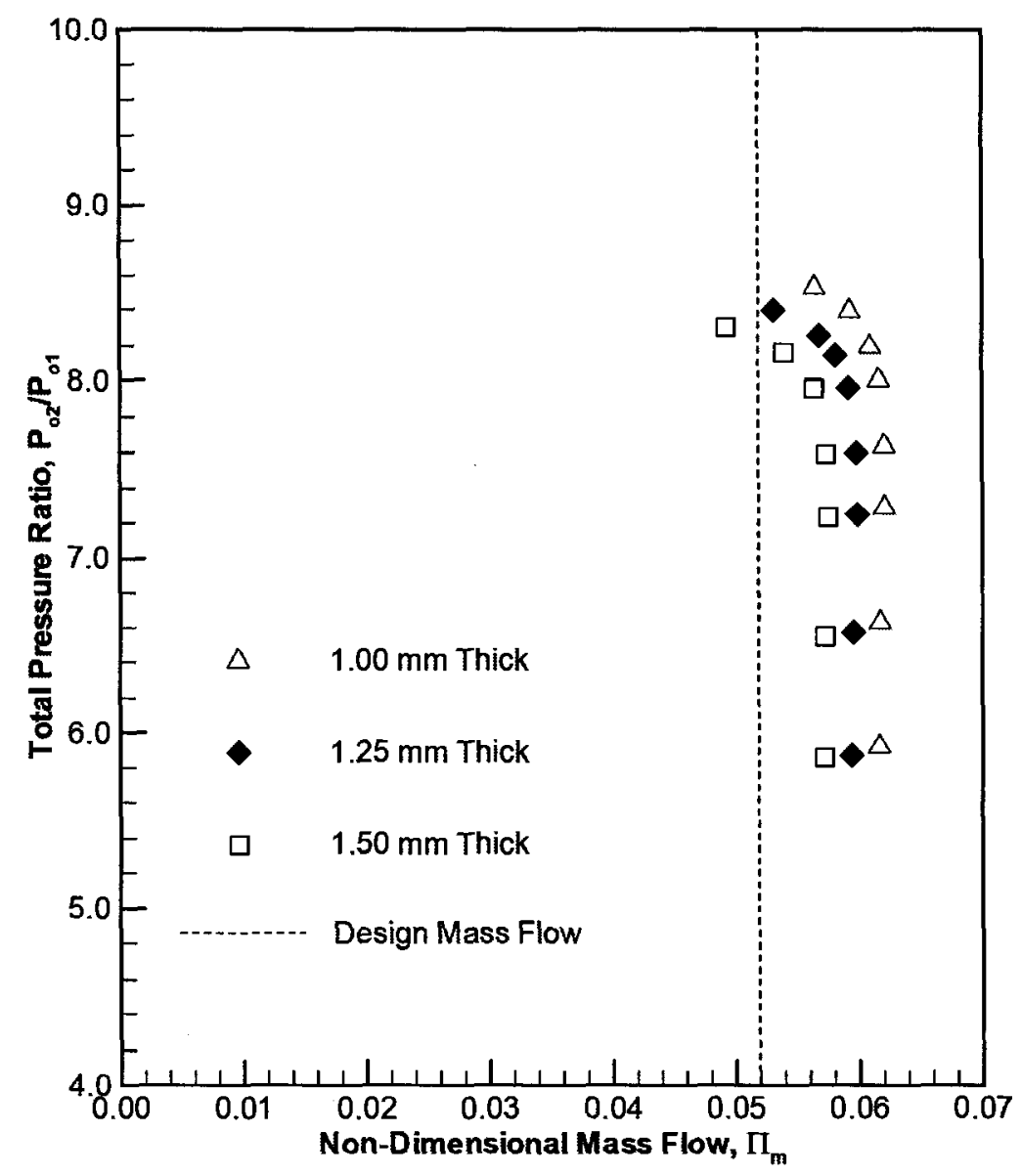

Figure 6.4: Influence of blade thickness on impeller pressure ratio 


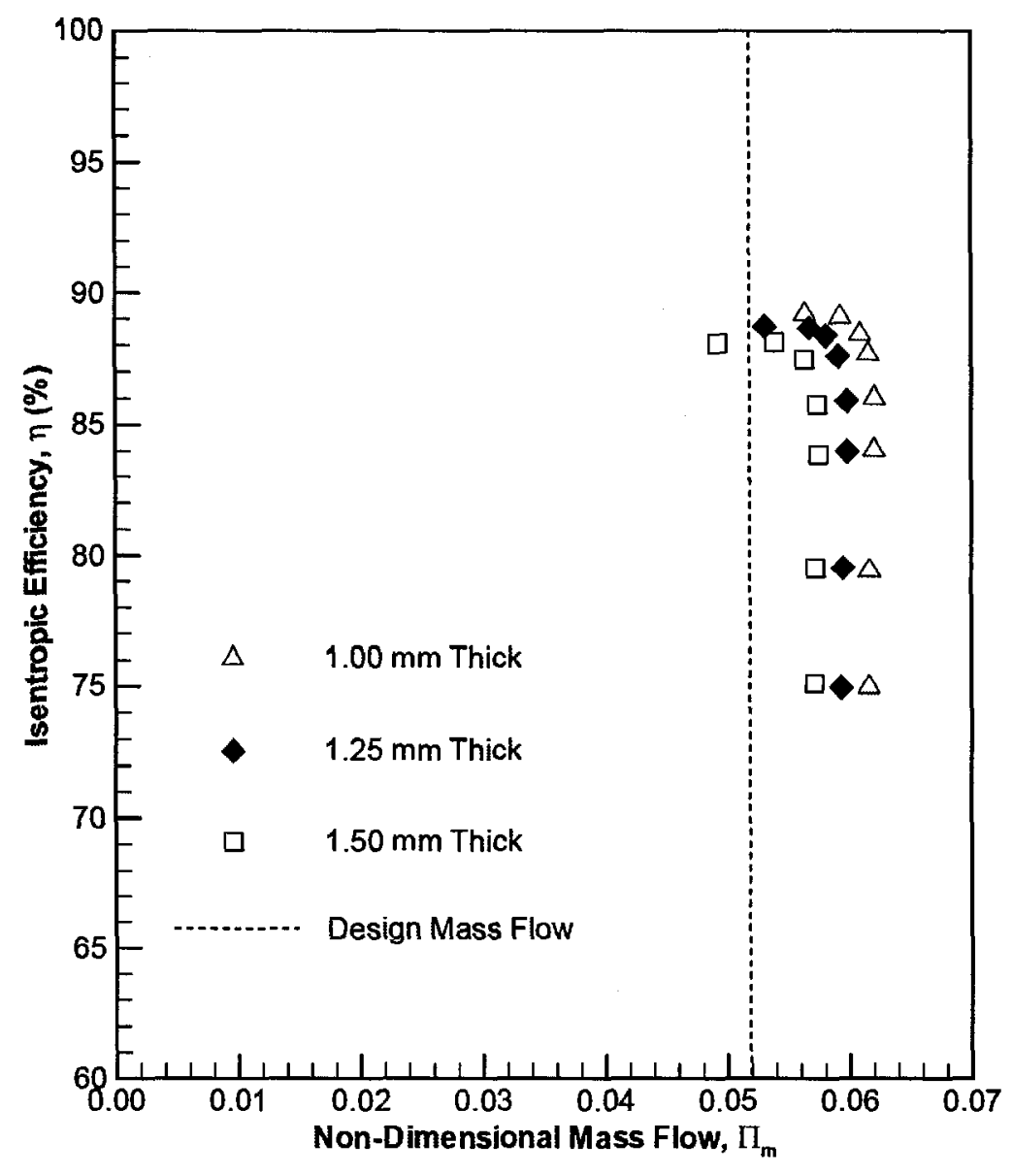

Figure 6.5: Influence of blade thickness on impeller efficiency

Increasing the vane spacing has increased the blade aerodynamic loading. Since the current geometry is still producing a pressure ratio higher than required and in an attempt to maintain the high efficiency it was decided to reduce the blade loading. This can be achieved by increasing the wrap angle $(\lambda)$ for the vanes. The wrap angle, shown schematically on the inset in Figure 6.6, is defined as the circumferential angle between the blade leading and trailing edges. Thus a larger wrap angle implies a longer blade passage and it is therefore possible to lower the blade loading without increasing the number of vanes. It was also hoped that the reduction in blade loading would increase the operating range of the compressor. 


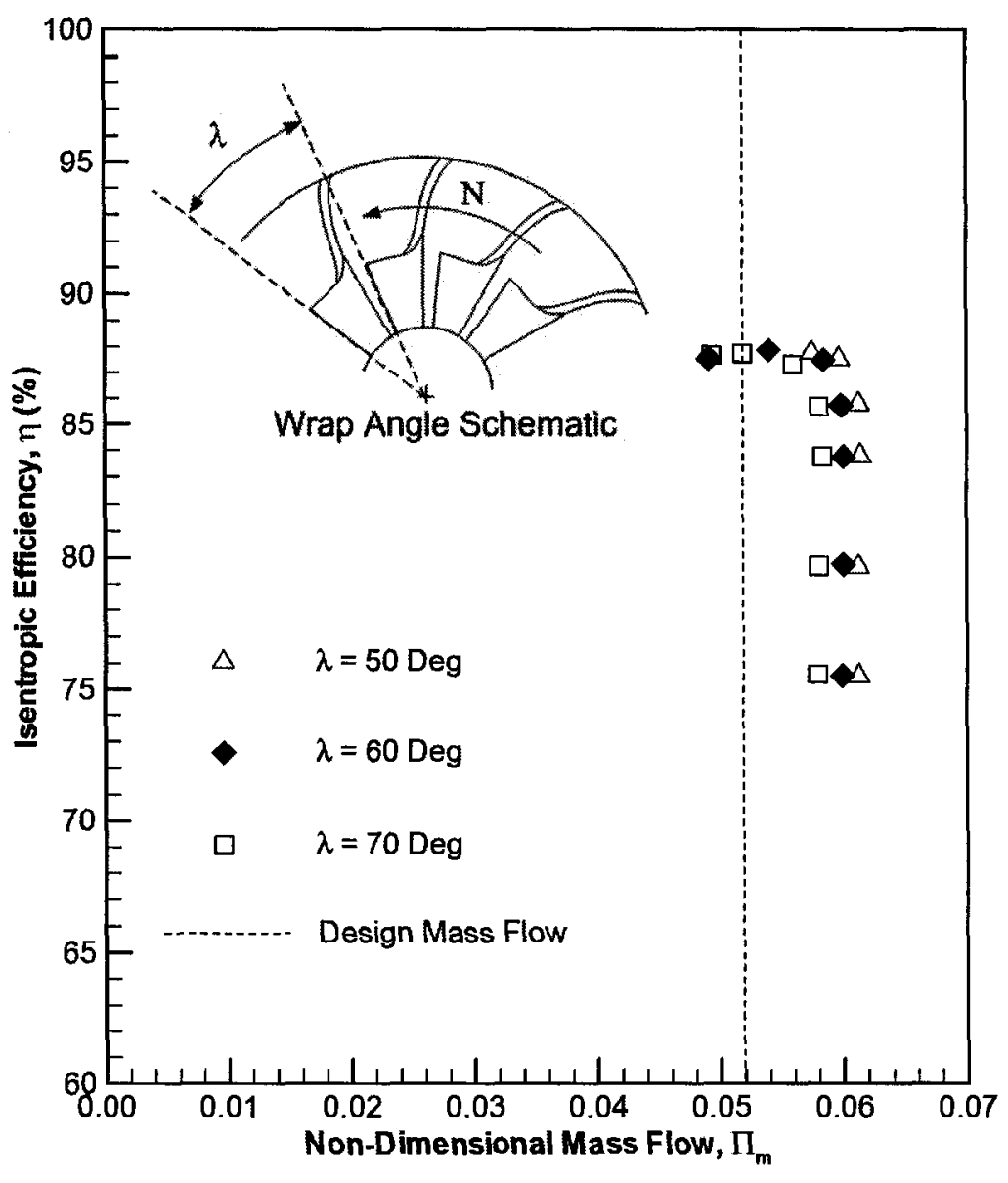

Figure 6.6: Influence of wrap angle on impeller efficiency

From Figure 6.6, which shows the effect of wrap angle on the efficiency characteristic, it is apparent that varying the wrap angle from $50^{\circ}$ to $70^{\circ}$ would give an operating line that includes the desired mass flow rate but does not change the predicted maximum efficiency. Unfortunately any reduction in losses resulting from the reduced blade loading has apparently been offset by an increase in skin friction losses on the larger wetted area of the vanes due to the larger wrap angle. Since it had little effect on the pressure rise the selection of a $70^{\circ}$ wrap angle was made only because it should provide a stable operating point, at the required mass flow, without increasing the wrap angle further. 
Initially the re-design was conducted for an impeller for use in the Raven ZeroEmission Gas Turbine (RZEGT). It was at this stage that it was realized that the original geometry was inappropriate for the existing turbomachinery test rig since the RZEGT impeller rotates in the opposite direction to that available in the test rig. Fortunately a mirrored geometry could be created with relative ease in CFX-BladeGen and mirroring has no impact on the performance predictions.

All the modifications made combined to increase the overall vane spacing for the test rig impeller by more than the required $25 \%$ needed for manufacturing. The major geometric parameters are summarized in Figure 6.7 and Table 6.2. The table also includes the corresponding values for the original design by Charest (2003). The changes made result in an approximate $10 \%$ reduction in total pressure rise and about a $2.0 \%$ reduction in predicted isentropic efficiency at the design flow rate. Thus the final design geometry does not meet all of the performance requirements of the RZEGT project as shown in Table 6.3. Although the current geometry is predicted to achieve the required total pressure rise it is expected that this too will fall short of the design requirements. For reasons discussed previously, the BladeGen+ results are only expected to be trend accurate and were used to estimate the change in performance during the re-design process and not expected to give accurate absolute predictions of the performance. In particular, it is expected that CFX-BladeGen+ will overestimate both the efficiency and pressure ratio due to its neglect of tip clearance leakage and the corresponding losses. Again a more detailed discussion of the computational software is given in Section 5.2 and more accurate CFD predictions of the current design using the full ANSYS CFX are presented in Chapter 8. As stated earlier, the purpose of the re-design process was to produce a manufacturable impeller suitable for experimental testing to be used to validate CFD predictions. Since the current impeller geometry does not meet the requirements of the RZEGT compressor impeller it is more appropriately known as the rotating test rig impeller. 


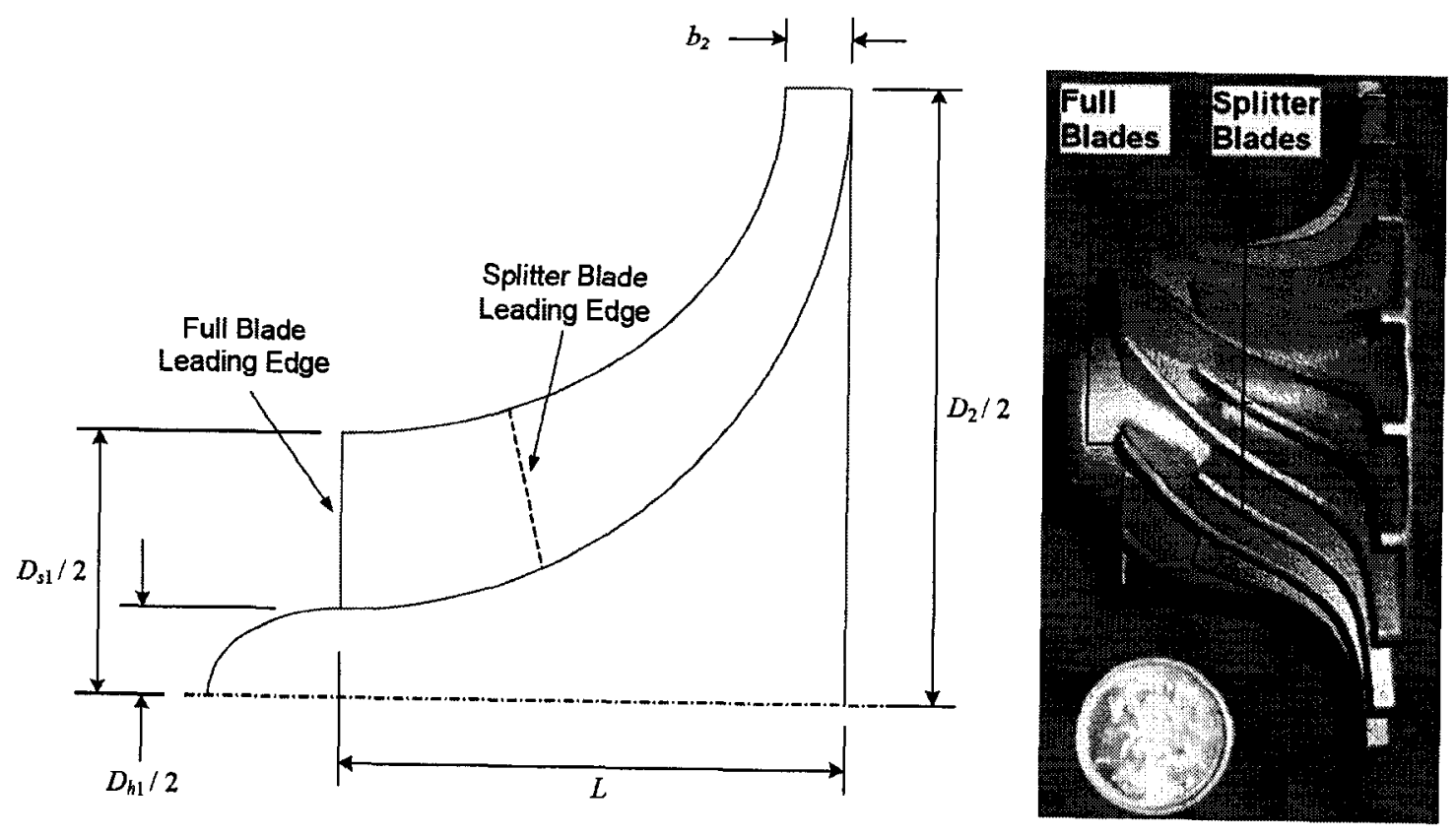

Axial Cross-Section

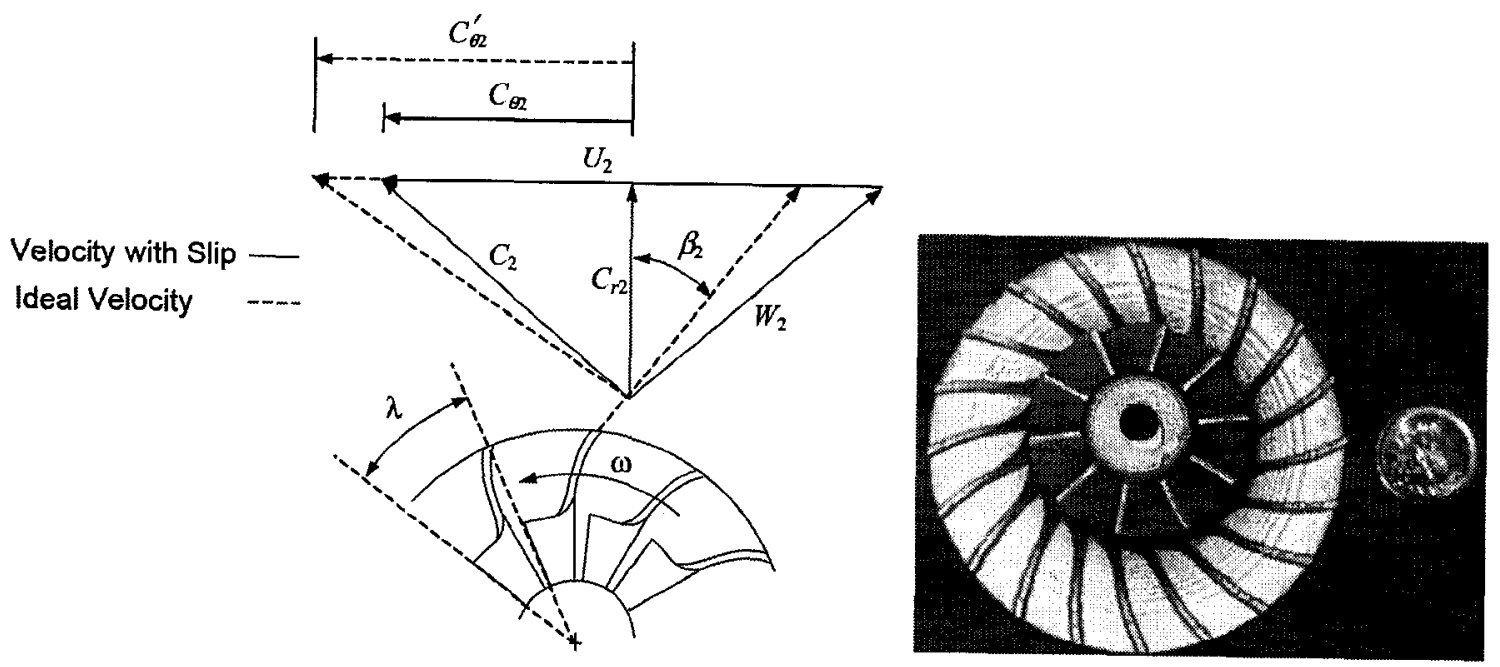

Radial Cross-Section

Figure 6.7: Rotating test rig impeller geometry and velocity triangles 


\begin{tabular}{|c|l|c|c|}
\hline & & \multicolumn{2}{|c|}{ Dimension } \\
\hline Symbol & Description & $\begin{array}{c}\text { Original Design } \\
\text { (Charest) }\end{array}$ & $\begin{array}{c}\text { Current Design } \\
\text { (Test Rig) }\end{array}$ \\
\hline$Z$ & Number of Full Blades & 19 & 10 \\
\hline$Z_{s}$ & Number of Splitter Blades & 19 & 10 \\
\hline$t$ & Impeller Blade Thickness & $0.5 \mathrm{~mm}$ & $1.25 \mathrm{~mm}$ \\
\hline$D$ & Impeller Tip Diameter & $77 \mathrm{~mm}$ & $77 \mathrm{~mm}$ \\
\hline$b_{2}$ & Impeller Tip Vane Height & $3 \mathrm{~mm}$ & $3 \mathrm{~mm}$ \\
\hline$D_{s 1}$ & Inlet Shroud Diameter & $39 \mathrm{~mm}$ & $39 \mathrm{~mm}$ \\
\hline$D_{h 1}$ & Inlet Hub Diameter & $20 \mathrm{~mm}$ & $20 \mathrm{~mm}$ \\
\hline$L$ & Axial Length & $30 \mathrm{~mm}$ & $30 \mathrm{~mm}$ \\
\hline$\beta_{1}$ & Inlet Blade Angle & $52^{\circ}$ & $52^{\circ}$ \\
\hline$\beta_{2}$ & Outlet Blade Angle (Backsweep) & $25^{\circ}$ & $25^{\circ}$ \\
\hline$\lambda$ & Outlet Wrap Angle & $50^{\circ}$ & $70^{\circ}$ \\
\hline
\end{tabular}

Table 6.2: Summary of original (Charest, 2003) and rotating test rig impeller geometries

It should be noted that although the $70^{\circ}$ wrap angle curve from Figure 6.6 represents the test rig impeller geometry, there are differences in the peak efficiencies shown in Figure 6.6 and given in Table 6.3. These differences are due to the difference in the wall surface roughness specified in BladeGen+. The efficiencies seen in Figure 6.6 were generated with zero surface roughness, or 'smooth' walls. The test rig impeller efficiency seen in Table 6.3 is predicted later as described in Section 6.2.2. In Section 6.2.2 the wall roughness specified in BladeGen+ was changed to $0.002 \mathrm{~mm}$. This roughness is expected from the investment casting process used to manufacture the impeller and should increase the accuracy of the corresponding predictions. 


\begin{tabular}{|c|l|c|c|c|}
\hline Symbol & Parameter & $\begin{array}{c}\text { Design } \\
\text { Requirement } \\
\text { (RZEGT) }\end{array}$ & $\begin{array}{c}\text { Original } \\
\text { Design } \\
\text { (Charest) }\end{array}$ & $\begin{array}{c}\text { Current } \\
\text { Design } \\
\text { (Test Rig) }\end{array}$ \\
\hline$\dot{m}$ & Mass Flow Rate $\mathrm{CO}_{2}$ & $0.444 \mathrm{~kg} / \mathrm{s}$ & $0.447 \mathrm{~kg} / \mathrm{s}$ & $0.444 \mathrm{~kg} / \mathrm{s}$ \\
\hline$P R$ & Total Pressure Ratio & 8.0 & 9.03 & 8.05 \\
\hline$\eta$ & Isentropic Efficiency & $86.3 \%$ & $87.5 \%$ & $85.4 \%$ \\
\hline$T$ & Power Input & $73.7 \mathrm{~kW}$ & $81.1 \mathrm{~kW}$ & $77.1 \mathrm{~kW}$ \\
\hline$N$ & Rotational Speed & $110,000 \mathrm{RPM}$ & $110,000 \mathrm{RPM}$ & $110,000 \mathrm{RPM}$ \\
\hline
\end{tabular}

Table 6.3: Summary of original (Charest, 2003) and rotating test rig impeller

performance parameters

\subsubsection{Detailed Aerodynamic Performance of Test Rig Impeller}

The impeller geometry was designed primarily to be manufacturable while maintaining as much of the original overall performance, at the design mass flow. The predicted performance of the test rig impeller at the original design mass flow is summarized in Table 6.3. After the geometry was finalized, the aerodynamic performance was examined for off-design rotating speeds and in more detail, again using BladeGen+. These results are presented in the current section. Even though these calculations suggested that some aerodynamic improvements might be possible, no further modifications were made to the rotating test rig impeller.

From past experience (Roberts, 2001), it was expected that it would not be possible to achieve the full design rotational speed of 110,000 RPM in the test rig. Therefore, the performance was predicted for two additional, lower rotational speeds of 85,000 and 60,000 RPM. The resulting pressure ratio and efficiency characteristics are presented in Figures 6.8 and 6.9 respectively. The reference operating point used for the more detailed aerodynamic performance is indicated on both figures. The operating point selected corresponds to the design mass flow rate specified by the Raven Zero-Emission 
Gas Turbine project and is summarized in Table 6.3. As seen in Figures 6.8 and 6.9, the reference operating point also corresponds to the lowest mass flow rate predicted by BladeGen+ at the design rotational speed. This is due to the inability of BladeGen+ to successfully solve flow fields in which there is inflow at the outlet plane of the domain. As the mass flow is reduced, at a constant speed, this inflow occurs and BladeGen + can no longer provide a converged solution. However, it is possible that a stable operating point could exist at which there is inflow at the outlet plane. It is therefore expected that the operating range of the impeller extends to lower values of mass flow for all constant speed lines.

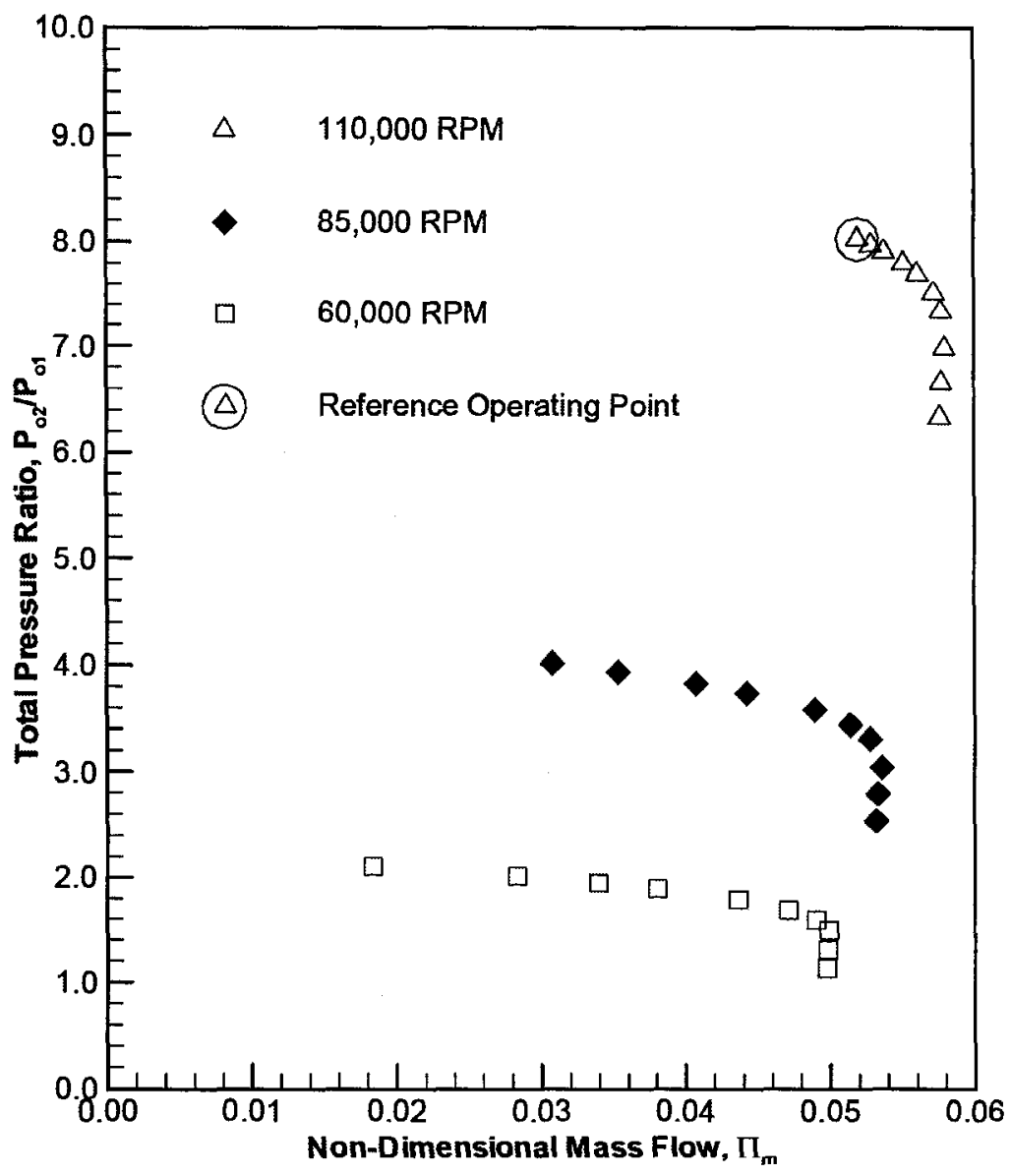

Figure 6.8: Total pressure ratio characteristic of the test rig impeller predicted by CFX-BladeGen+ 


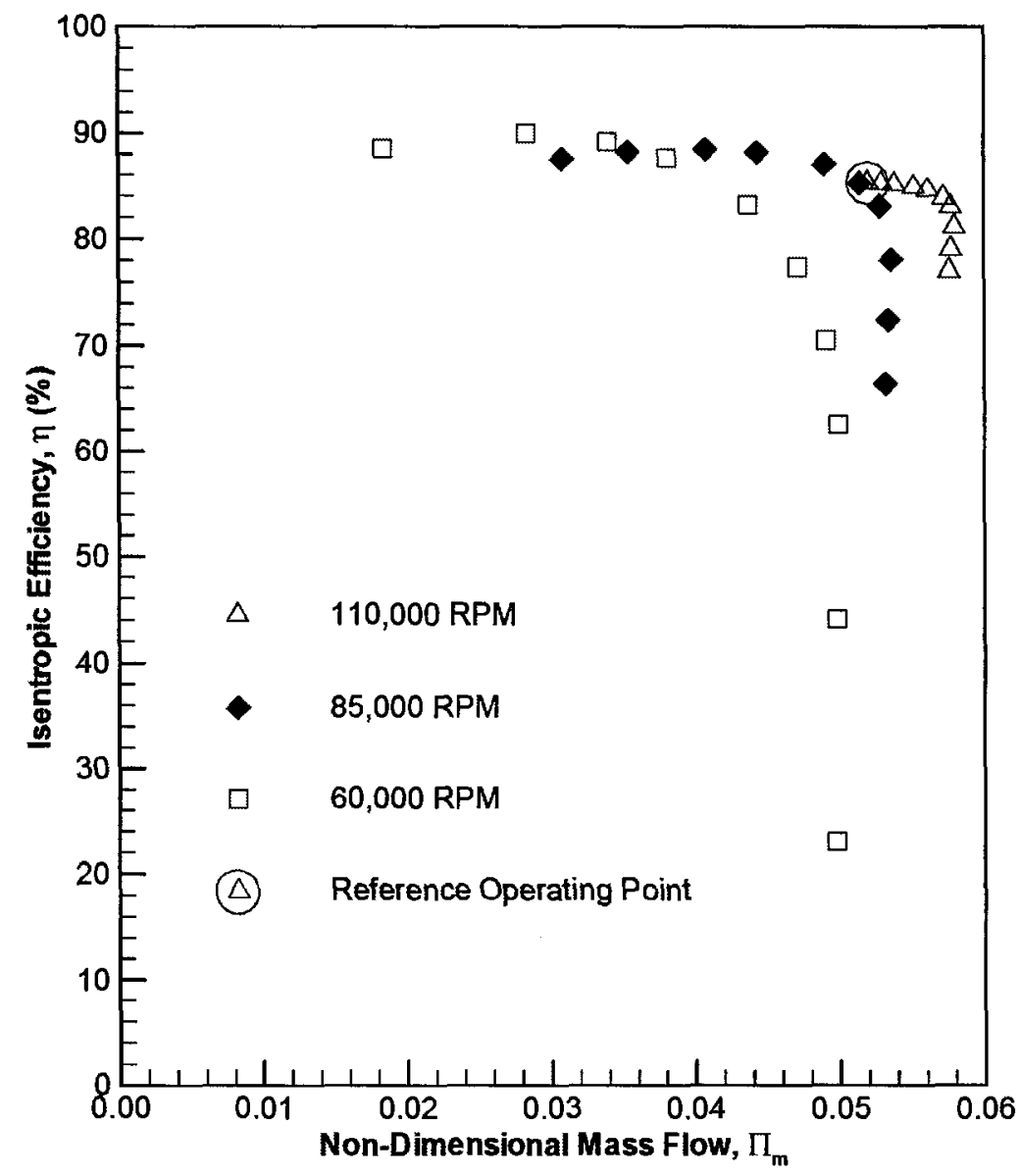

Figure 6.9: Isentropic efficiency characteristic of the test rig impeller predicted by CFX-BladeGent

Although it is expected that the mass flow range of the impeller is wider than that predicted by BladeGen+, the narrow operating range at the design speed of 110,000 RPM further illustrates that the current geometry is unsuitable for the Raven Zero-Emission Gas Turbine project. This is due to the difficulties with turbomachinery matching and poor off-design performance that would be expected from an impeller with a narrow operating range. Since the scope of this research pertains only to the performance and testing of the test rig impeller this is of minor concern provided this trait is understood by the future test engineer. As mentioned, due to power limitations of the existing rotating test rig, it is expected that testing at the design speed will not be possible. As illustrated 
by both Figures 6.8 and 6.9 the operating mass flow range is wider at lower rotational speeds, and in combination with reduced power requirements, will allow future experimental testing at reduced rotational speeds.

Figure 6.9 also shows an increased peak efficiency at lower rotational speeds. This is partly the result of decreased flow velocities because total pressure losses scale on the velocity squared. The mass-averaged inlet relative Mach numbers increase from approximately 0.40 to 0.80 from the lowest to the highest rotational speeds. In addition, the maximum relative Mach numbers seen near the shroud at the leading edge are approximately $0.65,0.95$ and 1.35 for $60,000,85,000$ and 110,000 RPM respectively at the points of maximum efficiency. Therefore, the loss in efficiency at increased rotational speeds is also partly the result of increased shock losses.

As mentioned previously, BladeGent was used to provide more detailed aerodynamic predictions at the reference operating point. These include contour plots of Mach number and total pressure. The contours of relative Mach number at $10 \%, 50 \%$ and $90 \%$ span are shown in Figure 6.10. As the span increases the surface created approaches the tips of the blades. In this instance, the tip clearance is zero and the blade tips are at $100 \%$ span, which corresponds to the shroud surface. When examining an impeller there are two frames of reference used to describe fluid properties. The absolute reference frame is stationary and flow quantities in that frame of reference are specified as absolute values. The relative reference frame rotates with the machine. Thus a relative fluid property could only be measured with a conventional sensor if it were rotated with the impeller.

It is evident from Figure 6.10 that there is a region of low velocity fluid near the trailing edge on the pressure side near the shroud surface. This indicates a degree of diffusion within the impeller but is not necessarily evidence of increased pressure losses. Of more concern would be a large region of low velocity fluid on the suction surface as 
this may lead to flow separation, and its resultant total pressure losses, if a sufficient adverse pressure gradient was present. Although there does not seem to be any presence of flow separation near the trailing edge the simple turbulence model used in BladeGen+ may not give reliable predictions for separation. Also evident from Figure 6.10 is an over speed at the blade leading edge that increases in magnitude towards the shroud. This is a result of approximately $6^{\circ}$ of positive incidence across the span. The over speed is highest near the shroud due to the increased radius and the corresponding higher blade speed. As seen, the resulting local Mach number reaches a maximum of approximately 1.35 at $90 \%$ span but only about 0.80 at $10 \%$ span.

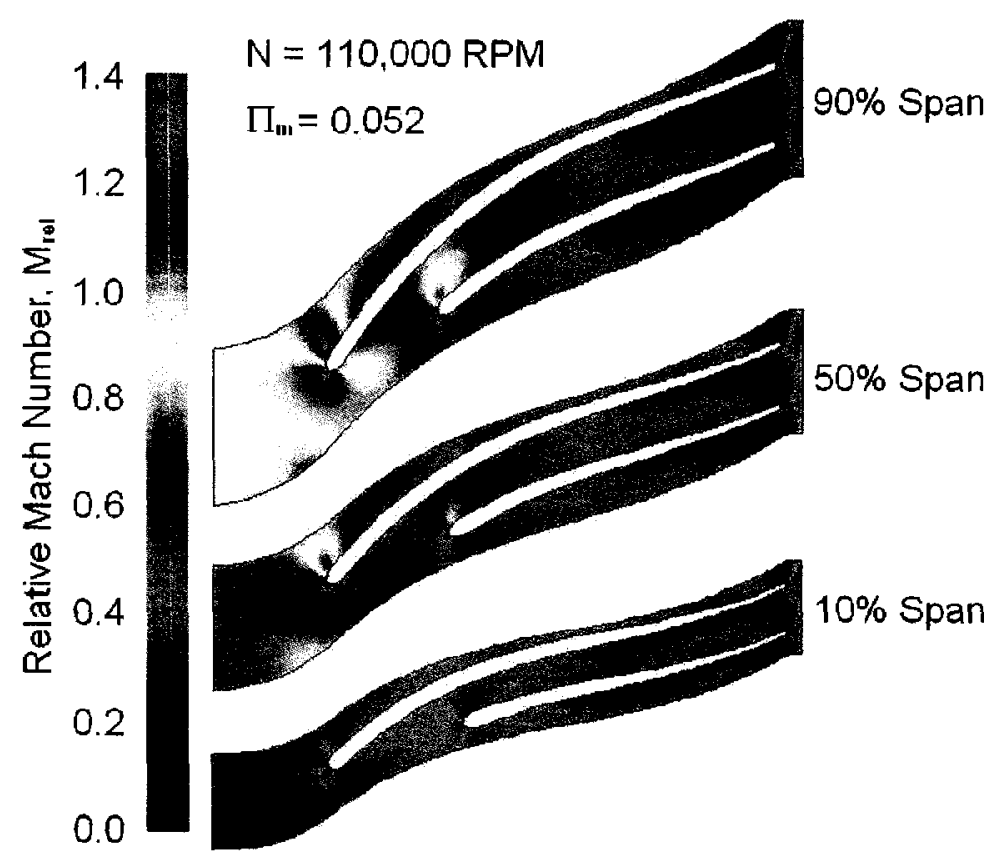

Figure 6.10: Contours of relative Mach number for the test rig impeller predicted by CFX-BladeGen+

The local supersonic Mach numbers seen in Figure 6.10 indicate that a shock wave should be present. The shock could potentially cause flow separation creating a shock-induced separation bubble. However, the computational grids created by BladeGen+ are likely unable to resolve the often small separation bubble that would be 
formed. Adding to the uncertainty about the presence of a shock-induced separation bubble is the small size and low resolution of the contour plots generated by BladeGen+.

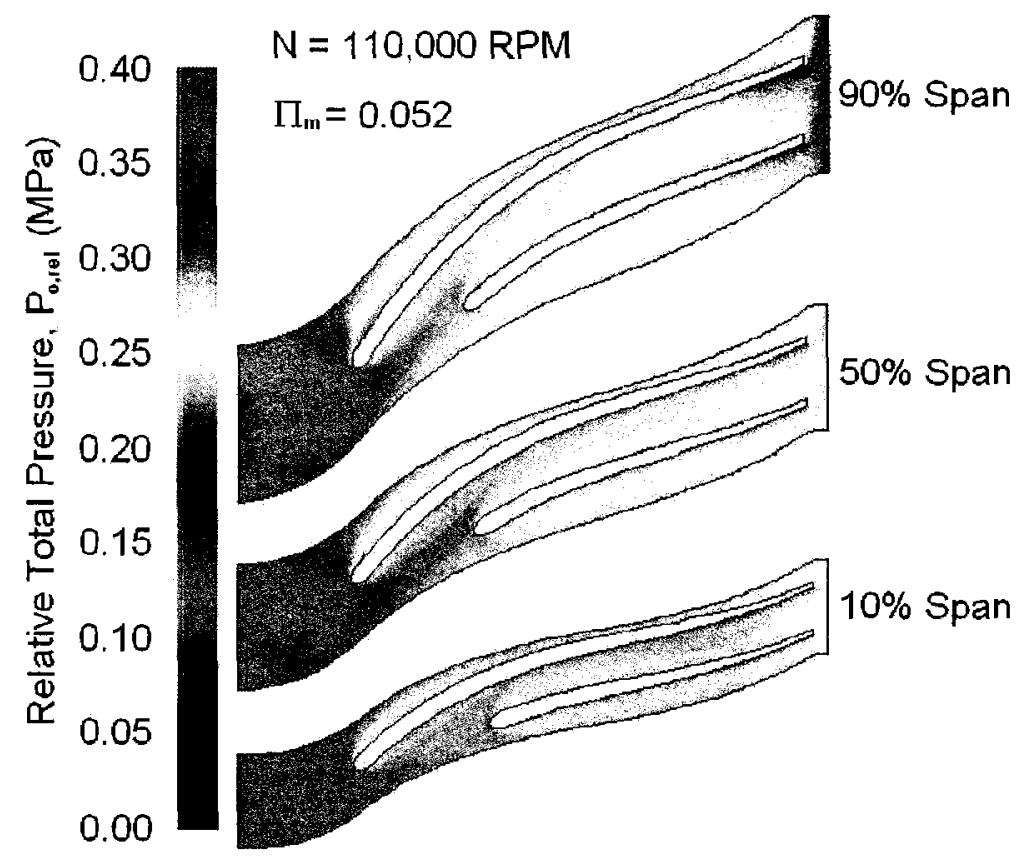

Figure 6.11: Contours of relative total pressure for the test rig impeller predicted by CFX-BladeGen+

Figure 6.11 shows the contours of relative total pressure for the same three spanwise locations as seen in Figure 6.10. The relative total pressure contours show the boundary layer formation and resultant total pressure loss. The boundary layer thickness increases and the resulting pressure losses increase in magnitude toward the shroud. Also evident from Figure 6.11 is the rapid growth of the boundary layers, particularly at higher spans. The rapid boundary layer growth there may be indirect evidence of a shockinduced separation bubble. Nevertheless, it is expected that the total pressure losses are overestimated by BladeGen+ because of the zero-equation turbulence model used. 


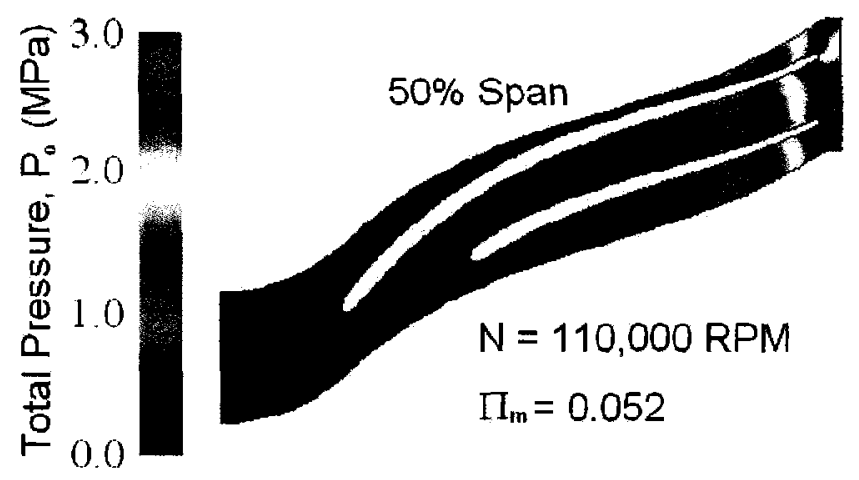

Figure 6.12: Contours of absolute total pressure for the test rig impeller predicted by CFX-BladeGen+

Figure 6.12 shows the total pressure contours in the absolute frame at mid-span. It indicates a more rapid rate of total pressure rise near the trailing edge of the impeller. From the Euler equation (Eqn. 2.5) it is expected that most of the total pressure rise would occur near the outlet where the blade speed is high.

Figure 6.13 shows the blade aerodynamic loading distribution for the main and splitter blades at mid-span. Similar to the contours of absolute total pressure, Figure 6.13 shows that the blade loading is highest beyond about $60 \%$ streamwise location. Because the loading distribution remains smooth beyond $60 \%$ it suggests that the flow remains attached to the blade surface. Also seen in Figure 6.13 is the effect of incidence at the leading edge of the main and splitter blade. The resulting over speed on the suction surface of the splitter blade interacts with the pressure surface flow of the full blade at approximately $30 \%$ streamwise location creating a small region of reversed loading. 


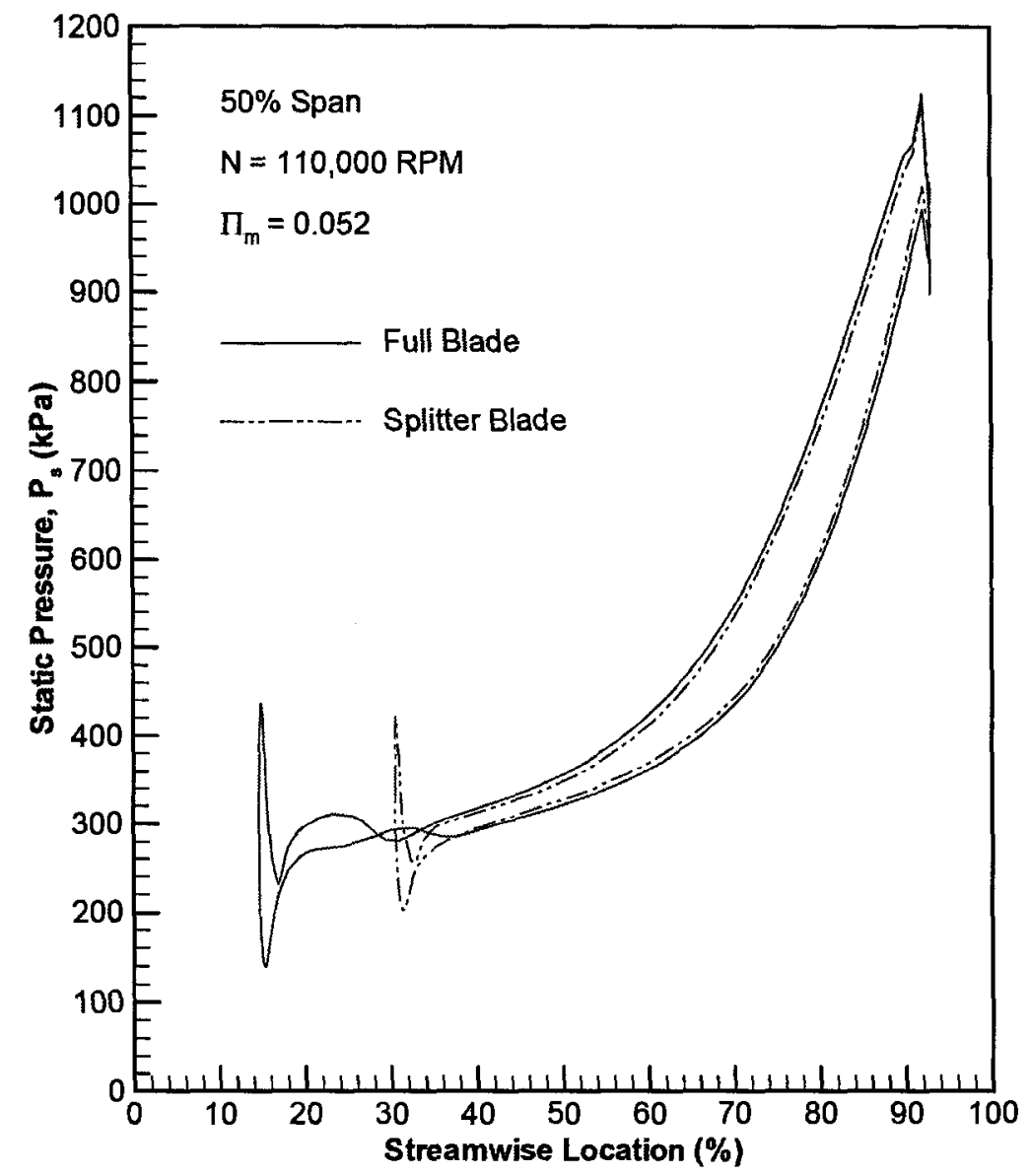

Figure 6.13: Blade loading distributions at mid-span for the test rig impeller predicted by CFX-BladeGen+

\subsection{EfFECT OF FLUID PROPERTIES ON IMPELLER OUTLET GeOMETRY}

Due to the work of authors such as Roberts (2001) we have gained a better understanding of the effect of working fluid on the performance of turbomachinery, particularly compressors. As stated earlier in Section 2.3, Roberts (2001) generated correlations (Eqns. 2.20 - 2.25) to predict the change in performance of a turbomachine based on the change in the isentropic exponent. This knowledge enables a designer to use experimental performance data obtained with one perfect gas and then predict the performance for others. Therefore, future turbomachinery designs for alternative 
working fluids could be tested in existent test facilities working with air, provided their design working fluid acts as a perfect gas. This would provide engineers with a valuable tool in the development of gas turbine cycles which use alternative working fluids. Unfortunately the understanding of working fluid effects is limited to predicting the performance of already existing machines. In an attempt to further understand the effect of the isentropic exponent on design decisions, the rotating test rig impeller was subjected to the study detailed in this section.

To begin, BladeGen+ was used to give preliminary estimates of the impeller aerodynamic performance with air as the working fluid for comparison with those results already obtained for $\mathrm{CO}_{2}$ in Section 6.2.2. The performance predictions for the two working fluids were compared to each other and to the correlations of Roberts (2001). To isolate the effect of the working fluid on the impeller performance certain nondimensional similarity criteria must be matched in addition to the geometric similarity already implied. As described in Section 2.3, there are a total of four independent nondimensional parameters that govern the performance of a compressor for which the working fluid is a compressible perfect gas. These are the mass flow parameter $\left(\Pi_{m}\right)$, the speed parameter $\left(\Pi_{N}\right)$, the Reynolds number $\left(\operatorname{Re}_{b 2}\right)$ and the isentropic exponent $(\gamma)$. A discussion of these parameters and their importance to turbomachinery design and alternative working fluids is given in Section 2.3. Their definitions are repeated here for convenience:

Mass Flow Parameter:

$$
\Pi_{m}=\frac{\dot{m} \sqrt{T_{01}}}{P_{01}}\left(\frac{1}{D_{2}{ }^{2}} \sqrt{\frac{R}{\gamma}}\right)
$$

Speed Parameter:

$$
\Pi_{N}=\frac{N}{\sqrt{T_{01}}}\left(\frac{D_{2}}{\sqrt{भ R}}\right)
$$


Reynolds Number:

$$
\operatorname{Re}_{b 2}=\frac{\rho_{01} U_{2} b_{2}}{\mu}
$$

Isentropic Exponent:

$$
\gamma=\frac{c_{p}}{c_{v}}
$$

To obtain true non-dimensionality the rotational speed $(N)$ must be specified in radians per second. However, in practice the rotational speed is often specified in RPM for convenience. With all four independent non-dimensional parameters matched all dependent parameters, such as the total pressure ratio and isentropic efficiency are also matched. By changing the working fluid from $\mathrm{CO}_{2}$ to air there is an inherent change in $\gamma$ from about 1.29 to 1.4 . It is therefore not possible to maintain complete similarity for the same impeller in the two working fluids. Thus the change in working fluid may result in a change in the impeller total pressure rise and efficiency at the same values of $\Pi_{m}, \Pi_{N}$ and $R e_{b 2}$. To investigate the ability to compensate for the effect of the isentropic exponent on the impeller performance by changing the geometry, a number of new compressors were designed to compress air.

The non-dimensional characteristics of the test rig impeller predicted by BladeGen+ are shown in Figures 6.14 and 6.15 for air and $\mathrm{CO}_{2}$, at a constant nondimensional speed of 17.23 . This corresponds to approximately 60,000 RPM for $\mathrm{CO}_{2}$ and 77,000 RPM for air. The solid line represents the performance in air predicted by the correlations of Roberts (2001), based on the BladeGen+ predicted performance in $\mathrm{CO}_{2}$. Further analysis of the reference operating points is performed later in this section. It should be noted that the current results were generated at an off-design non-dimensional speed because it provides a wider range of flow rates than the design speed and more clearly illustrates the effects of the isentropic exponent. The reference operating points 
shown in Figures 6.14 and 6.15 were selected to investigate the test rig impeller performance in $\mathrm{CO}_{2}$ and air in more detail and were chosen to have the same nondimensional speed parameter, flow parameter, Reynolds number and pressure ratio. To match the Reynolds number for both working fluids the inlet total pressure was varied.

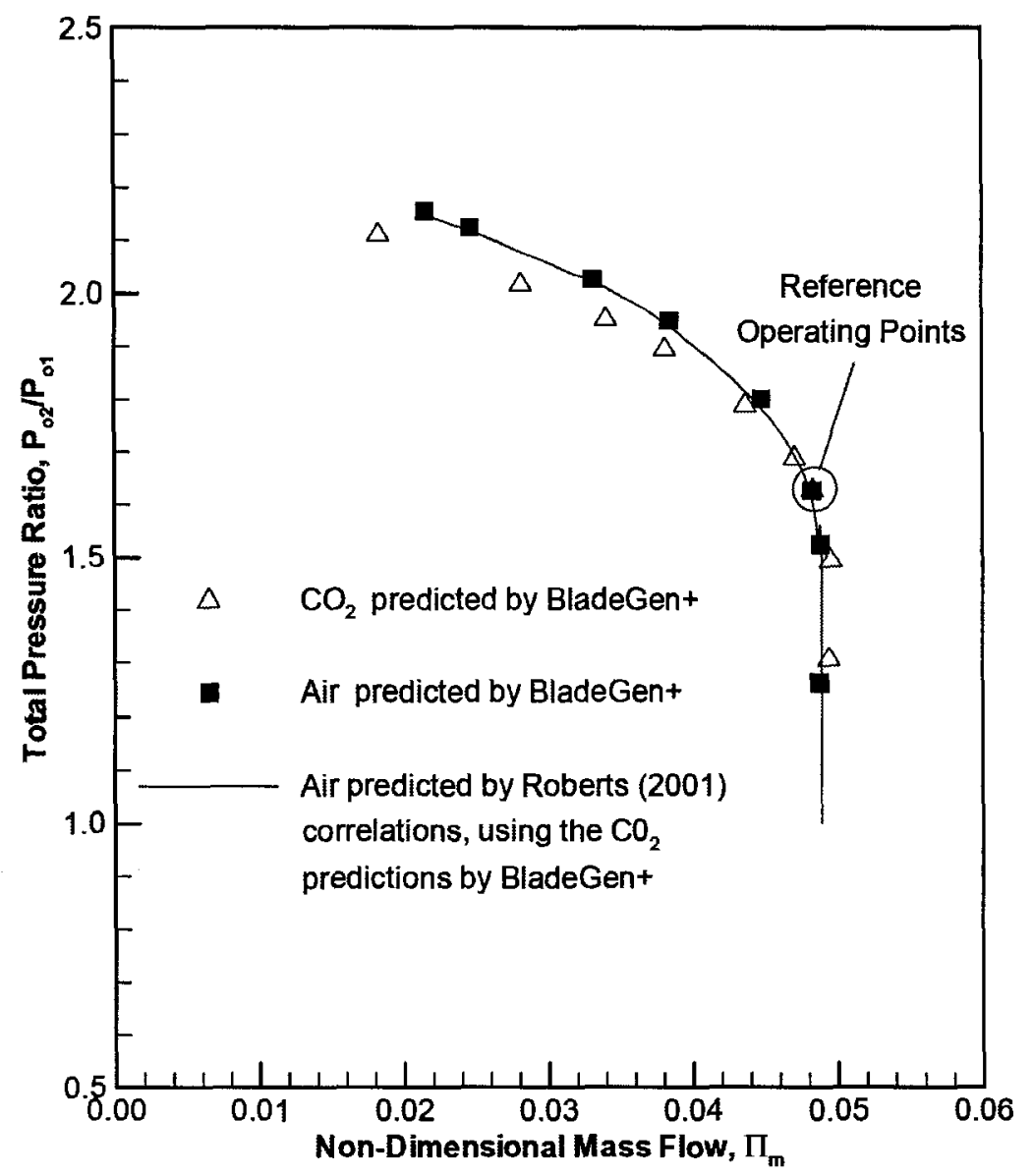

Figure 6.14: Total pressure ratio characteristic for air and $\mathrm{CO}_{2}$ predicted by CFX-BladeGent and Roberts (2001) correlations for the test rig impeller

As seen in Figures 6.14, the total pressure rise is higher for air $(\gamma=1.4)$ than for $\mathrm{CO}_{2}(\gamma=1.29)$ at a given, unchoked non-dimensional mass flow. Because the value of the mass flow parameter at choking is increased for a decreasing isentropic exponent, the constant speed lines for air and $\mathrm{CO}_{2}$ cross near the choking mass flow. At non- 
dimensional mass flows greater than the coincident point the total pressure rise is lower for air than $\mathrm{CO}_{2}$. As seen in Figure 6.15 the isentropic efficiency is higher for a gas with lower isentropic exponent. All trends are illustrated by both the BladeGen+ results and the correlations of Roberts (2001). As was the case in Roberts (2001), the correlations show better agreement to the BladeGen+ predictions for pressure ratio than efficiency.

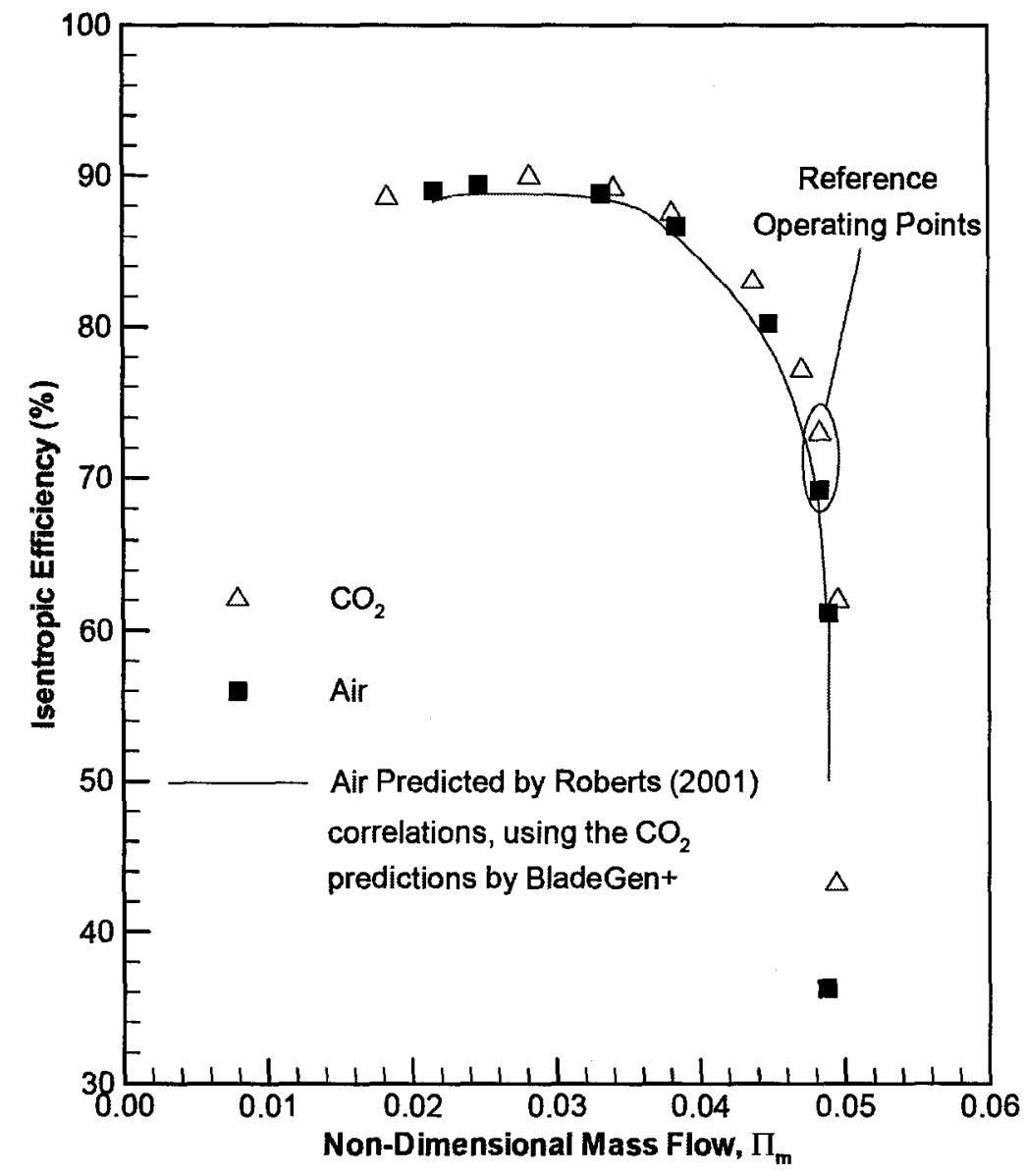

Figure 6.15: Efficiency characteristic for air and $\mathrm{CO}_{2}$ predicted by $\mathrm{CFX}$ -

BladeGen+ and Roberts (2001) correlations for the test rig impeller 
It can be shown as follows that for the same geometry the inlet Mach numbers and flow angles for two different gasses will be the same if the non-dimensional mass flow and speed parameters are matched:

Mass Flow Parameter, $\Pi_{m}$ :

$$
\left.\frac{\dot{m} \sqrt{T_{01}}}{P_{01}}\left(\frac{1}{D^{2}} \sqrt{\frac{R}{\gamma}}\right)\right|_{\text {Gas } 1}=\left.\frac{\dot{m} \sqrt{T_{01}}}{P_{01}}\left(\frac{1}{D^{2}} \sqrt{\frac{R}{\gamma}}\right)\right|_{\text {Gas } 2}
$$

where, $\dot{m}=\rho_{01} A C_{x 1}$ and $\rho_{01}=\frac{P_{01}}{R T_{01}}$

$$
\text { therefore, }\left.\frac{C_{x 1}}{\sqrt{\mathscr{R} T_{01}}}\right|_{\text {Gas } 1}=\left.\frac{C_{x 1}}{\sqrt{भ R T_{01}}}\right|_{\text {Gas } 2} \text { or }\left.M_{x 1}\right|_{G a s 1}=\left.M_{x 1}\right|_{\text {Gas2 }}
$$

\section{Speed Parameter, $\Pi_{\underline{N}}$ :}

$$
\left.\frac{N}{\sqrt{T_{01}}}\left(\frac{D}{\sqrt{\mathscr{R}}}\right)\right|_{\text {Gas } 1}=\left.\frac{N}{\sqrt{T_{01}}}\left(\frac{D}{\sqrt{\mathscr{R}}}\right)\right|_{\text {Gas } 2}
$$

since, $U=N\left(\frac{D}{2}\right)$ when $N$ is expressed in radians per second

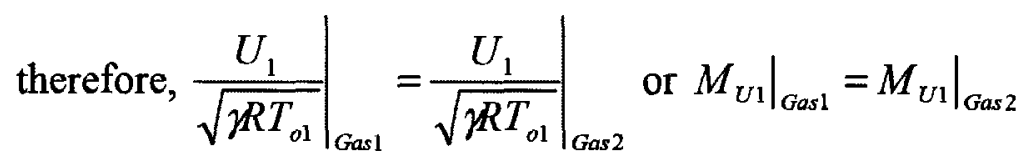

With two inlet Mach numbers equal the inlet velocity triangles are therefore matched between the working fluids when expressed non-dimensionally as Mach 
numbers. When expressed in terms of absolute magnitudes the velocity triangles are similar, and have the same flow angles, but they may have different magnitudes. Table 6.4 summarizes the performance parameters at the reference operating points for air and $\mathrm{CO}_{2}$. Recall that the reference operating points were selected by matching nondimensional speed, flow rate, Reynolds number and pressure ratio.

\begin{tabular}{|c|l|c|c|}
\hline Symbol & Description & $\mathbf{C O}_{2}$ & Air \\
\hline$\Pi_{N}$ & Non-Dimensional Speed & 17.23 & 17.23 \\
\hline$\Pi_{m}$ & Non-Dimensional Mass Flow & 0.04760 & 0.04753 \\
\hline$P R$ & Total Pressure Ratio & 1.673 & 1.668 \\
\hline$\eta$ & Isentropic Efficiency & $76.3 \%$ & $72.0 \%$ \\
\hline$a$ & Speed of Sound & $278 \mathrm{~m} / \mathrm{s}$ & $360 \mathrm{~m} / \mathrm{s}$ \\
\hline$M_{1}$ & Inlet Mach Number & 0.356 & 0.357 \\
\hline$\alpha_{1}$ & Inlet Flow Angle & $0.01^{\circ}$ & $0.01^{\circ}$ \\
\hline$W_{1}$ & Inlet Relative Velocity & $134 \mathrm{~m} / \mathrm{s}$ & $171 \mathrm{~m} / \mathrm{s}$ \\
\hline$M_{2}$ & Outlet Mach Number & 0.680 & 0.674 \\
\hline$\alpha_{2}$ & Outlet Flow Angle & $61.15^{\circ}$ & $59.82^{\circ}$ \\
\hline$W_{2}$ & Outlet Relative Velocity & $121 \mathrm{~m} / \mathrm{s}$ & $160 \mathrm{~m} / \mathrm{s}$ \\
\hline
\end{tabular}

Table 6.4: Summary of performance parameters for air and $\mathrm{CO}_{2}$ as predicted by BladeGen+ at the reference operating points of the test rig impeller

As seen in Table 6.4, matching the non-dimensional speed and flow rate resulted in similar inlet velocity triangles as expected. However, the isentropic efficiency is higher for $\mathrm{CO}_{2}$ than air. With a change in the working fluid from $\mathrm{CO}_{2}$ to air there has been a corresponding change in the speed of sound, $a=\sqrt{\gamma R T}$. Thus the similar inlet and outlet velocity triangles imply a difference in the magnitudes of velocity present in the impeller. The increase in the speed of sound from $\mathrm{CO}_{2}$ to air has increased the relative velocity levels in the impeller when operating in air. It is known that the 
frictional pressure losses in an impeller vary on the relative velocity squared. The difference seen in the isentropic efficiency is therefore a result of the change in the speed of sound inherent to the change in isentropic exponent. However, the speed of sound is also a function of the gas constant $R$. It is possible that two gasses could have the same value of isentropic exponent with different gas constants. The resulting speed of sound would therefore be different for the two gasses at the same non-dimensional parameters and pressure ratio. The impeller efficiency should presumably be different for each gas. To be certain further analysis of the impeller performance in working fluids with similar isentropic exponents and different gas constants is required. Further analysis was not conducted because the scope of the current study was focussed on counteracting the change in performance resulting from the change in working fluid.

At the reference operating points the difference in the impeller efficiency with each working fluid is attributed mainly to the difference in the speed of sound. The difference in the speed of sound results in higher flow velocities within the impeller operating in air and therefore higher skin-friction pressure losses and lower efficiency. It should be possible to counteract this loss in efficiency by altering the outlet geometry of the impeller when compressing air. To achieve this several geometric parameters of the impeller were investigated to determine their influence on the impeller performance. Based on the influence of each geometric parameter a new impeller geometry was created to match the performance of the original impeller in $\mathrm{CO}_{2}$ but was designed to compress air. The reference operating points considered here were selected with matching total pressure ratios and therefore only the efficiency needs to be corrected with the geometric modifications. For a more general operating point where the change in working fluid caused both the pressure ratio and efficiency to change, it may be possible to adjust the geometry in ways such that the two compressors produce the same pressure ratio and efficiency at the same non-dimensional speed, non-dimensional mass flow rate and Reynolds number. 
The geometric parameters of the impeller that were investigated are the exit vane height $\left(b_{2}\right)$, backsweep angle $\left(\beta_{2}\right)$ and wrap angle $(\lambda)$. These parameters were selected because they do not affect the inlet geometry of the impeller and they can be modified in BladeGen with relative ease. All geometric parameters were investigated with air at the reference operating mass flow rate and rotational speed. By maintaining the mass flow rate through the impeller the variation in exit vane height will determine the radial component of velocity at the outlet $\left(C_{r 2}\right)$, from continuity

$$
\dot{m}=\rho A V=\rho_{2} C_{r 2} A_{2}=\rho_{2} C_{r 2}\left(\pi D D_{2} b_{2}\right)
$$

Thus an increase in the exit vane height will result in a decreased outlet radial velocity.

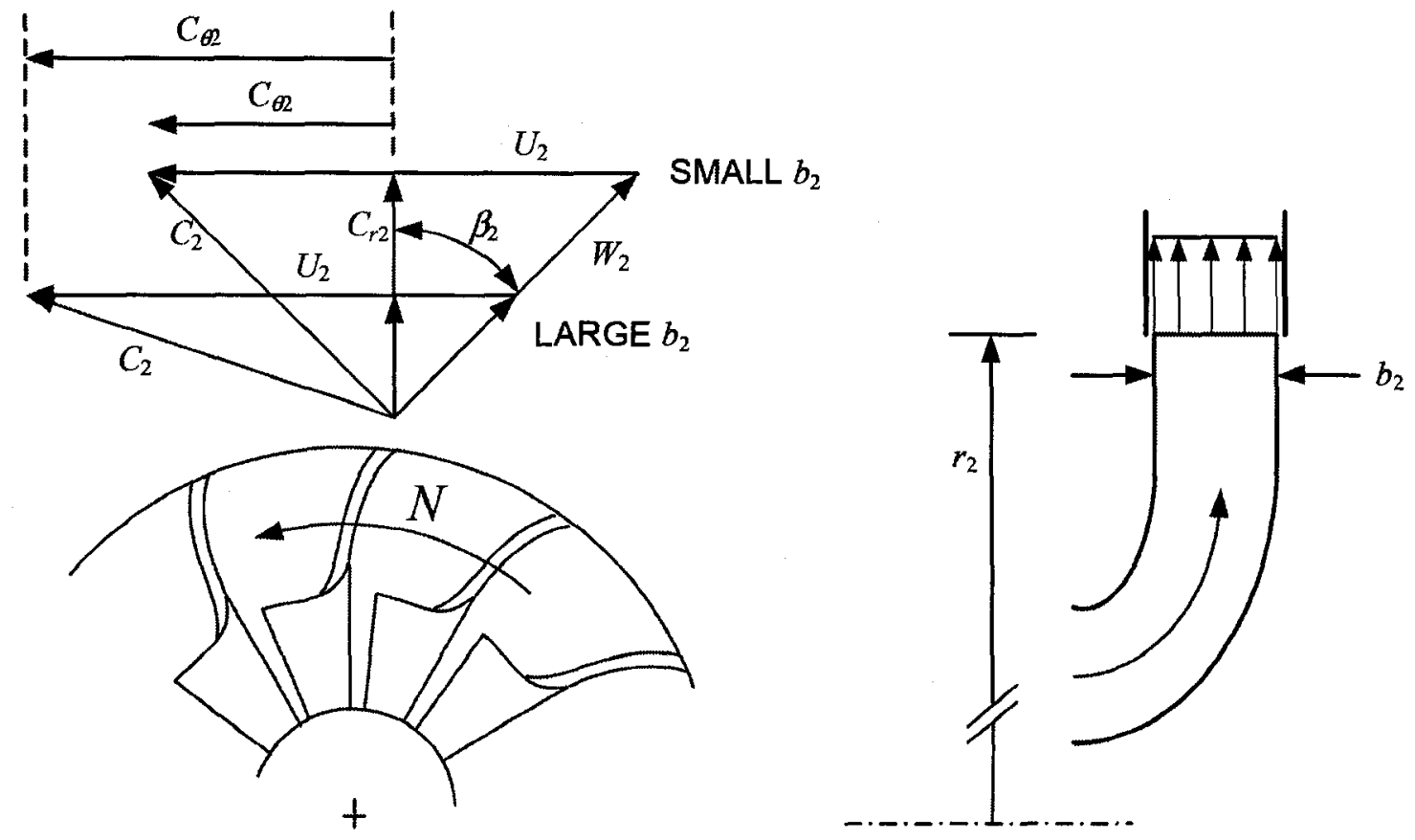

Figure 6.16: Effect of exit vane height on rotor outlet velocity triangle

Figure 6.16 shows the ideal rotor outlet velocity triangles for two radial velocities at constant blade speed $\left(U_{2}\right)$. With a constant backsweep angle $\left(\beta_{2}\right)$ the reduced radial velocity results in a lower relative velocity at the outlet $\left(W_{2}\right)$. As mentioned previously the relative velocity within the rotor will influence the skin-friction pressure losses and 
therefore the efficiency of the impeller. In addition the diffusion ratio $\left(W_{2} / W_{1}\right)$ of the impeller is a function of the outlet relative velocity. Larger diffusion occurs with a decreased outlet relative velocity and may lead to additional pressure losses if an excessive level of diffusion leading to separation is present. The reduced radial velocity has also increased the absolute tangential velocity $\left(C_{\theta 2}\right)$. From the Euler equation (Eqn. 2.5) any increase in the outlet tangential velocity will raise the ideal total pressure rise.

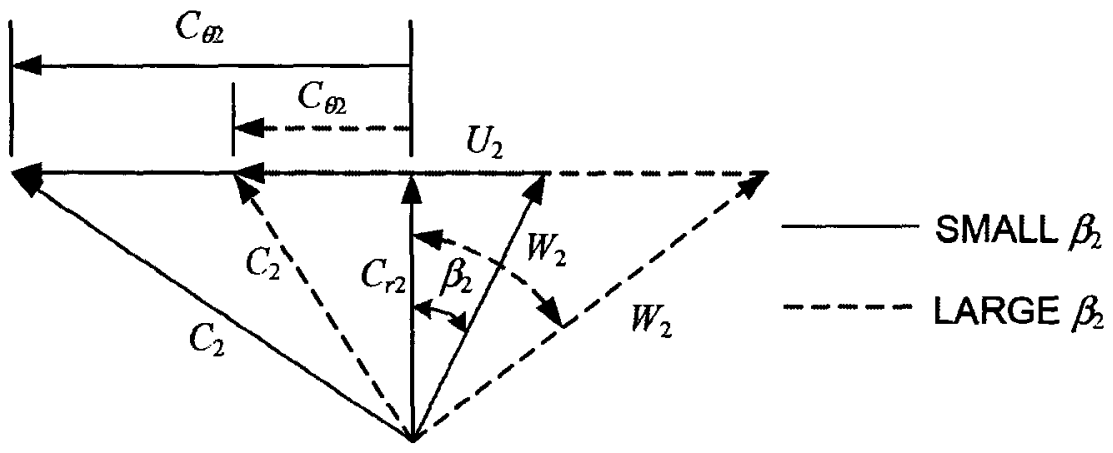

Figure 6.17: Effect of backsweep angle on rotor outlet velocity triangle

The effect of the backsweep angle on the ideal discharge velocity triangle of the impeller is shown in Figure 6.17. Again the blade speed and radial velocity are kept constant for various backsweep angles. As seen, an increased backsweep angle will reduce the tangential velocity and thus lower the ideal pressure rise. In addition, higher backswept blades will reduce the diffusion within the impeller due to the increase in the outlet relative velocity. It is known that lower levels of diffusion tend to increase the efficiency of the impeller if it reduces or eliminates separation. The increased backsweep angle also increases the outlet relative velocity. Again skin-friction losses within the impeller vary with the relative velocity. The effect of the backsweep angle on the efficiency is therefore a trade-off between diffusion levels and skin-friction pressure losses. However, changes in the flow due to the change in backsweep angle should only occur near the trailing edge. The increased skin-friction pressure losses should be small and the efficiency of the rotor will typically increase at larger backsweep angles as noted by Saravanamuttoo et al. (2001). 
The final geometric parameter investigated was the wrap angle. The wrap angle is defined as the circumferential angle between the blade leading and trailing edges. Unlike the vane height and backsweep the wrap angle has no influence on the ideal outlet velocity triangle. Instead the wrap angle affects the passage length through the impeller such that an increased wrap angle implies a longer vane passage. An increase in the vane passage length reduces the aerodynamic blade loading. Thus the wrap angle could presumably be used to reduce the number of vanes and subsequently reduce the wetted area of the rotor. In the current investigations the number of vanes was unchanged. With the same number of vanes, the longer passages should guide the flow more effectively and lower the deviation or slip at the trailing edge which would increase the pressure ratio. However, a longer vane passage also increases the wetted area of the vane which in turn increases the skin-friction total pressure losses. It is expected that an impeller with a larger wrap angle and same number of vanes will have a lower efficiency due to the increased wetted area. The effect of wrap angle on the pressure rise is dependent on the magnitude of skin-friction losses and slip. Table 6.5 summarizes the expected effect of the vane height, backsweep angle and wrap angle on the impeller total pressure ratio and efficiency.

\begin{tabular}{|c|l|c|c|c|}
\hline Symbol & Description & Change & \multicolumn{2}{|c|}{ Effect on } \\
\cline { 4 - 5 } & & Efficiency & Pressure Ratio \\
\hline$b_{2}$ & Outlet Vane Height & $\uparrow$ & $\begin{array}{c}\text { Trade-off between } \\
\text { skin-friction losses } \\
\text { and diffusion }\end{array}$ & $\uparrow$ \\
\hline$\beta_{2}$ & Backsweep Angle & $\uparrow$ & $\uparrow$ & $\downarrow$ \\
\hline$\lambda$ & Wrap Angle & $\downarrow$ & $\uparrow$ & $\begin{array}{c}\text { Trade-off between } \\
\text { skin-friction losses } \\
\text { and slip }\end{array}$ \\
\hline
\end{tabular}

Table 6.5: Expected effect of geometric parameters on impeller performance 
Table 6.5 summarizes the expected trends in the impeller performance by varying particular geometric properties. To determine the actual change in the impeller performance the geometric properties of the test rig impeller were individually varied within CFX-BladeGen. The new geometries were then solved in BladeGen+ and their performance parameters are summarized in Table 6.6 at the reference operating speed and mass flow using air as the working fluid. All geometric properties and velocities are specified at the rotor outlet. The geometric modifications are compared against the original test rig impeller to show the actual effect of each parameter on the impeller performance.

Table 6.6 summarizes the effect of exit vane height, backsweep and wrap angle on the impeller performance predicted by BladeGen+. Mostly the trends are consistent with those summarized in Table 6.5, namely an increased backsweep angle reduced the pressure ratio and increased the efficiency while a decreased wrap angle increased the pressure ratio and efficiency. The increased pressure ratio with decreased wrap angle suggests that the skin-friction pressure losses have a greater impact on the pressure rise than the slip at the operating point investigated. In addition, an increased exit vane height has increased the pressure ratio as suggested by Table 6.5 .

Also seen in Table 6.6 is an increase in the efficiency with a larger exit vane height. This is a result of the diffusion ratio or de Haller number of the impeller. Several authors including Aungier (2000), Wilson and Korakianitis (1998), Japikse and Baines (1997) and Saravanamuttoo et al. (2001) present minimum values of the de Haller number for satisfactory compressor performance. Although there is some variation in the recommendations of each author the minimum de Haller number is suggested as approximately 0.75 . As seen in Table 6.6 the de Haller number of the test rig impeller at the reference operating point is considerably higher than the suggested minimum value. Even with an increased exit vane height the de Haller number is still well above the suggested limited. The level of diffusion within the impeller would suggest that there is 
not a significant increase in separation and thus total pressure losses when increasing the vane height from $3.0 \mathrm{~mm}$ to $3.5 \mathrm{~mm}$. Because increased diffusion will only reduce the impeller efficiency when it increases or causes separation, the reduced relative velocities and subsequent decreased total pressure losses have resulted in an increased efficiency.

\begin{tabular}{|c|l|c|c|c|c|}
\hline Symbol & Description & $\begin{array}{c}\text { Test Rig } \\
\text { Impeller }\end{array}$ & $\begin{array}{c}\text { Increased } \\
\mathbf{b}_{2}\end{array}$ & $\begin{array}{c}\text { Increased } \\
\boldsymbol{\beta}_{2}\end{array}$ & $\begin{array}{c}\text { Decreased } \\
\lambda\end{array}$ \\
\hline$b_{2}$ & Vane Height & $3.0 \mathrm{~mm}$ & $3.5 \mathrm{~mm}$ & $3.0 \mathrm{~mm}$ & $3.0 \mathrm{~mm}$ \\
\hline$\beta_{2}$ & Backsweep Angle & $25^{\circ}$ & $25^{\circ}$ & $30^{\circ}$ & $25^{\circ}$ \\
\hline$\lambda$ & Wrap Angle & $70^{\circ}$ & $70^{\circ}$ & $70^{\circ}$ & $65^{\circ}$ \\
\hline$P R$ & Total Pressure Ratio & 1.63 & 1.71 & 1.58 & 1.69 \\
\hline$\eta$ & Isentropic Efficiency & $69.2 \%$ & $72.1 \%$ & $70.2 \%$ & $73.6 \%$ \\
\hline$C_{\theta 2}$ & $\begin{array}{l}\text { Absolute Tangential } \\
\text { Velocity }\end{array}$ & $207 \mathrm{~m} / \mathrm{s}$ & $219 \mathrm{~m} / \mathrm{s}$ & $193 \mathrm{~m} / \mathrm{s}$ & $210 \mathrm{~m} / \mathrm{s}$ \\
\hline$\sigma$ & Slip Factor & 0.827 & 0.839 & 0.815 & 0.832 \\
\hline$W_{2}$ & Relative Velocity & $165 \mathrm{~m} / \mathrm{s}$ & $140 \mathrm{~m} / \mathrm{s}$ & $174 \mathrm{~m} / \mathrm{s}$ & $160 \mathrm{~m} / \mathrm{s}$ \\
\hline$W_{2} / W_{1}$ & De Haller Number & 0.956 & 0.812 & 1.01 & 0.925 \\
\hline
\end{tabular}

Table 6.6: CFX-BladeGen+ performance predictions for various geometric modifications

Using Table 6.6 as a guide a new geometry was designed to try to obtain an impeller that gave the same performance as the original impeller in $\mathrm{CO}_{2}$. Table 6.7 summarizes the geometric modifications made and the resulting performance in air compared to the original test rig impeller performance. As seen the simple geometric modifications have successfully counteracted the change in performance resulting from the change in working fluid. 


\begin{tabular}{|c|l|c|c|c|}
\hline Symbol & Description & \multicolumn{2}{|c|}{ Test Rig Impeller } & Modified \\
\hline$b_{2}$ & Vane Height & \multicolumn{2}{|c|}{$3.0 \mathrm{~mm}$} & $3.0 \mathrm{~mm}$ \\
\hline$\beta_{2}$ & Backsweep Angle & \multicolumn{2}{|c|}{$25^{\circ}$} & $30^{\circ}$ \\
\hline$\lambda$ & Wrap Angle & \multicolumn{2}{|c|}{$70^{\circ}$} & $65^{\circ}$ \\
\hline & Working Fluid & $\mathrm{CO}_{2}$ & Air & Air \\
\hline$\Pi_{N}$ & Non-Dimensional Speed & 17.23 & 17.23 & 17.23 \\
\hline$\Pi_{m}$ & Non-Dimensional Mass Flow & 0.04834 & 0.04831 & 0.04831 \\
\hline$P R$ & Total Pressure Ratio & 1.63 & 1.63 & 1.63 \\
\hline$\eta$ & Isentropic Efficiency & $72.9 \%$ & $69.2 \%$ & $73.5 \%$ \\
\hline
\end{tabular}

Table 6.7: Geometric modifications required to counteract the change in performance due to the change in working fluid

As mentioned previously the total pressure ratio of the test rig impeller was chosen to be equal for the two working fluids at the reference operating point. Thus the modified geometry has only counteracted the effect of the working fluid on the efficiency. However, a similar process could be applied to operating points with different pressure ratios. It should then be possible to counteract the effect of the working fluid on both the pressure ratio and efficiency using the same methodology, at least on one specific reference operating point.

The current study only investigated the geometric modifications for a single operating point. It is known from Saravanamuttoo et al. (2001) and Japikse and Baines (1997) that modifying the backsweep angle will alter the constant speed characteristic of an impeller. It is therefore expected that the constant speed operating line of the modified geometry is different from the original test rig impeller. Figure 6.18 and 6.19 show the performance characteristics of the modified geometry with air as the working fluid. The constant speed lines of the original test rig impeller in both $\mathrm{CO}_{2}$ and air are also shown. 


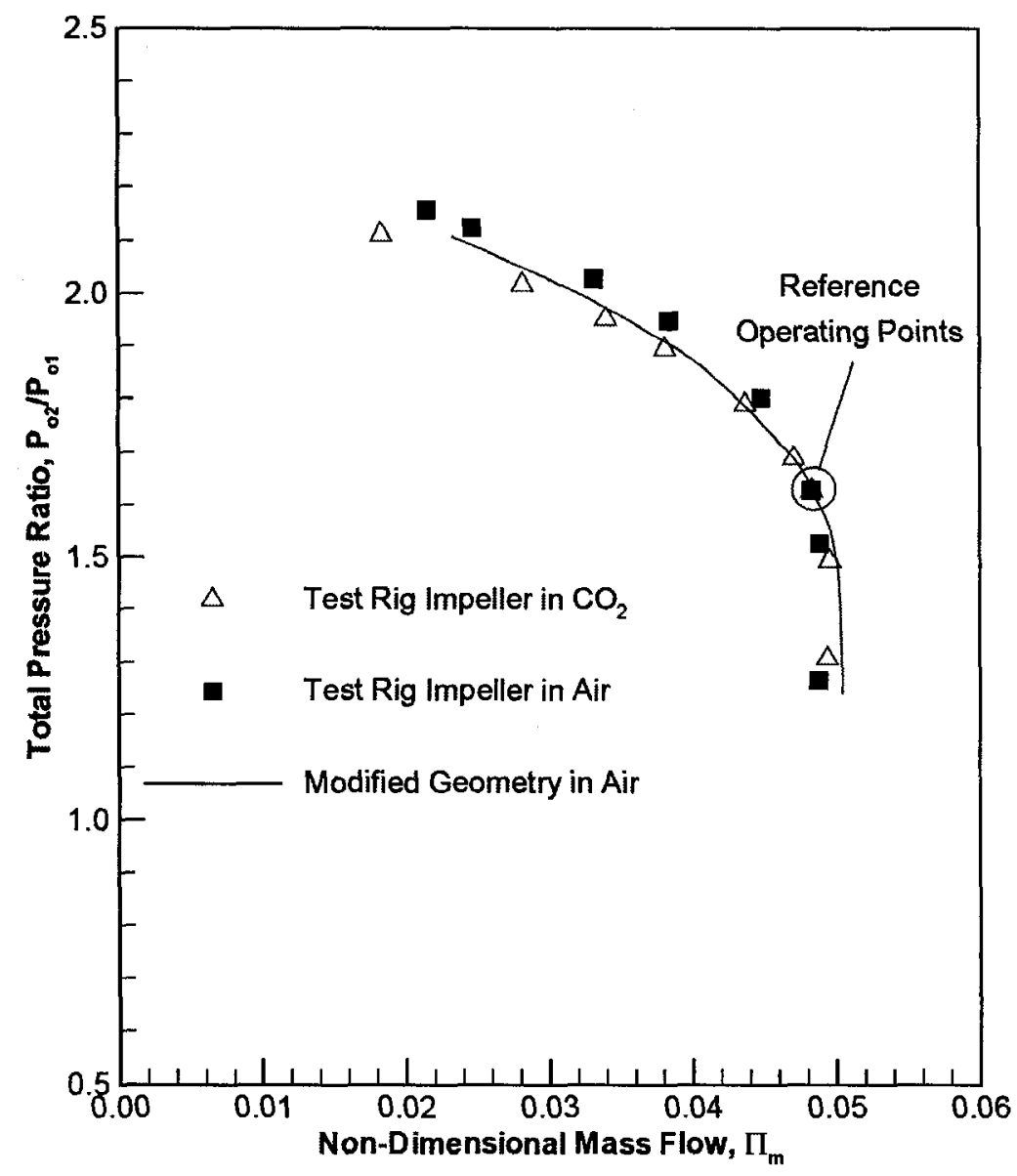

Figure 6.18: Total pressure ratio characteristic of the modified impeller geometry

As seen in Figure 6.18 the geometric modifications have only partially counteracted the effect of working fluid on the pressure rise at operating points other than the reference point. In addition the choking mass flow is larger than the original test rig impeller choking mass flow in either working fluid as seen in both Figures 6.18 and 6.19. Also shown in Figure 6.19 is that the peak efficiency of the modified geometry has not increased to match the original performance in $\mathrm{CO}_{2}$. It is therefore clear that the modifications made to counteract the influence of working fluid on the impeller performance have done so only at the reference operating point. Thus, the process used 
is not usable for counteracting the influence of working fluid on the full constant speed operating line.

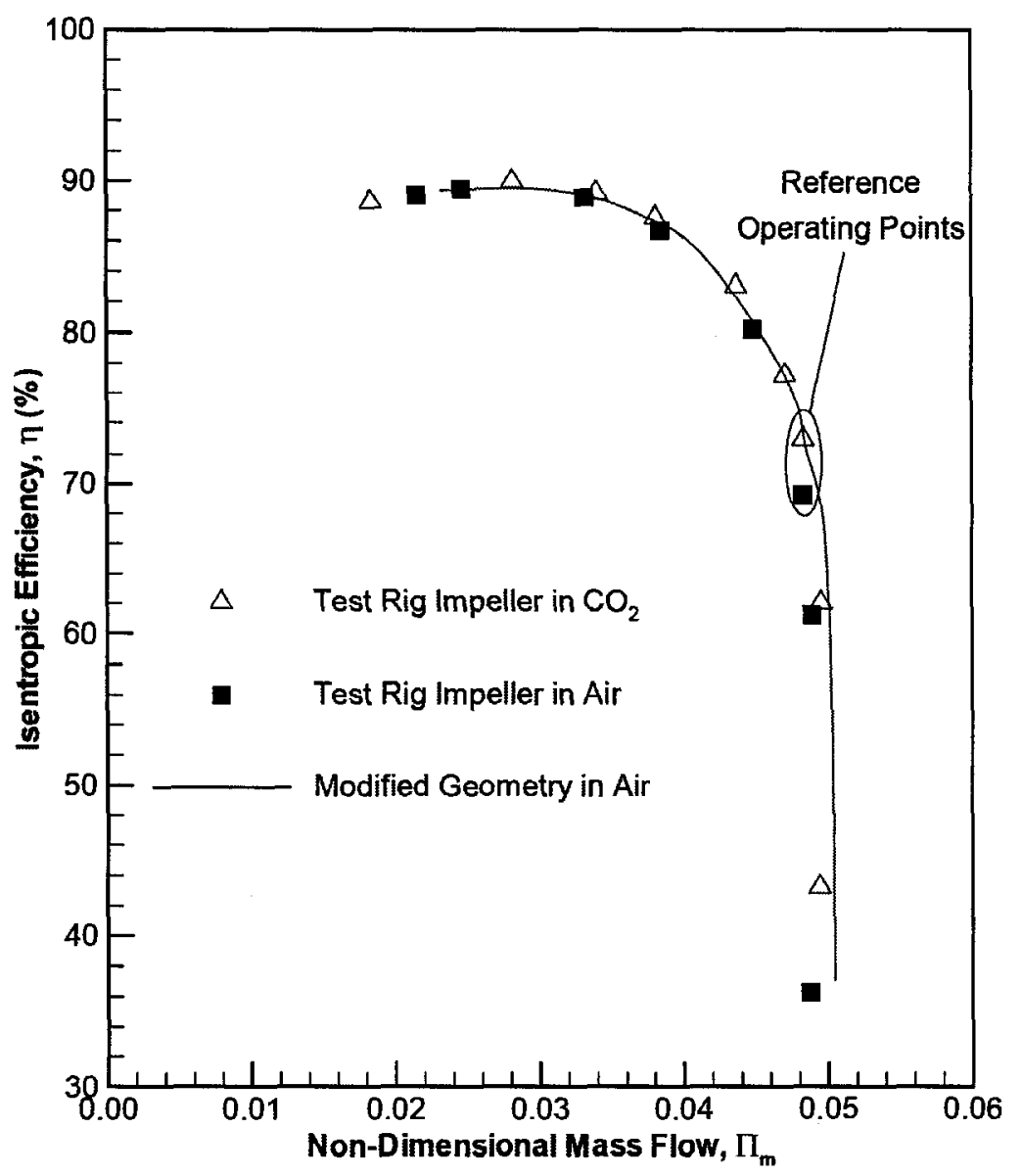

Figure 6.19: Efficiency characteristic of the modified impeller geometry 


\subsection{TURBONETICS T04E-50 COMPRESSOR RESULTS AND DISCUSSION}

\subsection{INTRODUCTION}

The original intent of this study was a detailed aerodynamic analysis of the rotating test rig compressor performance, both experimentally and computationally. Unfortunately, due to time constraints and manufacturing delays the collection of the experimental test data was not feasible. Instead, the aerodynamic analysis of the test rig compressor was limited to CFD predictions. To validate the CFD predictions an alternative impeller was needed to obtain the comparison between experimental measurements and CFD predictions for the same impeller. The Turbonetics T04E-50 compressor impeller was selected because it was previously installed in the existing rotating test rig and was ready for experimental testing. The current chapter discusses the results of both the experimental measurements and the computational predictions by ANSYS CFX for the Turbonetics T04E-50 compressor impeller.

The rotating test rig, designed by Roberts (2001), had not been used for several years. To ensure that the test rig was still capable of providing reliable test data the experimental results are compared, in Section 7.2, to those available from the manufacturer. Once the test rig was shown to provide satisfactory basic compressor performance data it was decided to broaden its capabilities. To do this new rotatable total pressure probes were installed near the impeller exit at several circumferential locations. These probes allowed more detailed flow information to be measured near the impeller exit. The effect of the new total pressure probes on the compressor performance is discussed in Section 7.3.1 and the experimental results obtained are discussed in Sections 7.3.2 to 7.3.3. Additionally, static pressure taps were added to the compressor casing to 
measure variations in the static pressure along the shroud surface and volute. These results are presented in Sections 7.3.4 and 7.3.5.

The results of the performance predictions generated by ANSYS CFX are then discussed in Section 7.4. The impeller performance characteristics, the detailed measurements made downstream of the impeller outlet, and the shroud surface static pressures are all compared to the CFD predicted values. Only the impeller performance has been predicted since the detailed geometry of the volute was not available for the T04E-50 compressor.

\subsection{RE-COMmISSIONING the TEST StAND}

The rotating test rig had been idle since the work of Roberts (2001). In addition, the turbocharger had been removed from the test stand and dismantled on several occasions. This was done to enable a three-dimensional scan of the impeller to be done, and geometric measurements made of the compressor casing. It was therefore necessary to re-commission the test stand. As part of this process, the compressor performance was measured and compared to the manufacture's data, as well as the earlier measurements by Roberts.

Figures 7.1 and 7.2 show the total pressure ratio and isentropic efficiency characteristics of the Turbonetics T04E-50 compressor as originally measured by Roberts (2001). The measured data are compared with the performance data available from the manufacturer. As can be seen, good agreement was achieved, most notably at the lowest two constant speed lines. As noted by Roberts (2001), the difference between the measured performance and the manufacturer's data at higher pressure ratios was thought to be due to normal variations in manufacturing from compressor to compressor, due to the cast volute and the expected differences in surface roughness and passage area of different machines of the same design. 


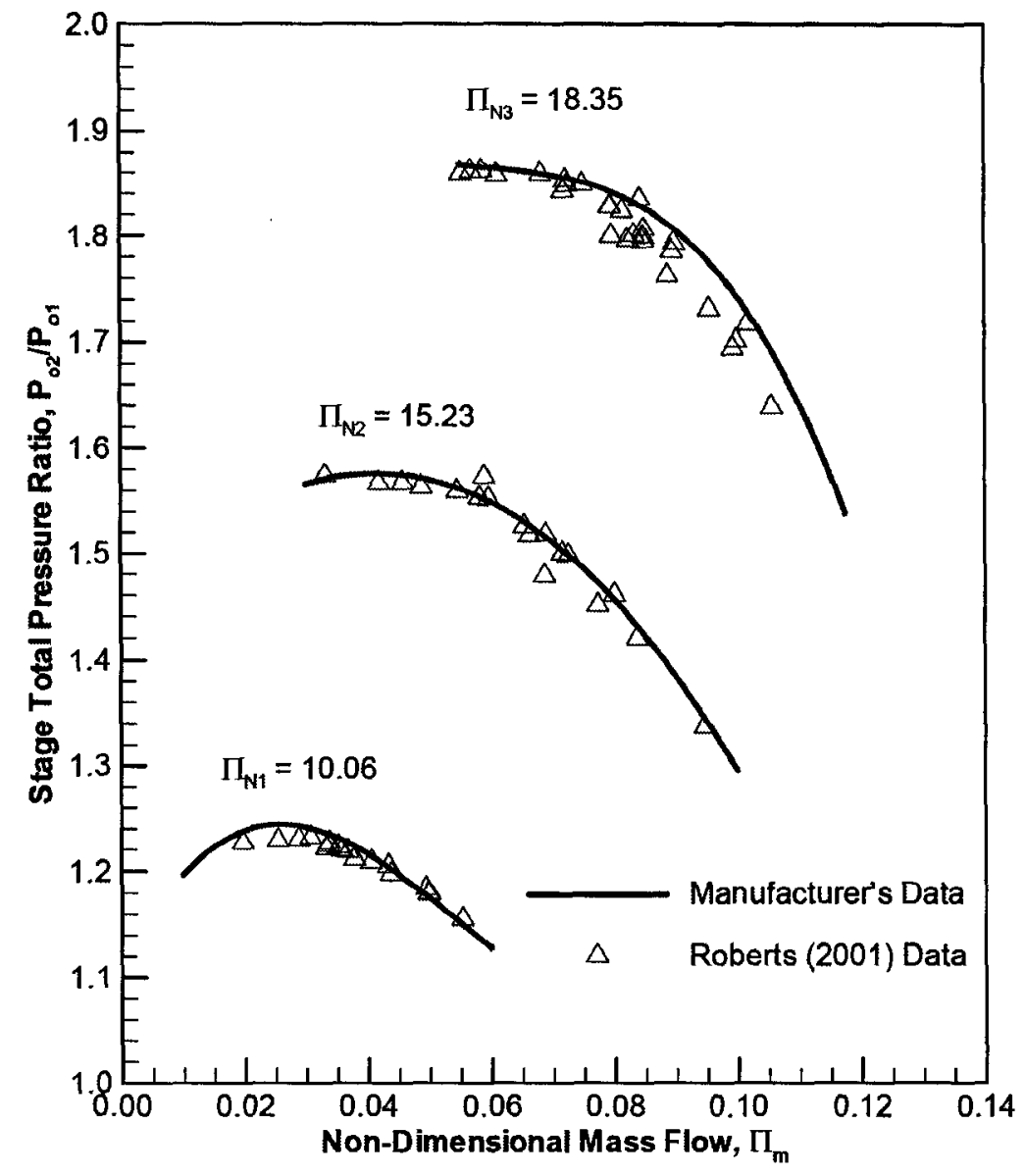

Figure 7.1: Total pressure ratio characteristic of the T04E-50 compressor originally measured by Roberts (2001)

The non-dimensional speed parameters of $10.06,15.23$ and 18.35 correspond to rotational speeds of approximately 46,000, 69,000 and 84,000 RPM respectively. Originally, Roberts (2001) had collected performance data for a fourth constant speed line at approximately 96,000 RPM. This data has been omitted from the figures since tachometer problems prevented testing at this higher speed during the current measurements.

The rotating test rig is capable of collecting performance data for working fluids other than air when operating in closed-circuit mode. However, for simplicity all test 
data in the present work was collected in open circuit configuration with air as the working fluid. The corresponding CFD predictions were also generated using only air as the working fluid.

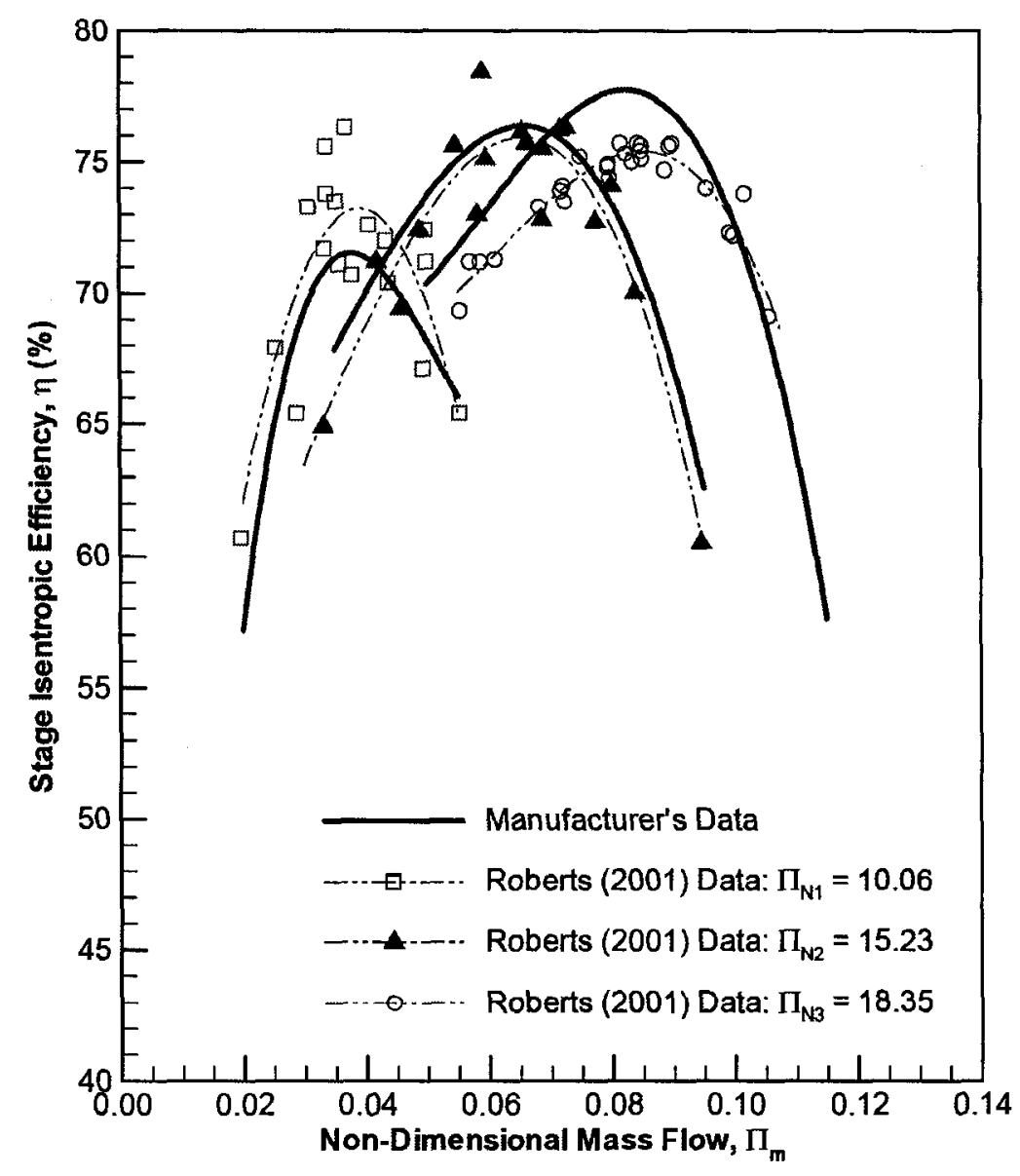

Figure 7.2: Efficiency characteristic of the T04E-50 compressor originally measured by Roberts (2001)

The total pressure ratio and efficiency characteristics obtained from the current test are shown in Figures 7.3 and 7.4. As seen, the differences between the measured and manufacturer's data are greater than those seen in Roberts (2001). This is attributed in part to wear on gaskets and seals during the disassembly and reassembly of the 
compressor which resulted in some leaks in the compressor casing. The disassembly and reassembly of the compressor may also have created slight changes in geometry since the work of Roberts (2001). These build differences may have resulted in small variations in the tip clearance which would affect the compressor performance. Additionally, the piping used to mate the compressor casing to the test stand had been damaged and needed to be repaired. These repairs resulted in larger steps in the flow passage than were originally present during the measurements by Roberts (2001). These steps are expected to have increased the losses.

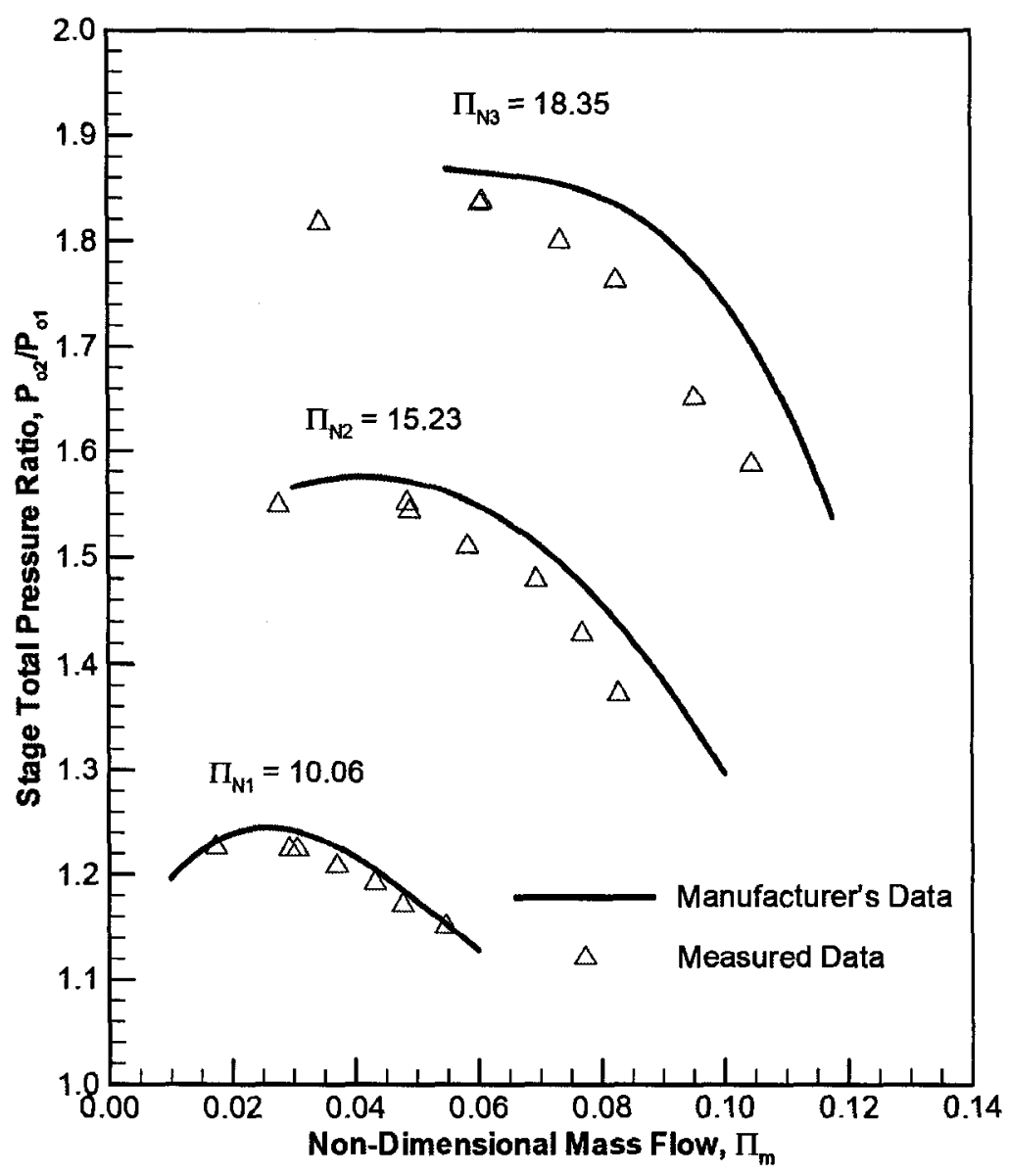

Figure 7.3: Measured total pressure ratio characteristic of the T04E-50 compressor compared to manufacturer's data 
During the experiments, it was only possible to set the rotational speeds within 500 RPM of the desired speed, from run to run. The variation of the desired speed results in variations of approximately $1 \%$ at the lowest shaft speed of 46,000 RPM. The performance parameters are therefore corrected for differences in non-dimensional speed parameter, as discussed in Section 4.6.3. The measured data were collected on multiple days and a single operating point on each constant speed line was repeated to verify the repeatability of the measurements.

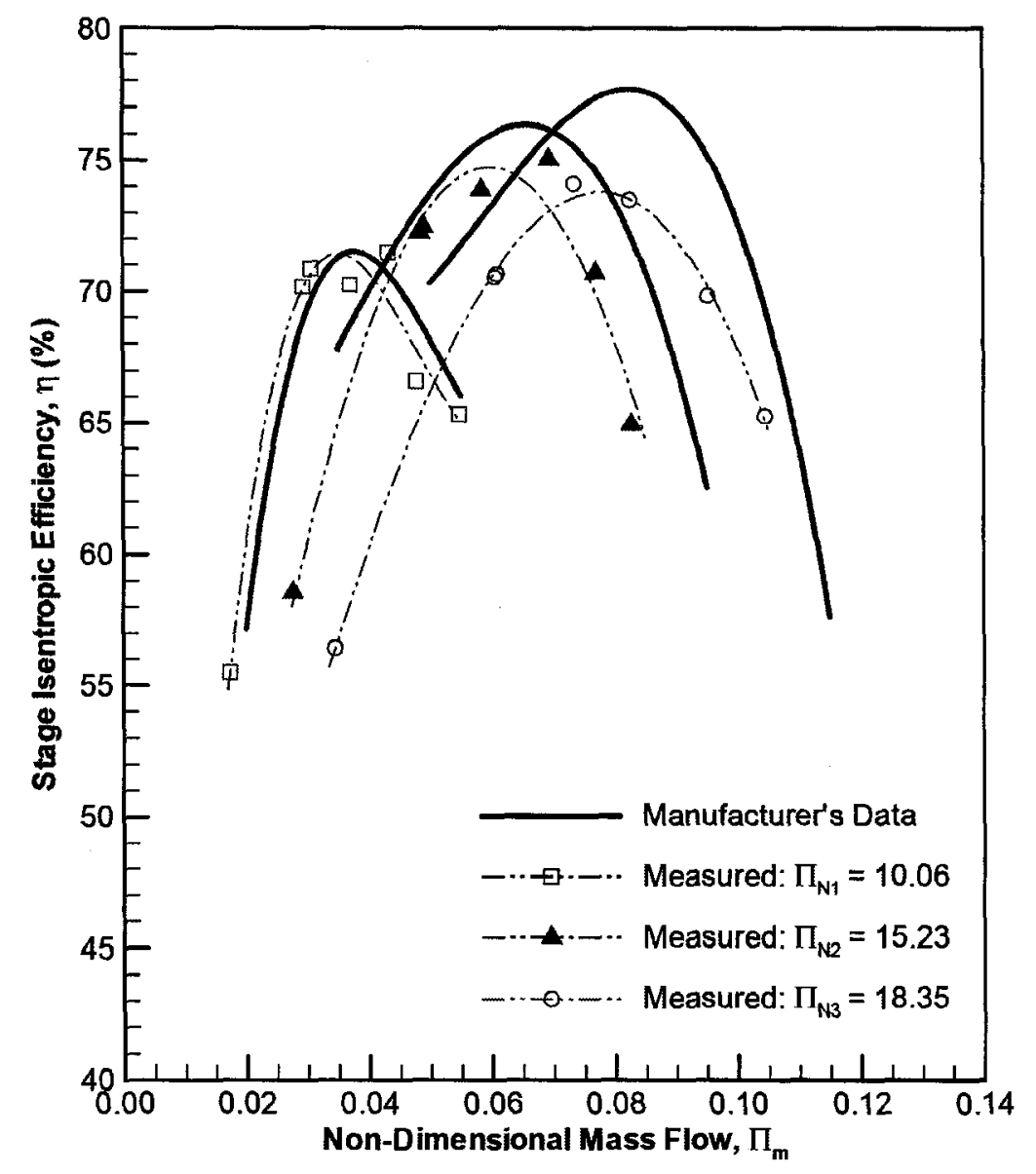

Figure 7.4: Measured efficiency characteristic of the T04E-50 compressor compared to manufacturer's data 


\subsection{EXPERIMENTAL PERformanCE Results}

\subsubsection{Effects of Rig Modifications on Stage Performance Characteristics}

The rotating test rig was originally only capable of providing overall stage performance data. The stage performance is obtained from measurements made upstream of the compressor inlet and in the discharge pipe of the test apparatus. It was desired to improve upon the capabilities of the test rig by adding pressure measurements to the compressor casing. The test rig modifications, described in Section 4.4, would give more detailed aerodynamic data for both the impeller and the volute. Included in the improvements was the installation of rotatable total pressure probes located within the vaneless diffuser, downstream of the impeller. It was hoped that these single-hole rotatable probes could function together, similarly to a multi-hole probe, to provide both total pressure and flow angle measurements near the impeller outlet. In total eight new probes of $3.2 \mathrm{~mm}$ diameter were inserted which resulted in blockage of approximately $10 \%$ of the flow passage area.

The new total pressure probes affect the measured overall stage performance since they are located upstream of the outlet measurement plane. Figure 7.5 and 7.6 show the stage performance characteristics with and without the new total pressure probes installed in the diffuser passage. The reference operating points indicated on Figure 7.5 correspond to the conditions for which the shroud surface and volute static pressure results were obtained. These results will be discussed later. Clearly the new probes have reduced the stage total pressure rise measured by the downstream total pressure probe. The decreased total pressure rise is due to the profile and mixing losses resulting from the new probes. As expected the pressure losses are larger at higher speeds. Also, the increased pressure losses have subsequently reduced the stage efficiency, as seen in Figure 7.6. 


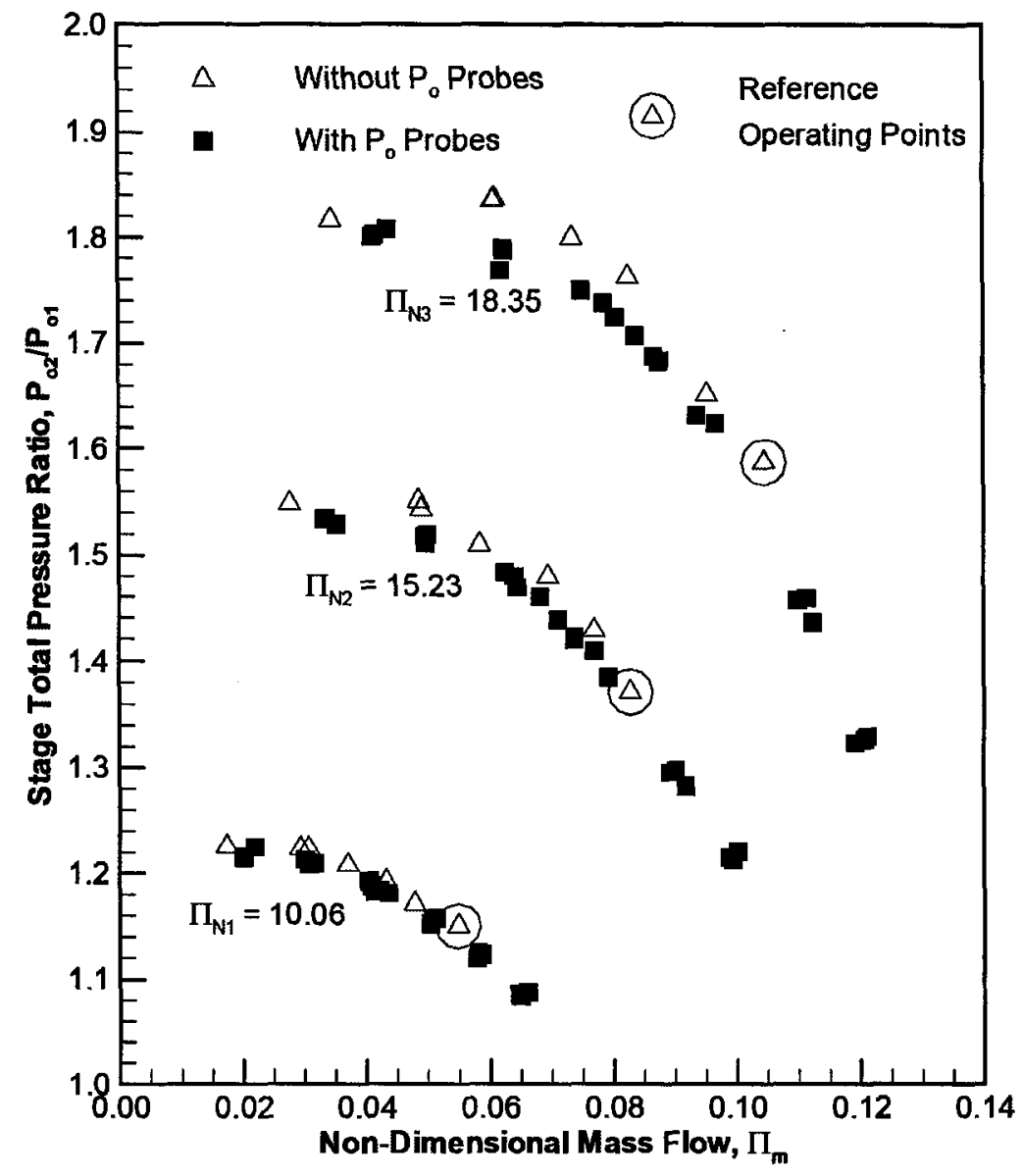

Figure 7.5: Stage total pressure ratio characteristic of the T04E-50 compressor with and without new total pressure probes

It seemed possible that the increased flow blockage from the new total pressure probes could affect the operating mass flow range of the compressor. However, the measured data are not sufficient to show any differences in the surging and choking mass flow rates since it does not span the complete mass flow range of the compressor. The complete range of the compressor cannot be measured because it is not possible to choke the compressor in the test rig due to significant total pressure losses within the circuit. 


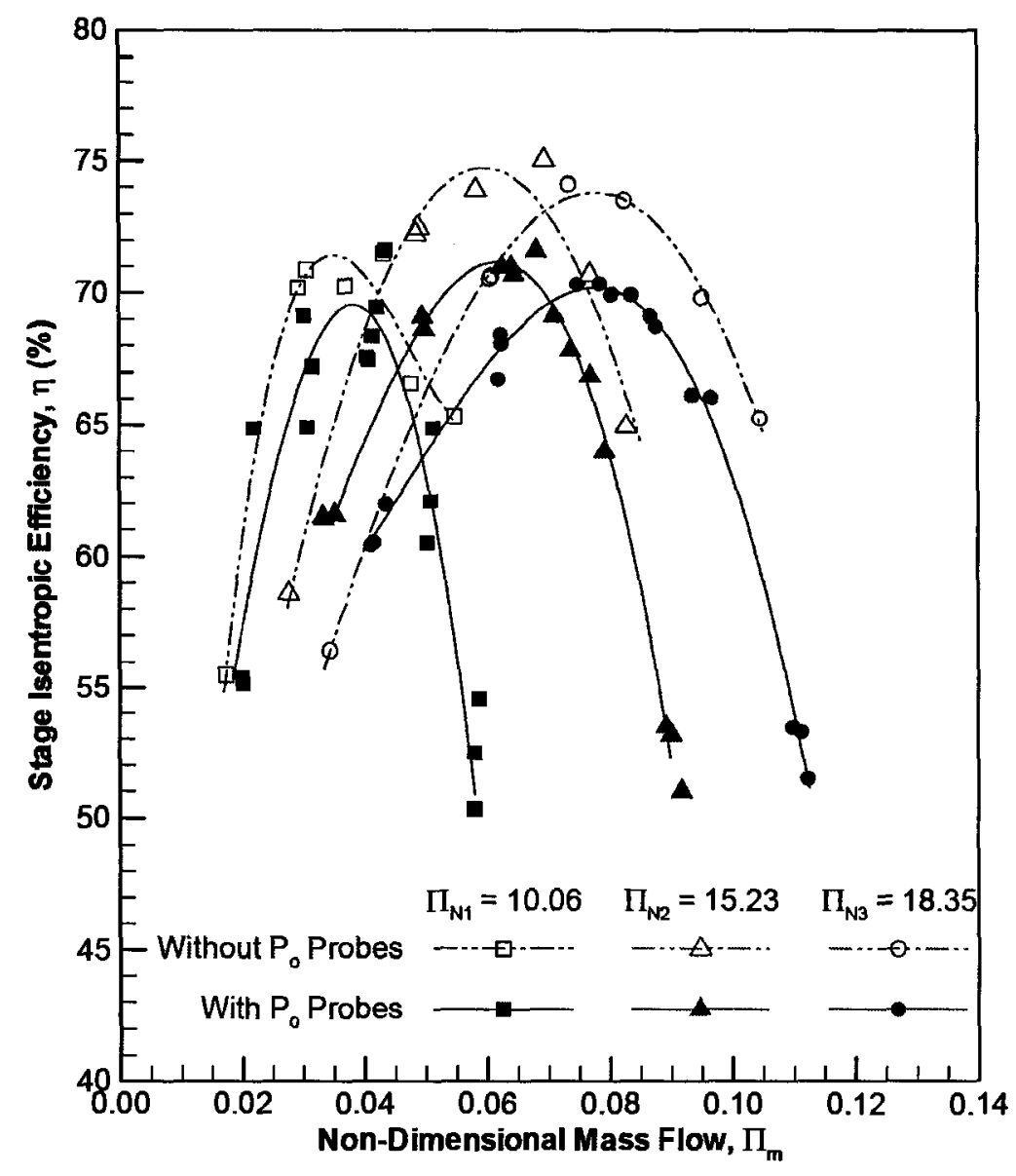

Figure 7.6: Effect of total pressure probes on the stage efficiency characteristic of the T04E-50 compressor

As noted, the compressor stage pressure ratio and isentropic efficiency have been reduced by the test rig modifications. Unfortunately, there is no manufacturer's data available for the impeller performance alone and therefore no means of determining the effect on the impeller performance. The new total pressure probes are located about 13 $\mathrm{mm}$ or 4 probe diameters downstream of the impeller. It is possible, although unlikely, that the presence of the probes is propagated upstream and is affecting the outlet flow from the impeller. However, there is no way of detecting such an effect. The new total pressure probes are located within the vaneless diffuser passage but the external geometry of the compressor casing did constrain their placement. 


\subsubsection{Impeller versus Stage Performance Characteristics}

The installation of the new total pressure probes provided the ability to measure the performance of the impeller independently of the compressor stage. The new total pressure probes were manufactured specifically for use in this study and their geometry is detailed in Section 4.4.1. The sensing hole of the manufactured probes is located on the cylindrical surface of the probe stem. The probes can then be rotated and the sensing hole can be aligned at multiple angles relative to the flow. In total, eight such single-hole total pressure probes were installed circumferentially around the impeller outlet. For a given operating point, all probes are fixed to a known angle relative to the radial axis of the impeller. The eight individual probe measurements are averaged yielding a circumferentially averaged pressure measurement for the specified angle of the probes. Measurements are then repeated for five probe angles for the same operating condition. A second order polynomial is fitted to the data and the peak of the curve is used to estimate the total pressure. The flow angle is then taken as the probe angle at which the curve is a maximum. A more detailed discussion of the data reduction is given in Section 4.6.

Figures 7.7 and 7.8 show the impeller pressure ratio and efficiency characteristics obtained using the new total pressure probe measurements. For comparison the overall stage performance data are also shown. As expected the total pressure rise and isentropic efficiency of the impeller are greater than for the complete stage at a given mass flow. The differences are the result of the pressure losses incurred in the downstream volute and piping. The measured outlet total temperature for both the impeller and stage efficiency was obtained from the same existing total temperature probe located in the discharge pipe of the test apparatus. Therefore, any increase in efficiency due to heat transfer is affecting both data sets equally and the difference in the impeller and stage efficiency estimates are a result of differing pressure losses alone. 


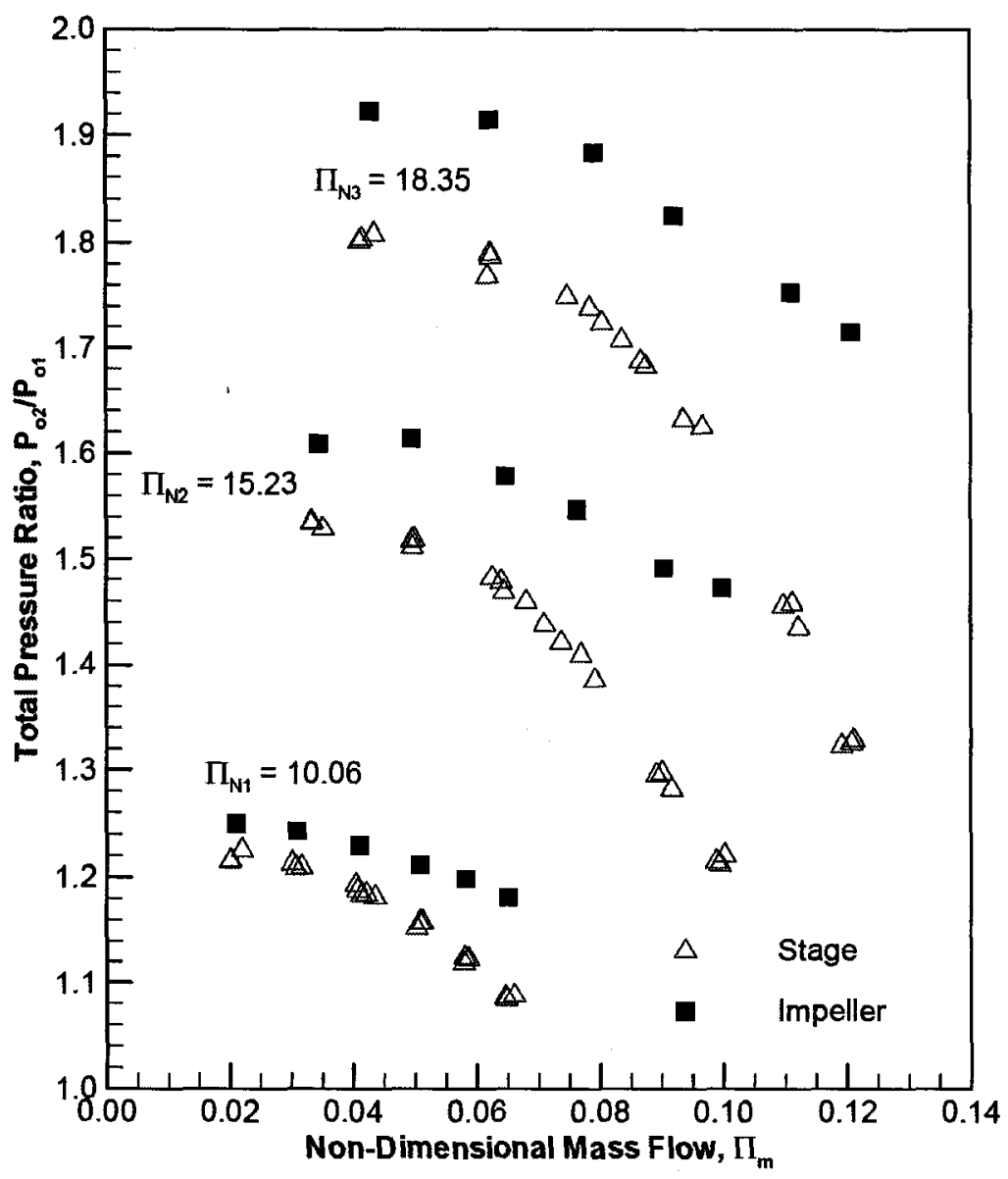

Figure 7.7: Measured impeller and stage total pressure ratio characteristic of the T04E-50 compressor 


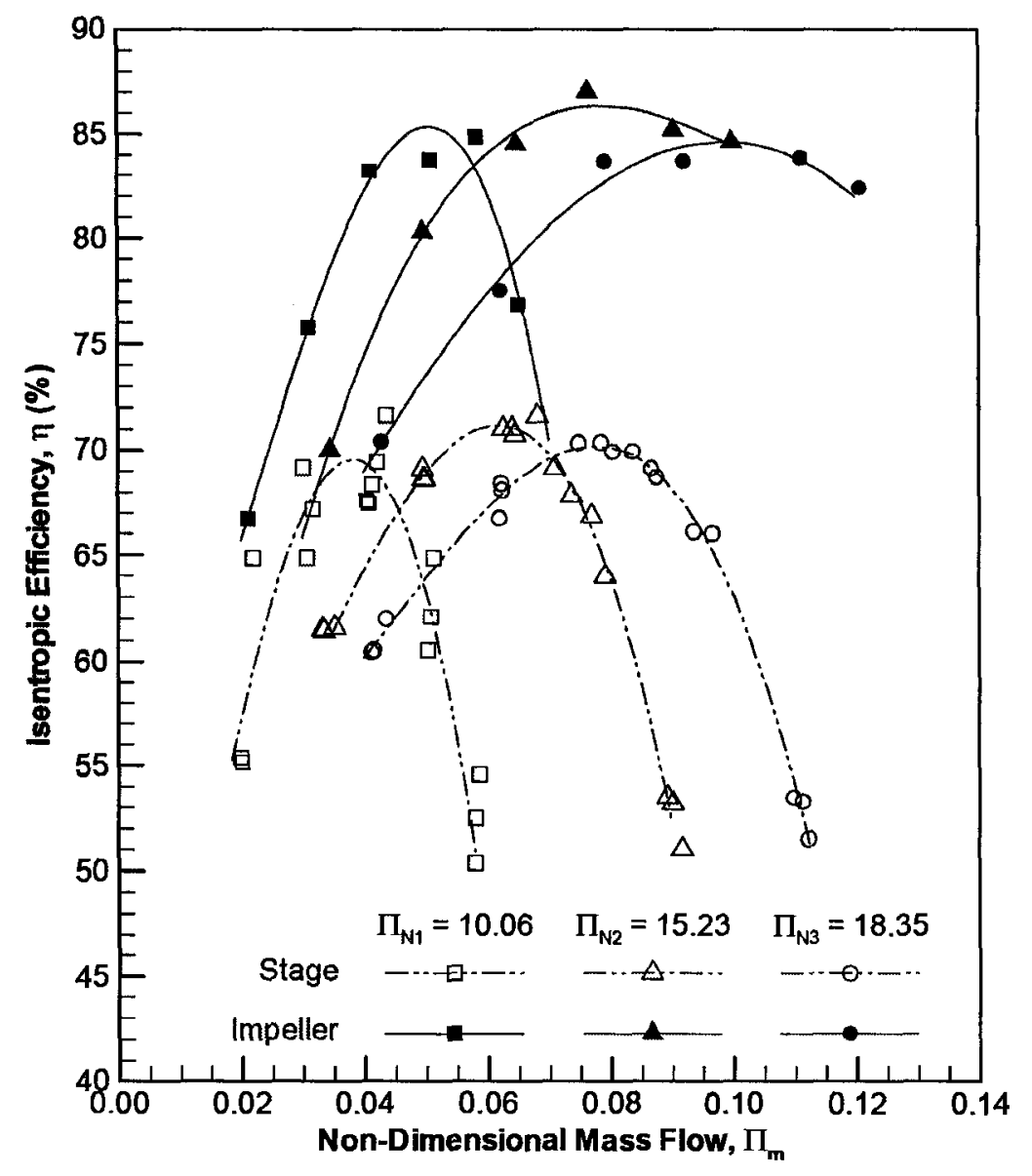

Figure 7.8: Measured impeller and stage efficiency characteristic of the T04E-

50 compressor

\subsubsection{Impeller Circumferential Variations Measured by Rotatable Total Pressure Probes}

The data reduction technique used in the previous section was used to estimate the total pressure and efficiency of the impeller at a given mass flow. However this technique masks any variations in flow around the circumference. Thus, an alternative data reduction method was used to estimate the circumferential variation of total pressure and flow angle around the impeller outlet. To determine the circumferential variations, the total pressure and flow angle at each total pressure probe must be estimated 
separately. To do this the total pressure and flow angle at each pressure probe was calculated from the five measurements from the given probe. A more detailed description of this methodology is given in Section 4.6.

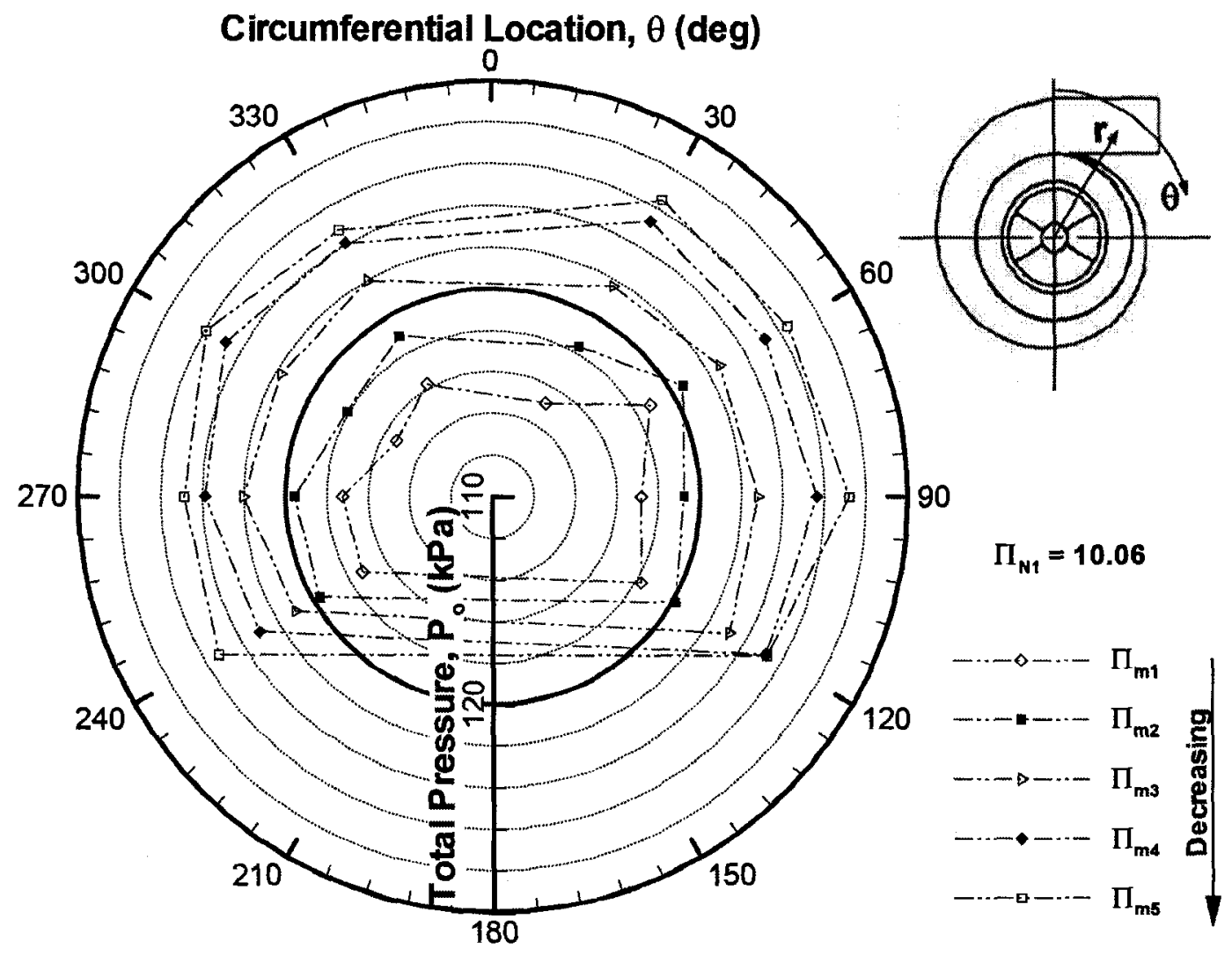

Figure 7.9: Circumferential variation of total pressure measured at the T04E-50 impeller outlet

Figure 7.9 shows the eight circumferential total pressure estimates for multiple mass flow rates, at a non-dimensional speed of 10.06. The straight lines used to connect the individual points are intended to 'guide the eye' and do not imply specific trends between the data points. This is especially true between the circumferential locations of $120^{\circ}$ to $240^{\circ}$. As can be seen, there is no obvious trend in the circumferential total pressure variation. The circumferential change in total pressure along a given non- 
dimensional mass flow is approximately $2 \mathrm{kPa}$ or, less than $2 \%$ of the minimum measured total pressure.

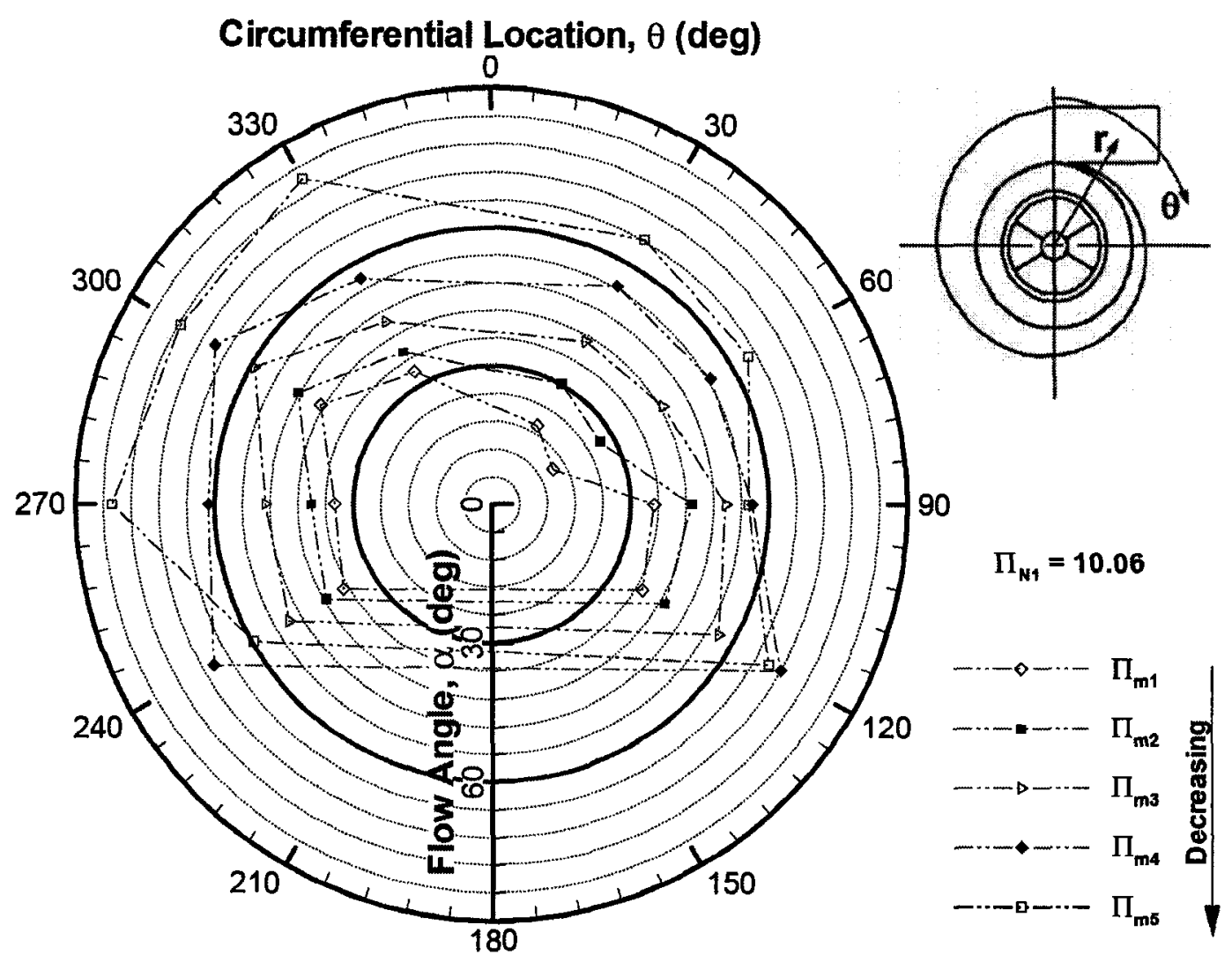

Figure 7.10: Circumferential variation of flow angle measured at the T04E-50 impeller outlet

In addition to the total pressure estimates provided by the new pressure probes, the flow angle can also be estimated. Figure 7.10 shows the circumferential variation of flow angle for the same operating points as seen in Figure 7.9. Again the lines connecting a given non-dimensional mass flow are only intended to 'guide-the-eye'. Regardless, it is evident that there is a region of lower flow angle at the $30^{\circ}$ and $60^{\circ}$ circumferential locations. This corresponds to the region of the tongue and could indicate its influence on the impeller flow. On the other hand, there seemed to be little effect on the total pressure, as seen from Figure 7.9. 
The difference in the circumferential variation of total pressure and flow angle may in part be due to spanwise variations at the measurement plane. The flow angle gradient across the span predicted by ANSYS CFX is much larger than the similar total pressure gradient, as discussed in Section 7.4.3. The estimate of flow angle is therefore more sensitive to the spanwise position of the probe than the similar total pressure measurement.

\subsubsection{Static Pressure Variation on Shroud Surface}

The static pressure variation along the shroud surface was also measured with the modified test rig. Two radial rows of static pressure taps were drilled through the compressor casing at two circumferential locations as shown in Figure 7.11. Due to the constraints of the external geometry of the compressor casing the radial spacing of the taps was different for the two rows and only four of the radial locations were matched.

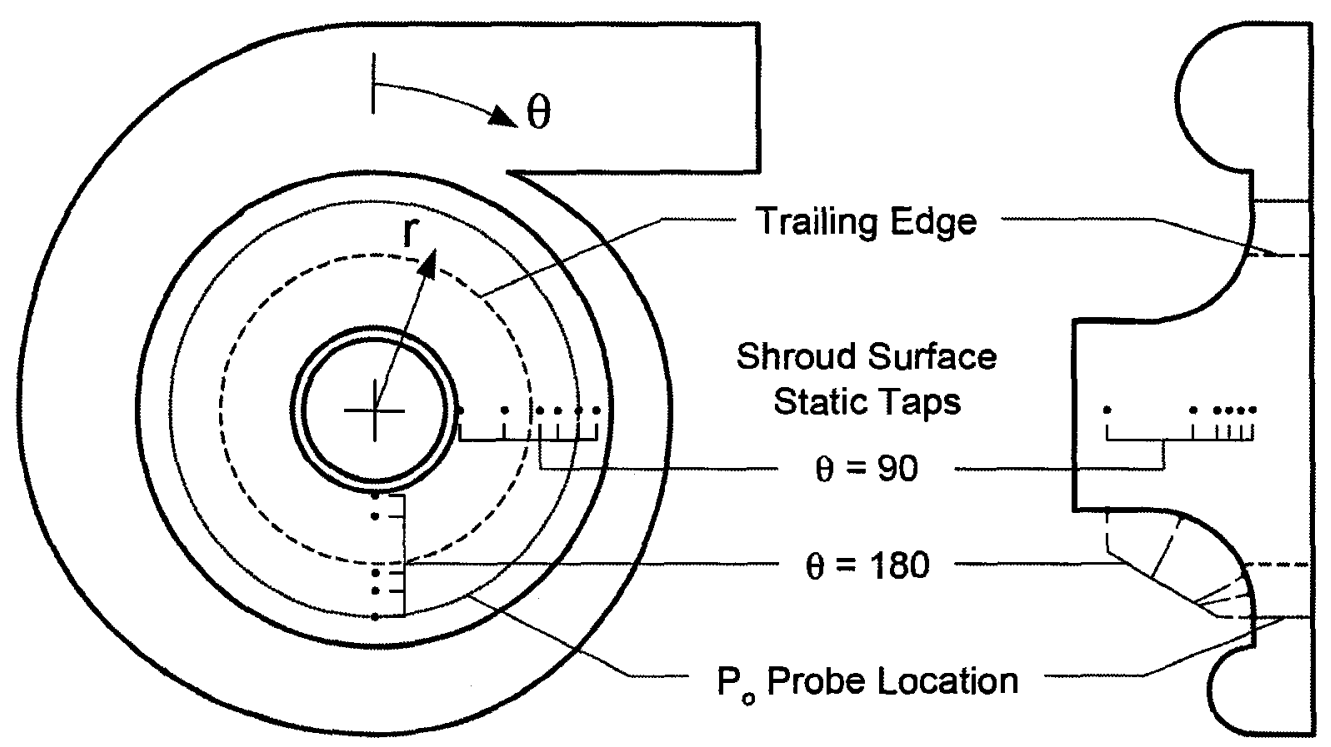

Figure 7.11: Schematic of the shroud surface static tap locations 


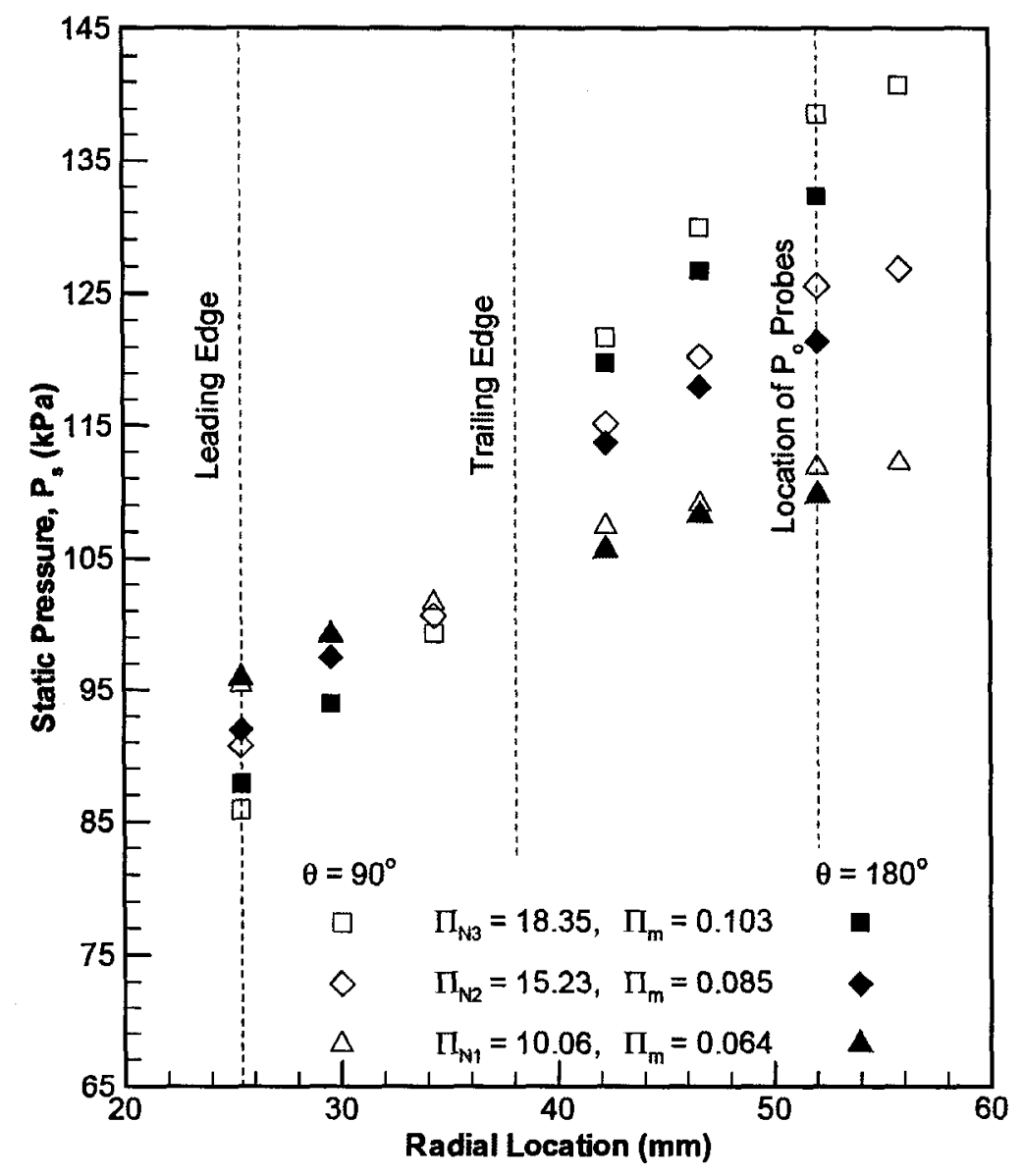

Figure 7.12: Static pressure variation measured on the shroud surface of the T04E-50 compressor

Figure 7.12 shows the static pressure variation on the shroud for the three nondimensional speeds for the row of taps at the $90^{\circ}$ and $180^{\circ}$ position. The reference operating points used are indicated on Figure 7.5. The data are shown in dimensional form and illustrate the increased pressures and gradients present at the higher rotational speeds. The same data are non-dimensionalized and compared with the CFD predictions in Section 7.4.4.

As expected the static pressure increases with radius and the gradient increases with speed. The figure also shows that the static pressures are higher at the $90^{\circ}$ 
circumferential location downstream of the trailing edge, particularly at the highest speed parameter. Since the $90^{\circ}$ position is close to the tongue region, it is more evidence of the tongues influence on the flow. However, the static pressures are similar within the impeller and seemingly the influence of the tongue is limited to the diffuser passage.

\subsubsection{Volute Static Pressure Variation}

Eight static pressure taps were also added to the volute of the T04E-50 compressor. Their locations are shown schematically in Figure 7.13.

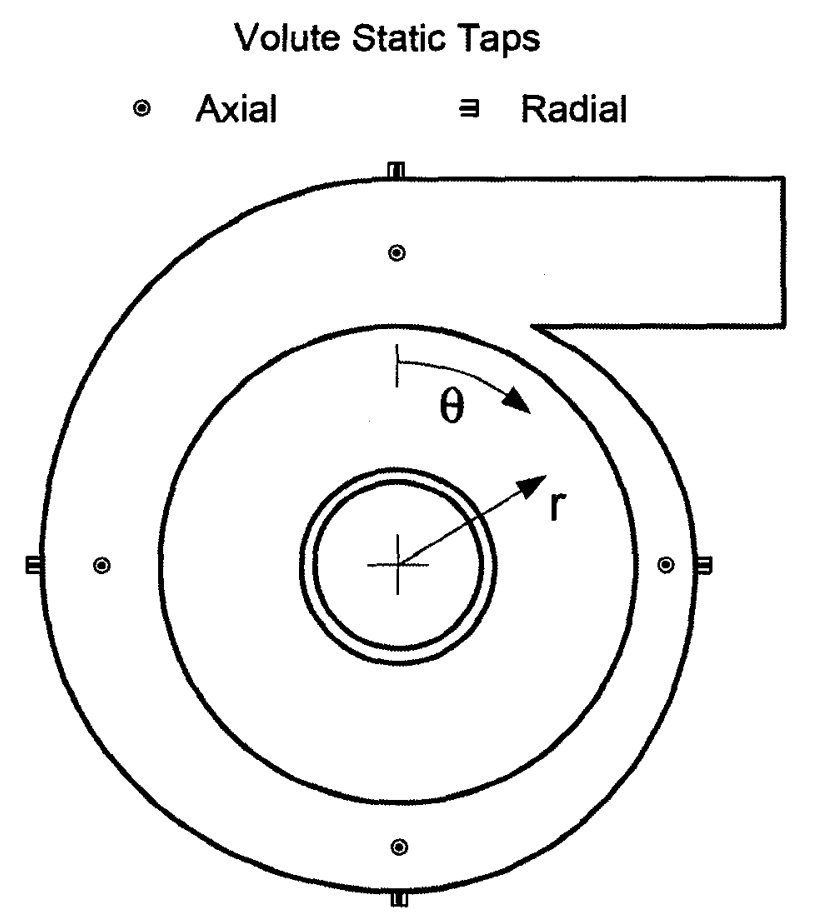

Figure 7.13: Schematic of the volute static tap locations

Figure 7.14 shows the circumferential static pressure variation around the volute measured by both the axial and radial aligned taps. The reference operating points used are indicated on Figure 7.5. As seen in Figure 7.14, the pressures measured by the radial and axial oriented taps are very similar and indicate a maximum static pressure between the $90^{\circ}$ and $180^{\circ}$ circumference locations for all non-dimensional speeds. 


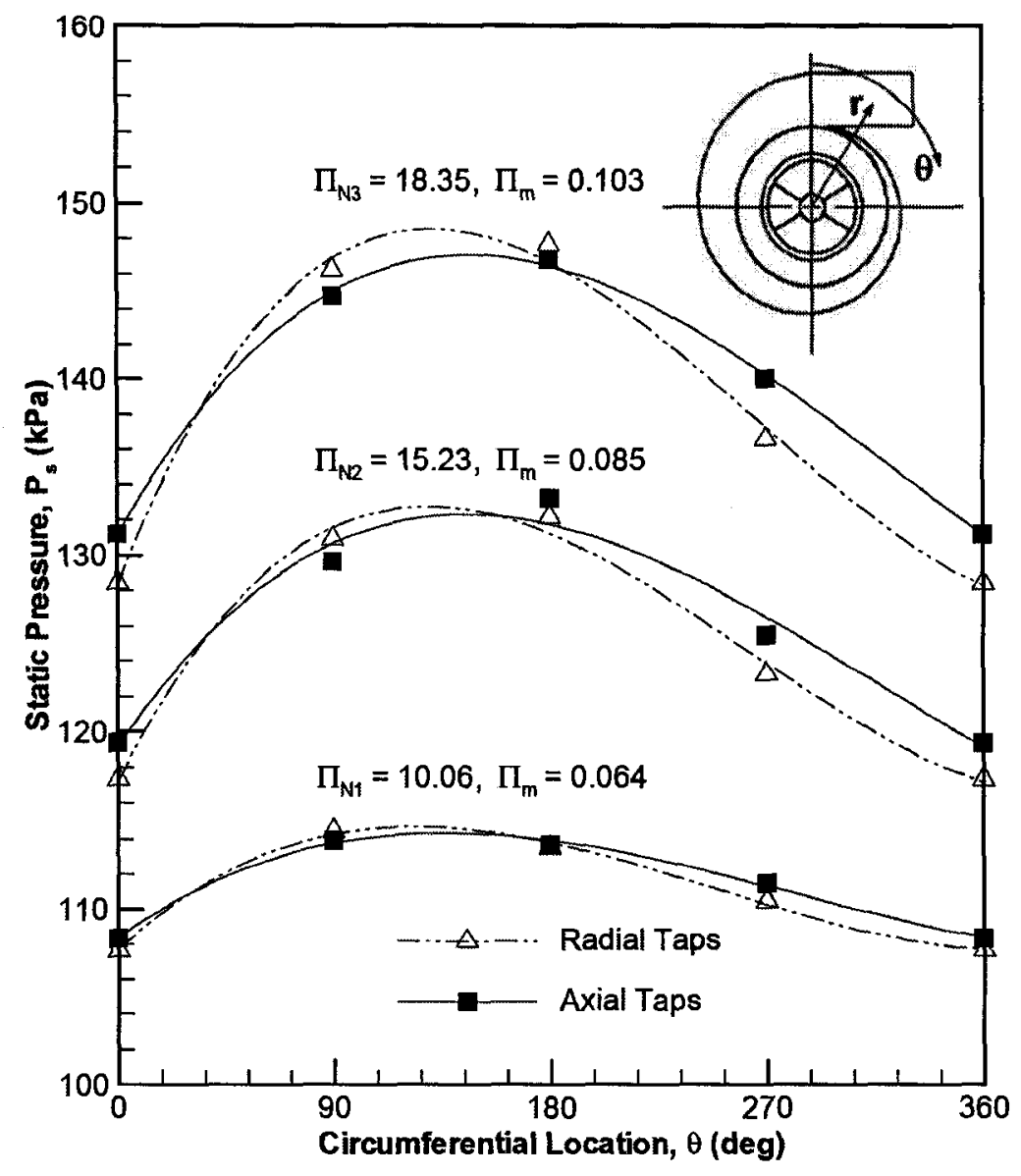

Figure 7.14: Circumferenctial variation of static pressure variation measured on the volute of the T04E-50 compressor

The maximum static pressures measured around the volute are approximately 147,130 and $115 \mathrm{kPa}$ for non-dimensional speeds of $18.35,15.23$ and 10.06 respectively. The maximum static pressures measured on the shroud surface are approximately 140 , 125 and $107 \mathrm{kPa}$ for the same non-dimensional speeds. Thus there is a small degree of pressure recovery between the last row of shroud surface taps and the volute. 


\subsection{TO4E-50 ImPELLER PERformanCE PREDICTIONS USing ANSYS CFX}

\subsubsection{Impeller Performance Characteristics}

CFD predictions of the Turbonetics T04E-50 impeller were performed using ANSYS CFX. Because a solids-model of the volute did not exist the performance predictions were limited to just the impeller. The rotatable total pressure probes added during the test rig modifications allowed the impeller performance to be measured and compared to the corresponding CFD predictions. Unfortunately manufacturer's data were not available for comparison with the impeller-only-performance measurements made with the new total pressure probes.

The performance of the T04E-50 impeller was predicted and expressed in terms of the same non-dimensional performance parameters as seen previously in Section 7.3.2. Figures 7.15 and 7.16 compare the predicted total pressure ratio and efficiency characteristics of the impeller to the measured performance data. The reference operating points shown were used to examine the more detailed aerodynamic performance in Sections 7.4.3 and 7.4.4. For both figures the outlet values of total pressure from the CFD predictions are obtained by mass-averaging along a thin ring that is $1 \mathrm{~mm}$ high at the same radius as the measurements. The ring height for averaging corresponds to the diameter of the hole in the total pressure probes. All other inlet and outlet flow quantities obtained from the predictions are mass-averaged across the entire passage area at the same radius. 


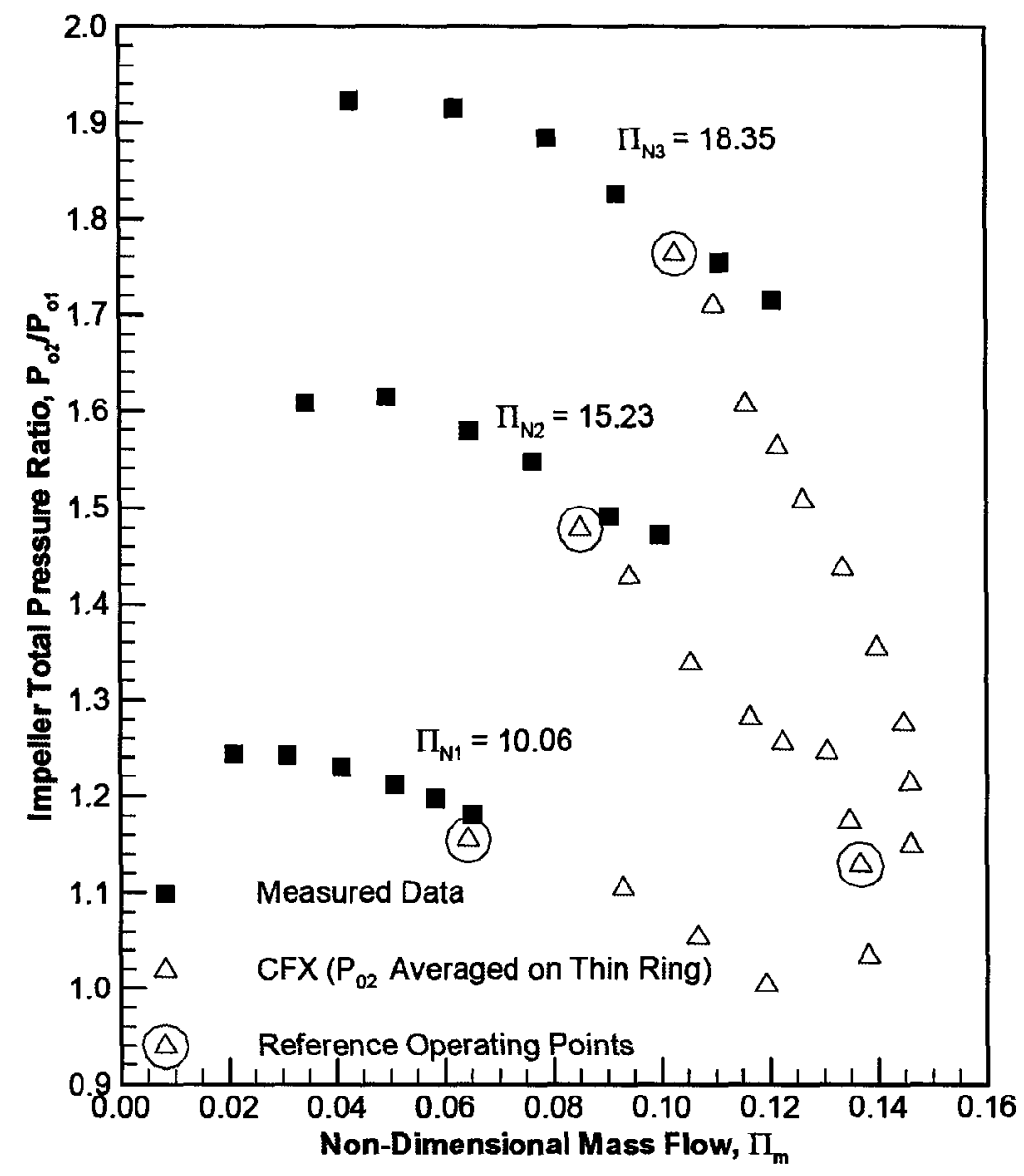

Figure 7.15: Total pressure ratio predicted by ANSYS CFX using outlet total pressure values mass-averaged over a thin ring, compared to measured data for the T04E-50 impeller

As seen in Figures 7.15 and 7.16 the measured and predicted performance characteristics for a given constant speed overlap for a small range of non-dimensional mass flows. Because of high losses in the test circuit it was not possible to obtain performance data for operating points at higher mass flow rates than those shown. Additionally, difficulties were encountered in trying to obtain converged solutions with CFX for lower mass flow than those shown. This is believed to be a result of difficulties for the steady-state solver to predict the onset of blade stall. In retrospect it might have been useful to obtain time accurate solutions. 


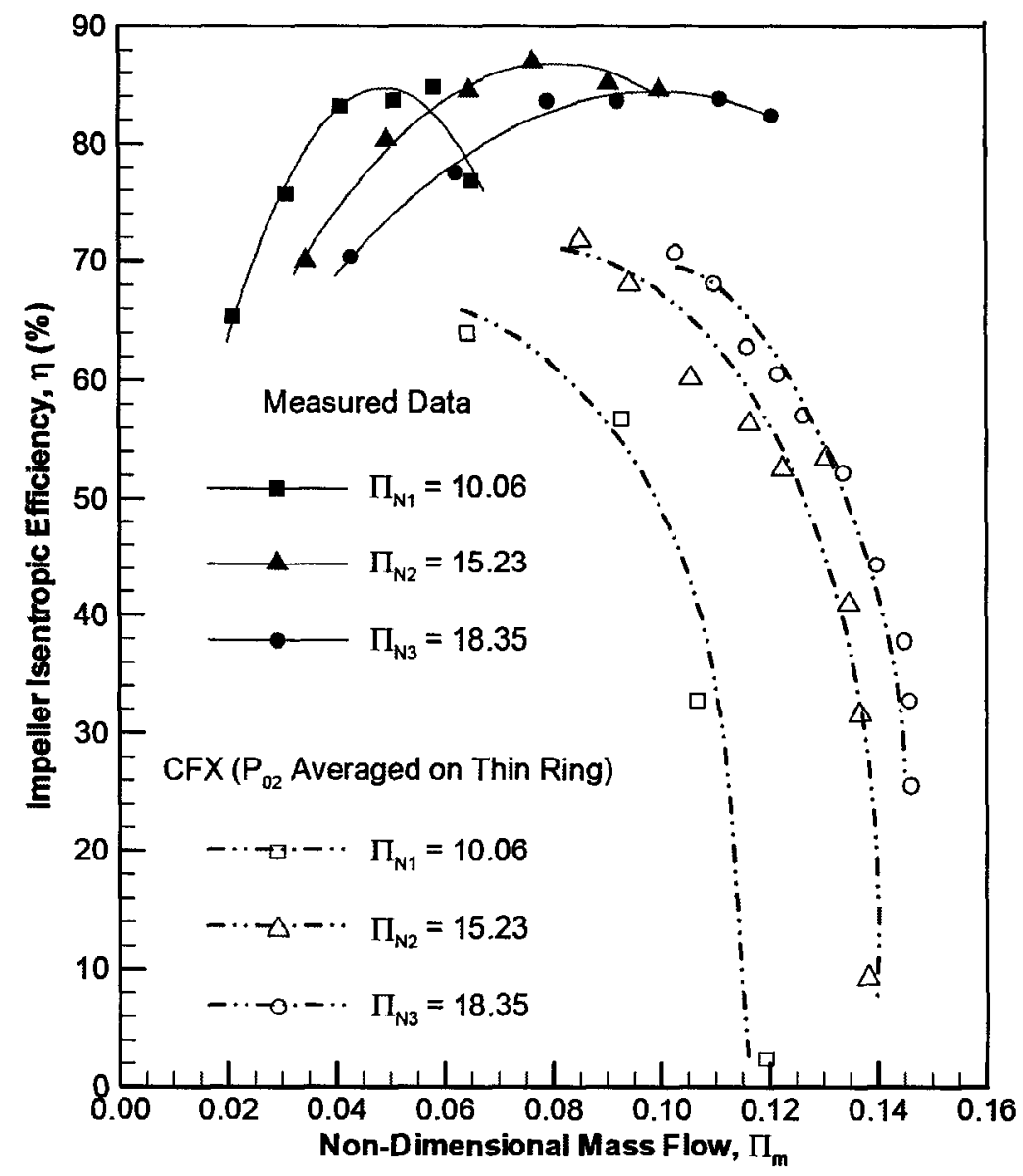

Figure 7.16: Efficiency predicted by ANSYS CFX using outlet total pressure values mass-averaged over a thin ring, compared to measured data for the T04E-50 impeller

For mass flow rates that overlap the measured and predicted pressure ratios are similar as seen in Figure 7.15. However, the predicted constant speed operating lines are steeper than for the measured data, particularly at higher rotational speeds. From Figure 7.16 it is evident that CFX is predicting a much lower isentropic efficiency than measured. The differences seen in efficiency may be a result of differences in the tip leakage between the computations and the experiments. The solids model of the impeller used for the computations was produced from a three-dimensional scan of the actual T04E-50 impeller. However, the compressor casing could not be scanned. Instead, the 
tip clearances were estimated with geometric measurements of the impeller and casing and therefore have a high level of uncertainty. The differences in performance characteristics may therefore be due in part to differences in the modelled and actual tip clearances.

It is also seen from Figures 7.15 and 7.16 that the constant speed lines are not as smooth as one would expect from CFD predictions. This is most noticeable in the efficiency predictions, which show some scatter around the fitted curve. This is believed to be a result of the thin ring used to mass-average the outlet total pressure. The ring height corresponds to approximately $3 \%$ of the blade passage height at the total pressure probe location. As discussed in Section 7.4.3 the distribution of total pressure at this location changes with mass flow rate. Thus the predicted total pressure will be affected by the relative position of the ring area to regions of high and low pressure. In an attempt to reduce the scatter seen in the CFD predictions, the outlet total pressure was also massaveraged across the complete flow area at the same radial location as the thin ring.

The overall performance characteristics predicted from the mass-averaged total pressure over the full flow area is compared to the measured performance data in Figures 7.17 and 7.18. Figure 7.17 shows that the pressure rise is being over-estimated by CFX while Figure 7.18 shows a lower predicted efficiency for a given operating point when compared to the measured performance. This is due to the outlet temperature measurements used to determine the measured efficiency. As stated, the impeller outlet total pressure was measured by the new total pressure probes within the diffuser passage. However, the outlet total temperature was measured downstream of the compressor. Despite best efforts to prevent heat loss from the test circuit it is expected that the total temperature decreases downstream. This results in an over estimate of the efficiency. 


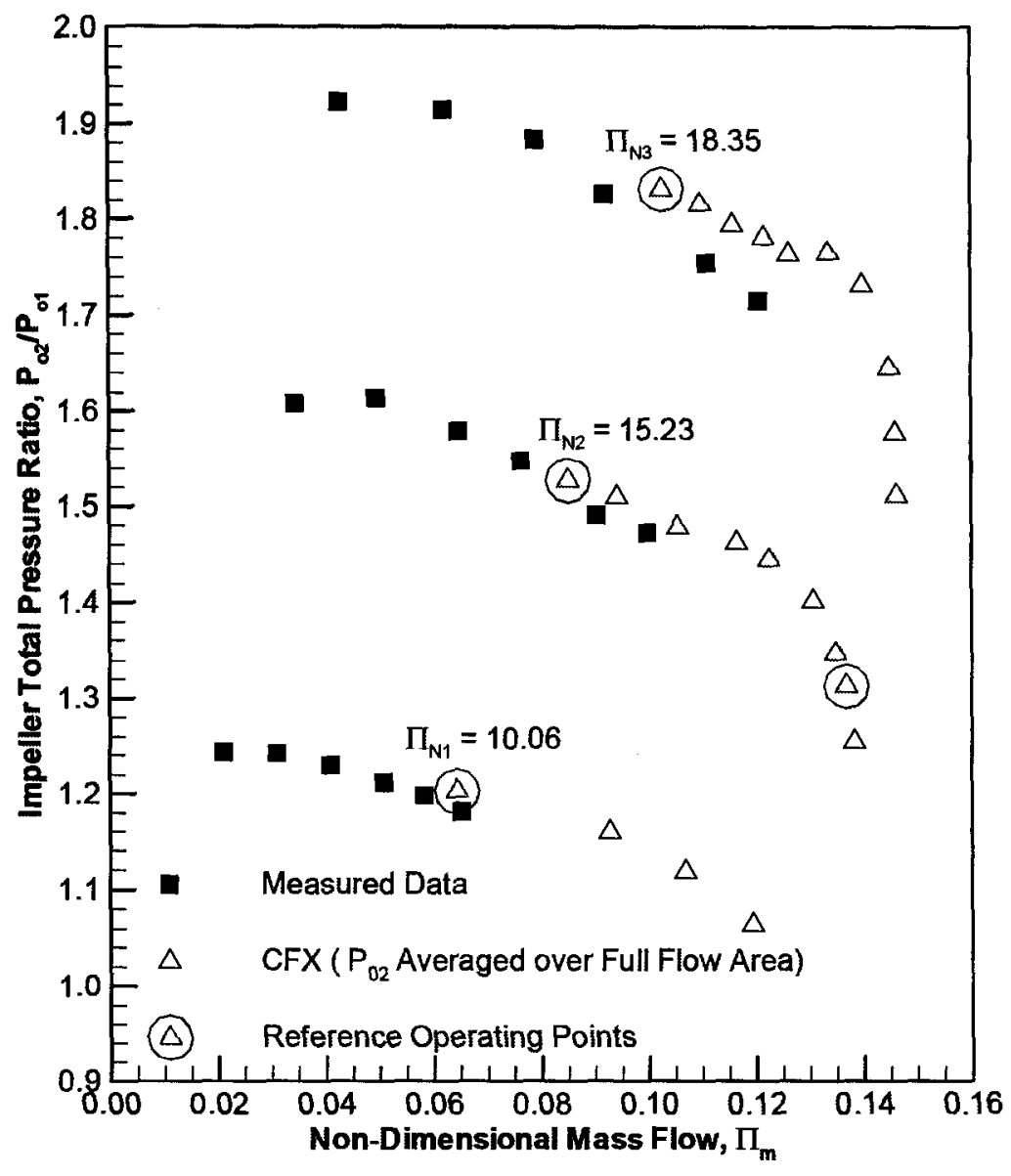

Figure 7.17: Total pressure ratio predicted by ANSYS CFX using outlet total pressure values mass-averaged across the full flow area, compared to measured data for the T04E-50 impeller

It is also evident from Figures 7.17 and 7.18 that the trends in pressure rise and efficiency predicted by CFX are closer to those seen in the measured data when the predictions are averaged over the full flow area. In addition, the predicted constant speed lines using the mass-averaged total pressure across the full flow area are flatter than those predicting using an average across the thin ring. As seen in Figure 7.17, the predicted constant speed lines are again not as smooth as one would expect and the reason is not clearly understood. With the exception of the operating points of maximum mass flow at non-dimensional speeds of 10.06 and 15.23 all other solutions presented in Figures 7.15 
to 7.18 were converged to maximum residuals of $10^{-5}$ and thus the trends in the predictions are not believed to be due to incomplete convergence.

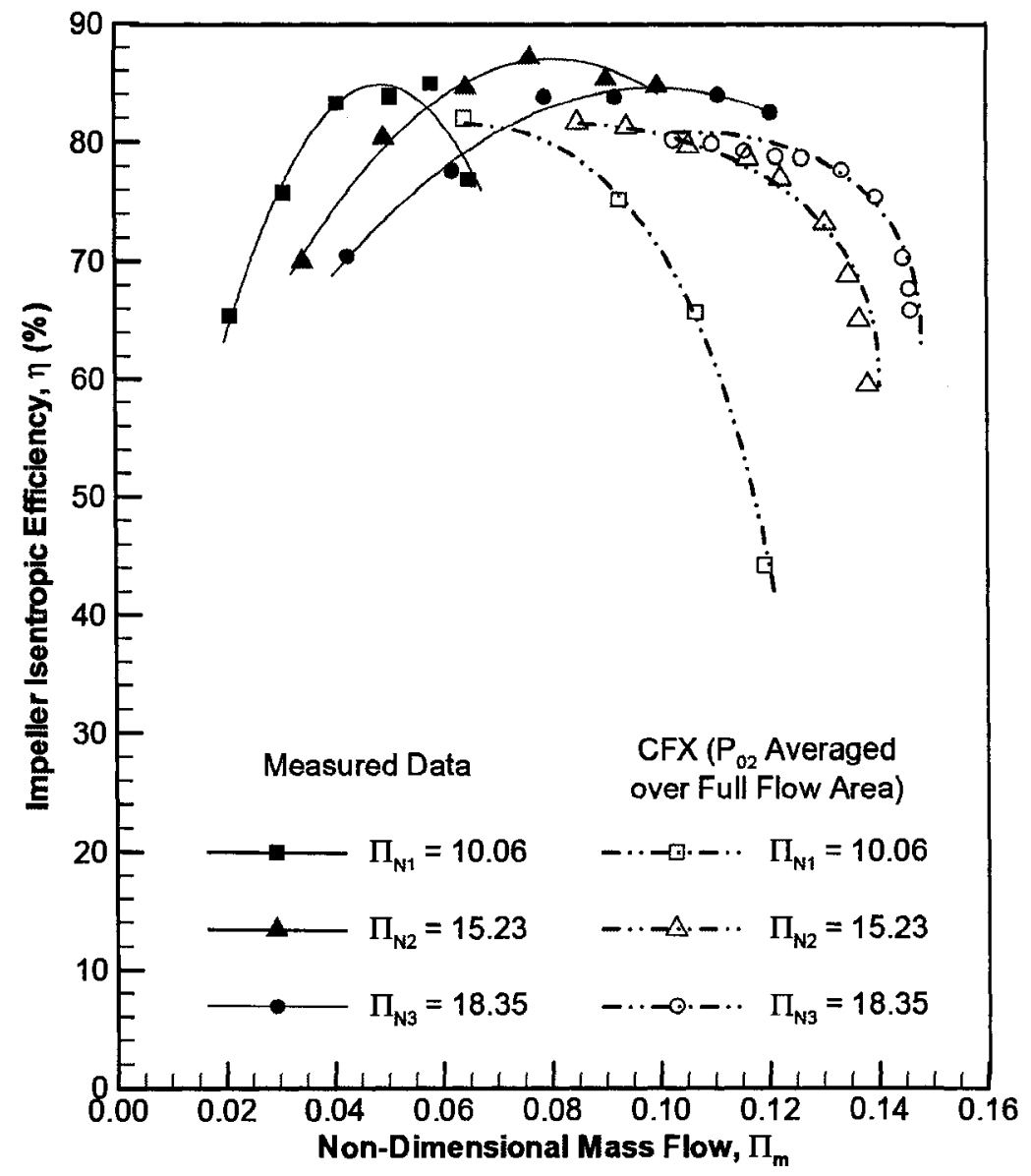

Figure 7.18: Efficiency predicted by ANSYS CFX using outlet total pressure values mass-averaged across the full flow area, compared to measured data for the T04E-50 impeller

\subsubsection{Exit Flow Angle Predictions for Varying Mass Flow Rates}

The flow angle data collected from the experiments was also compared to the CFD predictions. Because the computational case consisted of the rotor alone no circumferential variations are present except those from the pressure side to the suction side of the vanes. The assumption of periodicity around the circumference is used to 
simplify the computational case. In order to compare the predicted flow angles to those measured the mass-averaged flow angles were calculated for the same two annular rings as the total pressure and efficiency data presented in the previous section. Figures 7.19 and 7.20 show the exit flow angle for varying mass flow rates averaged over each of these two areas. Similar to the total pressure rise and efficiency the CFX predictions of the mass-averaged flow angle across the entire passage area (Fig. 7.20) is in closer agreement with the measured data than the mass-averaged flow angle over the thinner ring corresponding to the mouth of the probes (Fig. 7.19).

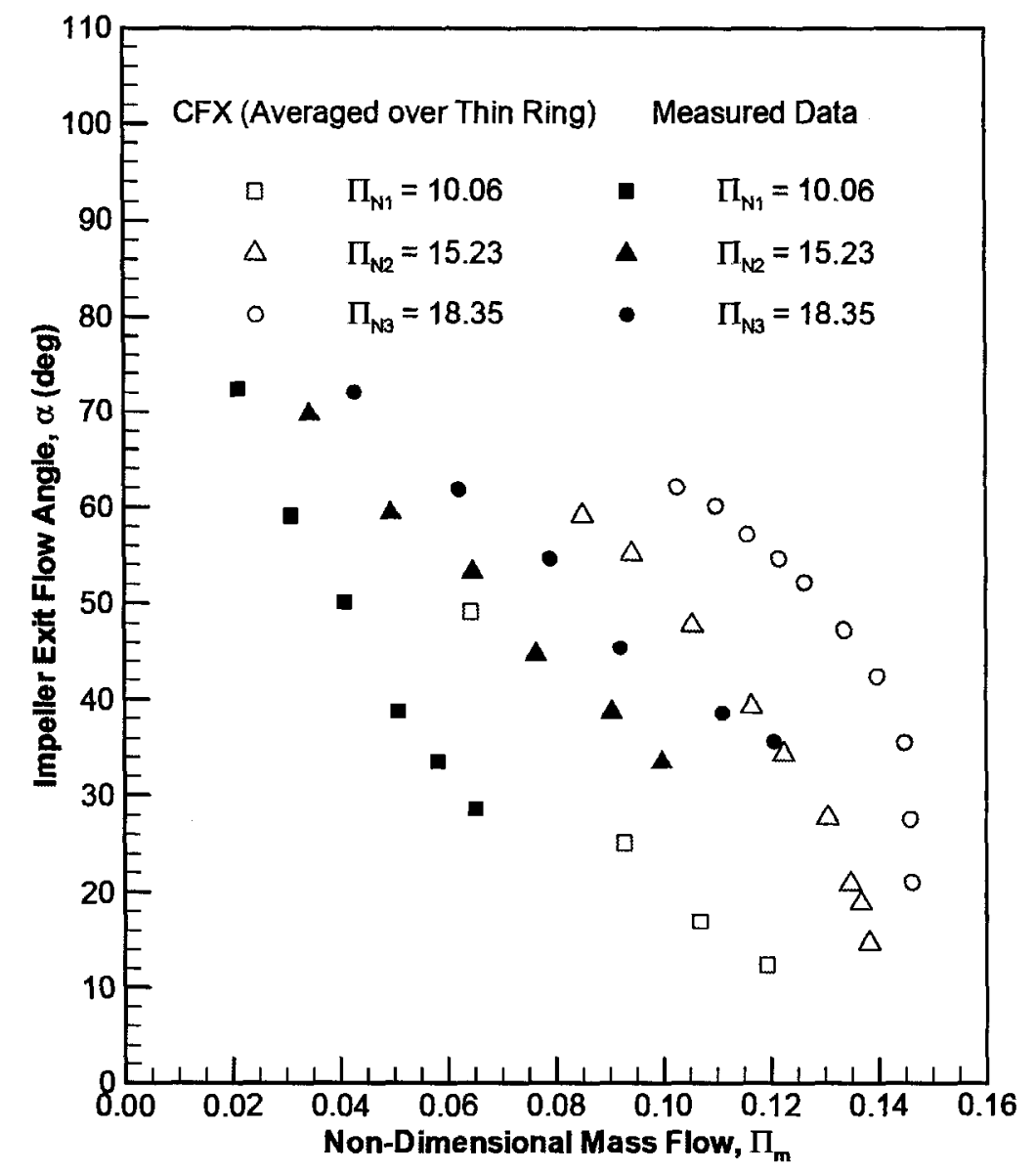

Figure 7.19: Exit flow angle predicted using ANSYS CFX mass-averaged across the thin ring area, compared to measured data for the T04E-50 impeller 
Figure 7.19 shows that the flow angle is over predicted when the thin ring is used for averaging. Figure 7.15 showed an under prediction of the corresponding massaveraged total pressure rise. As mentioned previously the flow angle and pressure rise are related. Thus the total pressure rise and exit flow angle should be similarly under or over predicted. As will be discussed in Section 7.4.3 there is evidence of two fluid cores present in the total pressure distribution, particularly at increased mass flow rates. The flow angle distribution, also seen in Section 7.4.3, is noticeably different from the total pressure distribution, with no evidence of the fluid cores and only spanwise variations. When averaging over the complete flow area the predicted trends in the pressure rise and flow angle variations are similar, as seen by comparison of Figures 7.16 and 7.20. It is therefore possible that the total pressure and flow angle are not similarly under or over predicted when averaging over the thin ring because of the differences in the total pressure and flow angle distribution for a given mass flow.

The passage area mass-averaged outlet values provide closer agreement between all of the predicted and measured performance characteristics than the probe area massaveraged values. However, this agreement is not expected to hold if the measurement and computational techniques were applied to a different compressor design because the experimental measurements do not represent an accurate average of the complete flow area. 


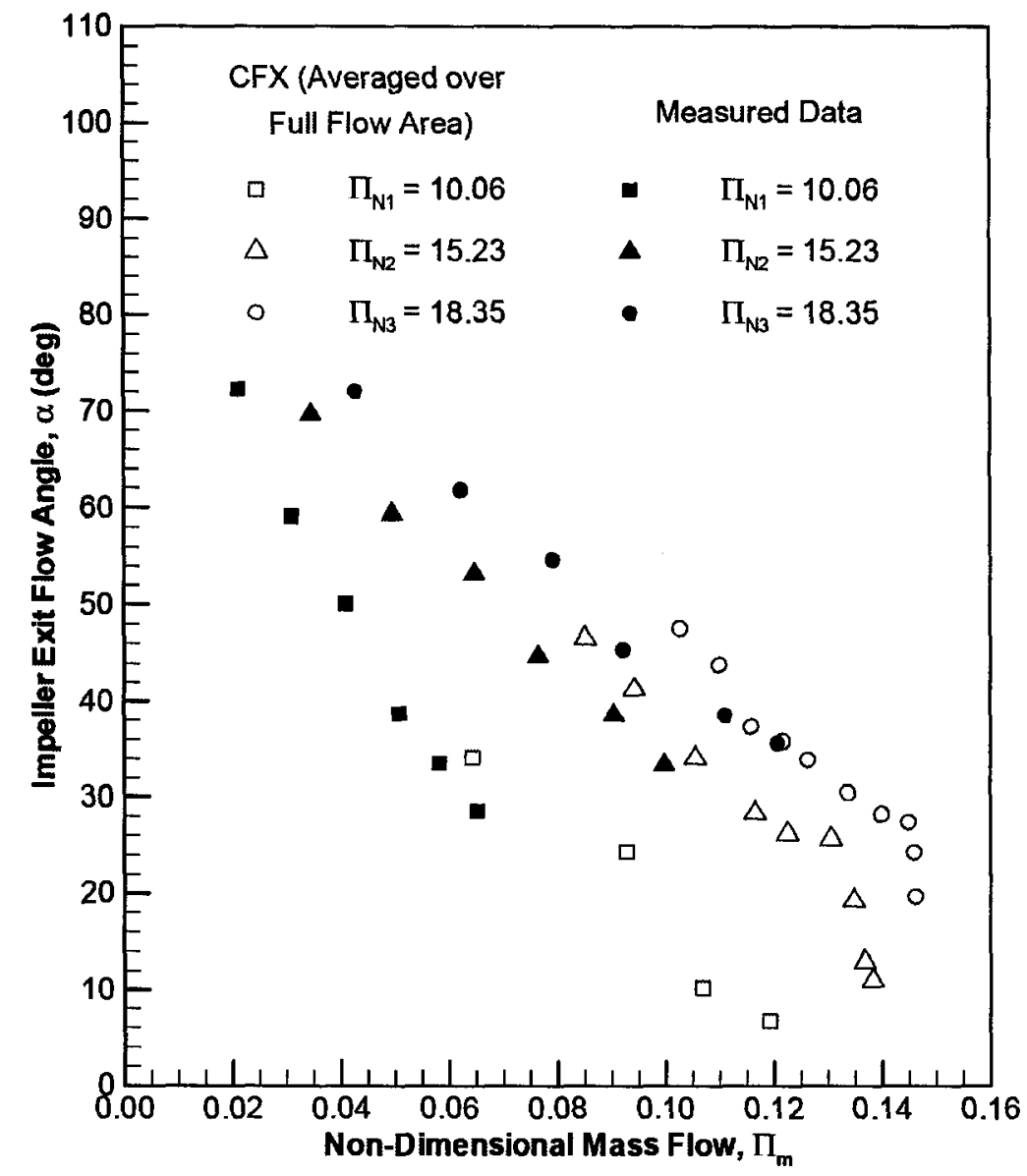

Figure 7.20: Exit flow angle predicted using ANSYS CFX mass-averaged across the full flow area, compared to measured data for the T04E-50 impeller

\subsubsection{Downstream Total Pressure and Flow Angle Distributions}

ANSYS CFX can provide more detailed aerodynamic information than the experiments. The CFX predictions can therefore be used to help interpret the experimental measurements.

The contours of predicted total pressure and flow angle downstream of the impeller are shown in Figures 7.21 and 7.22 respectively. The reference operating points used are indicated on Figures 7.15 and 7.17. 

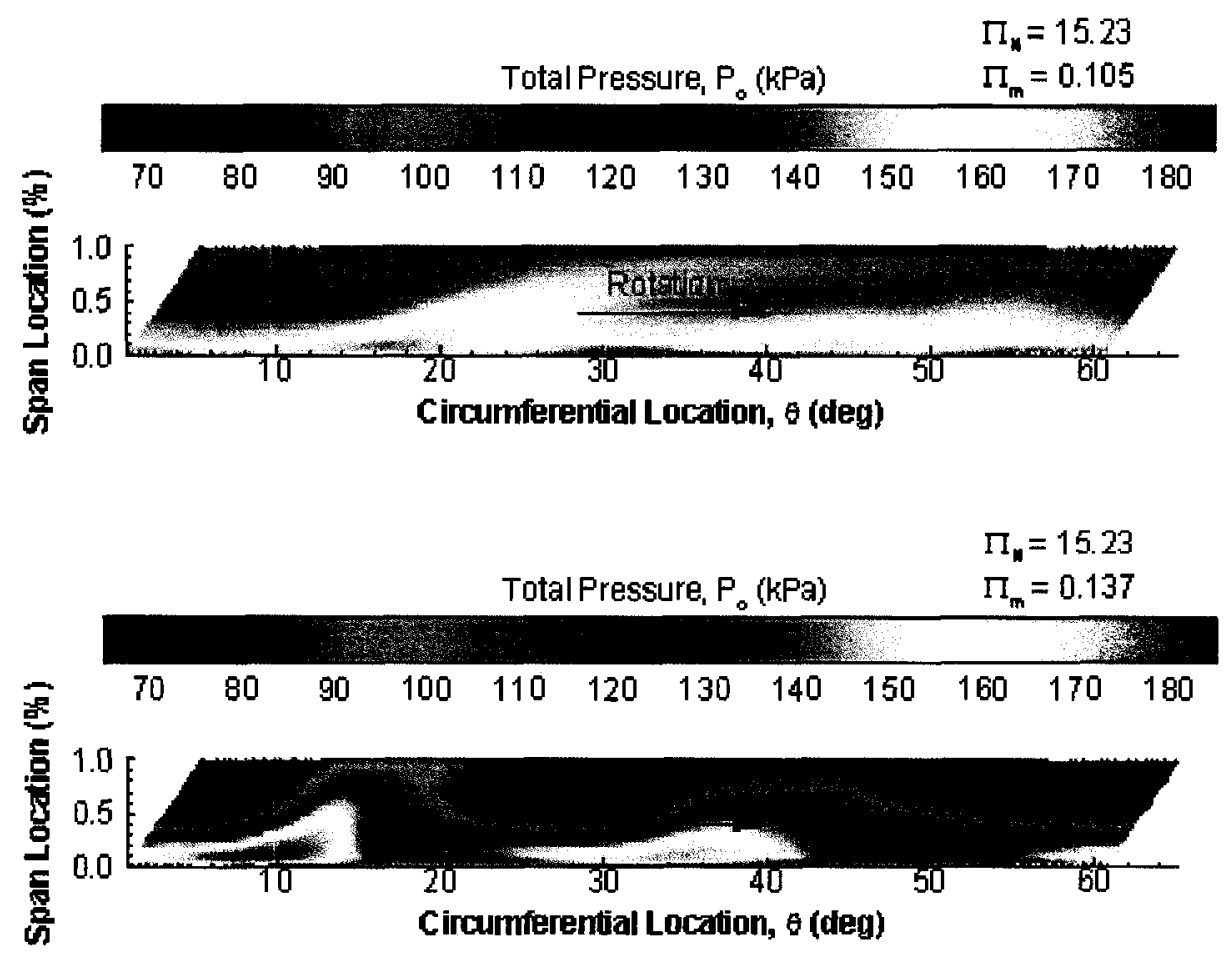

Figure 7.21: Contours of total pressure downstream of the T04E-50 impeller outlet

Figure 7.21 shows that the total pressure distribution is more uniform at the lower mass flow parameter. Nevertheless, there are significant circumferential variations in total pressure near the impeller outlet in both cases. Specifically, the two regions of high total pressure from the main and splitter blade passages are identifiable at the measurement plane but are more pronounced at the higher mass flow rate. The regions of high total pressure also contain a larger amount of the mass flow. The port on the total pressure probe spans about $3 \%$ of the passage height and is fixed at $50 \%$ span. Because the size and location of the high pressure regions change with mass flow rate their position relative to the probe port will also change. The mass-averaged total pressure in the probe area will therefore not necessarily be representative of the overall massaveraged total pressure and the relationship between the two will vary with the operating point. Thus the change in size and relative position of the high pressure regions with 
mass flow rate is a possible cause of the scatter seen in the constant speed lines shown in Section 7.4.1. Figure 7.21 also shows large spanwise variations of total pressure. This indicates that the pressure sensed by the total pressure probe can vary considerably with small variations in its spanwise location.

At the reference operating speed parameter of 15.23 , approximately 690 passages pass by each total pressure probe during the sampling time. The total pressure measurement obtained is therefore averaged in some manner depending on the response of the probe. However, it is unclear whether this is a true time average of the pressure. In addition, the location of each total pressure probe varies across the span. It is believed that the spanwise position of the probe hole is at best accurate to $\pm 1 \mathrm{~mm}$. This corresponds to an uncertainty of $\pm 20 \%$ of the nominal $50 \%$ span position. Based on these considerations, the Pitot probe measurements provide only a very approximate measure of the total pressure at that particular circumferential location.

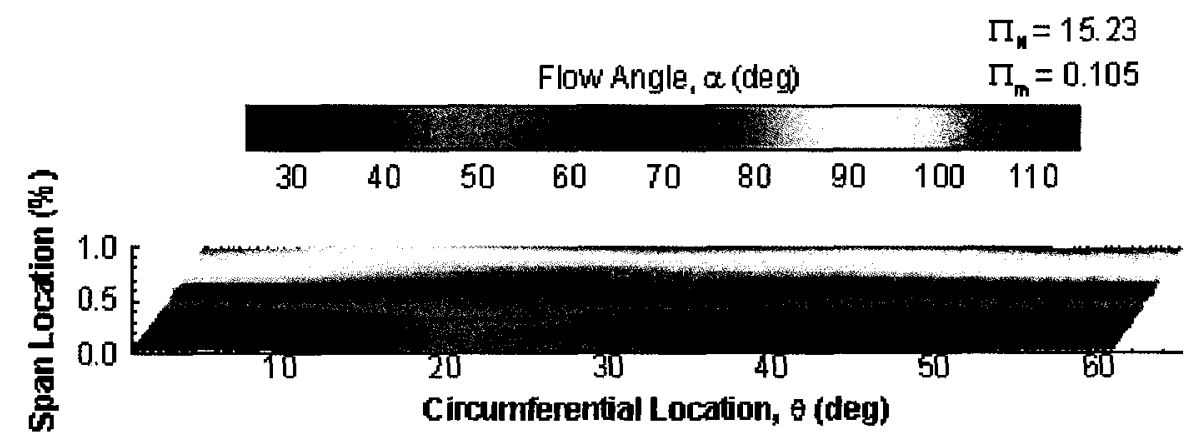

Figure 7.22: Contours of flow angle downstream of the T04E-50 impeller outlet

Figure 7.22 shows the predicted contours of flow angle downstream of the impeller outlet over one blade set spacing. As seen, there is little circumferential variation in the flow angle; however, spanwise variations are evident. This illustrates the difficulty in measuring the flow angle at the measurement plane. Within the expected uncertainty of the total pressure probe spanwise location, the uncertainty in the flow 
angle may be as much as $\pm 10^{\circ}$. This contributes to the differences in the measured circumferential variations in flow angle seen in Section 7.3.3. The flow angle distribution shows no evidence of the wakes of the main and splitter blades. Thus the mass-averaged total pressure and flow angle along the thin ring area will not be similarly influenced. This could be the cause of the different trends seen in the predicted total pressure characteristic and the flow angle variations of Figures 7.15 and 7.19 respectively.

\subsubsection{Shroud Surface Static Pressures}

The shroud surface static pressure variation predicted by ANSYS CFX is compared to the measured data in Figure 7.23. A reference operating point for each value of the speed parameter is shown. The measured data seen previously in Section 7.3.4 has been non-dimensionalized to allow comparison to the CFD predictions. The corresponding static pressure coefficient has been plotted for various streamwise

positions. As seen, the operating values of the mass flow parameter for a given speed parameter could not be matched exactly between the measurements and the predictions. The difference between the measured and predicted mass flow parameter is largest for the lowest speed parameter.

There is reasonable agreement between the measured and predicted static pressure variation for speed parameters of 15.23 and 18.35 . Additionally, the measured data for all three speed parameters show similar trends. However, the predicted static pressure variation for the lowest speed parameter does not match the other predicted pressure variations or the corresponding measured data. The reason for this is not fully understood. 


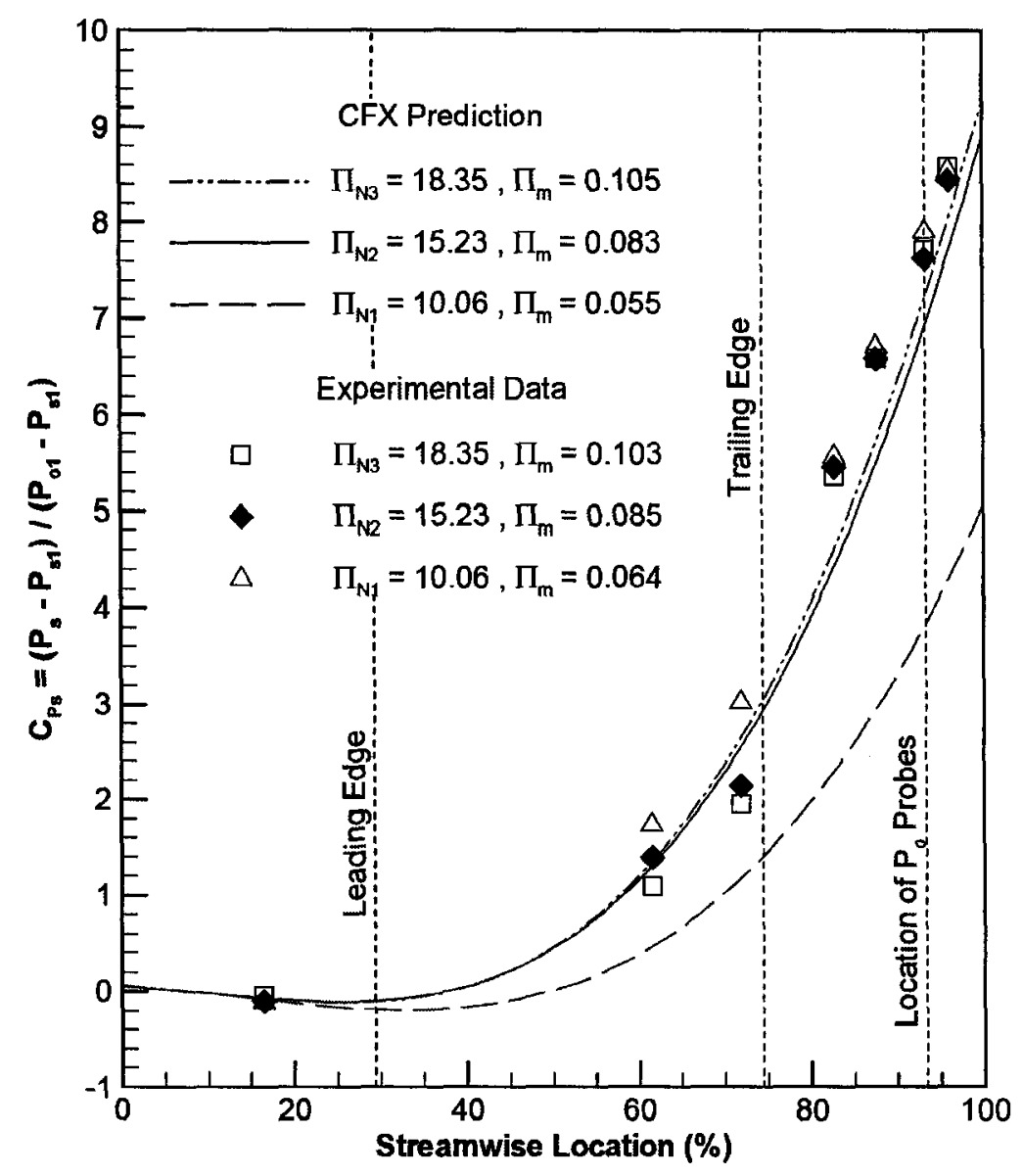

Figure 7.23: Shroud surface static pressure variation predicted by ANSYS CFX compared to measured data for the T04E-50 impeller

The predicted contours of static pressure coefficient for a single operating point and three spanwise locations are shown in Figure 7.24. There is relatively little change in the pressure contours across the span. This implies that static pressure information measured at the shroud, or $100 \%$ span, could be used to infer the pressure variation inside the blade passage. However, this would require fast response, flush mounted pressure transducers capable of providing instantaneous pressure measurements at a given location. 

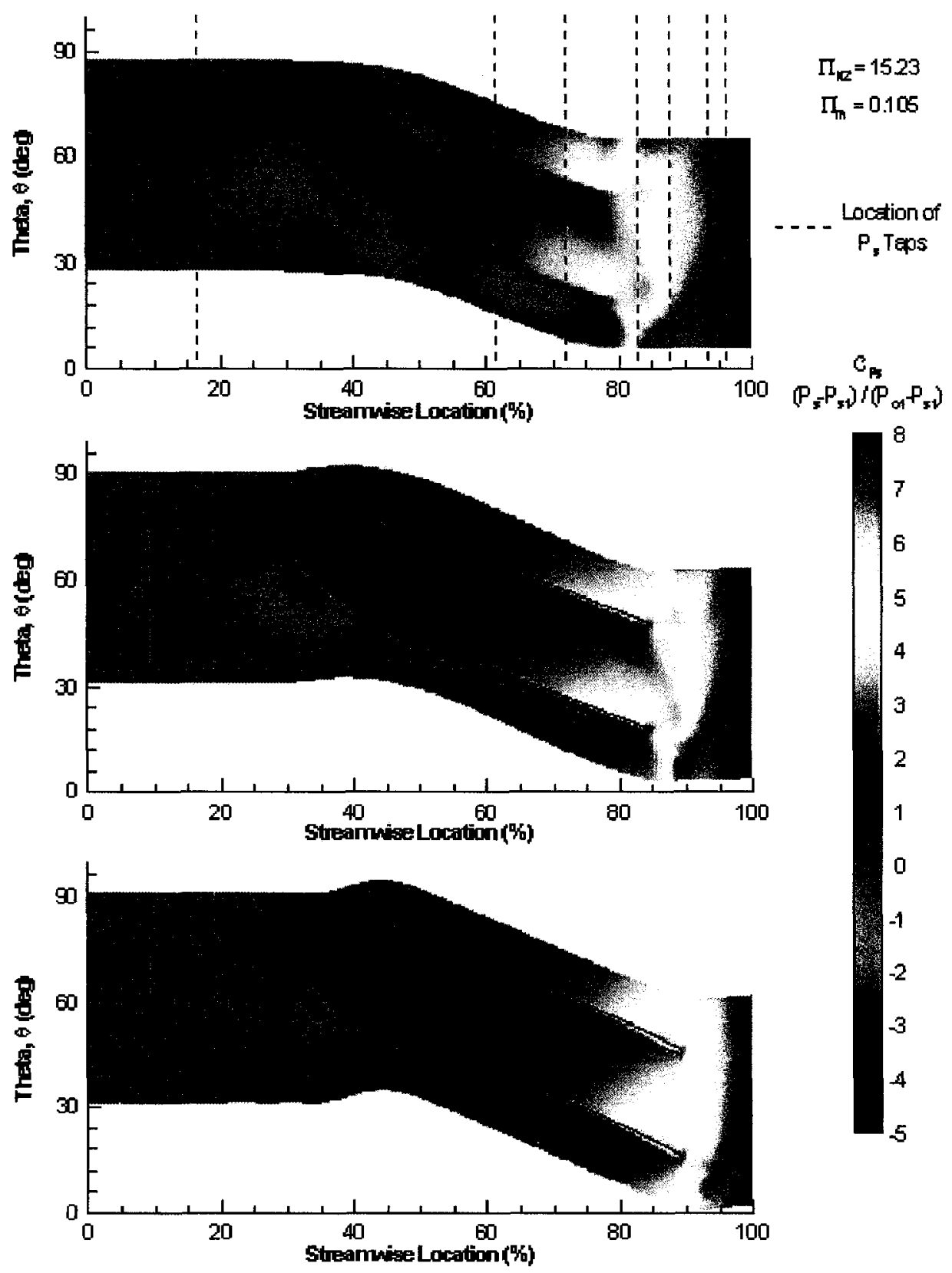

Figure 7.24: Contours of the static pressure coefficient, $C_{P s}$ at multiple span locations for the T04E-50 impeller 


\subsection{TEST RIG COMPRESSOR PERFORMANCE PREDICTIONS USING ANSYS CFX}

\subsection{INTRODUCTION}

The design and preliminary aerodynamic performance predictions of the rotating test rig compressor impeller were conducted using CFX-BladeGen. These results, detailed in Section 6.2, provided a manufacturable impeller ready for experimental testing and detailed CFD predictions. As mentioned previously time constraints prevented the collection of the experimental performance data for this impeller. Instead the aerodynamic performance estimates were limited to detailed CFD predictions using ANSYS CFX. The current chapter discusses these results.

The accuracy of the BladeGen+ performance predictions is limited by limitations of the computational software. ANSYS CFX should provide more accurate aerodynamic predictions than BladeGen+ because of its ability to generate more detailed grids and model the tip clearance gap. In addition, the SST turbulence model was selected because it is more appropriate for complex flows than the zero-equation model available in BladeGen+. The test rig impeller performance characteristics predicted by ANSYS CFX are compared against those predicted by BladeGen+ in Section 8.2. More detailed aerodynamic predictions by CFX are also shown and include spanwise contour plots and blade loading distributions. In addition, the influence of tip clearance on the impeller performance characteristics was investigated.

The original intent of this study was to provide experimental data for the new test rig impeller. To allow experimental testing of the existing rotating test rig, a compressor casing and volute were also designed. This process yielded a solids model of the volute and allowed aerodynamic predictions of the complete test rig compressor stage to be 
made using ANSYS CFX. The predicted complete stage performance is discussed in Section 8.3. The performance of the volute and its effect on the impeller characteristics was also investigated and is discussed in Section 8.4.

\subsection{Test Rig ImPeller PeRformance Predictions}

\subsubsection{Performance Characteristics}

The impeller total pressure ratio and isentropic efficiency characteristics as predicted by CFX are shown in Figures 8.1 and 8.2 and are compared to those predicted by BladeGen+. The reference operating point indicated on both figures corresponds to the conditions for which the more detailed aerodynamic predictions were obtained. These will be discussed later. In addition to the design operating speed of 110,000 RPM $\left(\Pi_{N}=31.59\right)$, a second constant speed operating line of $60,000 \mathrm{RPM}\left(\Pi_{N}=17.23\right)$ is shown. The performance was predicted at this off-design rotational speed because it is believed that future experimental testing will not be possible at the design rotational speed. The working fluid of the new test rig impeller is $\mathrm{CO}_{2}$ and thus, all computations were performed using $\mathrm{CO}_{2}$ as the working fluid.

Figure 8.1 and 8.2 show that the predicted choking mass flow rate is higher for the CFX computations than for the BladeGen+ results. This is due to geometric differences in the two computational models. The two models have identical rotor geometries, with a blade height of $3 \mathrm{~mm}$ at the trailing edge. However, the CFX model includes an additional $1 \mathrm{~mm}$ clearance between the rotor tip and the shroud, for a total passage height of $4 \mathrm{~mm}$ at the outlet. Thus the tip clearance spans $25 \%$ of the overall passage height or $33 \%$ of the blade height. Since tip clearance cannot be modelled in BladeGen ${ }^{+}$, its calculations were made for a passage height corresponding to the span of the rotor blades ( $3 \mathrm{~mm}$ at the outlet). This also had the effect of reducing the channel cross-sectional area in the throat region (near the leading of the splitter blade), and thus 
reducing the choking mass flow rate for the BladeGen+ computations. In retrospect, it would have been preferable to perform the BladeGen+ calculations for the full, actual passage height.

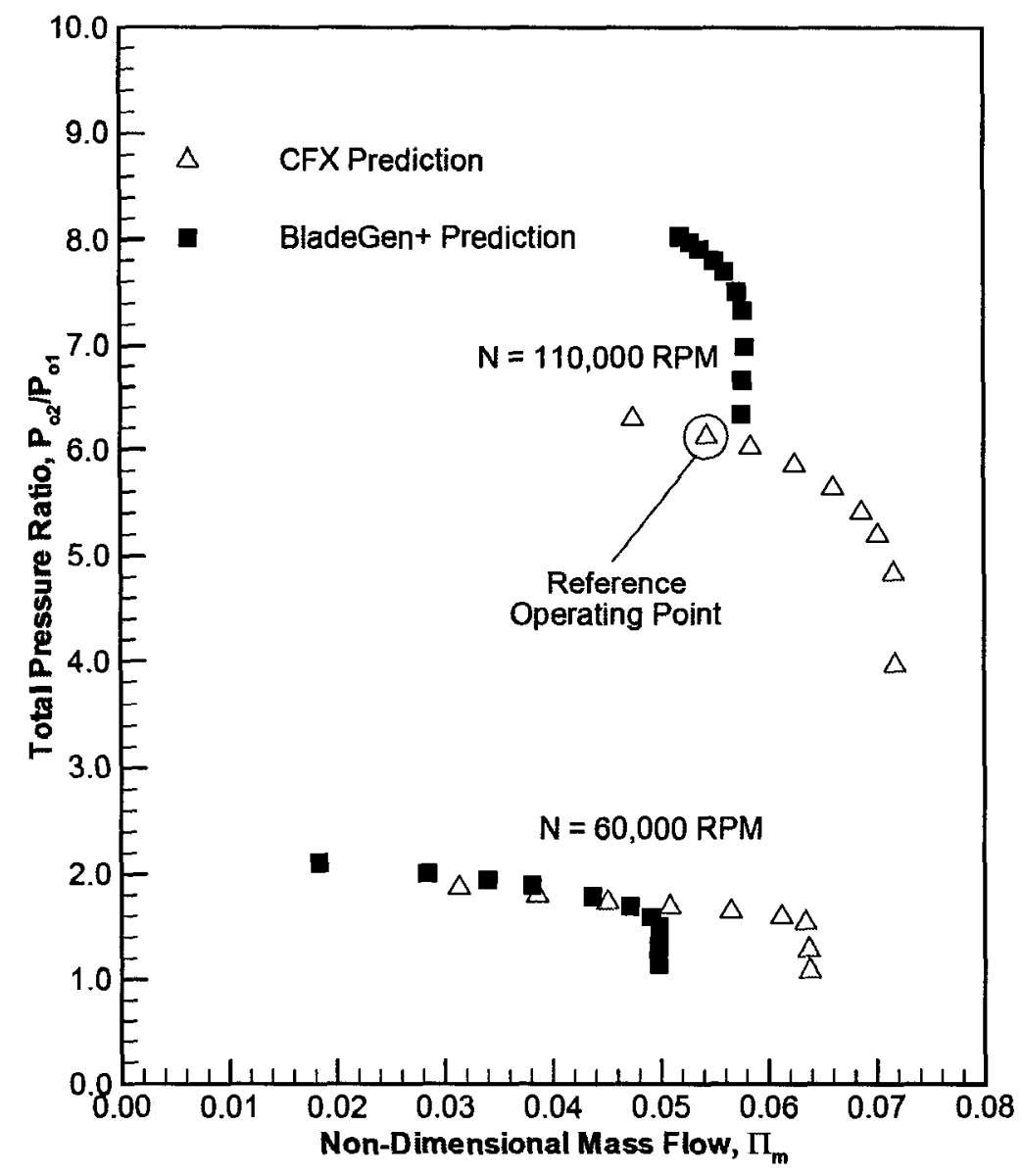

Figure 8.1: Total pressure characteristic of the test rig impeller predicted by ANSYS CFX

Figure 8.1 also shows a lower pressure rise predicted by CFX than predicted by BladeGen+ for a given non-dimensional mass flow rate. Again this is a result of the modelled tip clearance. The resulting tip clearance leakage flow increases total pressure losses and subsequently reduces the isentropic efficiency as seen in Figure 8.2. As expected the leakage flow has a greater effect on the impeller performance at higher 
rotational speeds. The leakage flow also has the potential to cause trailing edge separation near the shroud and further reduce the pressure rise and efficiency. The effect of the tip clearance is investigated further in Section 8.2 .3 by examining the performance characteristics predicted by CFX for zero tip clearance.

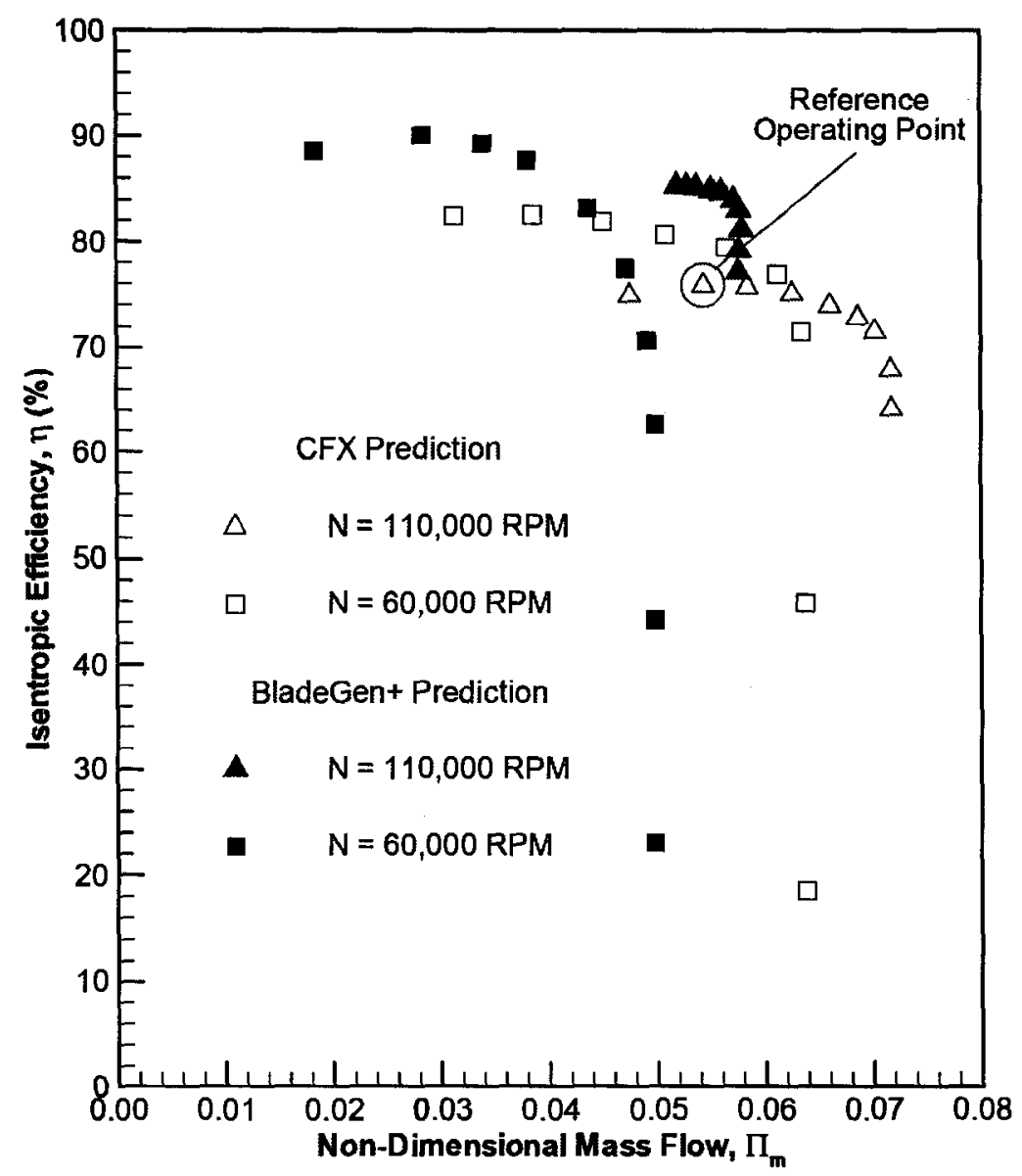

Figure 8.2: Efficiency characteristic of the test rig impeller predicted by ANSYS CFX

\subsubsection{Detailed Aerodynamic Performance}

The predictions obtained with CFX can also be used to examine the detailed aerodynamics of the machine. The reference operating point examined is indicated on Figures 8.1 and 8.2 . 


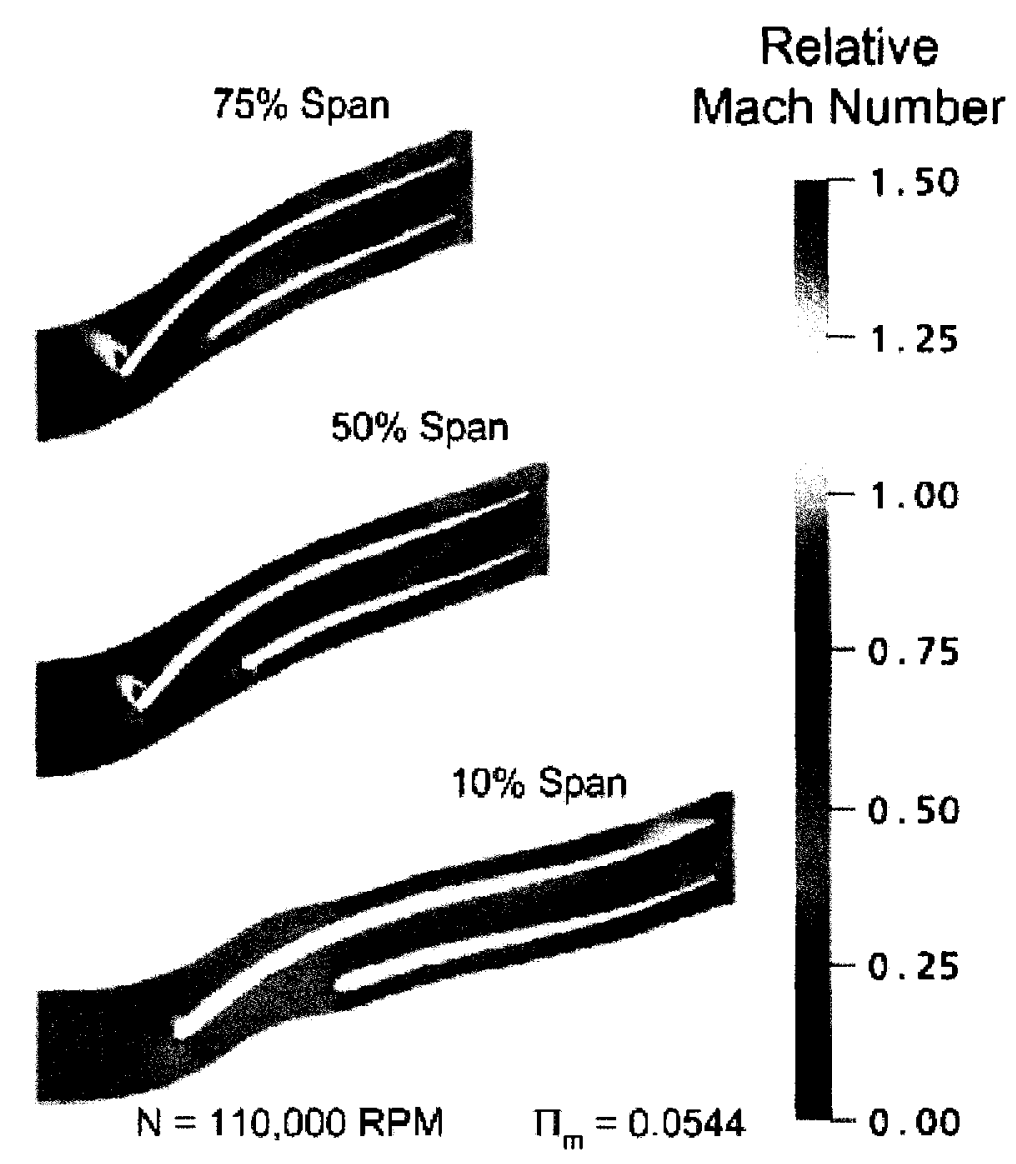

Figure 8.3: Contours of relative Mach number at multiple spans of the test rig impeller predicted by ANSYS CFX

Figure 8.3 shows the contours of relative Mach number at three constant span locations in the test rig impeller. Larger spanwise position corresponds to a plane closer to the shroud. With the tip clearance modelled, the $75 \%$ spanwise position corresponds to the vane height at the trailing edge. As seen, there is a region of low velocity flow on the suction side of both blades near the trailing edge at $75 \%$ span. This region is also present at $50 \%$ span but only on the splitter blade. It is believed that this may be separation induced by the tip clearance leakage flow from the pressure side to the suction side of the blades. It is unclear why the main blade is seemingly less affected by the leakage flow. 
The leading edge incidence on the main blade is approximately $10^{\circ}$ at $10 \%$ and $50 \%$ span. At $75 \%$ span the incidence is approximately $6^{\circ}$. As seen in Figure 8.3 this results in a region of over speed near the leading edge of the main blade at these spanwise locations. There is also evidence of low velocity fluid at $75 \%$ span slightly downstream of the over speed. The local Mach numbers reached a maximum of approximately 1.4 near the leading edge and thus the region of over speed is expected to end in a shock wave. It is therefore possible that the low velocity fluid is evidence of a shock-induced separation bubble.

The contours of relative total pressure at three constant spanwise locations are shown in Figure 8.4. As seen there is a large total pressure loss half way through the passage at $75 \%$ span. A similar total pressure loss is seen further downstream as the span is reduced. Again this is likely due to flow separation caused by the tip clearance leakage flow. Because the vane height decreases downstream, the constant $1 \mathrm{~mm}$ tip clearance is a larger percentage of the flow area near the trailing edge and therefore has a greater influence in this region. Also seen is a small region of low relative total pressure near the leading edge at $75 \%$ span. This is further evidence of a possible shock-induced separation bubble.

Figure 8.4 also shows the boundary layer growth predicted by CFX. Although it is not very clear from the figure shown, it is evident that the growth is occurring much more slowly than predicted by BladeGen+ (Fig. 6.11). The subsequent total pressure losses due to viscous effects predicted by CFX are therefore less than those predicted by BladeGen+. The boundary layer growth predicted by CFX is expected to be more accurate because of the use of the more sophisticated SST turbulence model in CFX. 


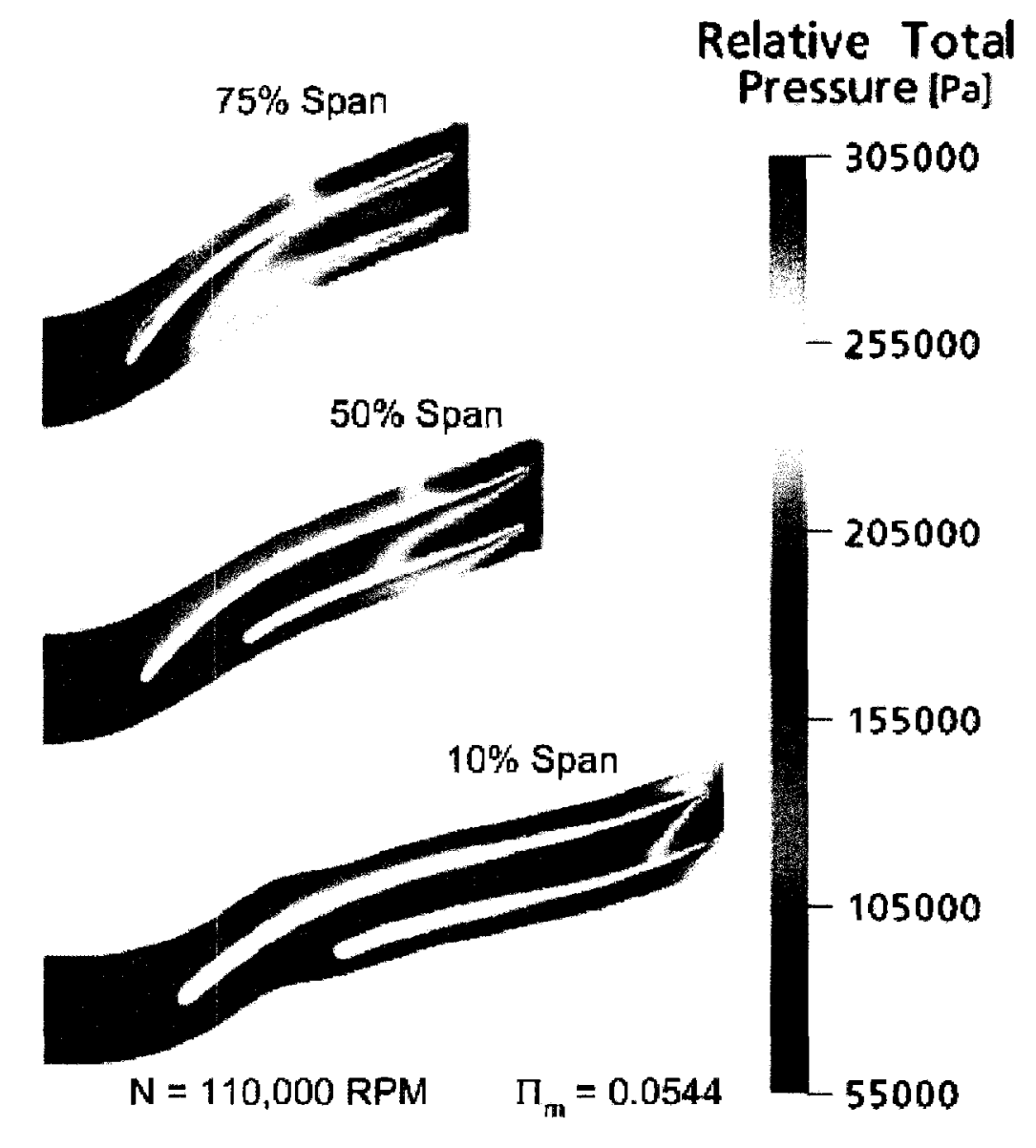

Figure 8.4: Contours of relative total pressure at multiple spans of the test rig impeller predicted by ANSYS CFX

The blade aerodynamic loading distribution for the test rig impeller was also predicted by ANSYS CFX. Figure 8.5 shows the loading distribution at mid-span for the main and splitter blades. As seen, the aerodynamic loading is highest after $60 \%$ of the streamwise distance along the main blade. The loading distribution remains smooth along the main blade suction surface and this suggests that the flow remains attached. The aerodynamic loading of the splitter blade is significantly less than the main blade beyond about $60 \%$ streamwise distance. This lower loading is the result of higher static pressures on the suction side of the splitter blade than on the main blade. Also seen in Figure 8.5 are over speeds resulting from the leading edge incidence on the main and splitter blades. The loading distribution of the main blade shows that work is being 
transferred to the fluid along the entire $50 \%$ span of the blade. However, the over speed on the splitter blade creates a short region of reversed loading near the leading edge.

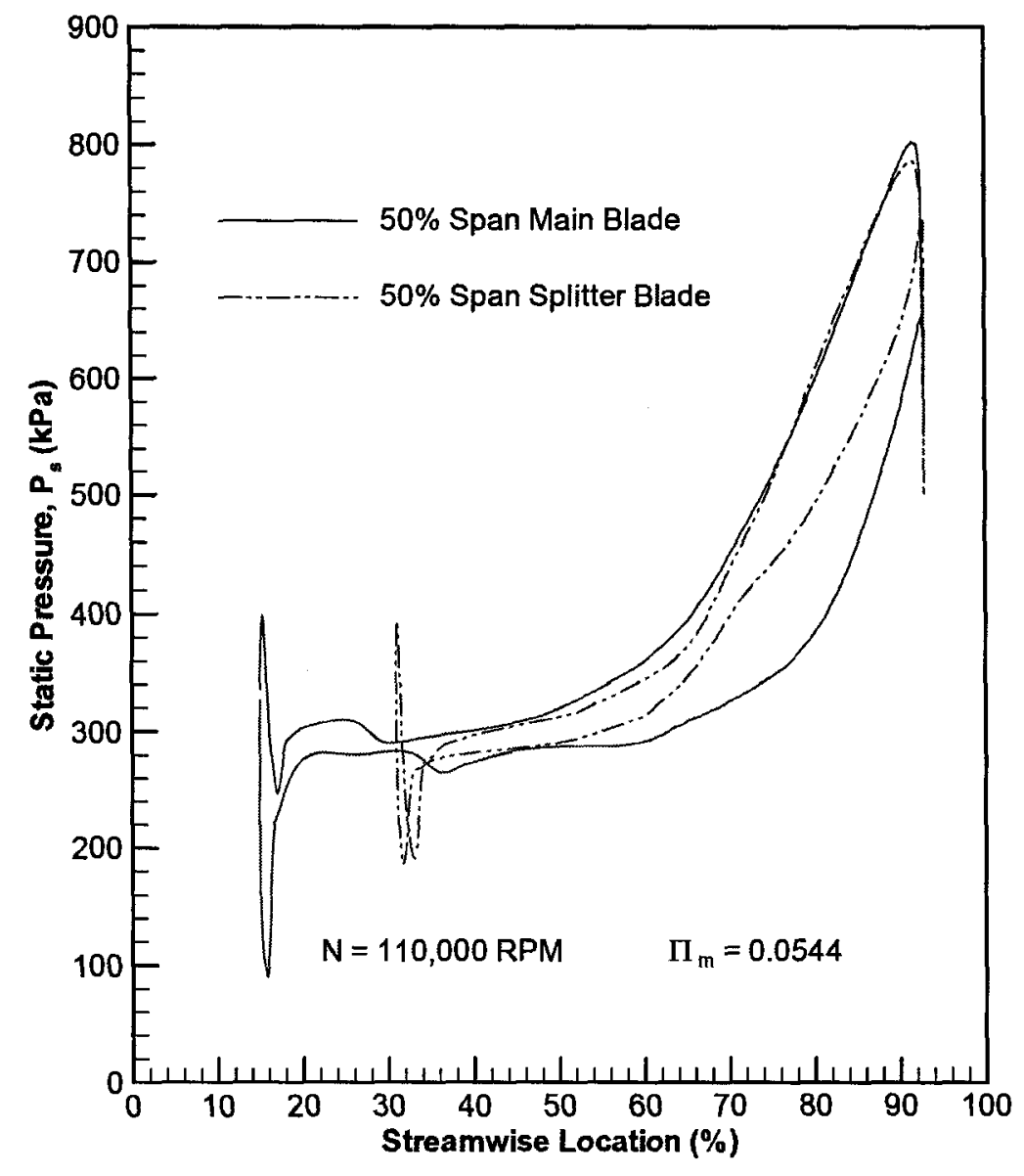

Figure 8.5: Blade aerodynamic loading at mid span of the test rig impeller predicted by ANSYS CFX

Figure 8.6 compares the aerodynamic loading distribution for the main blade at 10 $\%, 50 \%$ and $75 \%$ span. The effect of the leading edge incidence on the blade loading can be seen for all three spanwise positions. At $10 \%$ and $50 \%$ span the loading distribution remains smooth beyond approximately $35 \%$ streamwise position. However, at $75 \%$ span there is a significant difference in the static pressure along the pressure side after $70 \%$ streamwise position. The vane height near the trailing edge corresponds to the 
$75 \%$ spanwise position. Thus the pressure disturbance seen in the loading is the result of the data plane approaching the tip of the blade and the leakage flow over the tip.

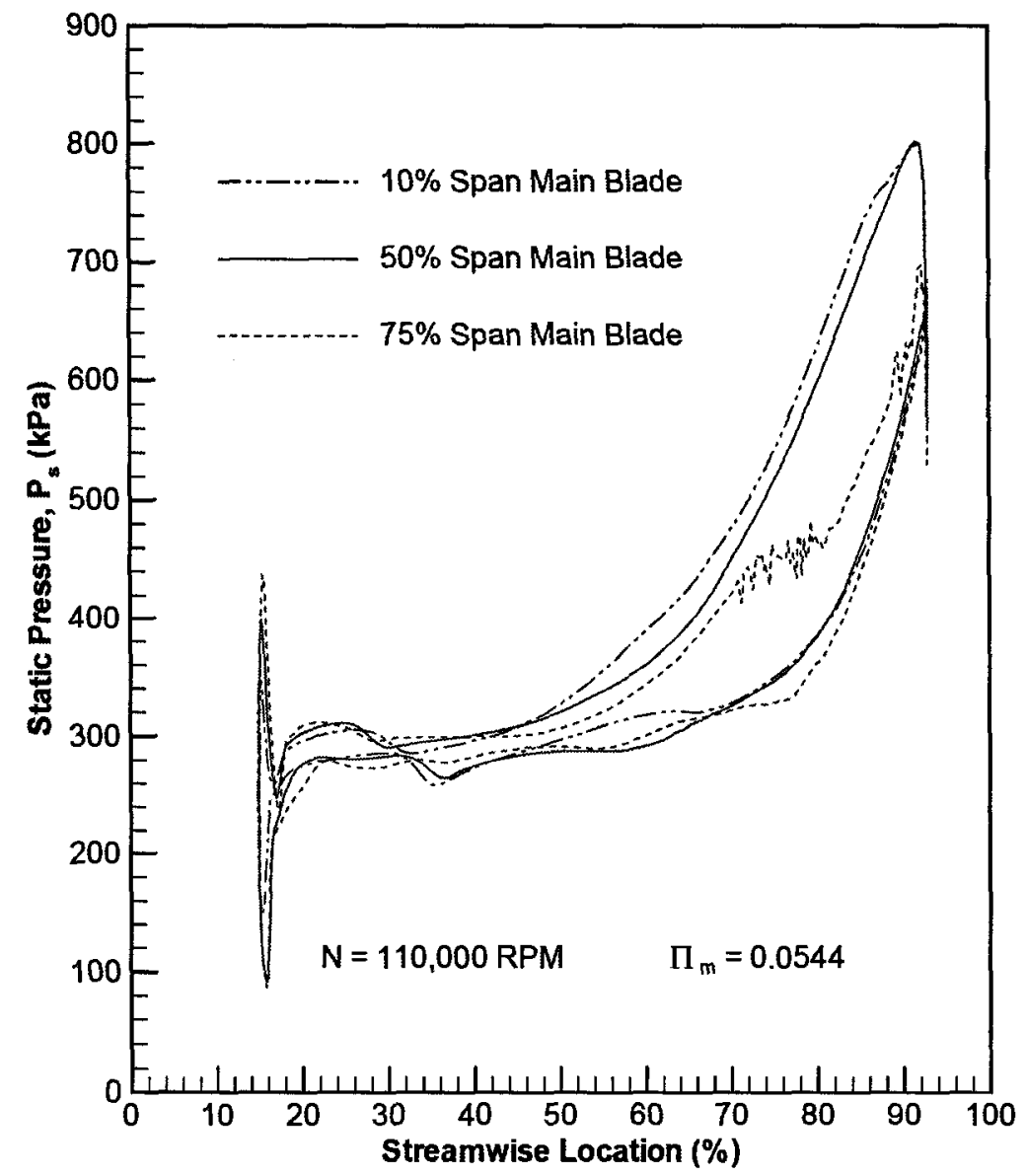

Figure 8.6: Blade aerodynamic loading at multiple spans of the test rig impeller main blade predicted by ANSYS CFX

\subsubsection{Effect of Tip Clearance on Impeller Performance}

The comparison of the impeller performance characteristics predicted by BladeGent and ANSYS CFX were performed with the same impeller geometry. However, the BladeGen+ calculations were performed with zero tip clearance and the overall passage height of the BladeGen+ calculations is therefore equal to the vane height 
along the entire passage length ( $3 \mathrm{~mm}$ at the trailing edge). The CFX calculations were performed with the same impeller geometry but included the specified $1 \mathrm{~mm}$ tip clearance. The resulting passage height of the CFX calculations was therefore a constant $1 \mathrm{~mm}$ larger than the BladeGen+ predictions. At the trailing edge, this corresponds to a passage height of $4 \mathrm{~mm}$ and thus the tip clearance spans $25 \%$ of the flow passage. The size of the tip clearance relative to the blade height at the trailing edge is significantly larger than would be expected for a well designed compressor and was the result of manufacturing constraints in the present case. It was therefore decided to investigate the effect of the tip clearance on the impeller performance. To do so, the geometry used in the BladeGen+ predictions was repeated in ANSYS CFX. It was then possible to confirm that the differences between the CFX and BladeGen+ predictions are mainly due to the differences in the passage height and the effect of tip leakage.

Figure 8.7 and 8.8 show the performance characteristics of the test rig impeller for the two geometries as predicted by ANSYS CFX. Also shown are the original zero-tipclearance predictions of BladeGen+. To reduce clutter the BladeGen+ data points have been represented with a solid line. As can be seen the total pressure rise and isentropic efficiency predicted by CFX for zero tip clearance $\left(\tau / b_{\mathrm{TE}}=0 \%\right)$ is greater than those predicted by BladeGen ${ }^{+}$, particularly at higher rotational speeds. This is probably partly due to the turbulence model used in BladeGen+ and its tendency to over predict the total pressure losses. The CFX predicted pressure rise and efficiency with zero tip clearance modelled $\left(\tau / b_{\mathrm{TE}}=0 \%\right)$ is also greater than the pressure rise and efficiency predicted using CFX with the tip clearance modelled $\left(\tau / b_{\mathrm{TE}}=25 \%\right)$. Again this is expected and is due to the tip leakage and its corresponding total pressure losses. As mentioned before, the choking mass flow rate is greater with the tip clearance modelled and is a result of the increased throat area. It is therefore clear that the performance of the test rig impeller is strongly influenced by the tip clearance. 


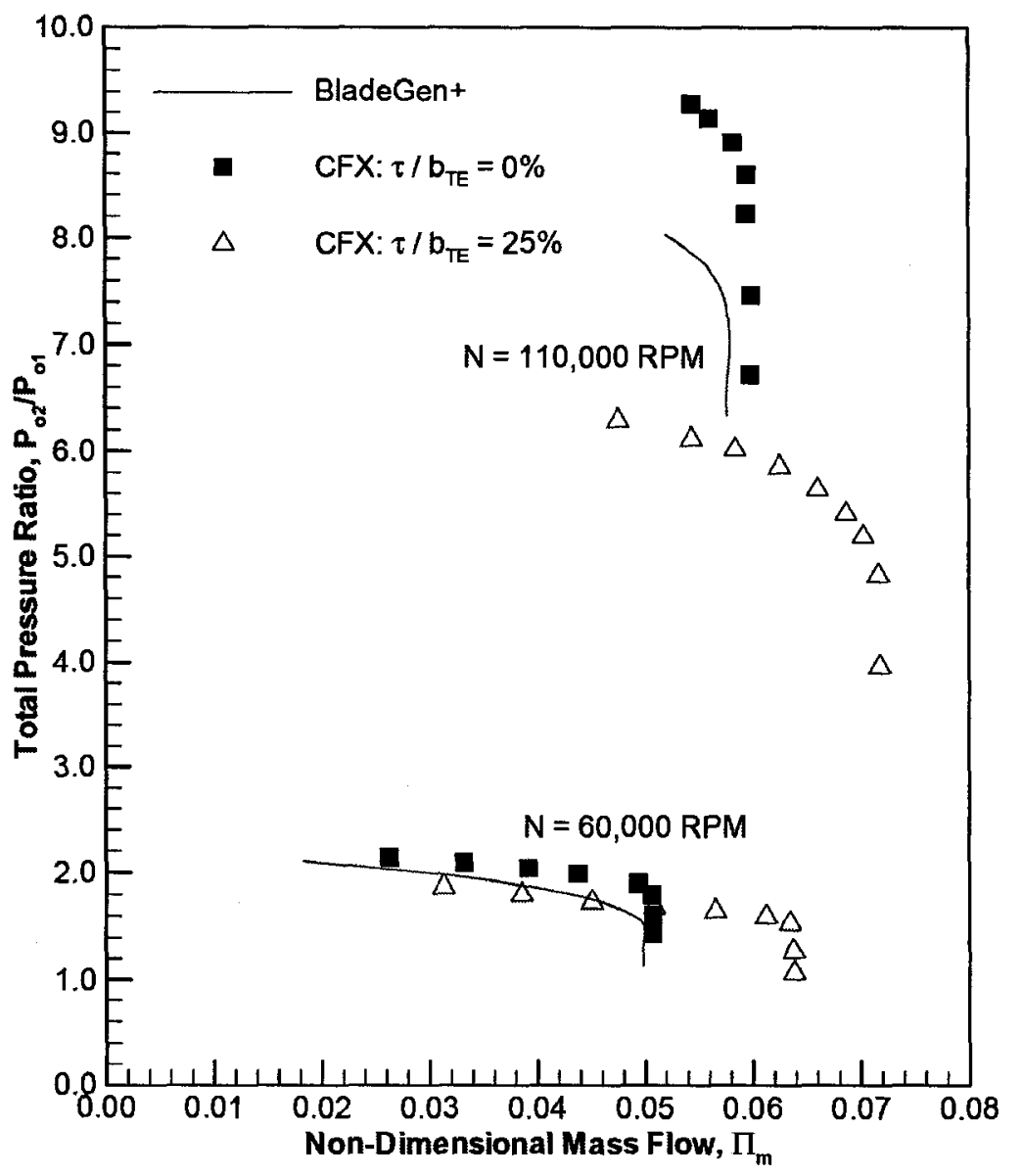

Figure 8.7: Effect of tip clearance on the predicted pressure rise characteristic of the test rig impeller 


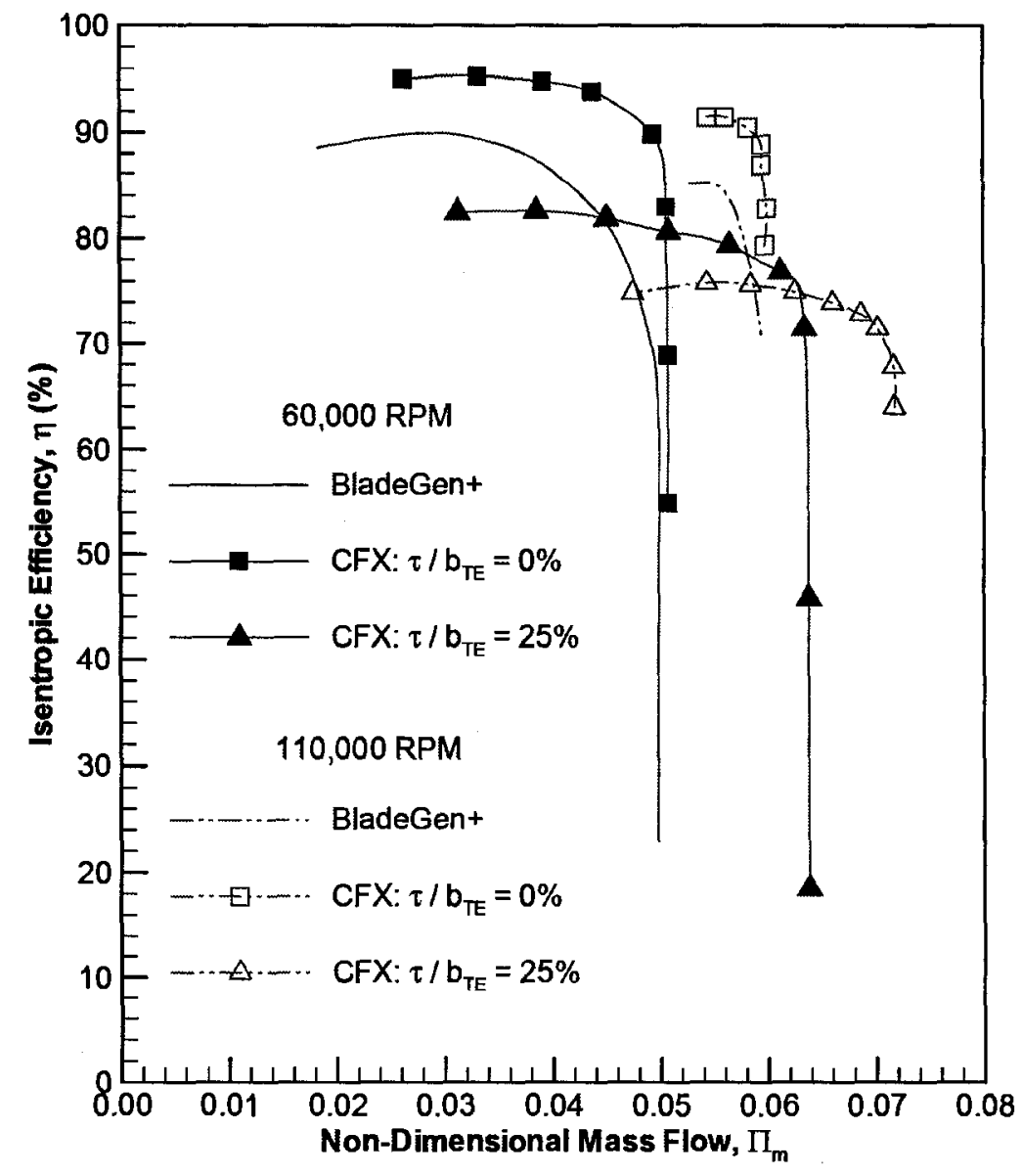

Figure 8.8: Effect of tip clearance on the predicted efficiency characteristic of the test rig impeller

\subsection{Stage Performance Predictions}

\subsubsection{Stage Performance Characteristics}

For future testing in the rotating test rig a volute and compressor casing for the test rig compressor were also designed and manufactured. This section therefore presents the predicted performance for the complete stage. 
The complete stage of the test rig compressor was modelled and solved using ANSYS CFX. In axial compressor design a complete stage consists of both a rotor and stators. In the case of a centrifugal compressor the diffuser and possibly a volute function as the stators. Due to the complexity of the geometry of the test rig compressor the diffuser was not separately modelled in CFX. Instead the impeller domain was extended beyond the trailing edge to approximately mid way through the vaneless diffuser. The remaining diffuser passage was obtained by extending the inlet of the volute domain upstream. The domain modelled within CFX therefore consists of only two components, the impeller and the volute. For this reason, the complete stage of the test rig impeller is stated as consisting of the impeller and volute, corresponding to the two domains used in CFX. As a result of the models used, the diffuser passage contains both stationary and rotating walls. The shroud wall is stationary for the entire radial length of the diffuser. However the hub wall, which extends from the impeller through half the radial length of the diffuser, is specified as a rotating wall. In retrospect it might have been better to model the hub surface beyond the trailing edge as a separate, stationary wall.

The stage total pressure ratio characteristic of the test rig compressor is shown in Figure 8.9. The same rotational speeds were examined as for the impeller-only calculations presented in Section 8.2. For comparison, the impeller-only performance predictions are also shown. The reference operating point corresponds to the conditions used to obtain more detailed aerodynamic predictions of the stage and to obtain the performance of the volute, as will be discussed later.

As seen in Figure 8.9 the peak total pressure rise predicted for the complete stage is lower than that for the impeller alone. This is expected and is the result of total pressure losses in the downstream diffuser and volute. The difference in the stage and impeller peak pressure ratio is greater at higher rotational speeds and is likely because of the increased velocities in the volute and the consequently increased total pressure losses. 


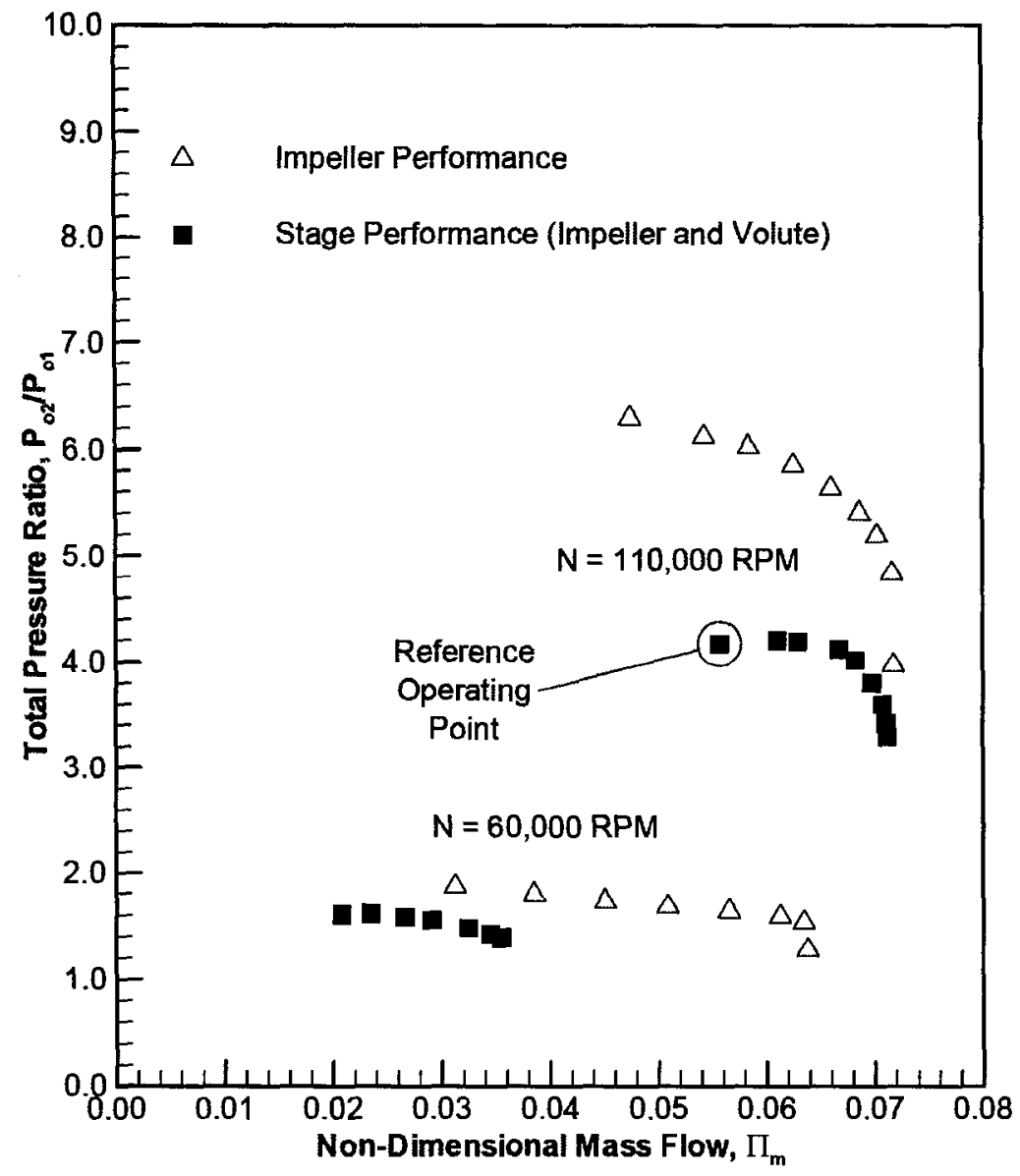

Figure 8.9: Total pressure ratio characteristic of the test rig impeller and stage predicted by ANSYS CFX

The isentropic efficiency characteristic of the complete test rig compressor stage is shown in Figure 8.10. Again the peak efficiency is higher for the impeller only than the complete stage and is due to total pressure losses through the volute and their effect on the efficiency of the complete stage. Figure 8.10 also shows a higher peak efficiency for both the impeller and stage at the lower rotational speed. This is again believed to be the result of reduced velocities within the volute and thus lower total pressure losses at the lower rotational speed. The effect of the tip leakage within the impeller may also be reduced at lower rotational speeds. 


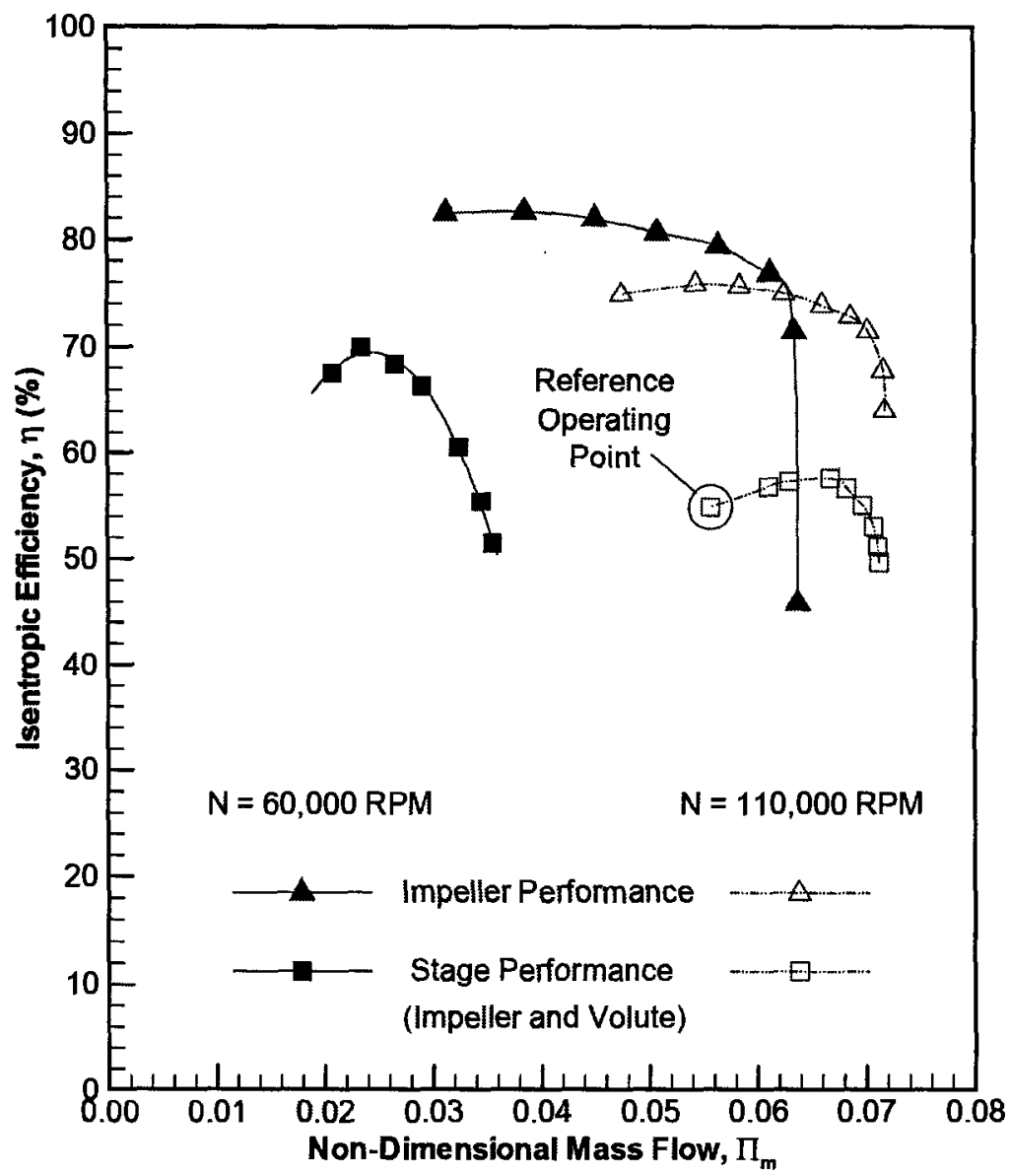

Figure 8.10: Efficiency characteristic of the test rig impeller and stage predicted by ANSYS CFX

\subsubsection{Constant Span Contour Plots}

Contour plots of predicted relative Mach number and relative total pressure for the impeller within the complete stage have been plotted from the CFX predictions. The data were obtained from the results at the same reference operating points shown in Figures 8.9 and 8.10 .

Figure 8.11 shows the relative Mach number contours on three constant spanwise planes. The blades labelled $\mathrm{A}$ through $\mathrm{D}$ are the source of the blade loading plots 
discussed later. Since the computational domain included the tip clearance, the plane at $75 \%$ span corresponds to the vane height near the trailing edge. Figure 8.11 shows large circumferential variations of the relative Mach number. As seen a low velocity region is present between the $90^{\circ}$ and $270^{\circ}$ positions and the remaining half of the circumference has regions of higher velocities. One such region of high velocity fluid is near the trailing edge, between the $315^{\circ}$ and $45^{\circ}$ positions, on the blade suction surface, at $10 \%$ span. This region corresponds to the region within the volute with the smallest crosssectional area. The high Mach numbers are believed to be a result of the volute's inability to swallow the flow uniformly around the circumference, as discussed later in Section 8.4. Thus the performance of the impeller seems to be affected significantly by the volute. The region of low velocity fluid near the trailing edge at $75 \%$ span is a result of the tip clearance and the corresponding leakage flow.

Also shown in Figure 8.11 is a region of high velocities near the leading edge of the main and splitter blades, around half the circumference, on all three constant span planes. The magnitude of the over speed is similar to that seen in the impeller without the volute as discussed in Section 8.2.2. However, the over speed shown in Figure 8.11 occurs on both the pressure and suction side of the blade. The average incidence at the leading edge is approximately $7^{\circ}$ and is a potential cause of the suction side over speed. However, positive incidence should not result in the local over speed seen on the pressure side. The leading edge region of the main and splitter blades is the minimum area section within the blade passage. Therefore, the high local Mach numbers may also be due to greater amounts of mass flow passing through the blade passages within the high velocity region. Again this is believed to be an effect of the volute on the performance of the impeller. 


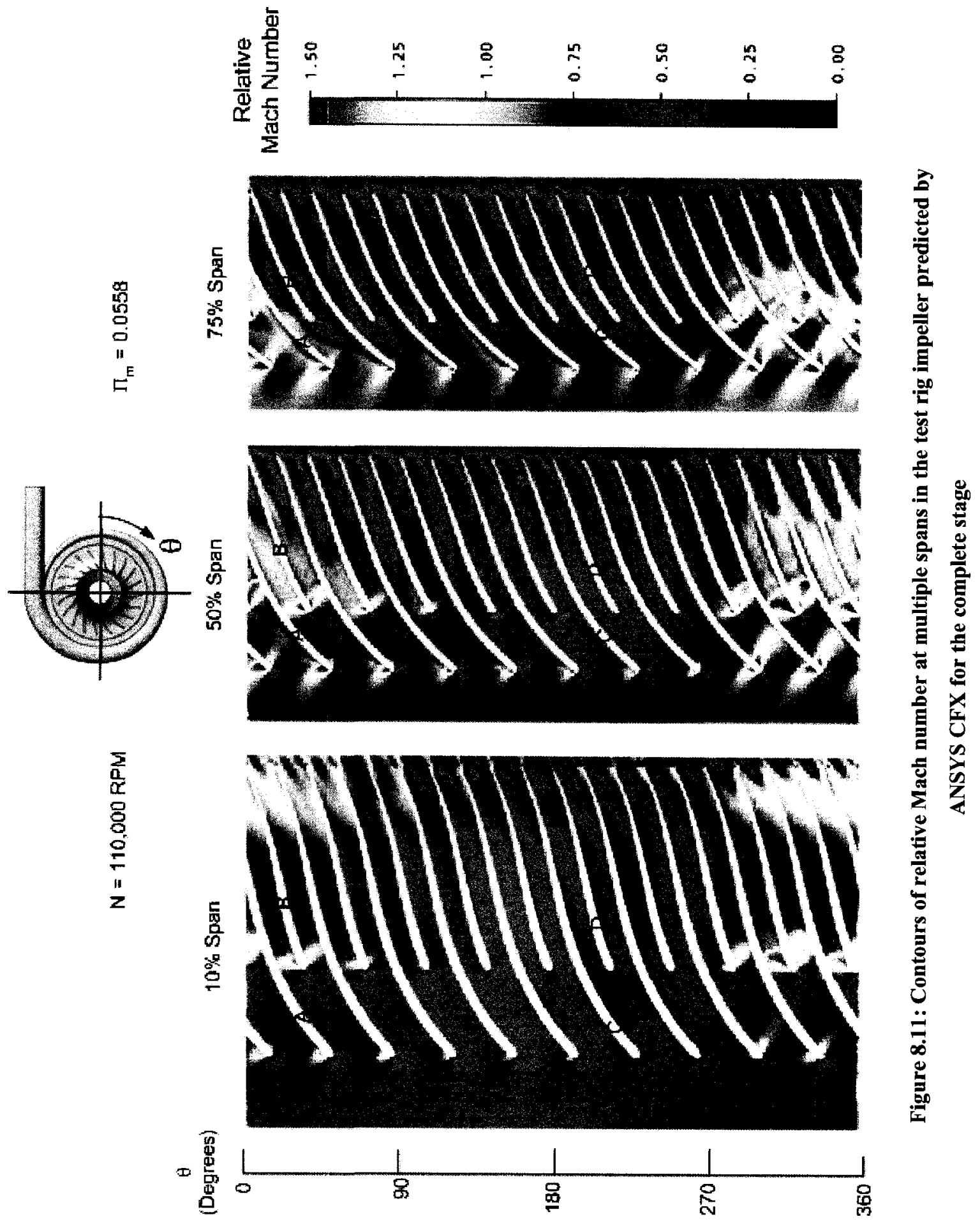


The contours of relative total pressure at three constant span planes are shown in Figure 8.12. Again the circumferential non-uniformity is evident, particularly at the larger spans. As mentioned, the $75 \%$ constant span plane corresponds to the vane height near the trailing edge. It is therefore likely that some of the total pressure losses seen at $75 \%$ span are the result of the tip leakage flow. Also seen are small regions of reduced total pressure near the leading edge on the suction surface of several main blades at $50 \%$ and $75 \%$ span. The reduced total pressure is likely the result of the positive incidence and a resulting separation of the flow from the suction surface. In all instances the flow appears able to re-attach, creating a small separation bubble. The local Mach number exceeds 1.0 upstream of all the separation bubbles. It is therefore possible that the separation bubbles are shock-induced. 
象
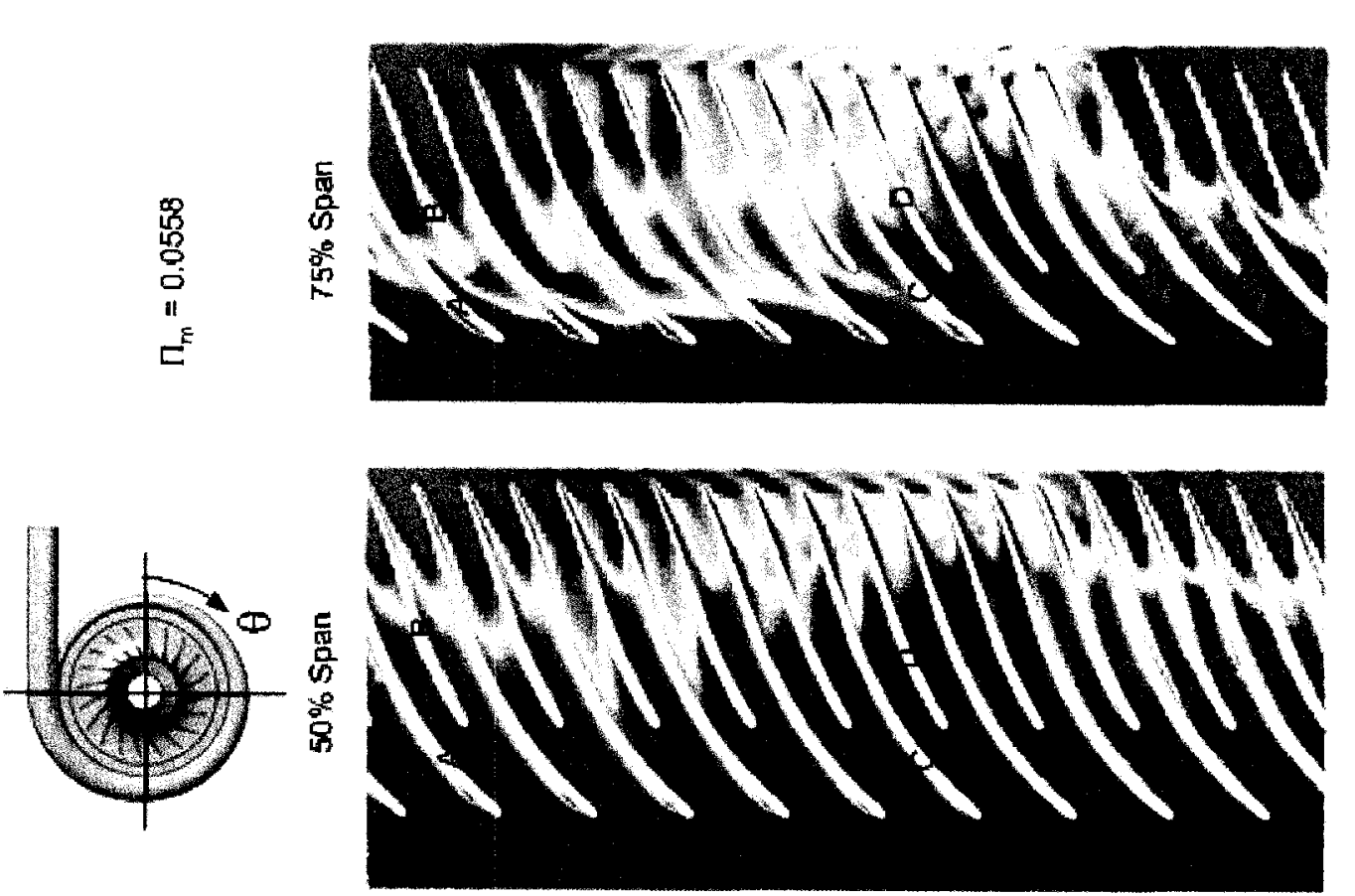

$\frac{5}{2}$
$\frac{1}{4}$
8
8
8
$\frac{5}{5}$
11
$z$
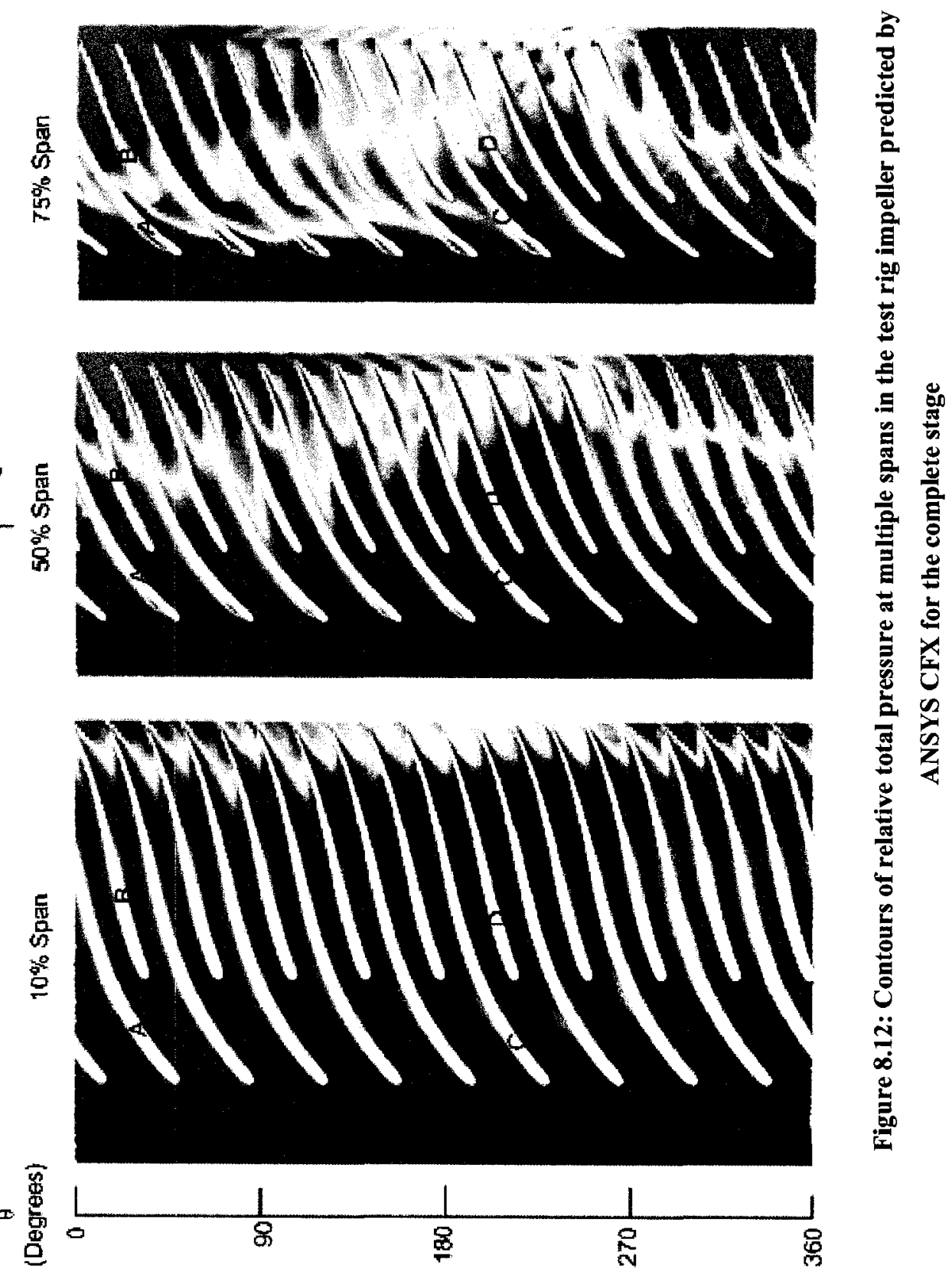


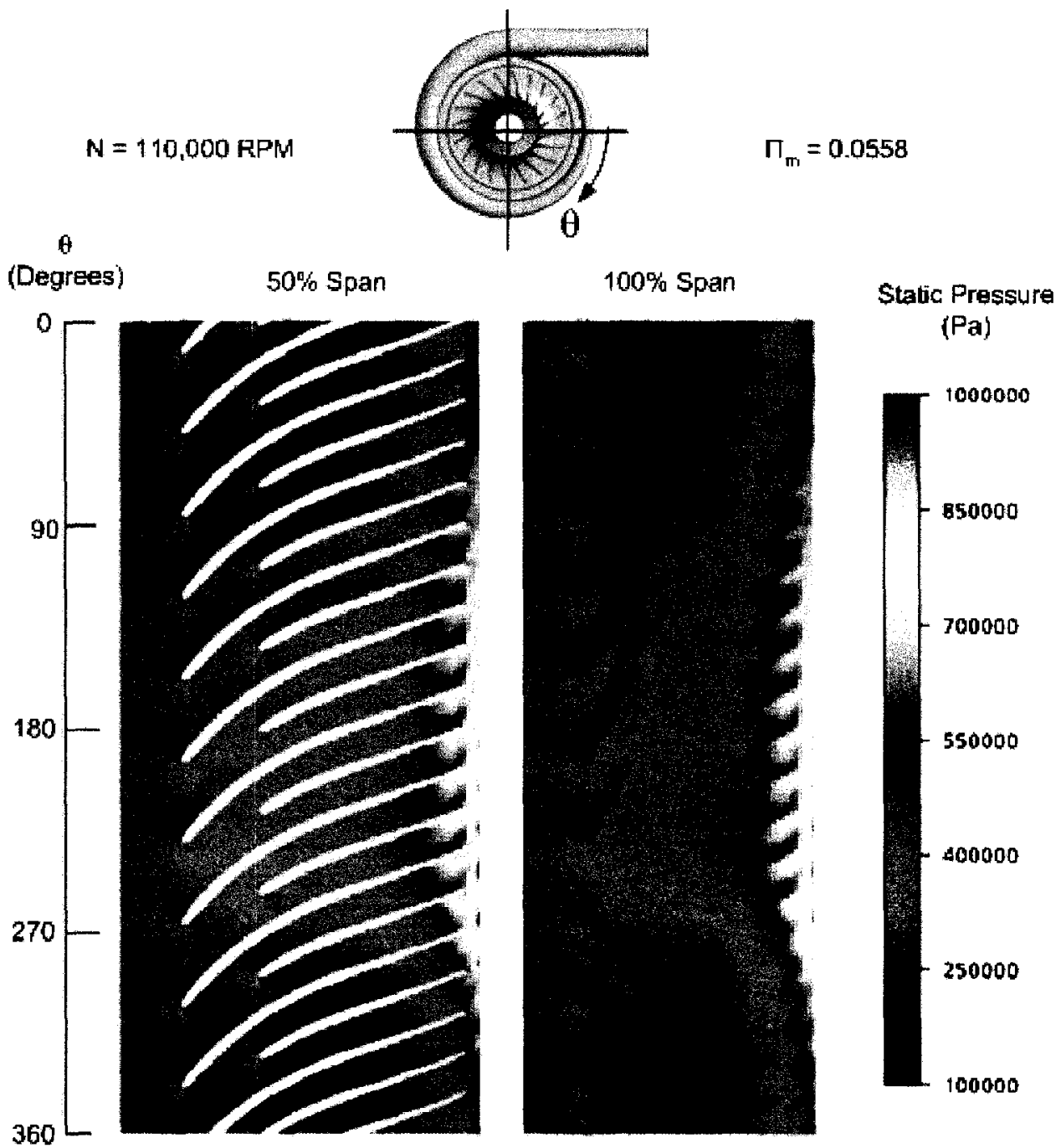

Figure 8.13: Contours of static pressure at multiple spans in the test rig impeller predicted by ANSYS CFX for the complete stage

Figure 8.13 shows the static pressure variation at constant span locations of $50 \%$ and $100 \%$. A circumferential variation is again seen at the leading and trailing edges on both planes. More interestingly the trends seen in the static pressure at the shroud $(100 \%$ span) are also evident within the vane passage of the rotor (50\% span). Similar results were obtained for the Turbonetics T04E-50 impeller predictions. This again suggests that if the CFD predictions are correct, experimental data taken from the shroud surface can give some insights into the flow within the vane passages of the impeller. As mentioned 
previously, this would require the measurement of instantaneous static pressures on the shroud surface using fast response pressure transducers, which are not currently available in the rotating test rig.

The experimental results for the Turbonetics T04E-50 impeller did not show such extreme circumferential variations. Although the circumferential non-uniformities were evident near the volute, there was little variation near the trailing edge or elsewhere within the impeller. The differences in the circumferential variations of the T04E-50 impeller and the new test rig impeller are thought to be in part due to the shorter vaneless diffuser length present in the new test rig impeller. It is therefore possible that significant effects upstream can be the result of downstream components. Thus, accurate aerodynamic predictions of centrifugal compressor designs cannot always be obtained using a model of the impeller alone.

Figure 8.14 shows the contours of absolute total pressure at mid-span of the test rig impeller. The cross-sections indicated as 1 and 2 are used to generate the exit plane total pressure and flow angle distributions discussed in the next section. They were selected since they represent regions of low and high total pressure at the impeller exit. The cross-sections range from $0^{\circ}$ to $36^{\circ}$ and $180^{\circ}$ to $216^{\circ}$ circumference respectively and represent the flow from one complete blade-splitter pair. As seen in Figure 8.14 the majority of the compression is occurring near the trailing edge and is expected based on the increased blade speed at larger radii. The regions of highest total pressure near the trailing edge occur at approximately $270^{\circ}$. This circumferential location corresponds to the region of the tongue and indicates its possible influence on the impeller flow. 


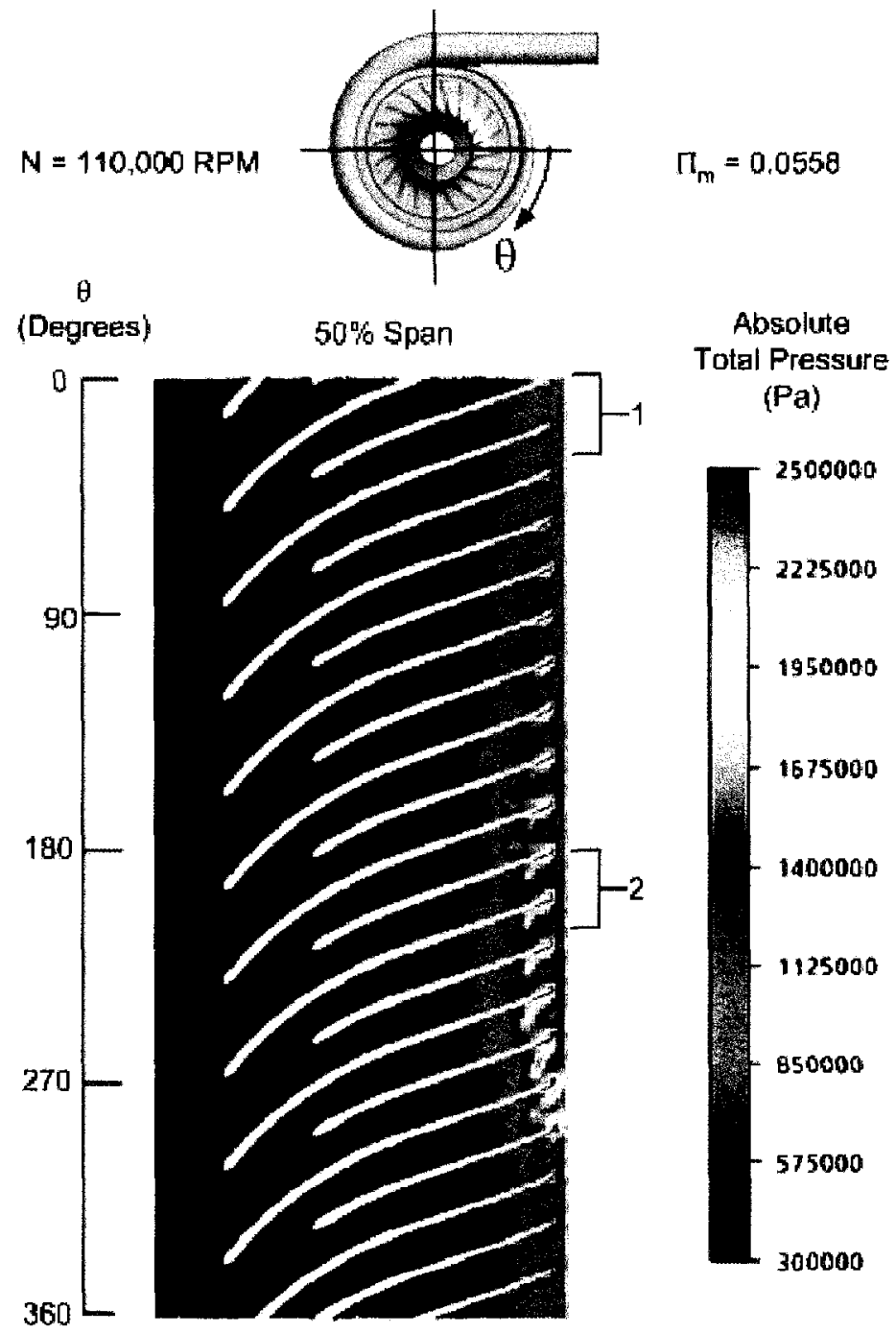

Figure 8.14: Contours of absolute total pressure at mid-span of the test rig impeller predicted by ANSYS CFX for the complete stage

\subsubsection{Exit Plane Total Pressure and Flow Angle Distributions}

The variations of absolute total pressure at the impeller exit plane from $0^{\circ}$ to $36^{\circ}$ and $180^{\circ}$ to $216^{\circ}$ are shown for three spanwise locations in Figures 8.15 and 8.16 respectively. The circumferential locations are indicated on Figure 8.14. The impeller exit plane is located downstream of the trailing edge at $100 \%$ of the trailing edge passage height. 
Figure 8.15 shows the circumferential region with the lower total pressure: region 1 on Figure 8.14. As seen, the total pressure varies by approximately $0.6 \mathrm{MPa}$ across the three spanwise locations. This variation corresponds to about $85 \%$ of the dynamic pressure mass-averaged over the data plane. Clearly, the spanwise variation of total pressure indicates that total pressure measurements made at a single spanwise location, as can be obtained on the rotating test rig, will not give an accurate measure of the average total pressure at the rotor outlet.

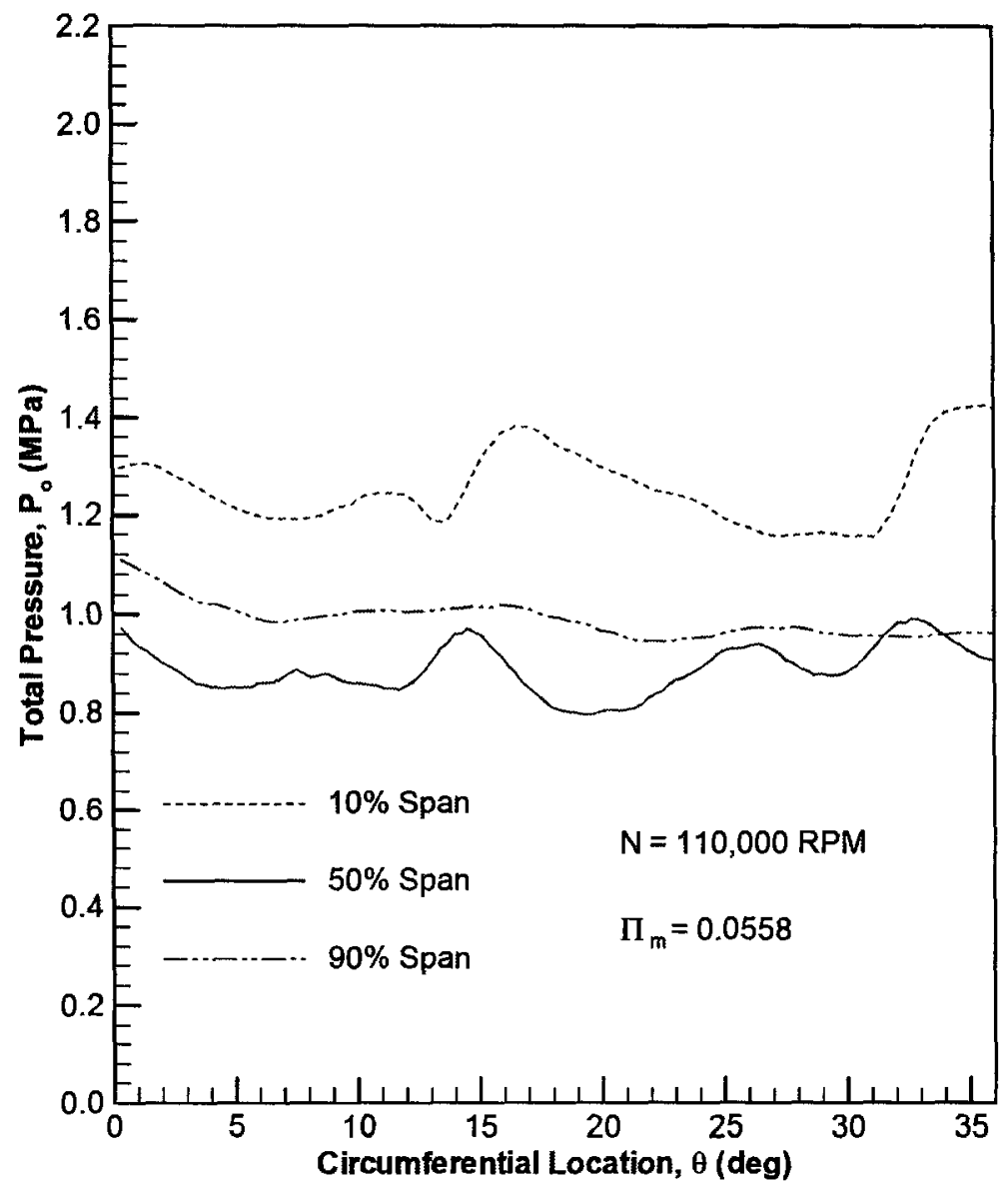

Figure 8.15: Variation of total pressure between $0^{\circ}$ and $36^{\circ}$ circumference at the exit plane of the test rig impeller predicted in the complete stage 


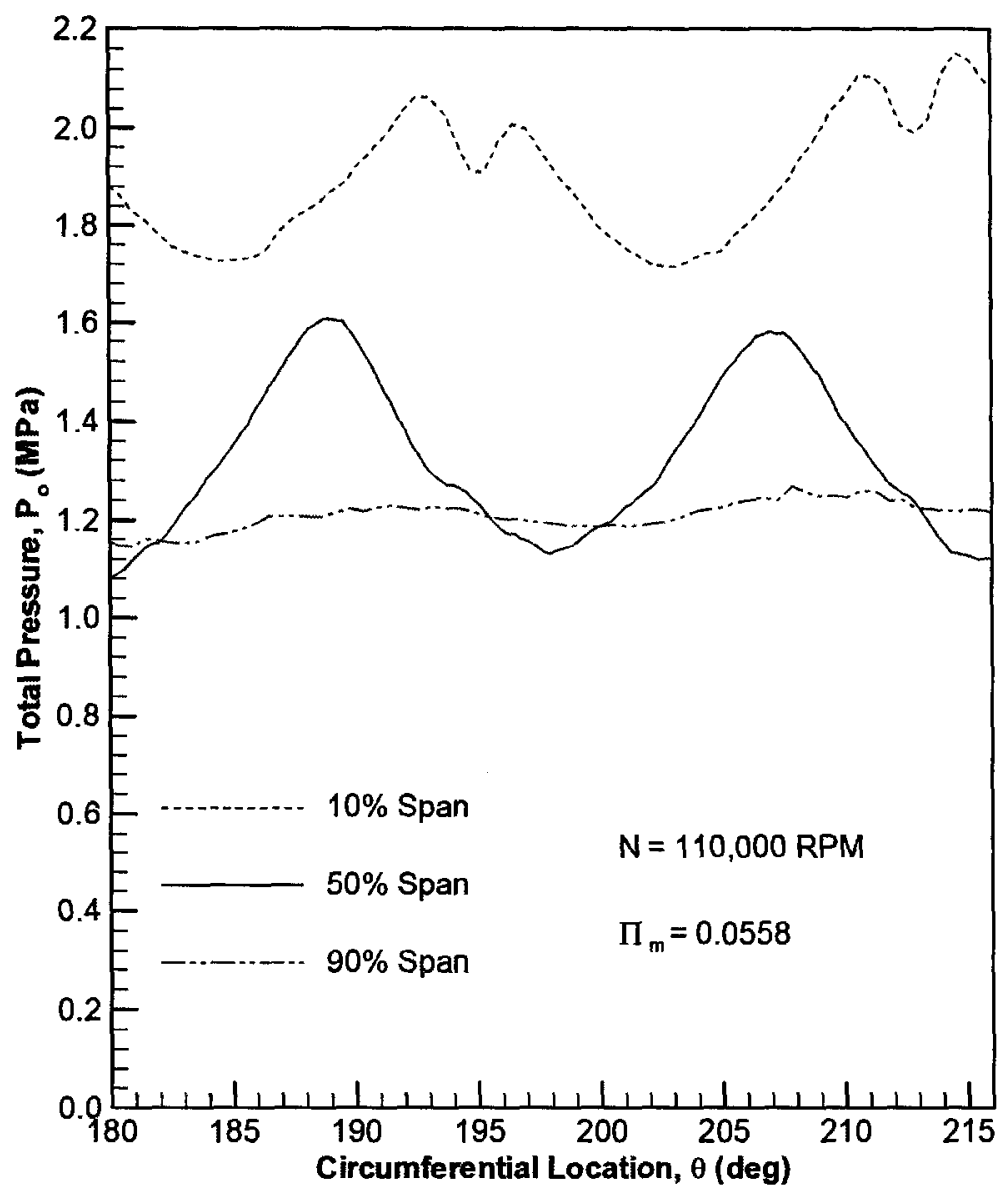

Figure 8.16: Variation of total pressure between $180^{\circ}$ and $216^{\circ}$ circumference at the exit plane of the test rig impeller predicted in the complete stage

Figure 8.16 shows the variation of total pressure at the exit plane of the impeller between $180^{\circ}$ and $216^{\circ}$; the higher total pressure region indicated as cross-section 2 on Figure 8.14. As seen, the low total pressure regions of the blade wakes are clearly defined at both $10 \%$ and $50 \%$ span. At $90 \%$ span in both circumferential regions the total pressure variation is nearly uniform. This is somewhat surprising since these calculations are downstream of the tip gap and the flow should be part of the tip leakage flow. The uniformity of the total pressure suggests that the tip leakage flow has not yet rolled up into the expected tip leakage vortex. The spanwise variation of total pressure 
between $180^{\circ}$ and $216^{\circ}$ is larger than that between $0^{\circ}$ and $36^{\circ}$ and further emphasizes the challenges involved in measuring the impeller exit total pressure in experimental testing.

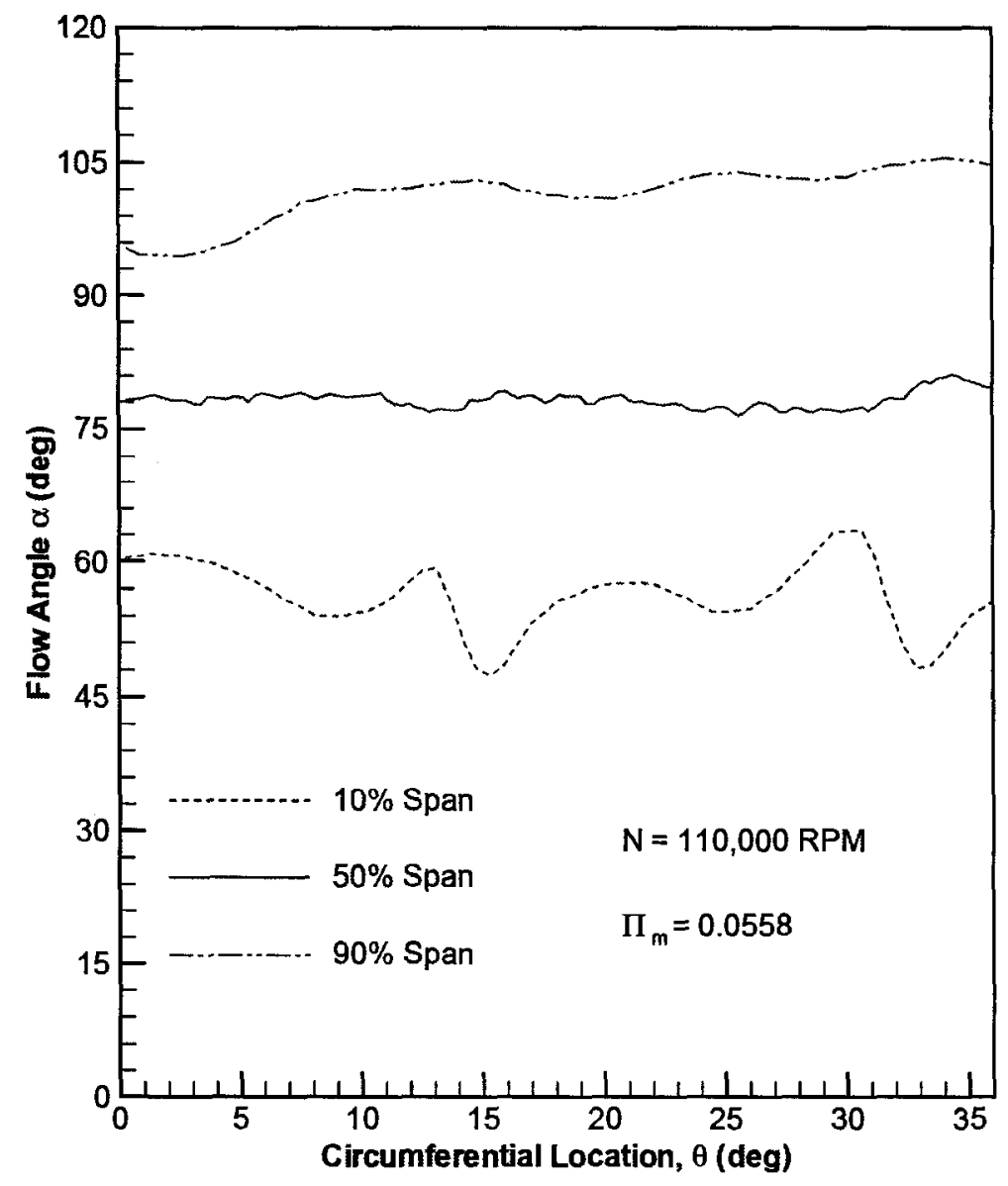

Figure 8.17: Variation of flow angle between $0^{\circ}$ and $36^{\circ}$ circumference at the exit plane of the test rig impeller predicted in the complete stage

Figures 8.17 and 8.18 show the flow angle variations at the three constant span locations from $0^{\circ}$ to $36^{\circ}$ and $180^{\circ}$ to $216^{\circ}$ respectively. The spanwise variation of flow angle is greater than $50^{\circ}$ and any flow angle measurements will clearly also be sensitive to the spanwise positioning of the measurement probes at the exit plane. 


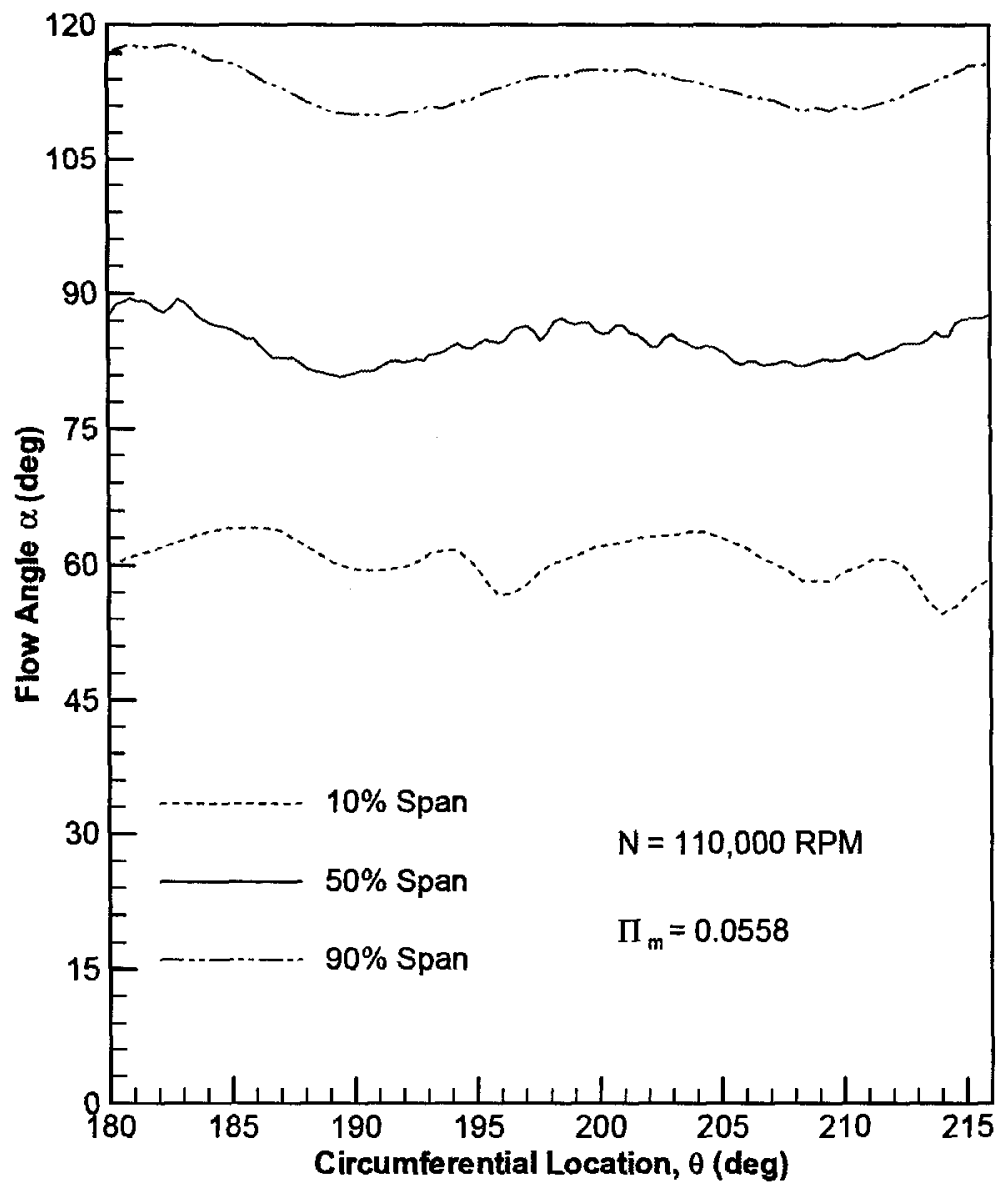

Figure 8.18 Variation of flow angle between $180^{\circ}$ and $216^{\circ}$ circumference at the exit plane of the test rig impeller predicted in the complete stage

\subsubsection{Blade Loadings}

The loading distributions for two representative blade sets are shown in Figures 8.19 and 8.20. The selected blades are indicated on Figure 8.11 and represent the extremes seen in the aerodynamic loading of all blades. Blade set A-B is located with the trailing edges close to the $0^{\circ}$ circumferential position and lies within a region of higher relative Mach numbers and lower relative total pressure. Blade set C-D is located with the trailing edges close to $180^{\circ}$ circumference and lies within a region of lower relative Mach numbers and higher relative total pressure. 

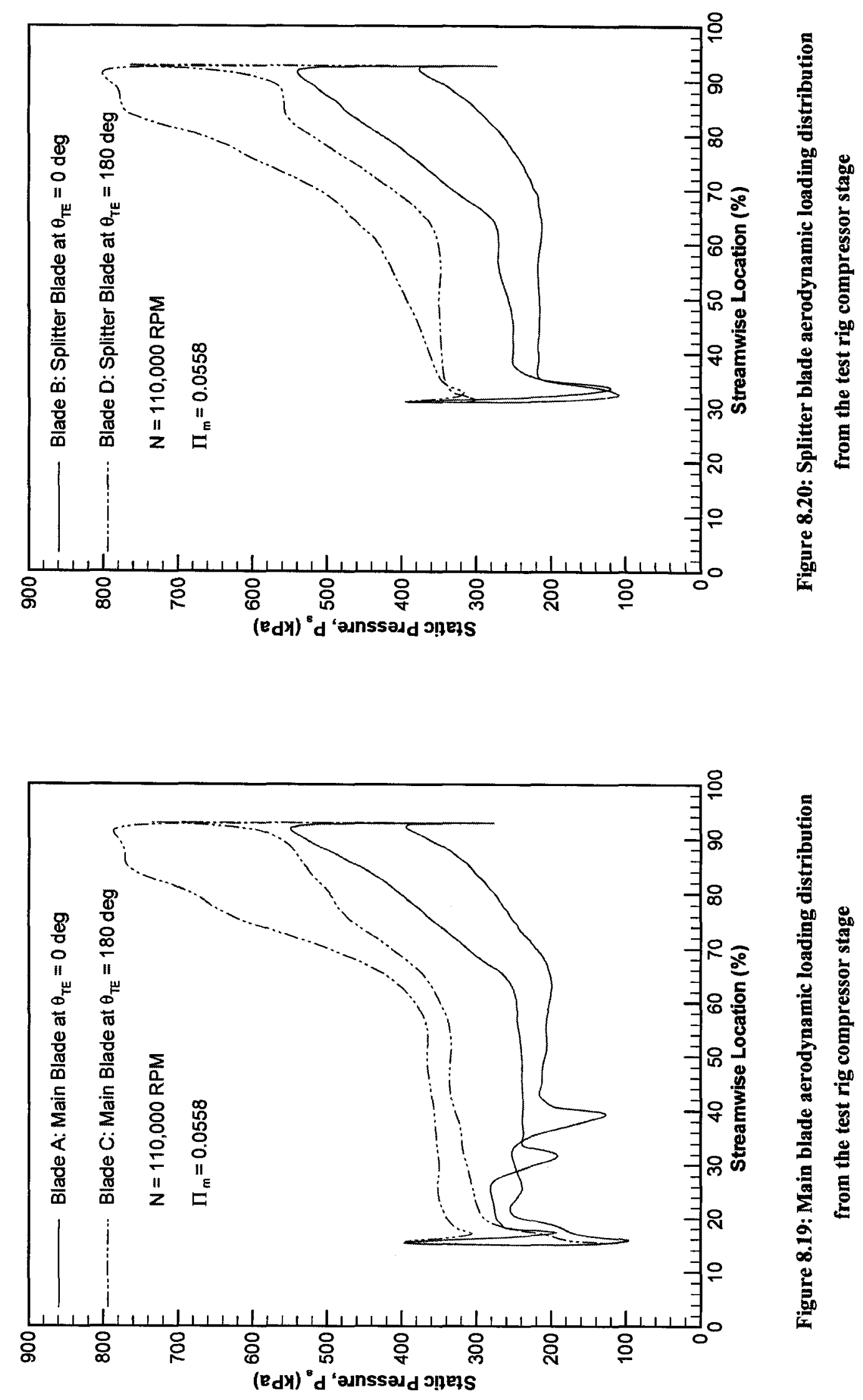
The majority of the blade force occurs after the $60 \%$ streamwise location for both the main blades (Fig. 8.19) and the splitter blades (Fig. 8.20). The effects of the nonuniformity of the volute are also seen in the loading distributions and extend to the leading edge. In particular, the static pressure is higher along blade set C-D because of the reduced Mach numbers in the leading edge region. In addition, the circumferential non-uniformity has resulted in local over speeds at the leading edge of blades A-B which have affected their respective loading distributions. The effect on the leading edge of the splitter blade B is sufficient to cause a region of reversed loading on the main blade A at the same streamwise position.

\subsection{Volute PERFormanCE AND ITS EFFECT ON THE IMPELLER}

\subsubsection{Performance Characteristics With and Without Volute}

The total pressure and isentropic efficiency characteristics of the impeller within the complete stage are shown in Figures 8.21 and 8.22 respectively. The impeller performance within the stage was obtained from the same computational runs as the complete stage; however the results were generated for an outlet plane at the trailing edge of the rotor instead of the volute outlet. The impeller performance within the complete stage is also compared to the overall stage and impeller only characteristics presented in Section 8.3.1. The indicated operating point is used for more detailed examination of the aerodynamic results for the volute, as discussed later.

As expected the total pressure rise and efficiency is lower for the complete stage than for the impeller alone or for the impeller within the stage. This is a result of the large total pressure losses through the volute particularly at the higher rotational speed. 


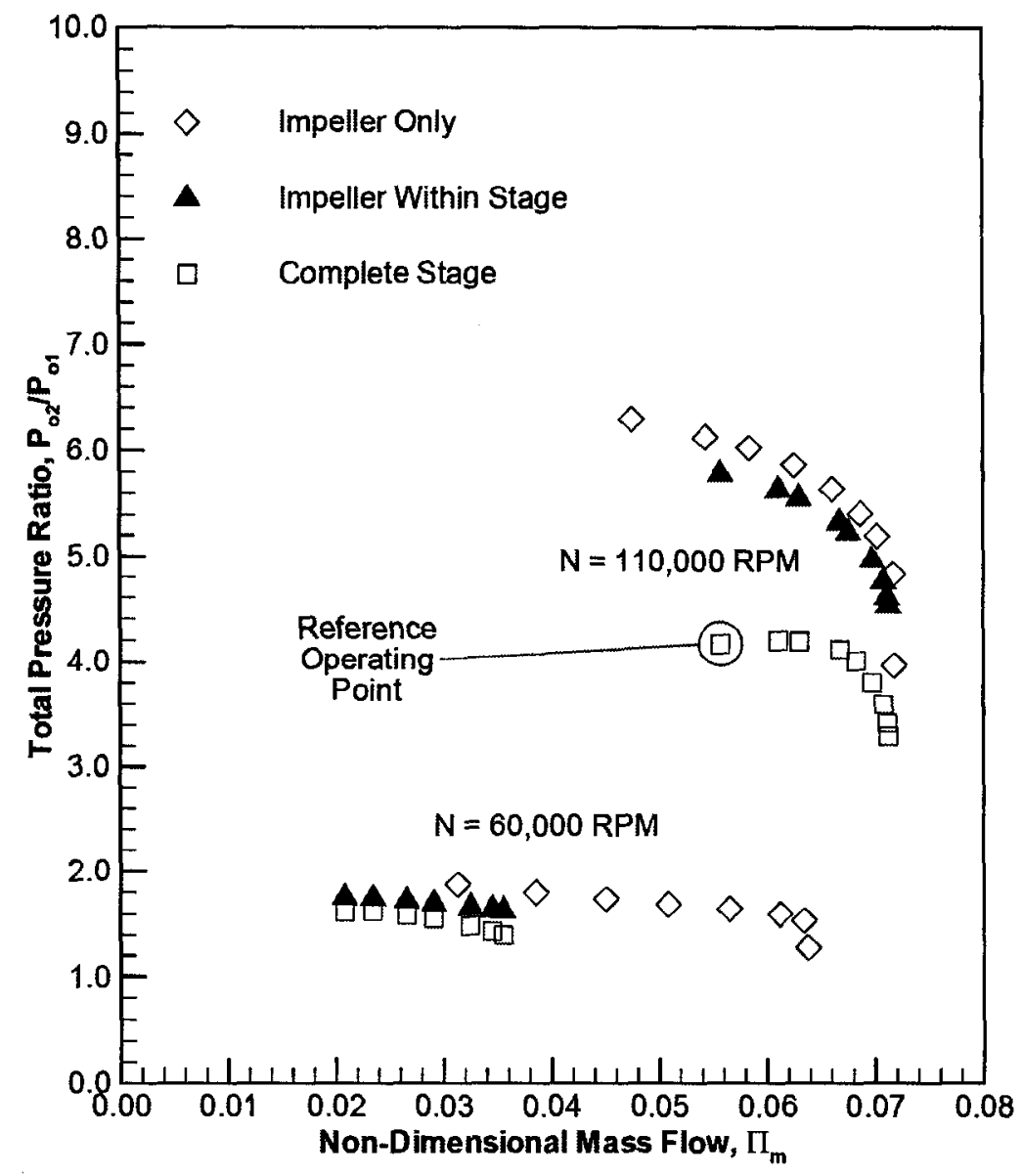

Figure 8.21: Total pressure ratio characteristic of the test rig impeller with and without the volute predicted by ANSYS CFX

The volute loss coefficient $(K)$ is defined as:

$$
K=\frac{P_{01}-P_{02}}{P_{01}-P_{S 1}}
$$

where the subscripts 1 and 2 denote the inlet and outlet of the volute respectively. The volute loss coefficient is predicted to be between approximately 0.5 and 0.6 for all unchoked operating points on the 110,000 RPM speed line. From Japikse and Baines (1997) the volute loss coefficient for well designed machines is typically between 0.2 and 
0.4. Clearly the volute is not appropriately designed for the given operating conditions. The poor performance of the volute is largely a result of the vaneless diffuser used in the test rig compressor. Although originally designed as a vaned diffuser, the diffuser was manufactured without vanes due to manufacturing constraints. Since the diffuser length remained unchanged the flow is not sufficiently diffused and the flow entering the volute has a much higher velocity and flow angle than originally designed for.

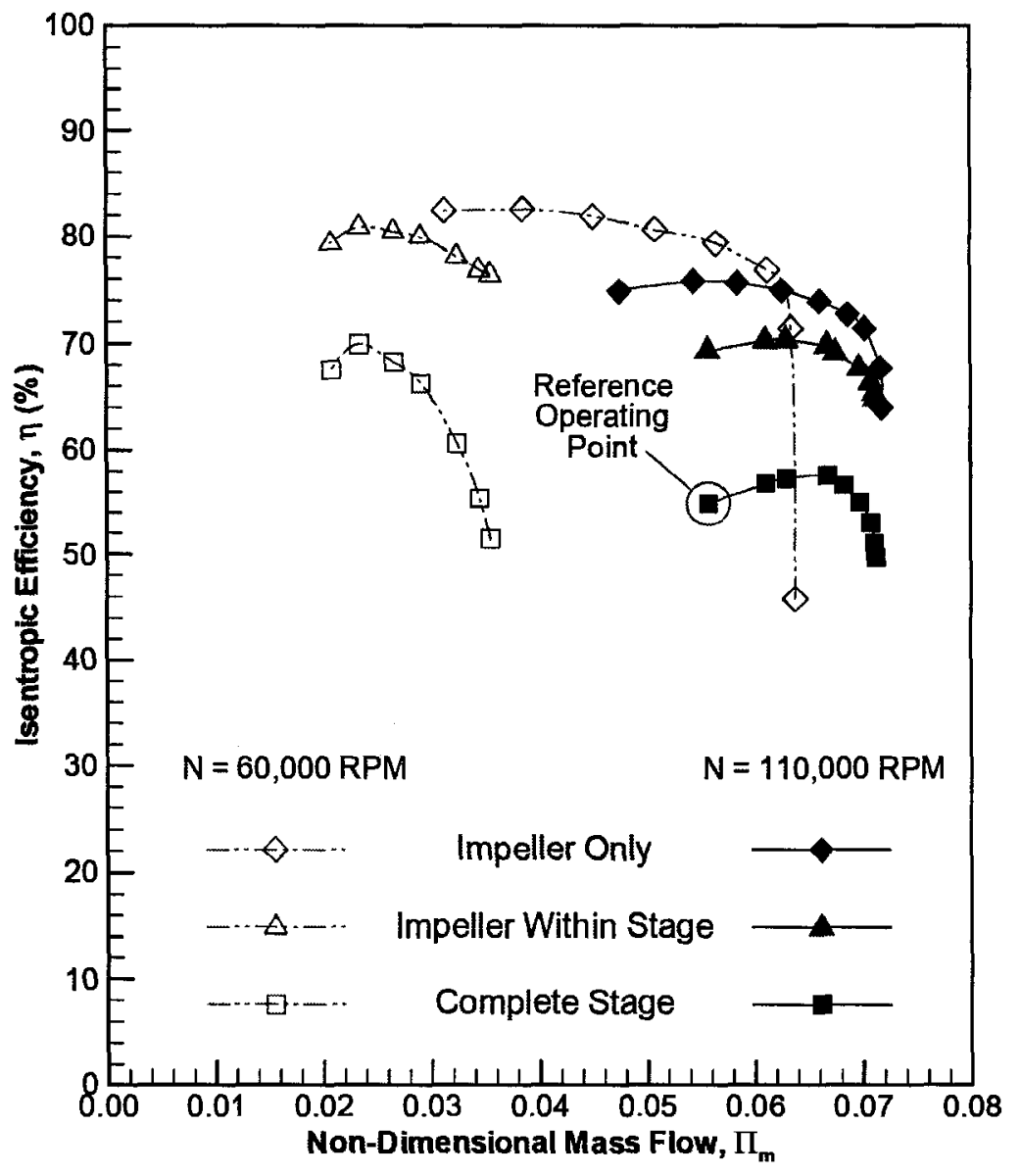

Figure 8.22: Efficiency characteristic of the test rig impeller with and without the volute predicted by ANSYS CFX

The volute is reducing the potential overall stage performance that could be achieved with a more appropriate design. In addition to the reduction of the stage total 
pressure rise and efficiency, the volute is also affecting the performance of the impeller within the stage. As mentioned on several occasions the presence of the volute has created circumferential non-uniformities within the diffuser and the impeller. It appears that some total pressure loss may have been incurred in the impeller due to the upstream effects of the volute. This is evident from Figures 8.21 and 8.22 because the resulting total pressure rise and efficiency of the impeller with the volute present is lower than the impeller alone. However, as seen from the experimental measurements of the T04E-50 compressor, a well designed diffuser of sufficient radial length could prevent circumferential non-uniformities of the volute from being propagated upstream to the impeller.

\subsubsection{Mach Number and Total Pressure Contours}

It has been shown that the presence of the volute within the compressor stage has reduced the performance of the impeller. It is believed that circumferential nonuniformities induced in the impeller by the volute are one cause of this performance reduction. To provide more insight into the cause of the circumferential non-uniformity more detailed predictions of the flow through the volute have also been obtained.

Figure 8.23 shows the Mach number contours through the volute domain on a plane that corresponds to $50 \%$ span at the impeller trailing edge. The operating point used is indicated on Figures 8.21 and 8.22. The flow enters the volute computational domain at approximately halfway through the diffuser passage. As seen in Figure 8.23, around the majority of the circumference the flow enters with circumferential variations due to the rotor blade wakes only. The locations of the wakes are fixed relative to the volute because the complete stage solutions were obtained using the Frozen-Rotor approximation, as discussed in Section 5.3. Between the circumferential positions of $135^{\circ}$ and $225^{\circ}$ there is a region of high Mach numbers that extend through the diffuser passage and into the volute. 


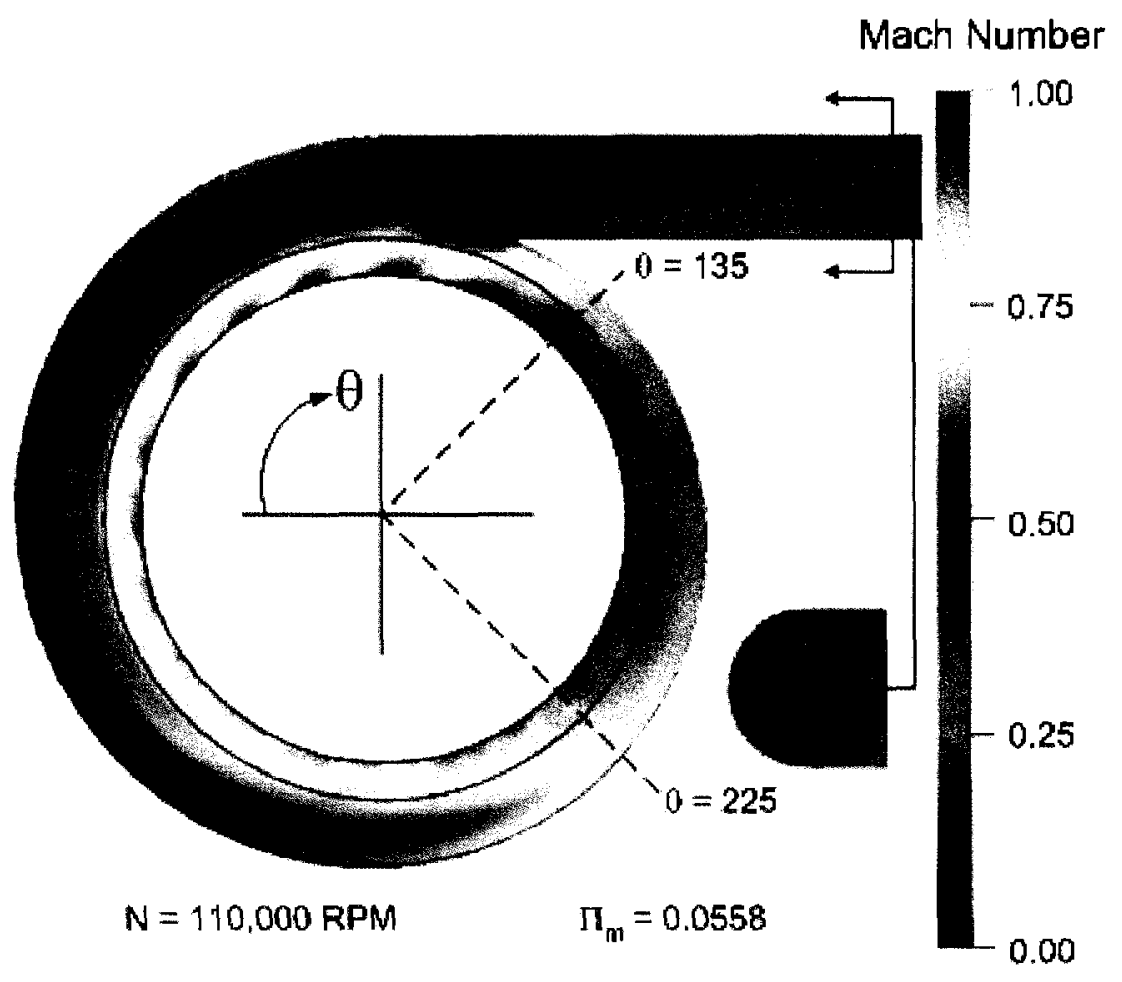

Figure 8.23: Contours of Mach number within the volute domain

Figure 8.24 shows the mass flow rate distribution around the circumference of the volute inlet. Each symbol represents the mass flow rate through a $10^{\circ}$ section of the inlet divided by the section area. As seen the mass flow rate per unit area is highest between the $135^{\circ}$ and $225^{\circ}$ circumferential positions. Thus the high Mach numbers (Fig. 8.23) in this circumferential range are the result of increased mass flow rate entering the volute computational domain in this region. The circumferential non-uniformities are therefore a result of the volute's inability to swallow the flow uniformly. Figure 8.24 also shows a sudden decrease in mass flow rate in the tongue region (between $90^{\circ}$ and $135^{\circ}$ ) and further illustrates the tongues influence on the flow. 


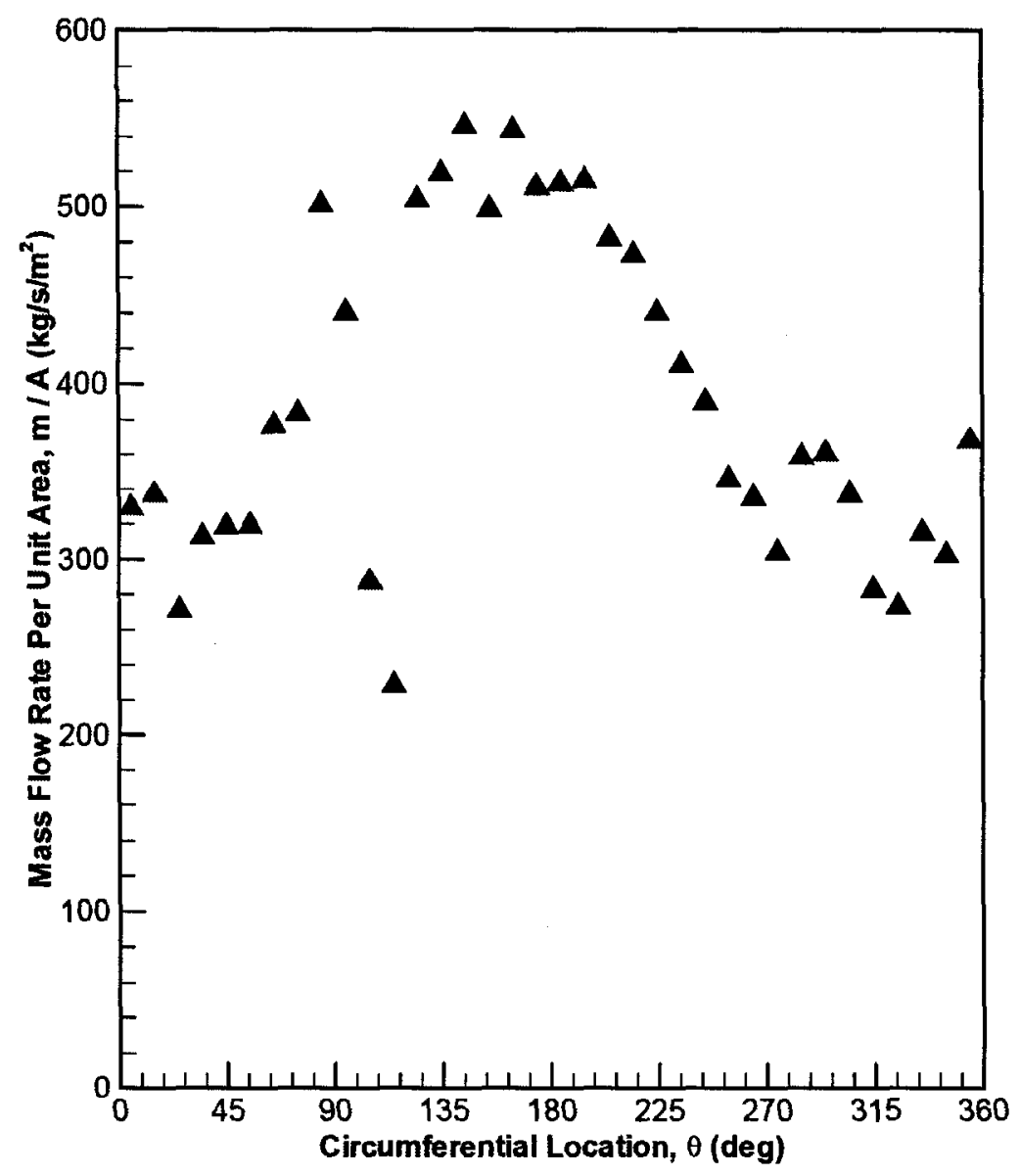

Figure 8.24: Mass flow rate distribution at the volute inlet

Despite the non-uniformity, the volute delivers the flow to the discharge pipe with a relatively uniform Mach number. The cross-section inset in Figure 8.23 is re-plotted in Figure 8.25 with a reduced Mach number scale. The flow near the outlet has a massaveraged Mach number of approximately 0.4 distributed relatively uniformly around the area. 


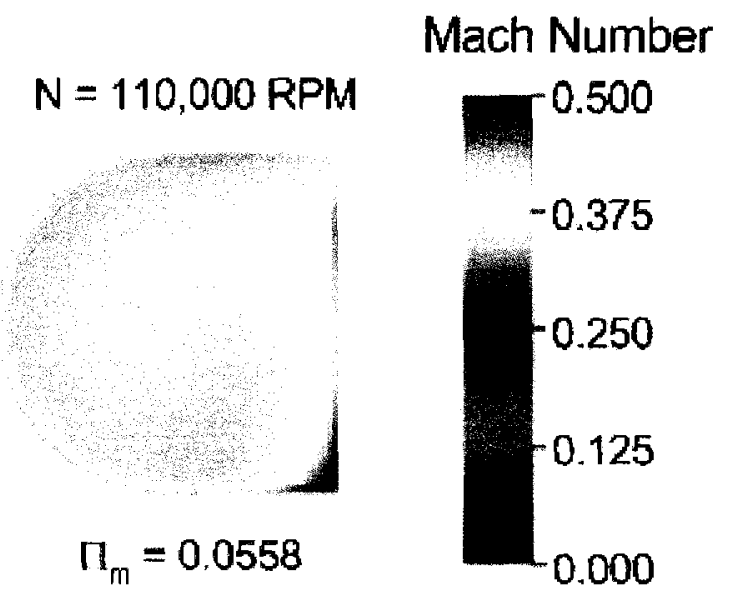

Figure 8.25: Mach number distribution near the volute exit

Figure 8.26 shows the total pressure contours at the same axial plane as the results in Figure 8.23. As seen there are variations in the total pressure around the circumference of the volute. In particular there is a region of lower total pressure from the $0^{\circ}$ to $90^{\circ}$ circumferential positions. The reduced total pressure seems to originate from within the impeller, but this may be induced an upstream effect of the volute. Again the cross-section inset in Figure 8.26 is re-plotted with a reduced scale in Figure 8.27. The total pressure variation across the outlet area is approximately $0.15 \mathrm{MPa}$, which is $12 \%$ of the mass-averaged total pressure and $120 \%$ of the mass-averaged local dynamic pressure. 


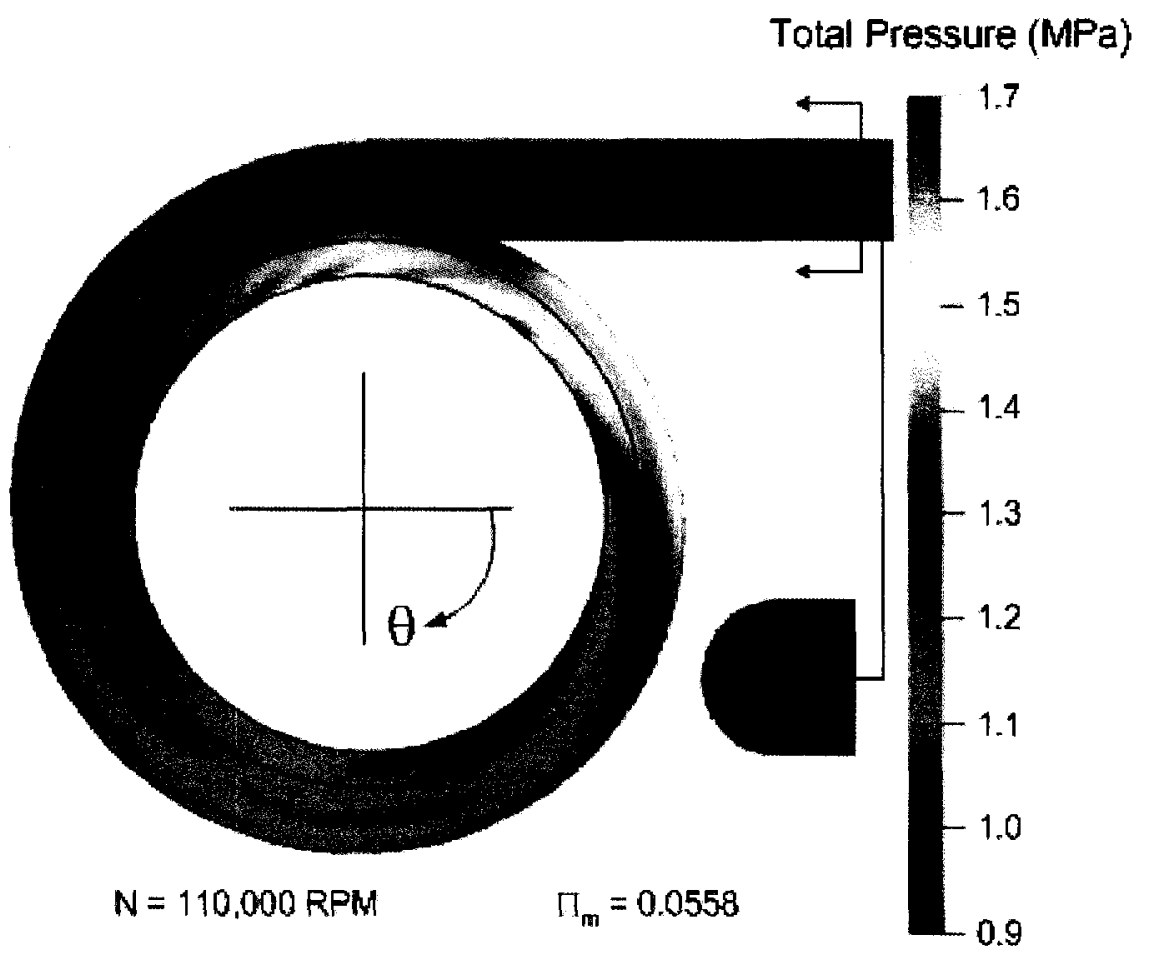

Figure 8.26: Contours of total pressure within the volute domain

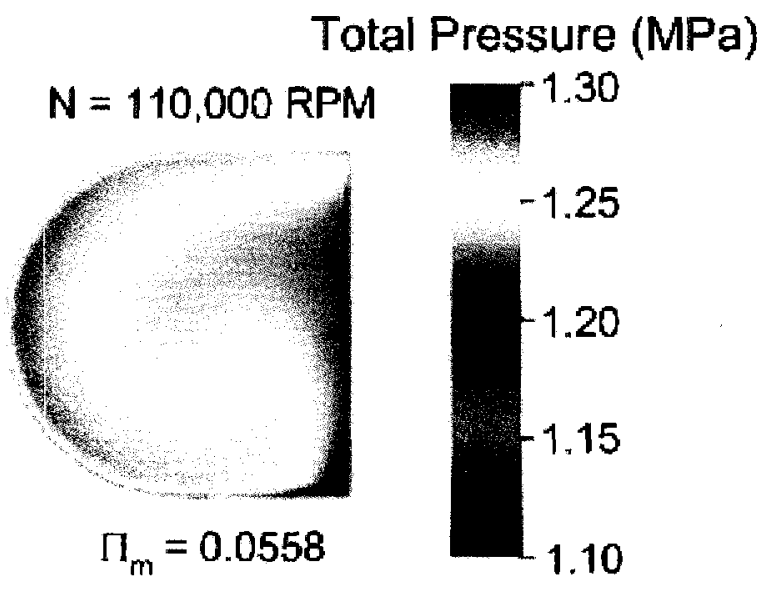

Figure 8.27: Total pressure distribution near the volute exit 


\subsection{CONCLUSIONS AND RECOMMENDATIONS FOR FUTURE WORK}

\subsection{Conclusions}

The design of the new rotating test rig centrifugal compressor impeller has been presented. The impeller was designed to eliminate manufacturing problems present in a previous impeller designed for the Raven Zero-Emission Gas Turbine compressor. The new test rig impeller was successfully manufactured by Deloro-Stellite. Preliminary CFD predictions of the test rig impeller, performed using CFX-BladeGen, have been presented. In addition, the test rig impeller was used to further investigate the influence of working fluid on the impeller design geometry. This process also used CFXBladeGen. It was found that simple geometric modifications could partially counteract the influence of working fluid on the impeller performance. However, it was found that the geometric modifications could be used to adjust the aerodynamic performance at only one operating point on a given constant speed operating line.

More detailed aerodynamic predictions of the test rig impeller were then made using ANSYS CFX. These computations were applied to both the impeller only and the complete stage. It was found from these predictions that significant losses in the test rig compressor performance were to be expected as the result of the geometries of the vaneless diffuser and the volute. In addition, the effects of the volute seemed to extend into the impeller, which suggests that compressor performance cannot be predicted reliably using a model of only the impeller unless the diffuser extends significantly in the radial direction. The detailed aerodynamic predictions also showed significant spanwise and circumferential variations of flow velocity, flow angularity and total pressure downstream of the impeller trailing edge. This indicates that detailed circumferential and 
spanwise measurements are needed to obtain experimental data that can be used to accurately determine the impeller only overall performance.

Due to time constraints the collection of experimental data for the test rig impeller was not possible. Instead, experimental data were collected and presented for an existing Turbonetics T04E-50 compressor. Modifications were made to the rotating test rig to allow the total pressure and flow angle to be measured in the diffuser passage near the impeller exit. The measurements were taken at approximately midspan and were compared to detailed aerodynamic performance predictions of the T04E-50 impeller along the same spanwise location. Reasonable agreement was found between the experimental data and the CFD predicted values at midspan for the range of operating points that could be examined. However, the aerodynamic predictions also showed significant spanwise variations in the flow near the impeller exit. Similar to the test rig impeller, the aerodynamic predictions of the T04E-50 impeller indicate that measurements made at midspan near the impeller exit will not be representative of the mass-averaged impeller outlet flow. The test rig modifications also included the addition of shroud surface static pressures. Again, these measurements were compared to the computations and reasonable agreement was found.

\subsection{RECOMMENDATIONS FOR FUTURE WORK}

The next step following from the present work is to obtain experimental performance results for the re-designed test rig compressor for comparison to the CFD predictions presented here. Before the test rig impeller is installed in the rotating test rig the impeller will need to be dynamically balanced. In addition, the manufacture of the compressor casing still needs to be completed. The CFD predictions of the test rig impeller, with zero tip clearance modelled, were performed using a reduced passage height. This was the case for the calculations using CFX-BladeGen+ and ANSYS CFX. To provide a more appropriate comparison to the predictions of the test rig impeller with 
tip clearance modelled, the calculations with zero tip clearance should be performed using the full, actual passage height. It would also be worthwhile to perform CFD predictions for the new test rig compressor using an extended radial diffuser length. By extending the radial length of the diffuser it may be possible to reduce the circumferential variations near the impeller outlet and reduce the uncertainty in the rotatable total pressure probe measurements. The volute would also need to be re-designed and a new compressor casing would have to be manufactured such that the experimental data and the CFD predictions are obtained using the same geometry.

Time permitting it would have been desirable to obtain more experimental data using the rotatable total pressure probes. By collecting more data using the same measurement methods it would be possible to provide an estimate of the repeatability of the measurements. In addition, the productivity and quality of the rotatable total pressure probe measurements could be improved by automating their rotation. This would allow the probe angles to be altered during a given run, which should significantly reduce the time requirements for measuring a single operating point. In addition, the uncertainty in matching operating points from run to run would be eliminated.

The effect of working fluid on the aerodynamic performance of a compressor is still not fully understood. One possible approach for investigating this issue further would be to perform an analysis of the impeller performance with working fluids with similar isentropic exponents and different gas constants.

Lastly, the accuracy of the ANSYS CFX predictions of the test rig compressor might be improved in a couple of ways:

- Obtain time accurate solutions for all computational cases.

- Specify all walls of the diffuser as separate surfaces. This will allow the portion of the hub wall which extends into the diffuser from the impeller domain to be 
specified as a stationary wall and should result in more physically realistic predictions of the flow in the diffuser.

Further attempts should also be made to obtain converged solutions at operating points with lower mass flow rates. This would provide more operating points for comparison to experimental data. Solutions for a wider range of mass flow rates may be possible using time accurate calculations. 


\section{REFERENCES}

ANSYS CFX v10.0 User's Manual (2005), ANSYS Europe Ltd. (Supplied with Software).

Aungier, R.H. (2000), Centrifugal Compressors: A Strategy for Aerodynamic Design and Analysis, ASME Press, New York.

CFX BladeGen+ v. 4.0.018 User's Manual (2000), AEA Technology (Supplied with Software)

Charest, M. (2003), "Compressor: Impeller Preliminary Design Using a Refined Slip Factor Approach", DR 497-02E/190/003A, MECH 4907 Gas Turbine Project, Carleton University, Ottawa, Canada.

Colebrook, C.F. (1939), "Turbulent Flow in Pipes, With Particular Reference to the Transition Region Between the Smooth and Rough Pipe Laws", J. Inst. Civ. Eng., 11, pp. 133-156 (As referenced by Haaland, 1983).

Corradetti, A. and Desideri, U. (2004), "Analysis of Gas-Steam Combined Cycles With Natural Gas Reforming and $\mathrm{CO}_{2}$ Capture", Proceedings of ASME Turbo Expo 2004, GT2004-54091, Vienna, Austria.

Del Greco, A.S., Biagi, F.R., Sassanelli, G. and Michelassi, V. (2007), “A New Slip Factor Correlation for Centrifugal Impellers in a Wide Range of Flow Coefficients and Peripheral Mach Numbers", Proceedings of ASME Turbo Expo 2007, GT2007-27199, Montreal, Canada. 
De Ruyck, J. (1992), "Efficient $\mathrm{CO}_{2}$ Capture Through a Combined Steam and $\mathrm{CO}_{2}$ Gas Turbine Cycle", Energy Conversion and Management, Vol. 33, No.5-8, pp. 397403.

Dixon, S.L (1998), Fluid Mechanics and Thermodynamics of Turbomachinery, Butterworth-Heinemann, Boston.

Haaland, S.E. (1983), "Simple and Explicit Formulas for the Friction Factor in Turbulent Pipe Flow", ASME J. of Fluids Eng., 105, pp. 89-90.

Harman, R.T.C. (1981), Gas Turbine Engineering: Applications, Cycles, and Characteristics, MacMillan Press, New York.

Hunt, J. (2004), "Investment Casting: Test Rig Compressor Manufacturing Issues", DR 4907-03GT/260/003, MECH 4907 Gas Turbine Project, Carleton University, Ottawa, Canada.

Irvine, T.F. and Liley, P.E. (1984), Steam and Gas Tables with Computer Equations, Academic Press, Orlando.

Jackson, J.B., Neto, A.C., Whellens, M.W., and Audus, H. (2000), "Gas Turbine Performance Using Carbon Dioxide as Working Fluid in Closed-Cycle Operation", Proceedings of ASME Turbo Expo 2000, 2000-GT-153, Munich, Germany.

Japikse, D. and Baines, N.C. (1997), Introduction to Turbomachinery, Concepts ETI Inc., Whiter River Junction and Oxford University Press, Oxford. 
Jeffries, M. (1994), The Commissioning and Enhancement of a Blow Down Wind Tunnel, M.Eng. Thesis, Dept. of Mech. and Aero. Eng., Carleton University, Ottawa, Canada.

Jeffries, M. (2001), Initial Investigations of Transonic Turbine Aerodynamics Using the Carleton University High-Speed Wind Tunnel, Ph.D. Thesis, Dept. of Mech. and Aero. Eng., Carleton University, Ottawa, Canada.

Jericach, H. and Gottlich, E. (2002), “Conceptual Design for an Industrial Prototype Graz Cycle Power Plant", Proceedings of ASME Turbo Expo 2002, 2002-GT-30118, Amsterdam, The Netherlands.

Jericha, H., Sanz, W. and Gottlich, E. (2006), "Design Concept for Large Output Graz Cycle Gas Turbines", Proceedings of ASME Turbo Expo 2006, GT2006-90032, Barcelona, Spain.

Kang, S. (2005), "Numerical Investigation of a High Speed Centrifugal Compressor Impeller", Proceedings of ASME Turbo Expo 2005, GT2005-68092, Reno-Tahoe, USA.

Krain, H., Hoffman, B., Rohne, K.H., Eisenlohr, G. and Richter, F.A. (2007), "Improved High Pressure Ratio Centrifugal Compressor", Proceedings of ASME Turbo Expo 2007, GT2007-27100, Montreal, Canada.

Marconcini, M., Rubechini, F. and Arnone, A. (2006), "Numerical Investigation of a Transonic Centrifugal Compressor", Proceedings of ASME Turbo Expo 2006, GT2006-90098, Barcelona, Spain. 
Mathieu, P., Dechamps, P., and Distelmans, M. (1994), "Concepts and Applications of $\mathrm{CO}_{2}$ Gas Turbines", Proceedings of Power-Gen Europe 1994, Cologne, Germany.

Mathieu, P., and De Ruyck, J. (1993), "The $\mathrm{CO}_{2}$ Gas Turbine Option for Recovery of $\mathrm{CO}_{2}$ from CC and IGCC Plants", ASME Cogen-Turbo Power 1993, IGTI-Vol. 8, pp. 77-83.

Moffat, R.J., (1985), "Using Uncertainty Analysis in the Planning of an Experiment", ASME J of Fluids Eng, 107, pp. 173-182.

Performance Test Code on Compressors and Exhausters, ASME PTC 10-1997.

Qiu, X., Mallikarachchi, C. and Anderson, M. (2007), “A New Slip Factor Model for Axial and Radial Impellers", Proceedings of ASME Turbo Expo 2007, Montreal, Canada.

Roberts, S.K (2001), Effects of Fluid Properties on the Aerodynamic Performance of Turbomachinery for Semi-Closed Cycle Gas Turbine Engines Using $\mathrm{O}_{2} / \mathrm{CO}_{2}$ Combustion, M.A.Sc. Thesis, Dept. of Mech. and Aero. Eng., Carleton University, Ottawa, Canada.

Rodgers, C. (2005), "Flow Ranges of 8.0:1 Pressure Ratio Centrifugal Compressors for Aviation Applications", Proceedings of ASME Turbo Expo 2005, GT2005-68041, Reno-Tahoe, USA.

Saravanamuttoo, H.I.H., Cohen, H. and Rogers, G.F.C. (2001), Gas Turbine Theory, Prentice-Hall, Toronto. 
Sjolander, S.A. (2008), MECH 5401: Turbomachinery, Dept. of Mech. and Aero. Eng., Carleton University, Supplementary Course Notes.

Strub, R.A., Bonciani, L., Borer, C.J., Casey, M.V., Cole, S.L., Cook, B.B., Kotzur, J., Simon, H., and Strite, M.A. (1987), "Influence of the Reynolds Number on the Performance of Centrifugal Compressors", ASME Journal of Turbomachinery, 109, pp. 541-544.

Shepherd, D.G. (1956), Principles of Turbomachinery, MacMillan Company, Toronto.

Ulizar, I. and Pilidis, P. (1999), "Handling of a Semiclosed Cycle Gas Turbine With a Carbon Dioxide-Argon Working Fluid", Proceedings of the International Gas Turbine \& Aeroengine Congress and Exhibition, 99-GT-374, Indianapolis, Indiana.

Urner, G. (1997), "Pressure Loss of Orifice Plates According to ISO 5167-1", Flow Measurement and Instrumentation, 8, pp 39-41.

Wang, B., Jin, H., Han, W. and Zheng, D. (2004), "IGCC System with Integration of $\mathrm{CO}_{2}$ Recovery and the Cryogenic Energy in Air Separation Unit", Proceedings of ASME Turbo Expo 2004, GT2004-53723, Vienna, Austria.

White, F.M. (1994), Fluid Mechanics, McGraw-Hill Inc., Toronto.

Wiesner, F.J. (1967), “A Review of Slip Factors for Centrifugal Impellers”, ASME J. of Engineering for Power, 89, pp. 558-572.

Wilson, D.G. and Korakianitis, T. (1998), The Design of High-Efficiency Turbomachinery and Gas Turbines, Prentice Hall, Upper Saddle River, NJ. 


\section{APPENDIX: CURVE FIT EQUATIONS OF FLUID PROPERTIES}

The following are the curve fit equations taken from Irvine (1984) used to determine the fluid properties of dry air and water vapour.

DRY AIR

$$
\begin{aligned}
& c_{P}(T)=\sum_{n=0}^{n=4} a_{n} T^{n} \quad \frac{\mathrm{kJ}}{\mathrm{kgK}} \\
& a_{0}=1.03409 \\
& a_{1}=-284.887 \times 10^{-6} \\
& a_{2}=781.6818 \times 10^{-9} \\
& a_{3}=-497.0786 \times 10^{-12} \\
& a_{4}=107.7024 \times 10^{-15} \\
& \gamma=\frac{c_{p}}{c_{p}-R} \\
& R=0.287040 \frac{\mathrm{kJ}}{\mathrm{kgK}}
\end{aligned}
$$

\section{WATER VAPOUR}

$$
\begin{aligned}
& c_{P}(T)=237.6 \times 10^{-9} T^{2}-97.9 \times 10^{-6} T+1.847 \quad \frac{\mathrm{kJ}}{\mathrm{kgK}} \\
& \gamma=\frac{c_{p}}{c_{p}-R}, \quad R=0.46189 \frac{\mathrm{kJ}}{\mathrm{kgK}}
\end{aligned}
$$




$$
\begin{array}{lll}
P_{\text {sat }}=e^{\left(\sum_{n=0}^{n=8} a_{n} T^{n}\right)}-\left(\frac{b}{T-c}\right) \quad \mathrm{kPa} & \\
a_{0}=10.4592 & a_{1}=-4.04897 \times 10^{-3} & a_{2}=-4.1752 \times 10^{-5} \\
a_{3}=3.6851 \times 10^{-7} & a_{4}=-1.0152 \times 10^{-9} & a_{5}=8.6531 \times 10^{-13} \\
a_{6}=9.03668 \times 10^{-16} & a_{7}=-1.9969 \times 10^{-18} & a_{8}=7.79287 \times 10^{-22} \\
a_{9}=1.91482 \times 10^{-25} & b=3968.06 & c=39.5735
\end{array}
$$

